MODE I FRACTURE OF EIGHT-HARNESS-SATIN CARBON CLOTH WEAVES FOR CO-CURED AND POST-BONDED LAMINATES

\author{
A Thesis \\ presented to \\ the Faculty of California Polytechnic State University, \\ San Luis Obispo
}

\author{
In Partial Fulfillment \\ of the Requirements for the Degree \\ Master of Science in Mechanical Engineering
}

by

Joshua Eli James Smith

December, 2013 
(C)-2013

Josh Smith

ALL RIGHTS RESERVED 
COMMITTEE MEMBERSHIP

TITLE:

AUTHOR:

DATE SUBMITTED:

COMMITTEE CHAIR:

COMMITTEE MEMBER:

COMMITTEE MEMBER:
Delaminations of Eight-Harness-Satin Carbon Cloth Weaves for Co-Cured and Post-Bonded Laminates

Joshua Eli James Smith

December, 2013

Dr. Joseph Mello, PhD

Mechanical Engineering Department

Dr. John Chen, PhD

Mechanical Engineering Department

Dr. Eric Kasper, $\mathrm{PhD}$

Civil Engineering Department 


\begin{abstract}
Delaminations of Eight-Harness-Satin Carbon Cloth Weaves for

Co-Cured and Post-Bonded Laminates

Joshua Eli James Smith
\end{abstract}

Mode I interlaminar fracture of 3k 8-Harness-Satin Carbon cloth, with identical fill and weft yarns, pre-impregnated with Newport 307 resin was investigated through the DCB test (ASTM D5528 [6]). Crack propagations along both the fill and weft yarns were considered for both post-bonded (co-bonded) and co-cured laminates. A patent-pending delamination insertion method was compared to the standard Teflon ${ }^{\circledR}$ film option to assess its applicability to mode I fracture testing. The Modified Beam Theory, Compliance Calibration method, and Modified Compliance Calibration method were used for comparative purposes for these investigations and to evaluate the validity of the proposed Equivalent Stiffness (EQS) method. Crack propagation, in all specimens, proceeded in a run-arrest manner for both delamination directions. Energy dissipation in the form of transverse yarn debonding, matrix deformation, and out of plane crack growth was witnessed for specimens with delaminations along weft yarns. A complete comparison between post-bonded and co-cured laminates was not achieved. The patent pending delamination insertion method was found to cause fewer instances of non-linear crack initiation behavior than the Teflon ${ }^{\circledR}$ insert and, when non-linear behavior did occur, it was less prevalent. The EQS method was found to achieve fracture toughness values within $5 \%$ of the other three data reduction methods for $63 \%$ of the propagation values and achieved conservative values for over $33 \%$ of the propagations. Suggestions for future studies aimed at completing the comparisons above are provided in Chapter 5. 


\section{ACKNOWLEDGMENTS}

I gratefully acknowledge the help and guidance of Dr. Joe Mello and Dr. John Chen throughout this research endeavor. Their willingness to provide assistance whenever I called is evidence of their devotion to teaching and the promotion of higher learning. I would also like to recognize Dr. Eric Kasper for serving on my thesis committee.

Many students at Cal Poly are very much in my debt for their assistance in this project. All of the students in Dr. Mello's Spring 2013 undergraduate composites class who layed up samples for my testing deserve special recognition, especially Corinne Warnock, Bobby Dodge, Nathan Cheadle, William Hilgenberg, Samantha Weiner, and Matt Baker. I would also like to extend a special recognition to Joey Marino for his constant assistance in the Double Cantilever Beam testing and his unceasing willingness to extend a helping hand.

Finally, special thanks to my brother, Evan, and my parents, Tim and Peggy for their unceasing love and support. I called my brother several times with requests for figures that I would like made or models that I would like him to draw. He never said no and was always happy to help. My parents were willing to drive 6.5 hours to endure four days of absolute misery helping their son. They payed for their own hotel, payed for our meals, met me at campus at 8am every morning, and stayed with me until we could barely move (usually around 10 or $11 \mathrm{pm}$ ). Thanks to their help, two weeks of testing were completed in four days. I love my parents to death and I will never forget how much they love me. 


\section{TABLE OF CONTENTS}

Page

LIST OF TABLES $\quad$ ix

LIST OF FIGURES $\quad$ x

LIST OF SYMBOLS X xii

\section{CHAPTER}

1 Introduction 1

1.1 Delaminations in Composites . . . . . . . . . . . . . . . . 1

1.2 Literature Review . . . . . . . . . . . . . . . . 5

1.2.1 Mode I Fracture in Unidirectional Composites . . . . . . . . . . . . 6

1.2.2 Mode I Fracture in Woven Fabric Composites . . . . . . . . . . . . . 9

1.2.3 Data Reduction Methods for the Double Cantilever Beam Test . . . 14

1.2.4 Post-Bond Delamination Resistance . . . . . . . . . . . . . . . . . 19

1.2.5 Summary of Literature Review Topics . . . . . . . . . . . . . . . 20

1.3 Specific Research . . . . . . . . . . . . . . . . . . . . 22

2 Mode I Composite Fracture Analysis 23

2.1 Introduction . . . . . . . . . . . . . . . . . . . 23

2.2 Area Method . . . . . . . . . . . . . . . . . . . 25

2.3 Modified Beam Theory . . . . . . . . . . . . . . . . 26

2.3.1 General Form of the Strain Energy Release Rate . . . . . . . . . . . 26

2.3.2 Modified Beam Theory _. . . . . . . . . . . . . . 29

2.4 Compliance Calibration Method _. . . . . . . . . . . . . . . 32

2.5 Modified Compliance Calibration Method . . . . . . . . . . . . . 33

2.6 Equivalent Stiffness Method . . . . . . . . . . . . . . . . . . . . . . . 35

2.7 Large Displacement Correction . . . . . . . . . . . . . . . . . 39

3 Experimental Design $\quad 40$

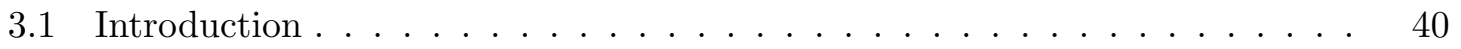

3.2 Material . . . . . . . . . . . . . . . . . . . . 40

3.3 Tensile Testing . . . . . . . . . . . . . . . . . . . . . . . 42

3.3.1 Specimen Preparation . . . . . . . . . . . . . . . . . 42

3.3.2 Machinery and Instrumentation . . . . . . . . . . . . . . . . . 44

3.3.3 Test Procedure . . . . . . . . . . . . . . . . . . . 45

3.3.4 Calculations. . . . . . . . . . . . . . . . . . . . . 45

3.4 In-Plane Shear Testing . . . . . . . . . . . . . . . . 47 
3.4.1 Specimen Preparation . . . . . . . . . . . . . . . . 47

3.4 .2 Machinery and Instrumentation . . . . . . . . . . . . . . . . . 49

3.4 .3 Test Procedure . . . . . . . . . . . . . . . . . . . . . . . . 49

3.4 .4 Calculations . . . . . . . . . . . . . . . . . . . . . 49

3.5 Short Beam Shear Testing . . . . . . . . . . . . . . . . . . . 50

3.5.1 Specimen Preparation . . . . . . . . . . . . . . . . . . . 52

3.5.2 Machinery and Instrumentation . . . . . . . . . . . . . 53

3.5.3 Test Procedure . . . . . . . . . . . . . . . . . . . 53

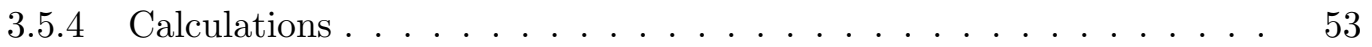

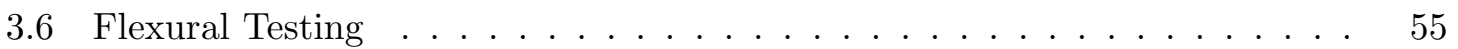

3.6.1 Specimen Preparation . . . . . . . . . . . . . 56

3.6 .2 Machinery and Instrumentation . . . . . . . . . . . . . . . 57

3.6 .3 Test Procedure . . . . . . . . . . . . . . . . . . . . 58

3.6.4 Calculations . . . . . . . . . . . . . . . 58

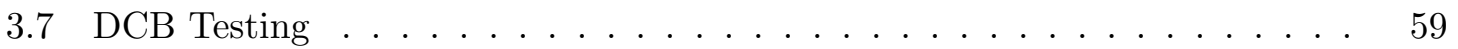

3.7.1 Specimen Preparation . . . . . . . . . . . . . . . . 59

3.7.2 Machinery and Instrumentation . . . . . . . . . . . . . . . 60

3.7.3 Test Procedure . . . . . . . . . . . . . . . . . . . 62

3.7 .4 Calculations . . . . . . . . . . . . . . 63

3.8 Summary of Testing Inputs and Outputs . . . . . . . . . . . . 63

4 Testing Results and Discussion 65

4.1 Tensile and In-Plane Shear Testing . . . . . . . . . . . . . . 65

4.2 Short Beam Shear Testing . . . . . . . . . . . . . . . . . . . . 67

4.3 Flexural Testing . . . . . . . . . . . . . . . . . . 69

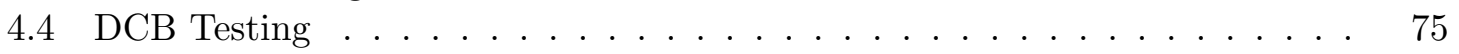

4.4.1 Post-Bond vs. Co-Cure Mode I Fracture . . . . . . . . . . . . . . . . . . 79

4.4.2 Direction Dependence for Mode I Fracture . . . . . . . . . . . . . . 82

4.4.3 Data Reduction and EQS Applicability . . . . . . . . . . . . 85

4.4.4 Applicability of Alternate Delamination Insertion Method . . . . . . 88

5 Conclusion $\quad 90$

5.1 Conclusions . . . . . . . . . . . . . . . . . . . . . 90

$5.2 \quad$ Future Studies . . . . . . . . . . . . . . . . . . . . 92

BIBLIOGRAPHY 95

\section{APPENDICES}

$\begin{array}{lr}\text { A Four-Point Bend Schematics } & 100\end{array}$

A.1 Four-Point Base . . . . . . . . . . . . . . . . . . . . . 101

A.2 Four-Point Standoff . . . . . . . . . . . . . . . . . . 102

B DCB Specimen Dimensions 103

B.1 Tensile Specimen Dimensions ． . . . . . . . . . . . . . . . . . . 104

B.2 In-Plane Shear Specimen Dimensions . . . . . . . . . . . . . . . . . 104

B.3 Short Beam Shear Specimen Dimensions . . . . . . . . . . . . . . . . . 104

B.4 Flex Specimen Dimensions . . . . . . . . . . . . . . . . . . . . . 105

B.5 DCB Specimen Dimensions . . . . . . . . . . . . . . . . 105 
$\begin{array}{ll}\text { C Hinge Schematics } & 106\end{array}$

C.1 Upper-Jaw Hinge . . . . . . . . . . . . . . . . . . . . . . . . . . 107

C.2 Bondable Hinges . . . . . . . . . . . . . . . . . . . . . . . . 108

C.3 Lower-Jaw Hinge . . . . . . . . . . . . . . . . . . . . . . . . . . . . 109

C.4 Pins . . . . . . . . . . . . . . . . . . 110

D Microscope Mount $\quad 111$

D.1 Microscope Mount Details . . . . . . . . . . . . . . . . . . . . . . 112

E Load Cell Calibration $\quad 113$

E.1 Calibration Procedure for LCH-100 Load Cell . . . . . . . . . . . . . . . . . 114

F DCB Specimen Load-Displacement Plots 116

F.1 $\quad P-\delta$ Plots for Plate $6 \ldots \ldots \ldots \ldots \ldots \ldots$

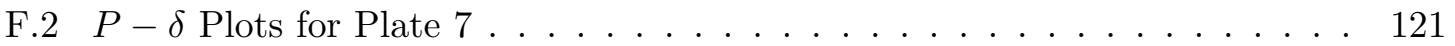

F.3 $\quad P-\delta$ Plots for Plate $8 \ldots \ldots \ldots \ldots \ldots \ldots$

F.4 $\quad P-\delta$ Plots for Plate $9 \ldots \ldots \ldots \ldots \ldots \ldots$

F.5 $\quad P-\delta$ Plots for Plate $10 \ldots \ldots \ldots \ldots \ldots \ldots$

F.6 $\quad P-\delta$ Plots for Plate $11 \ldots \ldots \ldots \ldots \ldots \ldots$

G DCB Specimen R-Curve Plots 139

G.1 R-Curves for Plate $6 \ldots \ldots \ldots \ldots \ldots$. . . . . . . . . . . . . . . . . . . .

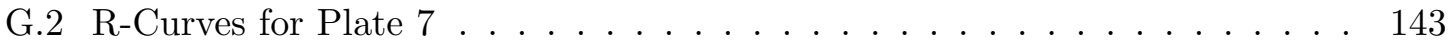

G.3 R-Curves for Plate $8 \ldots \ldots \ldots \ldots \ldots$

G.4 R-Curves for Plate $9 \ldots \ldots \ldots \ldots \ldots$. . . . . . . . . . . . . . . . . . . . .

G.5 R-Curves for Plate $10 \ldots \ldots \ldots \ldots \ldots$. . . . . . . . . . . . . . 152

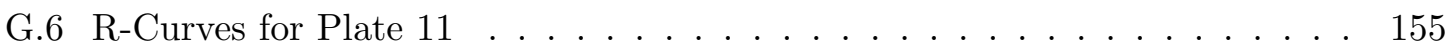

H DCB Data Reports $\quad 158$

H.1 Plate 6 Data Report . . . . . . . . . . . . . . . . . . . . 159

H.2 Plate 7 Data Report . . . . . . . . . . . . . . . . . 166

H.3 Plate 8 Data Report . . . . . . . . . . . . . . . . . . . . . . 172

H.4 Plate 9 Data Report . . . . . . . . . . . . . . . . . . . . 179

H.5 Plate 10 Data Report . . . . . . . . . . . . . . . . . . . . 185

H.6 Plate 11 Data Report . . . . . . . . . . . . . . . . . . . . . . 192 


\section{LIST OF TABLES}

1.1 Composite material properties . . . . . . . . . . . . . . . . 2

1.2 Relevant ASTM standards . . . . . . . . . . . . . . . . . 5

1.3 Fracture toughness for each region in Nicholls and Gallagher's study . . . . 8

3.1 Plate numbering . . . . . . . . . . . . . . . . . . . . . . 42

3.2 Tensile specimen average dimensions (inches) . . . . . . . . . . . . . 43

3.3 In-plane shear specimen average dimensions (inches) . . . . . . . . . . . 48

3.4 Short beam shear specimen average dimensions (inches) . . . . . . . . 53

3.5 Flex specimen average dimensions (inches) . . . . . . . . . . . . . . . . . 57

3.6 Inputs and outputs for each test series . . . . . . . . . . . . . . . 64

4.1 Tensile results . . . . . . . . . . . . . . . . . . 65

4.2 In-plane shear results . . . . . . . . . . . . . . . . . . 65

4.3 Tensile and in-plane shear statistics . . . . . . . . . . . . . . 67

4.4 Short beam shear data analysis . . . . . . . . . . . . . . 70

4.5 Flexural modulus data analysis . . . . . . . . . . . . . . . . 74

4.6 Flexural modulus data analysis . . . . . . . . . . . . . . . . 75

4.7 Comparisons between post-bonded and co-cured laminates . . . . . . . . . . 81

4.8 Non-linear crack initiation for traditional and alternate delamination insertion methods . . . . . . . . . . . . . . . . . . 89

5.1 Example test series for post-bond/co-cure comparisons . . . . . . . . . . . 93

5.2 Example test series for delamination direction comparisons . . . . . . . 93

5.3 Example test series for delamination insertion comparisons . . . . . . . . 93

B.1 Tensile specimen average dimensions (inches) . . . . . . . . . . . . . 104

B.2 In-plane shear specimen average dimensions (inches) . . . . . . . . . . . 104

B.3 Short beam shear specimen average dimensions (inches) . . . . . . . . . 104

B.4 Flex specimen average dimensions (inches) . . . . . . . . . . . . . . . 105

B.5 DCB specimen average dimensions (inches) . . . . . . . . . . . 105 


\section{LIST OF FIGURES}

1.1 (i): Print through of a co-cured 5/8" thick foam core within a roll bar test specimen at the California Polytechnic State University in San Luis Obispo (ii): Post-Bonded frame mount inside a composite fairing for a Human Pow-

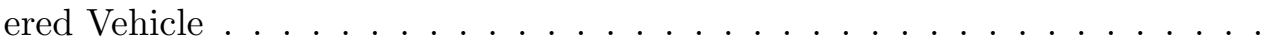

1.2 The three modes of fracture: (i): Opening of crack (mode I), (ii): In-Plane shear of crack (mode II), (iii): Anti-Plane shear of crack (mode III) . . . . . 4

1.3 Double cantilever beam (DCB) specimen . . . . . . . . . . . . . 7

1.4 (i): Plain weave cloth section (one over, one under) (ii): Cloth appearance 10

1.5 (i): Twill weave (over two, under one) (ii): Cloth appearance . . . . . . . 10

1.6 (i): 8-Harness-Satin weave (over seven, under one) (ii): Cloth appearance . 11

1.7 (i): Plain weave, $n_{g}=2$ (ii): Twill weave, $n_{g}=3$ (iii): Basket weave, $n_{g}=4$ (iv): 8 -Harness-Satin weave, $n_{g}=8(\mathrm{v})$ : Reverse side of the 8-Harness-Satin weave . . . . . . . . . . . . . . . . . . . . . 12

1.8 (i): Transverse yarn debonding (ii): Free fill and weft yarns . . . . . . . 13

1.9 (i): Typical load-displacement $(P-\delta)$ curve (ii): Corresponding fracture resistance $\operatorname{spread}[6] \ldots \ldots \ldots \ldots \ldots \ldots$

2.1 DCB test specimen dimensions . . . . . . . . . . . . . . . . 24

$2.2 \quad P-\delta$ trace for area method calculations . . . . . . . . . . . 25

2.3 Griffith Crack: A through-thickness crack in a uniaxially loaded plate of

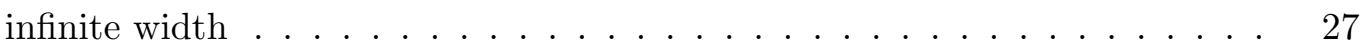

2.4 LEFM example $P-\delta$ plot $[32] \ldots \ldots \ldots \ldots \ldots \ldots$

2.5 Basic beam diagram for modified beam theory calculations . . . . . . . 29

2.6 MBT crack length correction term . . . . . . . . . . . . . . . 31

2.7 Compliance calibration experimentally determined coefficient . . . . . . . 33

2.8 Modified compliance calibration experimentally determined coefficient . . . 34

2.9 Anticlastic bending of a beam . . . . . . . . . . . . . . . . . . . . 36

2.10 DCB beam bending specimen . . . . . . . . . . . 37

3.1 Autoclave cure cycle . . . . . . . . . . . . . . . . . . 41

3.2 Definition of the principal material directions for this study. Note: $1 \leftrightarrow$ Longitudinal and $2 \leftrightarrow$ Transverse . . . . . . . . . . . . . . . . . . . . . . 41

3.3 Typical tensile coupon geometry . . . . . . . . . . . . . . . 43

3.4 Symmetric laminate structure for harness satin weaves . . . . . . . . . . . 44

3.5 Strain gage locations for the tensile test specimens . . . . . . . . . . . . 44

3.6 Poisson's ratio for longitudinal tensile test . . . . . . . . . . . . . 45

3.7 Extrapolation for ultimate longitudinal tensile failure strain . . . . . . . . 46

3.8 Typical $\pm 45^{\circ}$ shear coupon geometry . . . . . . . . . . . . . . . 48

3.9 Strain gage locations for the in-plane shear test specimens . . . . . . . . . 48 
3.10 The Shear Modulus, $G_{12}$, from the in-plane shear test . . . . . . . . . 51

3.11 Shear and bending moment diagram for three-point bend test . . . . . . . . 52

3.12 Typical short beam shear specimen geometry . . . . . . . . . . . . 52

3.13 Beam section through $\mathrm{q} \ldots \ldots \ldots \ldots \ldots$

3.14 Comparison of three and four point shear and bending moment diagrams . 56

3.15 Typical geometry for flex specimens . . . . . . . . . . . . 56

3.16 LVDT calibration chart . . . . . . . . . . . . . . . 58

3.17 Typical geometry for DCB specimens . . . . . . . . . . . . . 60

3.18 Laser-engraved crack propagation markings . . . . . . . . . . . . . 62

4.1 Longitudinal modulus of elasticity in the fiber direction . . . . . . . . 66

4.2 Major Poisson's ratio, $\nu_{12}$, from longitudinal pull . . . . . . . . . . 66

4.3 Composite in-plane shear modulus . . . . . . . . . . . . . . 67

4.4 Short beam shear inelastic deformation failure mode . . . . . . . . . . 68

4.5 Short beam shear flexure failure mode . . . . . . . . . . . . . 69

4.6 Short beam shear interlaminar shear failure mode . . . . . . . . . . . . 69

$4.7 \quad P-\delta$ curve for plate 12 SBS specimens $\ldots \ldots \ldots \ldots \ldots$

$4.8 \quad P-\delta$ curve for plate 13 SBS specimens $\ldots \ldots \ldots \ldots \ldots \ldots$

$4.9 P-\delta$ curve for plate 12 flex specimens . . . . . . . . . . . . 71

$4.10 P-\delta$ curve for plate 13 flex specimens . . . . . . . . . . . . . 72

4.11 Flexural modulus for plate 12 (co-cured) . . . . . . . . . . . . . . . . . 73

4.12 Flexural modulus for plate 13 (post-bonded) . . . . . . . . . . . . 73

4.13 Typical failure mode for flex specimens under four point bending . . . . . . 74

4.14 Generated R-curve for specimen $10-6 \ldots \ldots \ldots$

$4.15 P-\delta$ trace for specimen $10-6 \ldots \ldots \ldots \ldots$

4.16 Non-linear propagation verification and could be evidence of matrix plastic deformation . . . . . . . . . . . . . . . . . . 79

4.17 Out of plane crack growth due to the complex structure of WFCs in $90^{\circ}$

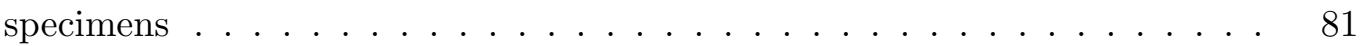

4.18 Typical crack tip progression in $0^{\circ}$ specimens $\ldots \ldots \ldots$. . . . . . . 82

4.19 Difference between $90^{\circ}$ (left) and $0^{\circ}$ (right) R-curves . . . . . . . . . . 83

E.1 First calibration weight . . . . . . . . . . . . . . . . . 114

E.2 Second calibration weight . . . . . . . . . . . . . 115

E.3 Third calibration weight . . . . . . . . . . . . . . . 115 


\title{
LIST OF SYMBOLS
}

\author{
$\Delta \quad$ Delamination Extension Correction \\ $\delta \quad$ Crosshead Displacement \\ $\epsilon \quad$ Normal Strain \\ $\epsilon_{m}^{o} \quad$ Midplane Strain Vector \\ $\epsilon_{1} \quad$ Longitudinal Normal Strain \\ $\epsilon_{2} \quad$ Transverse Normal Strain \\ $\epsilon_{x} \quad$ Normal Strain in x-Direction \\ $\epsilon_{y} \quad$ Normal Strain in y-Direction \\ $\epsilon_{1 t}^{u} \quad$ Ultimate Longitudinal Tensile Failure Strain \\ $\epsilon_{2 t}^{u} \quad$ Ultimate Transverse Tensile Failure Strain \\ $\gamma_{12} \quad$ In-Plane Shear Strain \\ $\gamma_{12}^{u} \quad$ Ultimate In-Plane Shear Strain \\ $\gamma_{x y} \quad$ Shear Strain in $\mathrm{x}-\mathrm{y}$ Coordinates \\ $\kappa_{q} \quad$ Midplane Curvature Vector \\ $\kappa_{x} \quad$ Plate Curvature in $\mathrm{y}-\mathrm{z}$ Plane \\ $\kappa_{y} \quad$ Plate Curvature in $\mathrm{x}-\mathrm{z}$ Plane \\ $\kappa_{x y} \quad$ Twist of Plate \\ $[T]_{1} \quad$ Stress Transformation Tensor \\ $[T]_{2} \quad$ Strain Transformation Tensor \\ $\nu_{12} \quad$ Major Poisson's Ratio \\ $\nu_{21} \quad$ Minor Poisson's Ratio \\ $\overline{E I}$ Equivalent Stiffness for the EQS Method \\ $\Pi \quad$ Total Potential Energy \\ $\rho \quad$ Mass Density
}




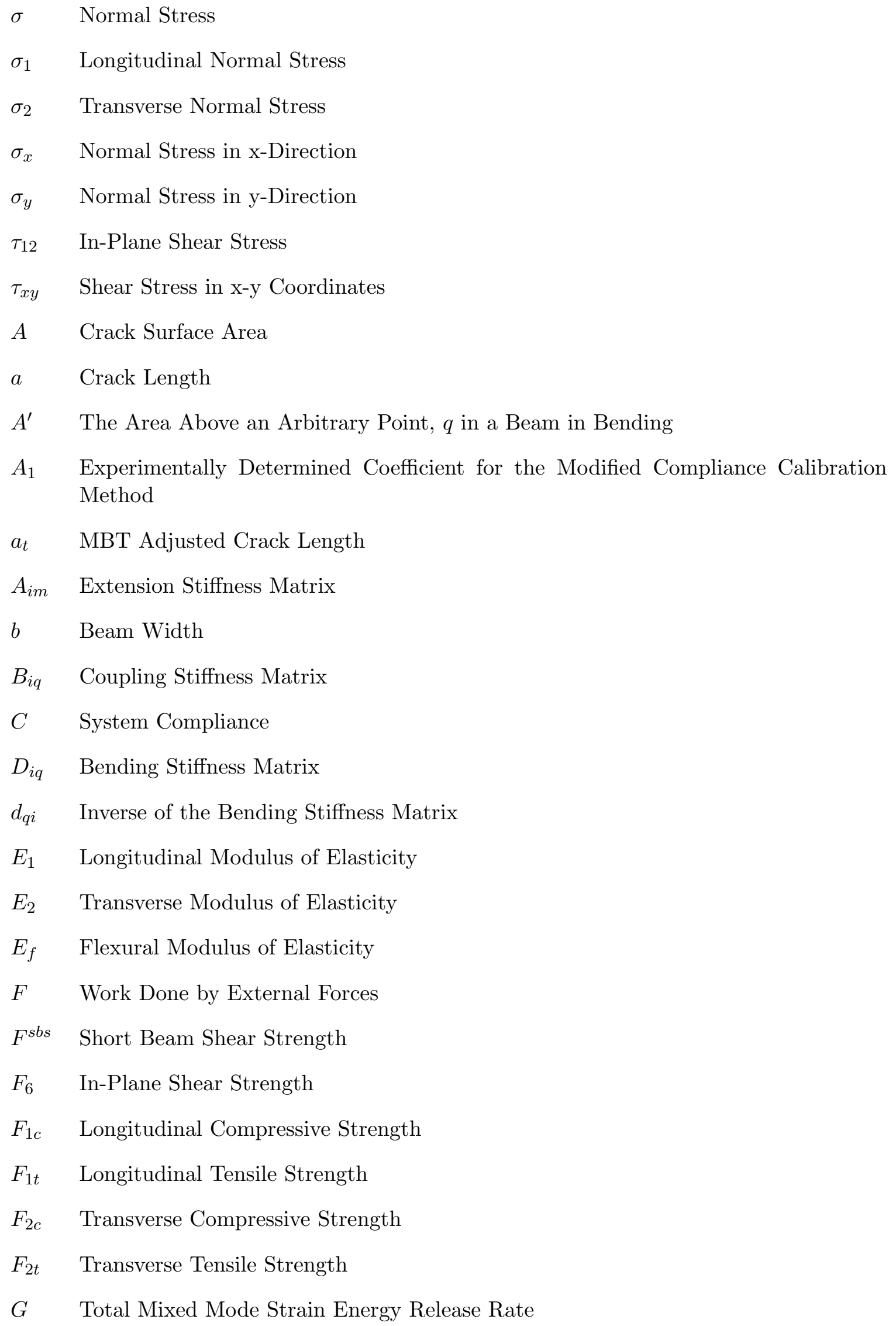


$G_{c} \quad$ Mixed Mode Fracture Toughness

$G_{I} \quad$ Mode I Strain Energy Release Rate

$G_{12} \quad$ In-Plane Shear Modulus

$G_{\text {Ic }} \quad$ Mode I Fracture Toughness

$G_{I I}$ Mode II Strain Energy Release Rate

$H \quad$ Experimentally Determined Coefficient for the Compliance Calibration Method

$h \quad$ DCB Specimen Thickness

I Moment of Inertia

$K_{I} \quad$ Mode I Stress Intensity Factor

$L \quad$ Length of Tensile Test Specimen

$L_{s} \quad$ Span Width for Three and Four-Point Bending

$M_{i} \quad$ Line Moment Vector

$M_{x} \quad$ Line Moment in x-Direction

$M_{y} \quad$ Line Moment in y-Direction

$M_{x y} \quad$ Line Moment in xy-Direction

$n \quad$ Experimentally Determined Coefficient for the Compliance Calibration Method

$n_{g} \quad$ Weave Index

$N_{i} \quad$ Line Load Vector

$P \quad$ Applied Load on DCB Specimen

$q \quad$ An Arbitrary Point in the Cross Section of a Beam in Bending

$U \quad$ Total Strain Energy Stored in Test Specimen

$V \quad$ Vertical Shear Force

$z^{\prime} \quad$ The Distance from the Neutral Axis of a Beam in Bending to $A^{\prime}$

E Modulus of Elasticity 


\section{Chapter1}

\section{Introduction}

\subsection{Delaminations in Composites}

Composite materials such as Kevlar ${ }^{\circledR} /$ Epoxy and Carbon/Epoxy have become incredibly popular in many engineering disciplines in the past several decades due to their high strength and stiffness to weight ratios and ability to conform easily to complex shapes. Composite structures are normally composed of an assemblage of many layers, or laminae, of material. This assemblage is known as a laminate and can be created to meet the stiffness and strength requirements of almost any structure. The laminate, however, does not react to applied loads in the same manner as most other materials. A composite laminate, in many cases, cannot be treated as an isotropic material. Any given unit of the composite laminate would have unique material properties in at least two different directions. A unit of another well known material, steel for instance, can be treated as though it has the same material properties in every direction.

The non-isotropy of composite structures makes them more difficult to design than those composed of isotropic materials. Properties in each unique material-direction must be determined through experimental testing. These properties are then used along with Composite Laminate Theory (CLT) to determine laminate stresses and strains in 2D space. Many modern finite element softwares incorporate CLT to aid in composite design which dramatically decreases the amount of time required to analyze composite structures. 
Table 1.1: Composite material properties

\begin{tabular}{ll}
\hline \hline Symbol & Definition \\
\hline$E_{1}$ & Longitudinal Modulus of Elasticity \\
$E_{2}$ & Transverse Modulus of Elasticity \\
$\nu_{12}$ & Major Poisson's Ratio \\
$\nu_{21}$ & Minor Poisson's Ratio \\
$G_{12}$ & In-Plane Shear Modulus \\
$\rho$ & Mass Density \\
$F_{1 t}$ & Longitudinal Tensile Strength \\
$F_{2 t}$ & Transverse Tensile Strength \\
$F_{6}$ & In-Plane Shear Strength \\
$\epsilon_{1 t}^{u}$ & Ultimate Longitudinal Tensile Failure Strain \\
$\epsilon_{2 t}^{u}$ & Ultimate Transverse Tensile Failure Strain \\
$\gamma_{12}^{u}$ & Ultimate In-Plane Shear Strain \\
\hline
\end{tabular}

The properties listed in Table 1.1 are some of the most desired material properties and work well for many $2 \mathrm{D}$ composite structures under static or quasi-static loading. Imperfections such as notches, cracks, debris which entered the laminate during the layup process, or from damage during the installation or maintenance of the composite structure (i.e. impact) can cause a laminate to perform in an unpredictable manner. One of the failures most commonly witnessed from these types of imperfections is a separation of the lamina, also known as delamination. The use of safety factors or special composite construction like edge strengthening or modification, as described in [13, Chapter 8], can mitigate the effect of these imperfections to artificially increase the laminate's resistance to delamination. This form of mechanical delamination resistance may not always be an option, however, and does not necessarily guarantee structural resistance to dynamic loading.

Delamination may be especially prevalent in cases where laminates have been formed in post-bond (co-bonding) operations. A post bond is the curing of one laminate to another that is already fully cured without the use of any additional adhesive. In most cases, a light sanding followed by a cleaning with acetone or denatured alcohol is considered enough to provide a good bond. The goal of this surface preparation is to increase the likelihood of a molecular bond and raise the surface-free energy thus allowing the matrix material to spread more evenly across the surface rather than beading up [36]. Surface treatment is not 
enough to produce a bond equal to the one between laminate plies that are all cured at the same time (co-cured), meaning post-bonded joints will have delamination resistances lower than those of conventional composite laminates. Post-bonds are very common in composite structures, particularly when print-through of a core material is not desired (see Figure 1.1) or when a structure's complicated geometry requires additional composite components to be added after the initial cure.

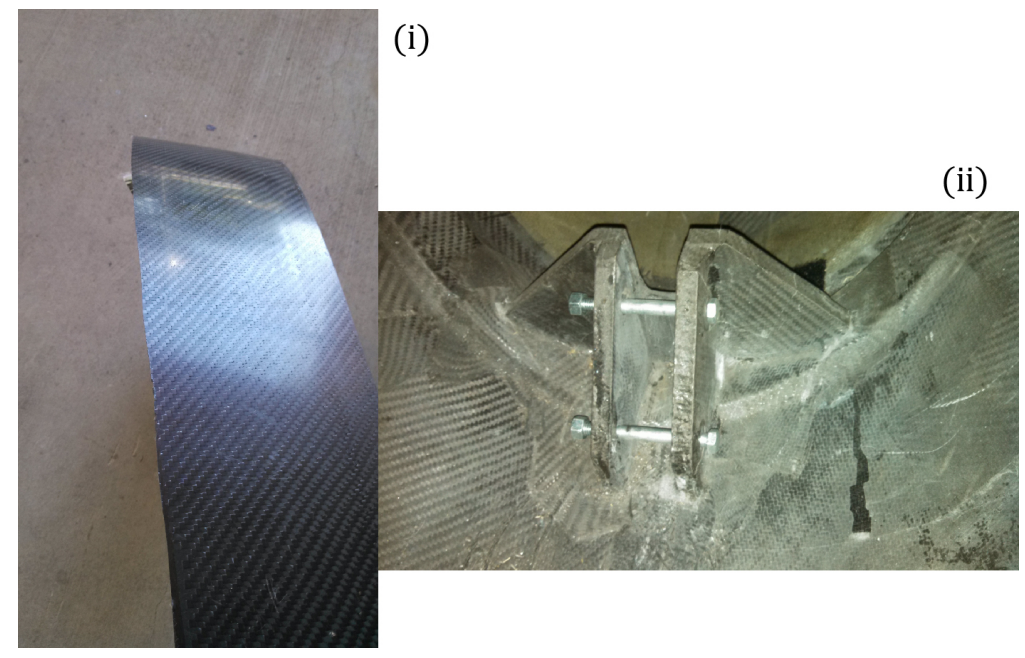

Figure 1.1: (i): Print through of a co-cured 5/8" thick foam core within a roll bar test specimen at the California Polytechnic State University in San Luis Obispo (ii): PostBonded frame mount inside a composite fairing for a Human Powered Vehicle

A delamination is a propagation of interlaminar defects which can result in a catastrophic loss of structural stiffness. A plane wing is one example of a composite structure which can be subjected to forces which may cause delamination. Undue consideration of the possibility of delamination during the design of the laminates forming the wing could lead to a dramatic failure during flight. Another common structure which can be made of composite materials is a bicycle frame. Delamination should be considered during the design of the frame in order to ensure this failure will not occur from normal use. Serious injuries, and even death, could occur if the laminates comprising the frame were to delaminate. Delamination can be difficult to diagnose because it normally occurs at the interior of a laminate and is not visible on the outside of a structure; it can, however, be characterized and used during 
the design process. Characterization can be done by determining the fracture toughness, $G_{I c}\left(K_{I c}\right.$ is also commonly used), through either of two main approaches: a stress based approach aimed at finding the stress-intensity factor, $K_{I}$, or an energy approach aimed at determining the strain energy release rate, $G_{I}$. Once a composite system's resistance to delamination is characterized, the laminate can be optimized to withstand the loads to which it will be subjected.

Aside from edge strengthening and stiffening, one of the easiest ways to increase delamination resistance in a composite is to toughen the resin. One main reason to utilize standardized fracture tests is the ability to evaluate new resin types for their capability to resist fracture [34]. In addition to determining values of $G_{I c}$ for design purposes, standardized tests also allow one to correlate damage tolerance to delamination resistance for the assessment of structural integrity of long-life composite structures.

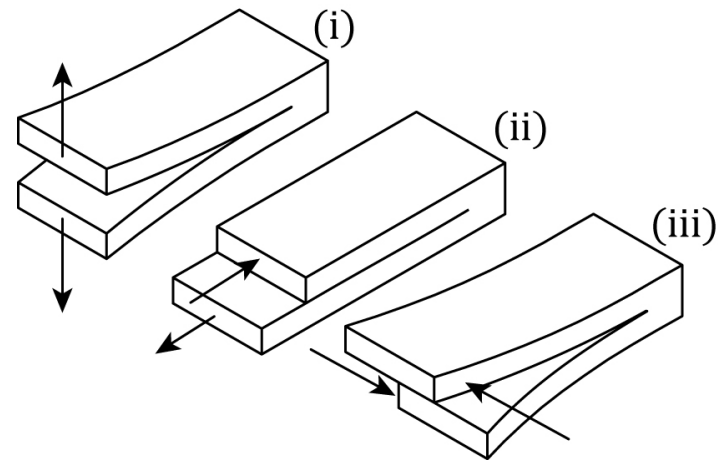

Figure 1.2: The three modes of fracture: (i): Opening of crack (mode I), (ii): In-Plane shear of crack (mode II), (iii): Anti-Plane shear of crack (mode III)

Delamination is modeled as one of three independent fracture modes: Modes I, II, and III, corresponding to opening of the crack (delamination), in-plane shear of the crack, and anti-plane shear of the crack, respectively, as shown in Figure 1.2. While test methods for the values listed in Table 1.1 have been well documented and standardized for quite some time, the standard test methods for mode I, II, and III, along with a mixed mode I/II fracture are still under scrutiny. The only ASTM standards relating, specifically, to fracture 
in composite materials are D5528 [6] and D6671 [4] for mode I and mode I/II fracture, respectively, as listed in Table 1.2 .

Table 1.2: Relevant ASTM standards

\begin{tabular}{ll}
\hline \hline Standard & Properties Determined \\
\hline ASTM D3518 & $G_{12}$ \\
ASTM D3039 & $E_{1}, E_{2}, \nu_{12}, \nu_{21}, F_{1 t}, F_{2 t}, \epsilon_{1 t}^{u}, \epsilon_{2 t}^{u}$ \\
ASTM D5528 & $G_{I}, G_{I c}$ \\
ASTM D6671 & $G_{I}, G_{I I}, G, G_{c}$ \\
JIS K7086 & $G_{I}, G_{I c}, G_{I I}, K_{I I c}$ \\
$\operatorname{prEN~6033~}$ & $G_{I}, G_{I c}$ \\
$\operatorname{prEN~6034}$ & $G_{I I}, K_{I I c}$ \\
\hline
\end{tabular}

Mode I fracture is of particular interest here and will be the only mode discussed further. There are many restrictions in the ASTM Standard regarding the type of composite that should be tested. Unidirectional composites, in particular, are specified as being the preferred laminae in pure mode I testing with a delamination propagating in the fiber direction at the mid-plane of a laminate. Many structures, however, are composed of woven cloth composite laminae whose fracture resistance is a determining factor in the structure's design. The direction of the crack propagation is another factor that varies between laminate orientations and loading conditions. A crack may not, for instance, propagate along a fiber direction if an imperfection simulates a pre-existing delamination normal to the fibers. Many other factors limit the applicability of mode I standards to realistic design cases and will be discussed further in the following chapters.

\subsection{Literature Review}

The study of delamination in composite materials has evolved considerably since the first test conducted in 1980 [18]. Variations include testing parameters (i.e. load rate, specimen dimensions, delamination films, etc), stacking sequence, methods for the calculation of fracture toughness, the orientation of the fibers, different materials, and many others. Characterization of the modes of delamination has been performed through the use of scanning electron microscopy (SEM). Features like fiber bridging, matrix deformation, and 
fiber/matrix adhesion failure can be identified through the use of SEM in an effort to judge the applicability of the assumptions made in the calculations for fracture toughness. The following literature review will focus on several main topics viz. Mode I fracture in unidirectional composites, mode I fracture in woven fabric composites, alternate methods for the calculation of fracture toughness, and post-bond delamination resistance. Several of the above topics will include the effects of differing delamination insertion methods and the relationship between fracture toughness and delamination direction.

\subsubsection{Mode I Fracture in Unidirectional Composites}

Most of the research concerning fracture in composite materials has been focused on unidirectional fabrics (tape). In fact, ASTM D5528 specifically recommends the use of these fabrics in mode I fracture testing. The most common mode I test is the Double Cantilever Beam (DCB) test depicted in Figure 1.3. The specimen consists, effectively, of two cantilever beams, fixed to each other in the uncracked region of the beam. The effective length of the cantilever beam is the longitudinal distance between the center of the pin and the crack tip.

The DCB test first became popular for fracture testing of fiber reinforced composite materials in the 1980s. In 1982, Whitney et al. [69] studied the applicability of the DCB test in fracture toughness testing. They determined that the test was able to differentiate between materials with differing interlaminar toughness values, making it a useful tool for composite analysis. In 1984, Chai [24] performed DCB tests on multidirectional laminates with both brittle and ductile resin systems. Crack propagation did not remain in the initial plane and tended to move between different ply interfaces, as is common in DCB testing. Other frequently observed phenomena were crack jumping, fiber pull-out, and fiber breakage. Some areas along the crack were free of these imperfections and Chai was able to use data from these regions to calculate the energy release rate for multiple ply orientations. He concluded that the fracture toughness is independent of relative laminae orientation. 


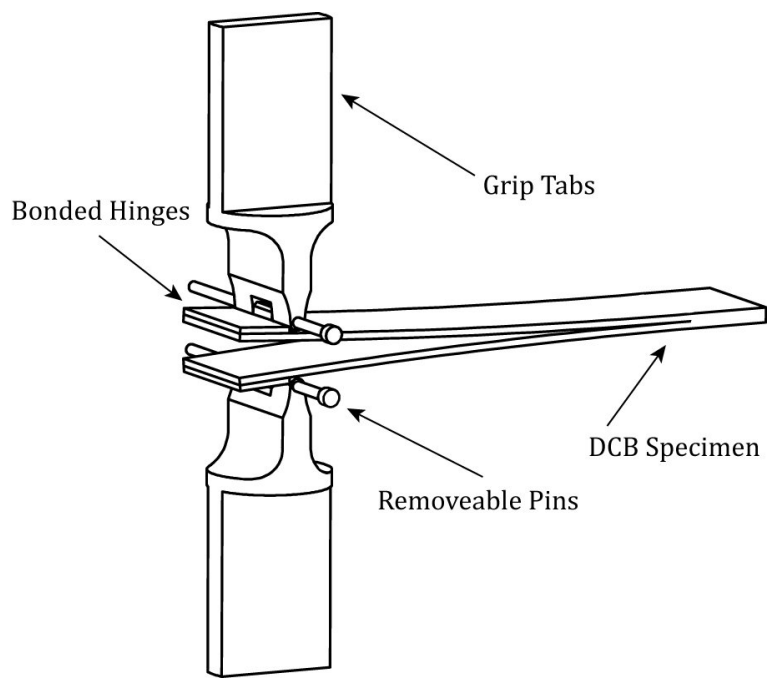

Figure 1.3: Double cantilever beam (DCB) specimen

Russel and Street [60] performed tests on AS1/3501-6 Graphite/Epoxy to determine the mode I fracture toughness at $0^{\circ} / 0^{\circ}, 45^{\circ} /-45^{\circ}$ and $90^{\circ} / 90^{\circ}$ relative midplane ply orientations. The results indicated a negligible spread in $G_{I c}$ initiation values (first crack to occur, corresponding to the end of the delamination insert). The steady state values of $G_{I c}$, however, were two to three times smaller for the $0^{\circ} / 0^{\circ}$ interface than for the off-axis specimens, which was attributed to excessive "matrix crack branching and deviation." Bradley et al. [21] performed tests on $+45^{\circ} /-45^{\circ}$ and $0^{\circ} / 0^{\circ}$ interfaces. They noted that, when specific energy dissipation (see below) did not occur in undesired forms i.e. fiber breakage, fiber bridging, etc., mode I fracture toughness was similar for each layup.

Nicholls and Gallagher [53] performed tests on laminates of AS1/3502 Carbon Fiber/Epoxy with layup schedules of the form $\left[+\theta /-\theta / 0_{8} /-\theta /+\theta\right]_{\text {sym }}$ for $\theta=0^{\circ}, 15^{\circ}, 30^{\circ}, 45^{\circ}, 60^{\circ}$, and $90^{\circ}$. Four distinct regions of crack growth with different morphologies were identified. Region I was a smooth midplane fracture. Region II was an area where the crack had moved from the midplane and matrix deformation was apparent with a microscope. Region III was an area where the crack broke through one of the angle plies; matrix deformation was seen at lower angles while the crack split at higher angles. Region IV was where the crack propagated between a $0^{\circ}$ ply and another angled ply and revealed a rough surface consist- 
ing of ridges and valleys formed between fibers and matrix. Fracture toughness values for each region were distinct, as shown in Table 1.3, which indicates a dependency of fracture toughness on interface ply orientations and, likewise, crack growth directions with respect to principal material directions.

Table 1.3: Fracture toughness for each region in Nicholls and Gallagher's study

\begin{tabular}{cc}
\hline \hline Region & Fracture Toughness, $G_{I c}\left(\mathrm{~kJ} / \mathrm{m}^{2}\right)$ \\
\hline I & 0.14 for $\theta=0^{\circ}$ \\
II & 0.25 for $\theta=30^{\circ}-90^{\circ}$ \\
III & $0.25-0.82$ for $\theta=15^{\circ}-60^{\circ}$ \\
IV & 0.30 for $\theta=60^{\circ}$ and $90^{\circ}$
\end{tabular}

Robinson and Song [58] performed DCB tests on XAS-913C Carbon/Epoxy laminates with a 0.0004in delamination insert. They found that fracture is complicated by crack jumping and fiber bridging for any interface orientations other than $0^{\circ} / 0^{\circ}$. An edge delamination was incorporated in $+45^{\circ} /+45^{\circ}$ and $+45^{\circ} /-45^{\circ}$ laminates. This addition tended to keep the crack propagation through the desired interface and fracture toughness reached steady state values $28 \%$ higher than those calculated for the $0^{\circ} / 0^{\circ}$ specimens. Robinson and Song suggested that ply orientation does affect the fracture toughness of composite laminates. A comprehensive review of fracture toughness dependence on crack propagation direction can be found in [17]. The overall conclusion from this review is that brittle matrix composites appear to have a higher dependence on ply orientation than do high toughness (ductile) composites where mode I fracture is concerned.

The contributing factors to the complexity of $G_{I c}$ calculations are mode mixity, residual stresses, energy release rate distribution along the delamination front, and specific energy dissipation [17]. The effects of mode mixity, referring to multiple fracture modes acting at the same time, and the energy release rate distribution can both be relieved through choices in layup schedule and test setup as will be discussed in Chapters 2 and 3. Residual stresses refer, mainly, to thermal loads resulting from the cure cycle and can be neglected by using proper cure cycles as suggested by the material manufacturer. Specific energy dissipation 
refers to any form of energy dissipation not corresponding to in-plane crack propagation viz. crack jumping, fiber pull-out, fiber breakage, fiber bridging, matrix deformation, matrix cracking, crack branching, and crack deviation.

Aliyu and Daniel [16] studied the effects of varying the deflection rate at the load application end of the DCB in 1985. AS4/3501-6 Graphite/Epoxy was used with quasistatic deflection rates of $0.02 \mathrm{in} / \mathrm{min}$ up to $20 \mathrm{in} / \mathrm{min}$ with a 0.001 in Teflon ${ }^{\circledR}$ delamination insert. The results indicated that $G_{I c}$ increased with increasing deflection rates. This means that low deflection rates should be used to evaluate composite mode I fracture toughness in order to obtain more conservative results.

\subsubsection{Mode I Fracture in Woven Fabric Composites}

The study of mode I fracture in composite materials has been dominated by the testing of unidirectional laminates. Woven fabric composites (WFCs), on the other hand, have not received much attention and are still not well understood. These materials are far more complex than unidirectional composites (UDCs) and are not recommended by ASTM D5528 for mode I fracture testing. Unfortunately, structures composed of WFCs cannot be fully designed without some knowledge of their fracture performance. The complexity of WFCs arises from their macroscopic material structure. Whereas UDCs only have fibers running in one direction and, likewise, only in one plane, WFCs are composed of interwoven bundles of fibers which run in at least two directions and pass in and out of plane. A plain weave cloth section is shown in Figure 1.4. Note how the bundles of fibers (yarns) move between the top and bottom planes. The yarns in each direction can have unique material properties which would lead to unequal composite properties in the two principal directions, or they can be made of the same material, leading to equal composite properties in both principal directions.

Basket weave, as opposed to plain weave cloth, is composed of yarns woven in an over two, under two pattern to yield a more spread out lamina with fewer harsh bends in the yarns. The similarities between basket and plain weave are many; most importantly: they 

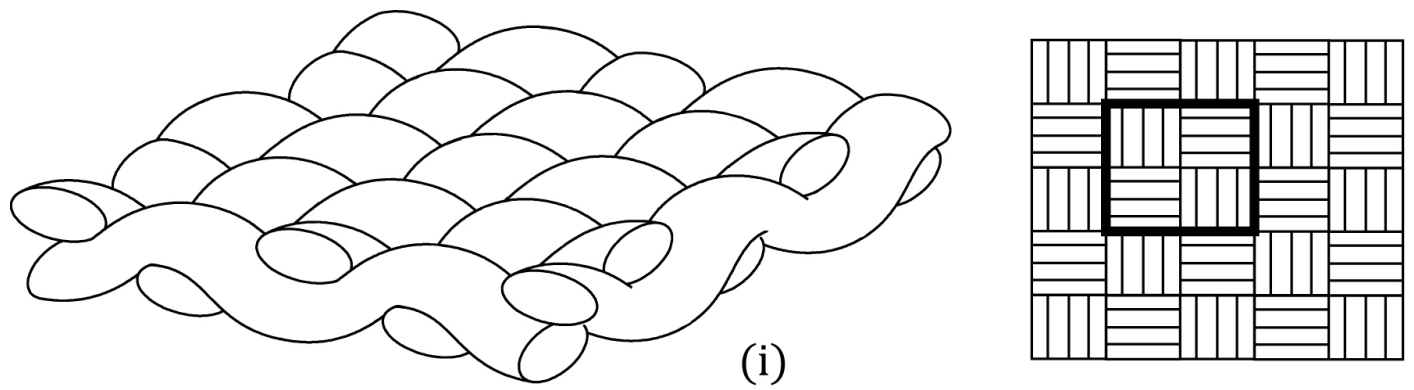

(ii)

Figure 1.4: (i): Plain weave cloth section (one over, one under) (ii): Cloth appearance
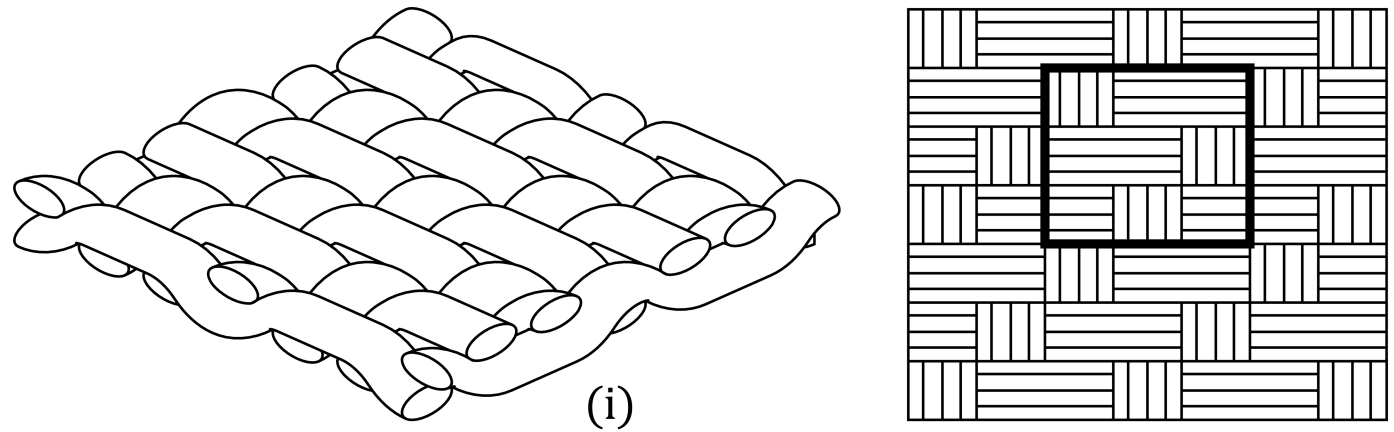

(ii)

Figure 1.5: (i): Twill weave (over two, under one) (ii): Cloth appearance

are symmetric about their midplanes. The fabric can be placed with either the top or bottom face down in a laminate without any structural consequences. These cloths also have an equal amount of fibers running in both directions on both sides of the laminae. WFCs are defined by the weave index, $n_{g}$. Plain weave has $n_{g}=2$ and basket has $n_{g}=4$. The weave index is a measure of how many yarns are passed under or over by a single yarn before a pattern is repeated and indicates the size of the unit cell. The unit cell, as outlined in Figure 1.4 (ii), 1.5 (ii), and 1.6(ii) is the smallest repeated block of material to make up the fabric as a whole.

Twill and harness-satin (HS) weaves do not share the same attributes of plain and basket weaves. They are not symmetric about their midplanes and do not have an equal number of fibers running in both principal directions on either side of the cloth. Instead, a yarn 
will pass over several other yarns and under one before the pattern is repeated. A twill fabric (see Figure 1.5) has yarns passing over two perpendicular yarns and under one, an 8-HS weave will have yarns passing over 7 perpendicular yarns and under one. Figure 1.6 shows an 8-HS cloth with its corresponding unit cell. Like plain and basket weave fabrics, the yarns in harness satin and twill weaves are not restricted to a single plane but travel between the top and bottom faces, leaving gaps which are commonly filled with resin (resin pockets). Twill and harness-satin weaves are also defined by the weave index. X-HS cloth has $n_{g}=X$ and twill weave fabrics have $n_{g}=3$. A comparison between unit cells for the different fabric types can be found in Figure 1.7.
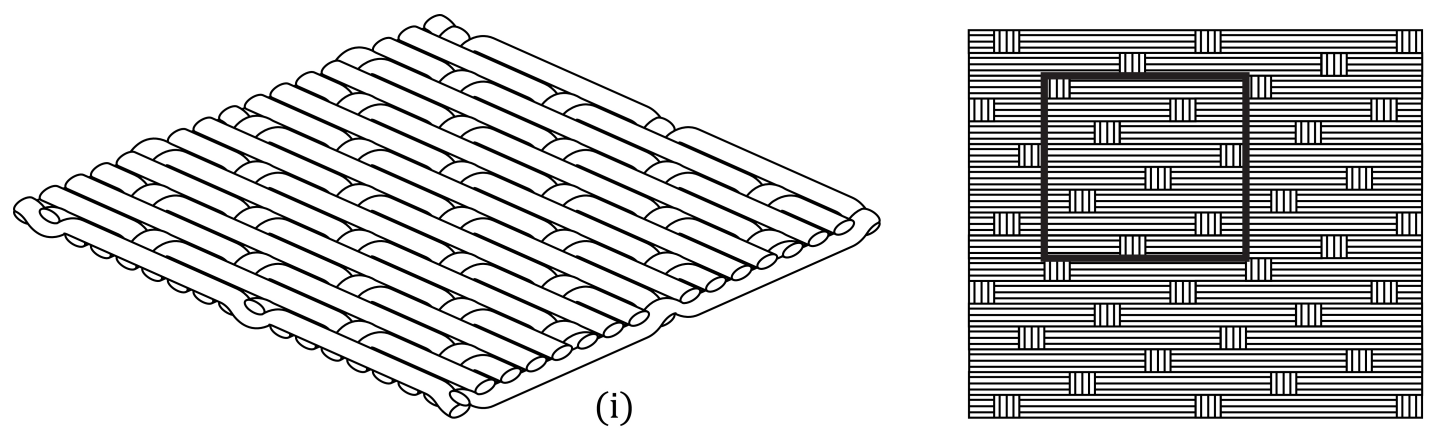

Figure 1.6: (i): 8-Harness-Satin weave (over seven, under one) (ii): Cloth appearance

It is important to note that the reverse side of the harness satin weaves is not the same as the front side. One side of the fabric contains yarns running predominately at $0^{\circ}$ (fill yarns) and the opposite side has yarns running predominately at $90^{\circ}$. The non-dominant yarns on each side of the cloth are known as weft yarns. Weft and fill yarns are fabric side-dependent; a weft yarn on one side of the fabric will be a fill yarn on the other. The structural difference between the two sides of the fabric will have an effect on the cloth's performance under mode I fracture conditions. The rate at which delaminations propagate, as well as the uniformity of the delamination, varies depending on the orientation of the interface plies. The studies discussed, below, suggest that if a DCB test specimen is made with the delamination interface plies oriented with fill yarns running in the direction of delamination growth, crack propagation will be met with significantly lower resistance 
than in a specimen with delaminations along weft yarns. It has been suggested $[31,66]$ that, since composite delamination is so affected by weave structure, it should be treated as a structural, rather than a material, property to try to account for changes in fracture resistance with differing crack growth directions.

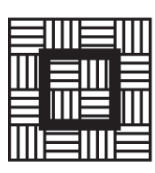

(i)

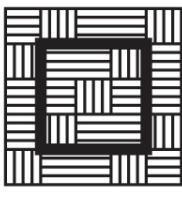

(ii)

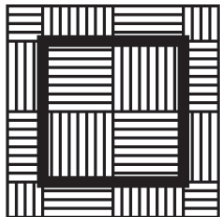

(iii)

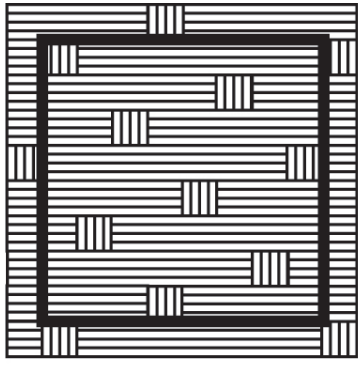

(iv)

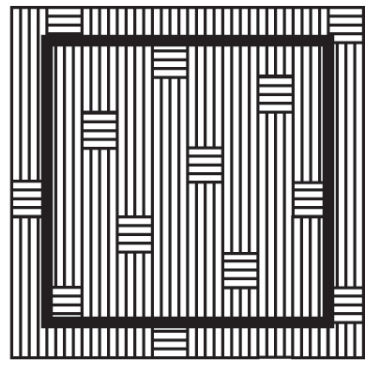

(

Figure 1.7: (i): Plain weave, $n_{g}=2$ (ii): Twill weave, $n_{g}=3$ (iii): Basket weave, $n_{g}=4$ (iv): 8-Harness-Satin weave, $n_{g}=8(\mathrm{v})$ : Reverse side of the 8-Harness-Satin weave

Naik et al. [51] evaluated critical strain energy release rates for mode I, II, I/II, and III fracture in four types of E-Glass/Epoxy, plain weave composites. Tests were performed with delaminations propagating at $0^{\circ}$ and $45^{\circ}$ to the fiber directions. It was observed that plain weave fabrics with smaller strand widths had higher resistance to fracture than plain weave fabrics with increased strand width. Delamination direction also had an effect on the fracture toughness, with a higher resistance to fracture recorded when the delamination propagated in the fiber direction rather than at $45^{\circ}$. In 1997, Burchill and Simpson [22] found that the fiber volume ratio, or the relative amount of fibers vs. matrix material, does not have an effect on the fracture toughness of the composite as long as the ratio is between $30 \%$ and $70 \%$. These outcomes further emphasize the possible need to treat composite delamination as a structural property.

Alif et al. [15] studied mode I, II, and I/II fracture in 5-HS carbon/epoxy cloth with identical weft and fill yarns. The authors noted that the microstructure in the interface region containing a crack had an effect on both the rate and uniformity of crack propagation 
and the value of fracture toughness along the length of the beam. Non-linearity was observed in the load-displacement data directly before each crack growth, and high toughness was observed every $12 \mathrm{~mm}$, correlating to the size of the unit cell, indicating a further microstructure dependency for fracture toughness. A great deal of transverse yarn debonding was observed in this study, consuming excess energy thus leading to higher than anticipated fracture toughness values. Transverse yarn debonding, as seen in Figure 1.8, occurs when a yarn perpendicular to the delamination direction is not held down by a fill or weft yarn and is allowed to separate from the ply. These "free fill" or "free weft" yarns consume excess energy and are very common in most WFCs. Interestingly, fiber bridging was not seen to be a major contribution to fracture work in WFCs, unlike its role in unidirectional laminates, where it is the main cause of undesired consumption of strain energy leading to higher fracture toughness values. $G_{I c}$ was found to be much higher in WFCs than UDCs. Run-arrest crack growth was recorded and attributed to the debonding of transverse yarns.

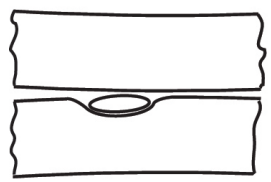

(i)

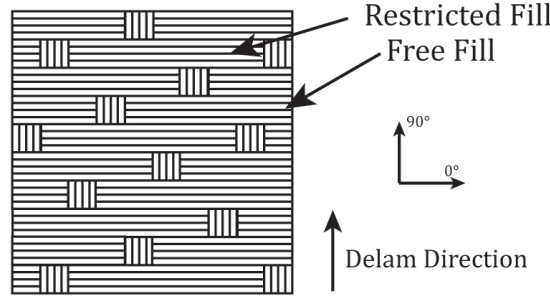

(ii)

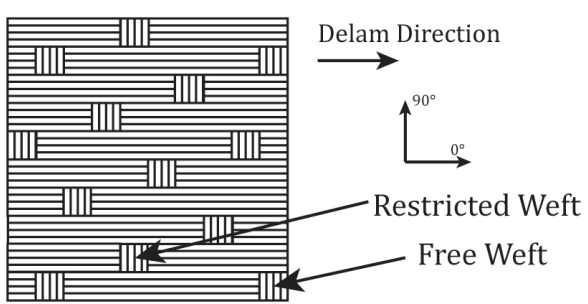

Figure 1.8: (i): Transverse yarn debonding (ii): Free fill and weft yarns

In 1998, Alif et al. performed another study on mode I fracture behavior in woven fabric composites [14]. The crack propagation direction was varied between the fill and weft yarns of plain, twill, and 8-HS glass/epoxy laminates and a 5-HS carbon/epoxy laminate. Fiber bridging was observed in the 8-HS weave and was attributed to the high amount of allowable free weft/fill yarns at the edges. These free yarns were easily debonded from their original plies and caused an increase in the calculated fracture toughness. The 5-HS weave did not experience any form of fiber bridging, but transverse yarn debonding was observed. A more 
stable crack growth occurred during this series of testing and was attributed to the manner in which the laminates were created. The authors suggested that a mirror symmetry in the delamination interface can cause unstable run-arrest crack growth, as is usually seen in WFCs. Mirror symmetry of the delamination interface does not refer to the symmetry of the laminate but, rather, to the matching up of the fill and weft yarns above and below the delamination plane. Fracture toughness was found to increase with increasing weave index and reached a steady state (non crack length dependent) value for the twill and harness satin composites after several initial propagations. The plain weave composite did not appear to be crack length dependent for any value recorded. Crack propagation along the fill yarns occurred with lower energy input than along the weft yarns, supporting the conclusion of anisotropy in steady state values of $G_{I c}$ for the twill and harness-satin weave patterns.

Suppakul and Bandyopadhyay [65] studied mode I fracture in plain, twill, 4-HS and 8HS E-glass fabric with a rubber-modified vinyl ester resin. Similar to the studies discussed above, fiber bridging was not observed in the plain weave composite. It was a factor in the other three weaves, however, which would not be expected after reviewing the studies done by Alif et al. where only the higher 8-HS cloth exhibited fiber bridging. Transverse yarn debonding also had a large influence on the measured fracture toughness of the twill and harness-satin weaves. Great care was taken in this study to ensure mirror symmetry in the delamination interface. Fracture toughness was noted to increase monotonically for each weave initially, followed by a stabilization of $G_{I c}$ with further propagation. The harnesssatin weaves were observed to have the most stable values for $G_{I c}$ after a crack length of $a=20 \mathrm{~mm}$ whereas the other weave pattern had further fluctuation after the same crack length. Generally, fracture toughness was found to increase with increasing weave index, which is in agreement with the previous studies.

\subsubsection{Data Reduction Methods for the Double Cantilever Beam Test}

There are two main forms of toughness calculated during DCB tests: propagation, and initiation values. The initiation value, as the name suggests, is calculated from the first propagation past the delamination insert. The propagation values are any other recorded 
crack progressions other than initiation. These are, in general, strongly dependent on fiber bridging, transverse yarn debonding, and the other imperfections mentioned in $\S 1.2 .1$. Initiation values are often preferred for this reason but care must be taken when inserting the initial delamination to decrease its effect on stress concentrations. The ASTM standard D5528 contains a compromise between using either the initiation or propagation values by requiring unloading of the specimen after $3-5 \mathrm{~mm}$ of crack propagation past the insert. A reloading cycle to continue crack growth allows for the calculations of $G_{I c}$ from initiation and propagation.

DCB specimens normally have a starter film inserted within the laminate during the layup process. The thickness and material of the film both have a high influence on its performance. A material that reacts with the resin used in the laminate (i.e. a material that will bond to the resin) will require an excess energy input for a crack propagation, and using a release agent to treat the film may change the resin characteristics. Teflon is normally recommended for film material because it does not require an release agent application to prevent resin adherence for most resin systems. Starter film thickness was investigated in round robin testing where it was found that $G_{I c}$ decreased with decreasing film thickness down to a thickness of $13 \mu \mathrm{m}$ (0.0005in) where $G_{I c}$ stabilized [6, 28-30, 35, 48, 55].

The question of when failure actually occurs must also be considered; three definitions of crack initiation are commonly used. The visual onset (VIS) value is obtained by using the load and displacement readings corresponding to a visual propagation of the crack front at the edge of the DCB specimen. This value often yields a high estimation of $G_{I c}$ because damage often occurs before visual onset is observed (i.e. damage at the interior of the specimen). A change in system compliance can also suggest initiation (5\%/Max). A $5 \%$ increase in compliance often corresponds to $1 \mathrm{~mm}$ of crack extension past a $50 \mathrm{~mm}$ delamination insert [27]. If the $5 \%$ increase in compliance cuts through the load-displacement plot before the maximum load to cause crack growth, then the value of load and displacement corresponding to the intersection of the $5 \%$ compliance increase and the load-displacement curve should be used. If the intersection is beyond the maximum load point, then this initiation 
value should not be recorded. The final, and most popular, initiation value is the point at which the load-displacement curve becomes non-linear (NL). A typical load-displacement curve is illustrated in Figure 1.9 with each of the initiation values indicated. The fracture resistance curve (R-curve) on the right indicates the spread in toughness values predicted by each method.

In 1987, Smiley and Pipes [63] suggested a method for calculating fracture toughness based on the area underneath the load-displacement curve and the difference between crack lengths. The main problem with this method is that toughness is based on the difference between crack lengths so it is not calculated at each recorded crack, but between each crack. Since an additional point after initiation is required, initiation values cannot be recorded and R-curves cannot be created. ASTM D5528 does not recommend the use of the area method for these reasons. The most commonly used methods for the calculation of $G_{I c}$ are the Modified Beam Theory (MBT) proposed by Hashemi et al. [34], the Compliance Calibration Method (CC) proposed by Berry [19], and the Modified Compliance Calibration Method (MCC) proposed by Kageyama and Hojo [42]. The MBT is recommended as the main data reduction method because it yields the most conservative values. The three methods, above, were within $3.1 \%$ of each other during round robin testing performed by O'Brian and Martin [55]. The MBT, CC, and MCC methods will be discussed in depth in Chapter 2.

In 1985, Keary et al. evaluated the applicability of three methods for the calculation of mode I fracture toughness in unidirectional composites [45]. They used an energy rate determination of the J-integral (method 1), the CC method (method 2), and another equation based on linear beam bending (method 3$)$. The authors pointed out that the assumptions inherent in specific testing environments and material behavior will affect the choice of which data reduction method to use. These assumptions are nonlinear beam bending behavior, usually coinciding with large deflections; a difference between the tensile and flexural moduli used in most linear elastic fracture mechanics (LEFM) calculations; neglection of the shear component of beam deflection; encastré beam support conditions at the crack tip; nonlinear material behavior; the effect of non-isotropy on beam dimensions; laminate 


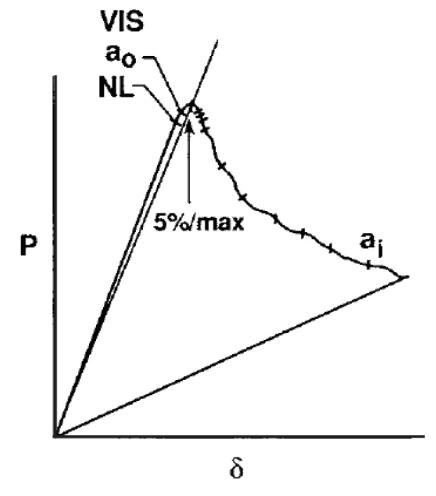

(i)

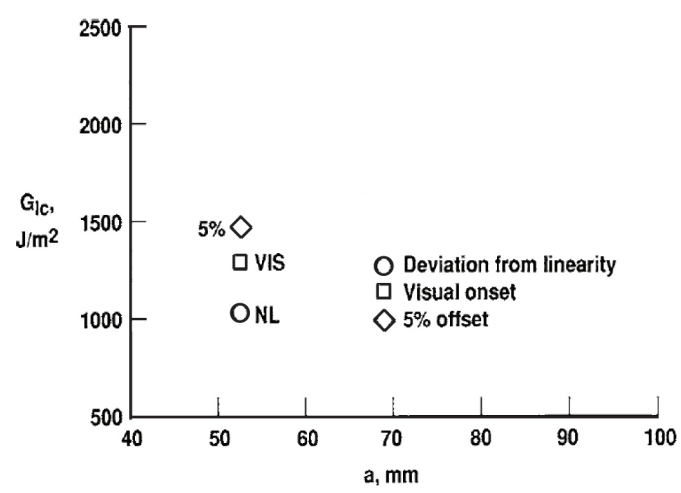

(ii)

Figure 1.9: (i): Typical load-displacement $(P-\delta)$ curve (ii): Corresponding fracture resistance spread $[6]$

reaction to applied loads; and plastic deformation. Assumptions for the J-integral did not put any restrictions on material type or specimen behavior and it was expected to be the most general approach. This method produced the lowest values of $G_{I c}$, while the highest values were calculated using the equation based on linear beam bending which assumed specific specimen and material behavior. Method 3 assumed a linear-elastic, homogeneous material with perfect cantilever beam behavior and no shear deflection. The validity of this approach was questioned after calculating the corresponding tensile modulus of the material for each crack length. Method 3 forced the tensile modulus to change with changing crack lengths, which violated the true material behavior. The CC method assumed linear behavior in the $P-\delta$ curve for all crack lengths and was found to slightly overestimate $G_{I c}$. Overestimate aside, this method is a good relative measure of fracture toughness, while the J-integral method works best if nonlinear behavior is evident. Much like the area method, the J-integral depends on differences between crack lengths and does not calculate toughness at each propagation, eliminating the chance to obtain an initiation value and R-curves.

Marom et al. [47] performed testing on E-glass, Carbon, and Kevlar WFCs in 1988 and used a calculation based on Berry's compliance calibration method [19]. Whereas the ASTM version of CC (see Chapter 2 for a mathematical description) uses the slope of the line 
formed by plotting the log of system compliance on the abscissa and the log of crack length on the ordinate, Marom et al. incorporated a form which includes the intersection of that line on the abscissa. This method has the advantage of being able to calculate toughness at every point on the $P-\delta$ curve but these values have no meaning if a crack growth did not occur. The values predicted by this method are higher than the version presented by ASTM D5528 which are already slight over-predictions of toughness, according to Keary et al. [45].

Bolotin et al. [20] postulated that, if fracture toughness in isotropic materials is characterized by a scalar, then $G_{I c}$ for a material which breaks from isotropy should be described by a tensor, specifically, a second order tensor for materials with two in-plane principal material directions. Consequently, the fracture toughness will change with every in-plane change in delamination direction. Mode I testing of unidirectional Aramid/Epoxy and woven Glass/Epoxy laminates with varying crack growth directions produced data which correlated well with the predictions from this method [20]. Knowing that fracture toughness will be equal in both principal material directions for laminates with cross-ply symmetry (i.e. plain and basket weaves) as proven by $[15,37,59]$, the tensor should be able to predict non-direction-dependent fracture toughness values in all crack growth directions. The results of these tests, however, did not match the prediction. Crack propagations between $0^{\circ}$ and $30^{\circ}$ had relatively constant $G_{I c}$ but a significant decrease occurred after an angle of $45^{\circ}$ [37]. After further testing on unidirectional laminates, the tensor was proven effective in determining fracture toughness dependence on delamination growth direction in a interface region composed only of unidirectional laminae.

In 1985, Aliyu and Daniel [16] studied the strain rate dependence of $G_{I c}$ in Graphite/Epoxy unidirectional laminates. Their data reduction incorporated the popular beam analysis method described in [69] which treats the DCB specimen as two perfect cantilevered beams. This method assumes that all of the strain energy resulting from the opening of the crack is stored in the open part of the beam, which would only be true if the uncracked portion of the beam were perfectly rigid. The authors noted that at least some of the strain energy would actually be stored in the uncracked portion of the beam and mentioned an alternate 
form of the beam analysis, presented by $[43,56,61]$. This method changes the encastré boundary condition at the crack tip to that of a beam supported on an elastic foundation over the length of the uncracked region. Another assumption commonly used is that shear stress does not play a significant role in the deformation of the beam. The anisotropy of some composite systems, though, can amplify the shear effects until they can no longer be ignored. Aliyu and Daniel modified the expressions for system compliance and strain energy release rate to account for the shear effect, making them functions of the shear modulus of rigidity as well. They also had to account for the effects of the high displacement rates used in their testing. The expression for strain energy release rate was further modified to include kinetic energy, making it a function of crack velocity and longitudinal wave propagation velocity. They found that the strain energy release rate increased by $28 \%$ over the range of displacement rates tested and were able to express $G_{I c}$ as a power law of crack velocity for all displacement rates.

\subsubsection{Post-Bond Delamination Resistance}

Few studies have been conducted concerning the relative strength of a post-bonded laminate to a traditional laminate. A post-bond, as described in $\S 1.1$, differs from an adhesive bond (secondary-bond) in that the adhesive bond implies a joining together of fully cured laminates, while the post-bond implies that one of the laminates is fully cured and the other cures on top of it. Adhesively bonded joints have been studied considerably [23, 26, 46, 49] and there are several ASTM standards relating directly to their testing [9-12]. Secondary bonds, post-bonds, and co-cures are all active research topics but finding any published experiments regarding mode I fracture of post-bonded composites has proven to be a difficult task. Many papers suggest that a main reason for some composite structural failures is the use of secondary bonds or post-bonds but the testing done was on traditional (co-cured) DCB specimens. The failure mode of secondary and post bonds can be cohesive (the adhesive sticks to both sides of the laminate but cannot hold them together), adhesive failure (the adhesive does not stick to one side of the laminate), or a mixture of both. 
Campilho et al. [23] performed mode I fracture testing on co-cured and secondarybonded natural fiber composites. $G_{I c}$ for the co-cured specimens was about $2 \mathrm{~N} / \mathrm{mm}$ and $1.43 \mathrm{~N} / \mathrm{mm}$ for the secondary bonded specimens. The initiation value of the co-cured specimens was $G_{I c}=0.9 \mathrm{~N} / \mathrm{mm}$ which is lower than the value of $G_{I c}=1.2 \mathrm{~N} / \mathrm{mm}$ obtained for the secondary bond. The authors speculated that the reason for the co-cured plate having a higher steady state value of $G_{I c}$ was that fiber bridging began almost immediately after the initial crack and continued for the remainder of the test. In 1986, Mall and Johnson [46] performed testing on secondary bonds of Graphite/Epoxy laminates with two adhesive systems: EC 3445, a thermosetting paste, and FM-300, an epoxy adhesive contained within a cloth carrier. The EC 3445 bond was made purely by bonding the two fully cured laminates together. The FM-300 bond was created in a co-cure manner because of the carrier cloth. The cloth was inserted in the middle of the laminate and cured along with the Graphite/Epoxy. Mall and Johnson found that the EC 3445 laminate under pure mode I loading failed in a cohesive manner but the FM-300 laminate failed cohesively, adhesively, and both in different areas along the length of the specimen. The authors determined that toughness in adhesive failure is $40 \%$ lower than in cohesive failure. Based on these findings, it is reasonable to assume that post-bonded laminates will behave similarly to the FM-300 specimens above and fail in an irregular manner with cohesive, adhesive, and mixed failure along the length of the beam.

\subsubsection{Summary of Literature Review Topics}

Several main conclusions can be drawn from the literature review, above. Chief among these conclusions is the effect of specific energy dissipation terms on the fracture toughness results. Recall that energy dissipation can take several forms viz. crack jumping, fiber pull-out, fiber breakage, fiber bridging, matrix deformation, matrix cracking, crack branching, crack deviation, and transverse yarn debonding. In general, the effects of these terms are minimized at the insert, meaning the most consistent values of fracture toughness will be determined from the insert initiation. Past this point, fracture toughness gradually approaches a steady state value as the energy dissipation effects reach their steady state. This difference between the initiation and steady state $G_{I c}$ value is an important consideration 
in the comparison of delaminations propagating in different directions. $G_{I c}$ isotropy was witnessed more often when the comparison was made between initiation, rather than the steady state, values. Anisotropy between $G_{I c}$ for differing delamination directions was determined when steady state values were considered, allowing for energy dissipation to effect fracture toughness. Energy dissipation effects could be mitigated through the careful use of edge delaminations, or through the use of a ductile, rather than a brittle, matrix.

Woven fabric composites have a higher risk of out of plane crack propagation and transverse yarn debonding than unidirectional composites. Their complex weave structure tends to complicate the fracture and raise the risk of run-arrest crack propagation which can artificially increase the fracture toughness. Simpler, homogeneous fabrics like plain and basket weaves tend to have a lower discrepancy between their initiation and steady state fracture toughness values than the more complicated twill or harness-satin weaves. The orientation of the delamination interface plies is a major factor in the steady state value of fracture toughness. It has been suggested that composite delamination be treated as a structural, rather than a material property, to alleviate the effect of larger energy dissipation effects for off-axis delaminations which would imply a more phenomenological definition of a composites' resistance to crack growth.

The choice of which data reduction method to incorporate in a study is case dependent. Kinetic energy terms can be used to adjust for high load application rates, alternate boundary conditions can be employed to adjust for end rotation of the DCB specimen, and shear deformation can be considered a contributing factor. Alternate formulations of fracture toughness may also be used such as the J-integral approach, the Area method, and Bolotin's fracture isotropy tensor. The choice depends on the assumptions made in the formulation of each method. The system being tested must conform to the assumptions made in order to produce the most accurate results. The suggested methods by ASTM D5528 are the MBT, CC, and MCC methods which produce $G_{I c}$ values within $3.1 \%$ of each other. 


\subsection{Specific Research}

The purpose of the study herein is to analyze delamination (Mode I) in several laminate configurations of eight-harness-satin carbon cloth. The effect of post-bonded joints will be examined through the evaluation of a patent pending delamination insertion method. ASTM D5528 will be followed closely where applicable. Deviations will be made, as necessary, to account for the use of WFCs rather than UDCs. Delamination direction-dependence

will be considered only for the two principal material directions and an attempt will be made to use composite theory within the linear elastic fracture mechanics calculations for $G_{I c}$. 


\section{Chapter2}

\section{Mode I Composite Fracture Analysis}

\subsection{Introduction}

Mode I fracture in composites is generally considered the most important form of delamination to study. The double cantilever beam test has become the most commonly used test for the characterization of mode I fracture, as mentioned in Chapter 1, and is the only test suggested by ASTM D5528. The data reduction methods discussed in this chapter are all based on the DCB specimen. The accurate measurement of fracture toughness is dependent on the method used to interpret how energy is transfered during delamination. Several linear elastic fracture mechanics (LEFM) methods have been documented which attempt to accurately model the complex energy transfer, as discussed in Chapter 1. Four common data reduction methods will be discussed here, including the Area Method, the Modified Beam Theory (MBT), the Compliance Calibration Method (CC), and the Modified Compliance Calibration Method (MCC). An additional modification will be introduced to calculate an equivalent beam stiffness based on composite laminate theory and the assumptions inherent in beam calculations. These five methods should produce similar results as long as the relationship between load and displacement at the point of load application remains linear.

The DCB specimen, see Figure 2.1, consists of two identical cantilever beams with encastré boundary conditions at the crack tip, width, $b$, and lengths equal to the crack length, $a$. The total thickness of the specimen is $h$, meaning the individual beams each

have thickness $\frac{1}{2} h$. The deflection measured during testing is the cross head displacement, 
$\delta$, but the deflection of each beam is $\frac{1}{2} \delta$, caused by the vertical force, $P$. Values for $P, \delta$, and $a$ will change throughout the testing procedure while $b$, and $h$ will remain constant. Values of $G_{I c}$ can be calculated for every recorded value of crack length with the MBT, CC, MCC, and the equivalent beam stiffness (EQS) methods. The area method, on the other hand, only allows for calculations between crack lengths, as will be discussed in $\S 2.2$.
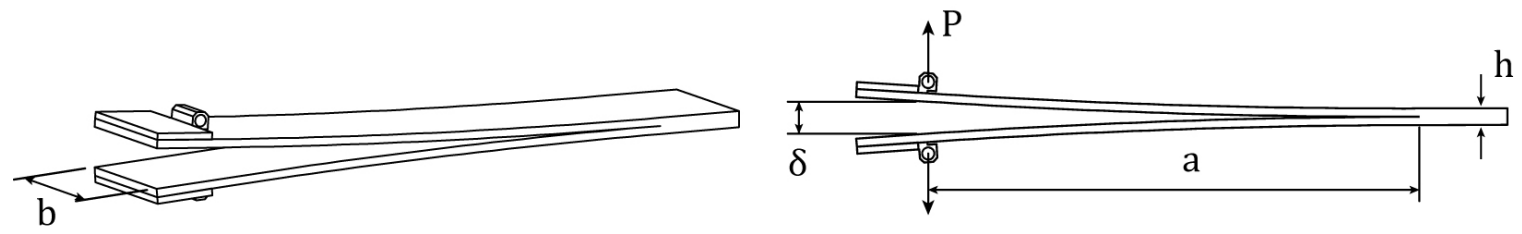

Figure 2.1: DCB test specimen dimensions

Fracture in solids, isotropic or anisotropic, is normally initiated by some defect in the material which causes a high stress concentration and localized failure of the material [2]. Griffith [33] proposed a criteria based on the $1^{\text {st }}$ law of thermodynamics which stated that the decrease in strain energy due to a propagation of a crack is used to create new crack surfaces in isotropic materials. The strain energy release rate, $G_{I}$, is a measure of the amount of strain energy released for an infinitesimal change in crack surface area, making $G_{I}$ a rate with respect to area, rather than time. Fracture toughness, $G_{I c}$, is the critical value of the strain energy release rate which causes an extension of the crack [2]. The use of LEFM is widely accepted as an appropriate tool for the characterization of fracture in metal structures. Sih and Liebowitz [62], extended these concepts to orthotropic materials. Recall: an orthotropic material is one in which changes in lengths and internal angles of a unit of material will occur with the application of a normal or shear stress in any direction excluding the principal material directions. Application of normal stresses in the principal directions will only produce changes in length. Likewise, shear stresses applied in the principal material directions will only produce changes in internal angles. 


\subsection{Area Method}

The strain energy release rate, as stated above, is a measure of the amount of strain energy released for an infinitesimal change in crack surface area. If $U$ is the strain energy and $A$ is the crack surface area, $G_{I}$ can be written in mathematical form as

$$
G_{I}=-\frac{d U}{d A} .
$$

The crack surface area is equal to the length of the crack multiplied by the width of the beam, $A=a b$. Knowing that $b$ is a constant, the expression can be rewritten as

$$
G_{I}=-\frac{1}{b} \frac{d U}{d a}
$$

which is the most common LEFM expression of $G_{I}$ for DCB testing.

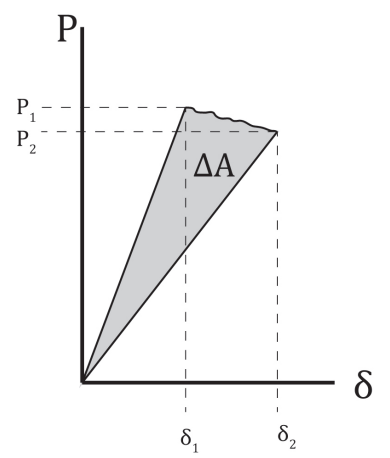

Figure 2.2: $P-\delta$ trace for area method calculations

Consider the example $P-\delta$ curve in Figure 2.2. Crack propagations are labeled on the ordinate and the abscissa with their corresponding displacements and loads. The total strain energy released due to a crack length change from $a$ to $a+d a$ would be the area between the loading and unloading lines. If the $P-\delta$ curve between loading and unloading 
can be approximated by a straight line, the change in energy, $\Delta U$ can be expressed as

$$
-\Delta U=\Delta A=\frac{1}{2}\left(P_{1} \delta_{2}-P_{2} \delta_{1}\right)
$$

Combining equations 2.2 and 2.3 yields a final expression for fracture toughness for the area method:

$$
G_{I c}=\frac{1}{2 b \Delta a}\left(P_{1} \delta_{2}-P_{2} \delta_{1}\right)
$$

Equation 2.4, above, calculates a value for $G_{I c}$ corresponding to a change in crack length, not an instantaneous crack length. The area method will not be used in this study because it cannot attain $G_{I c}$ for crack initiation.

\subsection{Modified Beam Theory}

Linear elastic fracture mechanics was originally developed for the analysis of throughthickness cracks in homogeneous, isotropic materials. Stress analysis formulations of fracture toughness have been made for many loading conditions and crack geometries $[40,67]$ in isotropic materials but anisotropic materials present many more mathematical difficulties [32]. The strain energy release rate method was formulated to present an alternate way of finding fracture toughness which works for both isotropic and anisotropic materials. The following steps were modified from Gibson's [32] interpretation of Irwin's [41] original derivation. The final differential expression for the strain energy release rate in terms of DCB specimen dimensions and system compliance will be used in the MBT to formulate an expression without partial derivatives.

\subsubsection{General Form of the Strain Energy Release Rate}

Consider the homogeneous, isotropic block of material with a through thickness crack in Figure 2.3. The strain energy release rate is defined as the rate of change in strain energy with respect to crack surface area, $A$. The strain energy can be calculated by finding the difference between the incremental work done, $\Delta F$, and the potential energy released, $\Delta \Pi$, 


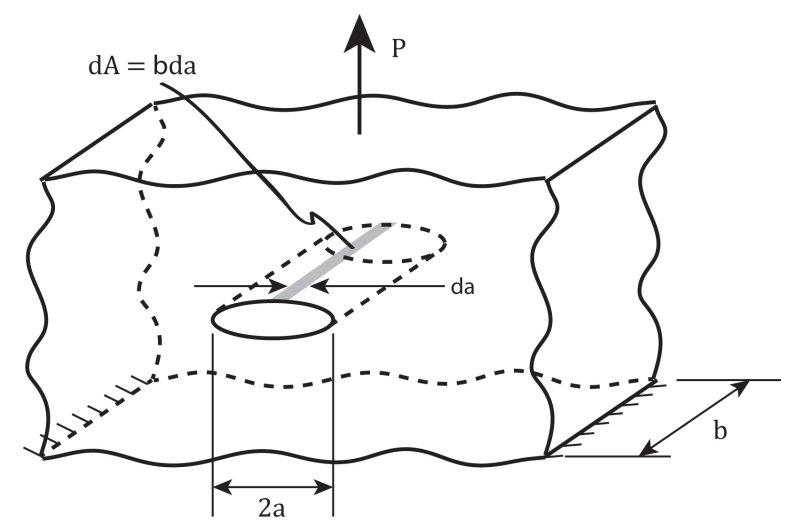

Figure 2.3: Griffith Crack: A through-thickness crack in a uniaxially loaded plate of infinite width

during crack extension. The expression for the strain energy released is as follows:

$$
\Delta U=\Delta F-\Delta \Pi
$$

The expression for the strain energy release rate is

$$
\begin{aligned}
G_{I} & =\frac{\partial U}{\partial A} \\
& =\lim _{\Delta A \rightarrow 0} \frac{\Delta U}{\Delta A} \\
& =\lim _{\Delta A \rightarrow 0} \frac{\Delta F-\Delta \Pi}{\Delta A} \\
& =\frac{\partial F}{\partial A}-\frac{\partial \Pi}{\partial A} .
\end{aligned}
$$

The incremental work and the original potential energy can be described through simple expressions, assuming linear relationships between load and displacement as displayed in Figure 2.4.

$$
\begin{gathered}
\Pi=\frac{1}{2} P \delta, \\
\Delta F=P \Delta \delta .
\end{gathered}
$$




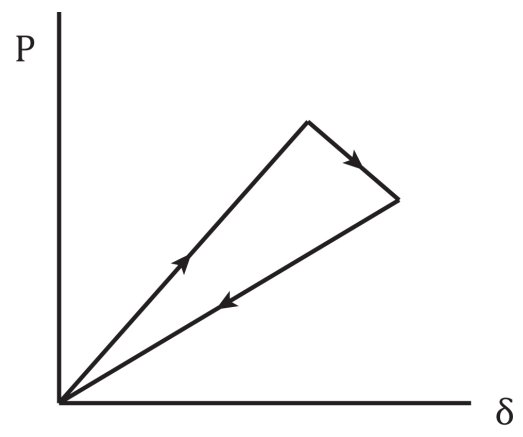

Figure 2.4: LEFM example $P-\delta$ plot [32]

Knowing that the system compliance is equal ot the displacement divided by the load:

$$
C=\frac{\delta}{P}
$$

the derivatives in Equation 2.6 can be evaluated:

$$
\begin{aligned}
\frac{\partial \Pi}{\partial A} & =\frac{\partial}{\partial A}\left(\frac{1}{2} C P^{2}\right) \\
& =C P \frac{\partial P}{\partial A}+\frac{1}{2} P^{2} \frac{\partial C}{\partial A},
\end{aligned}
$$

and

$$
\begin{aligned}
\frac{\partial F}{\partial A} & =\lim _{\Delta A \rightarrow 0} \frac{\Delta F}{\Delta A} \\
& =\lim _{\Delta A \rightarrow 0} P \frac{\Delta \delta}{\Delta A} \\
& =P \frac{\partial \delta}{\partial A} \\
& =P \frac{\partial(C P)}{\partial A} \\
& =P^{2} \frac{\partial C}{\partial A}+C P \frac{\partial P}{\partial A} .
\end{aligned}
$$


Substitution of Equations 2.10 and 2.11 into Equation 2.6 yields the reduced form of the linear elastic fracture mechanics (LEFM) definition of the strain energy release rate.

$$
\begin{aligned}
G_{I} & =P^{2} \frac{\partial C}{\partial A}+C P \frac{\partial P}{\partial A}-C P \frac{\partial P}{\partial A}-\frac{1}{2} P^{2} \frac{\partial C}{\partial A} \\
& =\frac{1}{2} P^{2} \frac{\partial C}{\partial A} .
\end{aligned}
$$

Noting that the infinitesimal crack surface area, $d A$, is equal to the product of the block width and the infinitesimal change in crack length, $d a$ :

$$
G_{I}=\frac{P^{2}}{2 b} \frac{\partial C}{\partial a}
$$

\subsubsection{Modified Beam Theory}

The major assumptions of the MBT are the same as the assumptions made for the flexure of straight and curved beams. The assumptions for the following analysis are that the beam is made of a homogeneous material with the same modulus of elasticity in tension and compression; the beam is straight, defined by a possible radius of curvature at least ten times larger than the thickness of the beam in the plane of bending; the beam has a uniform cross section; there is at least one longitudinal axis of symmetry; all loads and reactions act perpendicular to the axis of the beam and lie in the longitudinal axis of symmetry; the length of the beam is at least 8 times larger than the thickness; the beam is not disproportionately wide; and the maximum stress does not exceed the proportional limit [57].
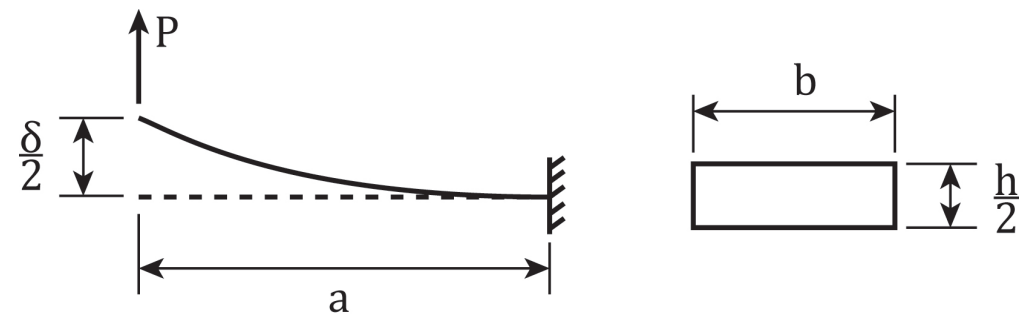

Figure 2.5: Basic beam diagram for modified beam theory calculations 
The equation defining deflection of a cantilever beam like the one in Figure 2.5 is

$$
\frac{1}{2} \delta=\frac{P a^{3}}{3 E I}
$$

where $E$ is the modulus of elasticity of the beam corresponding to the longitudinal axis and $I$ is the moment of inertia of the beam section with respect to the neutral axis. The moment of inertia for the beam in Figure 2.5 is

$$
\begin{aligned}
I & =\frac{1}{12} b\left(\frac{1}{2} h\right)^{3} \\
& =\frac{1}{96} b h^{3} .
\end{aligned}
$$

Substituting Equation 2.15 into 2.14 and rearranging:

$$
\delta=\frac{64 P a^{3}}{E b h^{3}}
$$

The application of this expression to LEFM is achieved through the use of Equation 2.13 which requires the partial derivative of system compliance with respect to crack length. The compliance, $C$, is the deflection, $\delta$, divided by the applied load, $P$, so

$$
\begin{aligned}
C & =\frac{1}{P} \frac{64 P a^{3}}{E b h^{3}}, \\
\frac{\partial C}{\partial a} & =\frac{3\left(64 P a^{2}\right)}{P E b h^{3}} \\
& =\frac{3}{P a} \frac{64 P a^{3}}{E b h^{3}} \\
& =\frac{3}{P a} \delta .
\end{aligned}
$$

Substitution of Equation 2.18 into 2.13 yields the expression

$$
G_{I}=\frac{3 P \delta}{2 b a}
$$

In 1974, Kanninen [44] observed that the forces and moments on an isotropic DCB specimen cause the end of the beam to deform, which implies that the assumption of encastré boundary conditions at the crack tip is incorrect. The rotation of the beam can 
be accounted for by adjusting the value of crack length used in the calculation. A value, $\Delta$, should be added to the measured crack length, $a$ :

$$
a_{t}=a+|\Delta|
$$

where $a_{t}$ is the value of crack length that should be used in Equation 2.19. The adjustment, $\Delta$, can be found by creating a least squares plot of the cubed root of compliance, $C^{\frac{1}{3}}$, with respect to the measured crack length, $a$. The values used in the calculation of compliance should be the load and deflection corresponding to visual observation (vis) of the crack rather than the NL or (5\%/Max) values. The resulting plot, see Figure 2.6, should produce a line which, if extended, will intersect the ordinate at the value $\Delta$. If the line passes through the origin, no correction term is needed, i.e. $\Delta=0$. The final expression for the strain energy release rate is

$$
G_{I}=\frac{3 P \delta}{2 b(a+|\Delta|)} .
$$

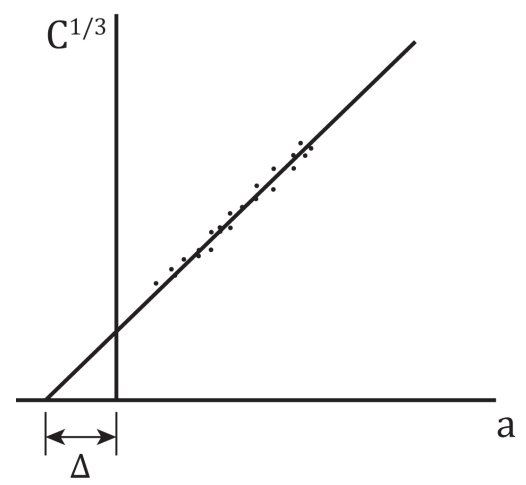

Figure 2.6: MBT crack length correction term

Round-robin testing aimed at evaluating the applicability of the MBT, CC, and MCC methods was performed in the 1992 [55]. The values of $G_{I c}$ calculated using each method did not vary by more than $3.1 \%$, and the MBT proved to be the most conservative method, producing the lowest values of $G_{I c}$ for $80 \%$ of the specimens [6]. 


\subsection{Compliance Calibration Method}

The compliance calibration method, originally proposed by Berry in 1963 [19], begins with a slight reorganization of Equation 2.14, where

$$
P=\frac{3 E I \delta}{a^{3}}
$$

This can be written in a more general form, excluding all references to material properties and specimen dimensions:

$$
P=\delta H a^{-n}
$$

where $H$ and $n$ are experimentally determined coefficients. A more convenient form of this equation would require only one of these values, eliminating $H$, for instance. This can be done by returning to Equation 2.2 , the definition of $G_{I}$ :

$$
G_{I}=-\frac{1}{b} \frac{d U}{d a}
$$

where $\mathrm{U}$ is defined in Equation ??:

$$
U=\frac{1}{2} P \delta
$$

Combining Equations ?? and 2.22 allows the strain energy to be written in terms of $H$ and $n:$

$$
U=\frac{1}{2} \delta^{2} H a^{-n}
$$

The application of Equation 2.2 yields:

$$
\begin{aligned}
G_{I}= & -\frac{1}{2 b} \delta^{2} H(-n) a^{-(n+1)} \\
= & \frac{\delta H}{a^{n}} \frac{n \delta}{2 b a} \\
& G_{I}=\frac{n P \delta}{2 b a} .
\end{aligned}
$$

The CC method is widely used for the calculation of fracture toughness in composite DCB specimens $[14,15,19,45-47,63,69]$ and has the benefit of not requiring any material 
properties. The applied load, $P$, and the deflection, $\delta$, corresponding to each crack length, $a$, along with the beam width, $b$, are all known quantities. The experimentally determined coefficient, $n$, is the only value that is unknown until the end of the testing series. It can be found by returning to Equation 2.22 and rewriting it as:

$$
C=\frac{a^{n}}{H}
$$

Changing Equation 2.26 it to its logarithmic form:

$$
\log (C)=n \log (a)-\log (H)
$$

This shows that there is a linear relationship between the logarithms of system compliance and crack length. The slope of the line formed when $\log (C)$ is plotted on the abscissa and $\log (a)$ on the ordinate will be $n$, as shown in Figure 2.7.

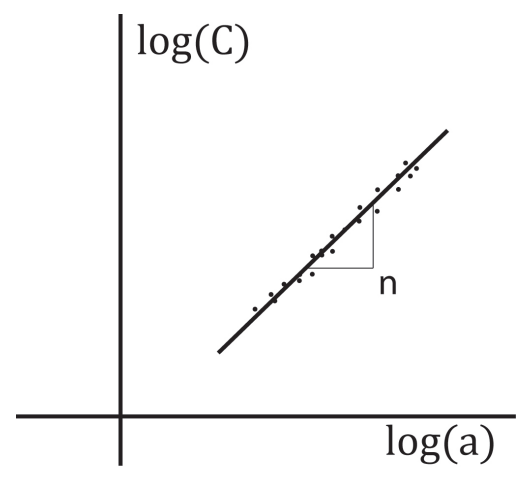

Figure 2.7: Compliance calibration experimentally determined coefficient

\subsection{Modified Compliance Calibration Method}

The MCC method, proposed by Kageyama [42], much like the CC method, begins with Equation 2.14, the deflection expression for the DCB specimen:

$$
\frac{1}{2} \delta=\frac{P a^{3}}{3 E I}
$$


Following the steps taken in $\S 2.3 .2$ to obtain the expression for the compliance of the DCB specimen,

$$
C=\frac{64 a^{3}}{E b h^{3}}
$$

Rearranging the compliance:

$$
\frac{E b}{64} C=\left(\frac{a}{h}\right)^{3}
$$

Equation 2.28 can be written in a more general form, as was done in $\S 2.4$ to exclude material properties:

$$
A_{1}^{3} C=\left(\frac{a}{h}\right)^{3}
$$

so

$$
A_{1} C^{1 / 3}=\left(\frac{a}{h}\right)
$$

where $A_{1}$ is an experimentally determined coefficient.

Equation 2.30 makes it clear that the cubed root of the DCB system compliance is directly proportional to the normalized crack length $\left(\frac{a}{h}\right)$. The coefficient, $A_{1}$, can be found by plotting $\left(\frac{a}{h}\right)$ on the abscissa and $C^{\frac{1}{3}}$ on the ordinate. The slope of the corresponding least-squares fit is $A_{1}$, as shown in Figure 2.8 .

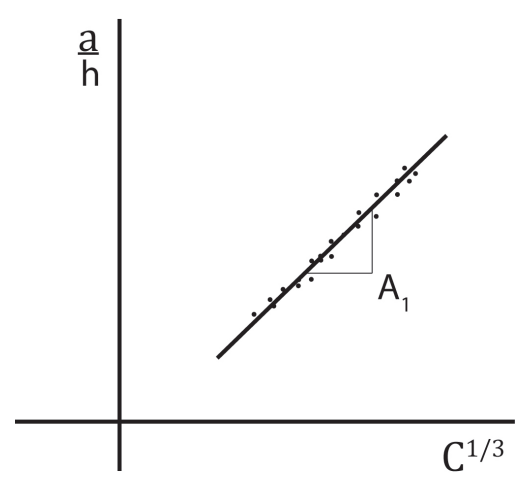

Figure 2.8: Modified compliance calibration experimentally determined coefficient 
Further manipulation of Equation 2.19 will yield the final expression of $G_{I}$ for the MCC method. Noting that

$$
\begin{gathered}
\frac{a}{h} \frac{1}{A_{1} C^{1 / 3}}=1: \\
G_{I}=\frac{3 P \delta}{2 b a} \frac{a}{h} \frac{1}{A_{1} C^{1 / 3}} \\
=\frac{3 P \delta C^{-1 / 3}}{2 b h A_{1}} .
\end{gathered}
$$

It can also be noted that

$$
\frac{P}{\delta} C=1
$$

so

$$
G_{I}=\frac{3 P \delta C^{-1 / 3}}{2 b h A_{1}} \frac{P}{\delta} C
$$

and after simplification,

$$
G_{I}=\frac{3 P^{2} C^{2 / 3}}{2 b h A_{1}}
$$

Equation 2.35 is the final expression for the strain energy release rate, as given in ASTM D5528.

\subsection{Equivalent Stiffness Method}

The equivalent stiffness method (EQS), unlike the other methods reviewed in the previous sections, has not received much, if any, attention in the composite fracture community. The only references found by this author have been in personal notes and a paper written by O'Brien in 1982 [54]. O'Brien's paper did not deal with mode I fracture testing, rather, the effects of delaminations on tensile specimens where the tensile load laid in the same plane as, and was normal to, the delamination. O'Brien was able to use the rule of mixtures and CLT (composite laminate theory) to form an expression for the reduced damage modulus in symmetric laminates.

The EQS method uses CLT to derive an equivalent stiffness term for a doubly-symmetric DCB specimen which conforms to the assumptions in $\S 2.3 .2$ and does not restrict anticlastic 
bending. Note that the term "doubly symmetric" means that the laminate must be symmetric about the delamination plane and each cantilever beam must be symmetric about its midplane. Anticlastic bending refers to the phenomenon of induced curvature about axes other than the one to which load is applied, see Figure 2.9 for an example. The expression obtained for the equivalent stiffness will replace the EI term in Equation 2.14 and a similar approach to the derivation of Equation 2.21 will be used to formulate $G_{I}$ for the EQS method.

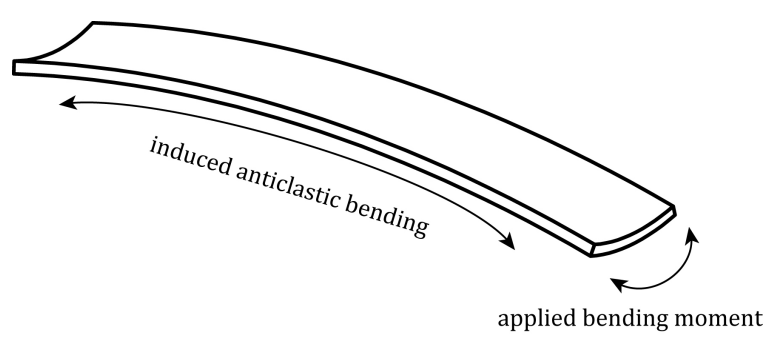

Figure 2.9: Anticlastic bending of a beam

The theory behind CLT can be reviewed in $[2,13,32,38,50]$. The resulting constitutive equations for CLT, in indicial notation, are

$$
N_{i}=A_{i m} \epsilon_{m}^{o}+B_{i q} \kappa_{q}
$$

and

$$
M_{i}=B_{i m} \epsilon_{m}^{o}+D_{i q} \kappa_{q}
$$

which relate the applied loads to the resulting midplane strains and curvatures. $N_{i}$ and $M_{i}$ are known as the line load and line moment vectors, respectively and $\epsilon_{m}^{o}$ and $\kappa_{q}$ are known as the midplane strain and curvature vectors, respectively. The matrix, $A_{i m}$ is the extension stiffness matrix and relates the line loads to midplane strains. $B_{i q}$ is called the coupling stiffness matrix and can be easily eliminated (i.e. all values equal to zero) by selecting a symmetric, balanced laminate for analysis. $D_{i q}$ is called the bending stiffness matrix and relates the line moments to plate curvatures. The selection of a doubly symmetric DCB 
specimen yields

$$
M_{i}=D_{i q} \kappa_{q}
$$

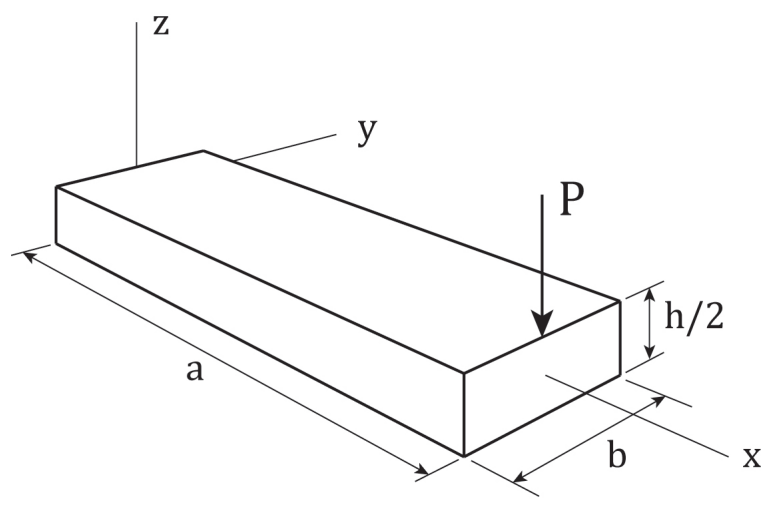

Figure 2.10: DCB beam bending specimen

For a beam like the one in Figure 2.10, with the assumptions listed above, the applied bending load can be modeled by a line moment, $M_{x}$, where the other bending moments, $M_{y}$ and $M_{x y}$, are both zero:

$$
\left\{\begin{array}{c}
M_{x} \\
0 \\
0
\end{array}\right\}=\left[\begin{array}{ccc}
D_{11} & D_{12} & D_{16} \\
D_{12} & D_{22} & D_{26} \\
D_{16} & D_{26} & D_{66}
\end{array}\right]\left\{\begin{array}{c}
\kappa_{x} \\
\kappa_{y} \\
\kappa_{x y}
\end{array}\right\} .
$$

Inverting this equation:

$$
\left\{\begin{array}{c}
\kappa_{x} \\
\kappa_{y} \\
\kappa_{x y}
\end{array}\right\}=\left[\begin{array}{lll}
d_{11} & d_{12} & d_{16} \\
d_{12} & d_{22} & d_{26} \\
d_{16} & d_{26} & d_{66}
\end{array}\right]\left\{\begin{array}{c}
M_{x} \\
0 \\
0
\end{array}\right\},
$$

so that

$$
\kappa_{x}=d_{11} M_{x}
$$


The definition of the $M_{x}$ line moment is the applied beam moment, $P a$, divided by the width of the beam, $b$. The equivalent bending stiffness, $\overline{E I}$, of the beam is the applied moment, $P a$, divided by the curvature, $\kappa_{x}$, for small angles, so

$$
\begin{aligned}
\overline{E I} & =\frac{P a}{\kappa_{x}} \\
& =\frac{b M_{x}}{d_{11} M_{x}}, \text { so } \\
\overline{E I} & =\frac{b}{d_{11}} .
\end{aligned}
$$

Equation 2.43 can now be applied to Equation 2.14 to yield

$$
\delta=\frac{2 P a^{3} d_{11}}{3 b}
$$

which can be transformed to the compliance definition:

$$
C=\frac{2 a^{3} d_{11}}{3 b}
$$

and

$$
\frac{\partial C}{\partial a}=\frac{6 a^{2} d_{11}}{3 b}
$$

Using this in the definition of $G_{I}$ from Equation 2.13:

$$
\begin{gathered}
G_{I}=\frac{P^{2}}{2 b} \frac{\partial C}{\partial a} \\
=\frac{P^{2} a^{2} d_{11}}{b^{2}}, \\
a_{t}=a+|\Delta|, \\
G_{I}=\left(\frac{P(a+|\Delta|)}{b}\right)^{2} d_{11} .
\end{gathered}
$$

The downside to the EQS method is that additional material testing must be performed before fracture toughness can be evaluated. The CLT theory requires values for the modulus of elasticity in the principal material directions, the major and minor Poisson's ratios, and 
the principal shear modulus. It also requires close attention to the layup schedule to ensure dual-symmetry about the delamination plane. The EQS method does, however, have the benefit of only requiring data collection for the load and the crack length. This may not be important for many testing environments but for those without sufficient resolution in the displacement measurement device, it could certainly be a viable option.

\subsection{Large Displacement Correction}

In 1989, Williams [70] suggested that a correction factor should be added to the calculations of fracture toughness if the deflection at the point of load application reached a limit that broke the assumption of small angles/displacement. The correction factor he derived is

$$
F_{d}=1-\frac{3}{10}\left(\frac{\delta}{a}\right)^{2}-\frac{3}{2}\left(\frac{\delta t}{a^{2}}\right)
$$

which, if at any point

$$
\frac{\delta}{a}>0.4
$$

should be used to adjust $G_{I c}$ as follows:

$$
\begin{cases}\text { if } \frac{\delta}{a}>0.4 & G_{I c}(\text { new })=F_{d} G_{I c} \\ \text { else } & \text { No Change to } G_{I c}\end{cases}
$$




\section{Chapter3}

\section{Experimental Design}

\subsection{Introduction}

Several reasons to perform material characterization for composites are to provide data needed for design and analytical purposes, inputs into finite element programs, for instance; to understand how a material will respond under specific loading conditions; and to facilitate the comparison of several candidate materials [13]. Minimum characterization generally requires $E_{1}, E_{2}, G_{12}, \nu_{12}, F_{1 t}, F_{2 t}, F_{1 c}, F_{2 c}$, and $F_{6}$ (see the Nomenclature section at the beginning of this document for definitions of these properties). The flexural modulus, $E_{f}$, is commonly desired when bending of a composite structure is likely. The short beam shear strength, $F^{s b s}$, is also commonly sought. Fracture toughness, in fact, is just another part of composite characterization. This chapter will focus on the testing preparations and procedures required for basic characterization of the specific material used in this study, excluding compressive properties. The DCB test setup will be the key focus in the final section of this chapter.

\subsection{Material}

The composite system used was a 3K 8-Harness-Satin carbon cloth weave, with identical weft and fill yarns, pre-impregnated with the Newport 307 resin system. All specimens were cured according to the manufacturer's recommendation. The cure cycle recipe can be seen in Figure 3.1, courtesy of the Newport 307 Data Sheet [52]. Great care was taken in the manufacturing of each composite plate to ensure minimum weave distortion and proper alignment of the fiber directions. Specimens for each test were cut from these plates using 


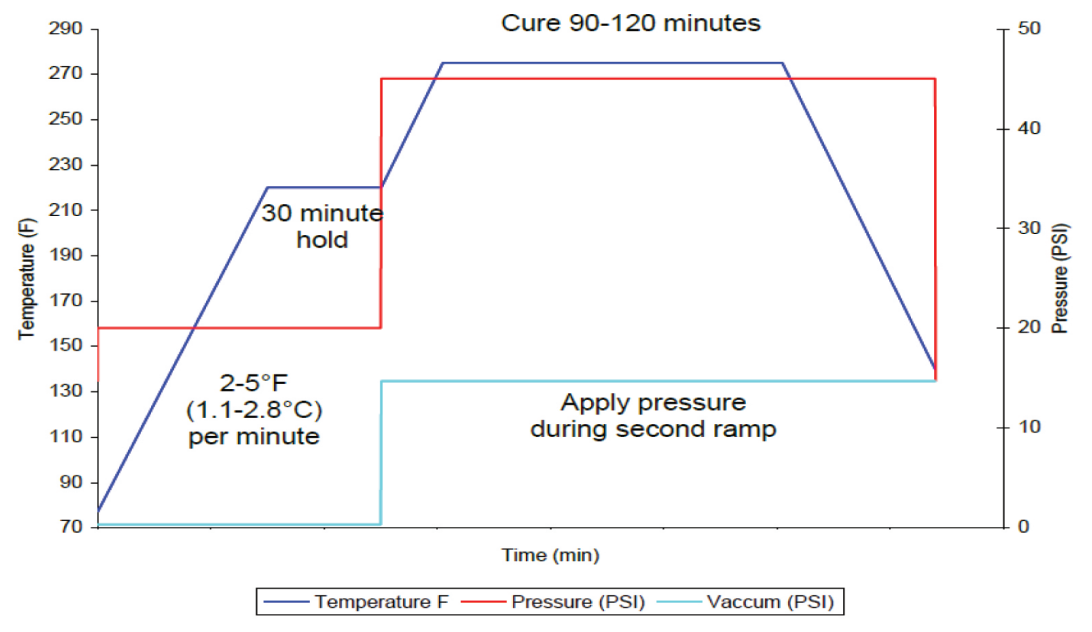

Figure 3.1: Autoclave cure cycle

a diamond-impregnated tile saw. The longitudinal and transverse directions of the fabric were defined in reference to the roll from which the cloth was taken. See Figure 3.2 for a definition of the principal material directions.

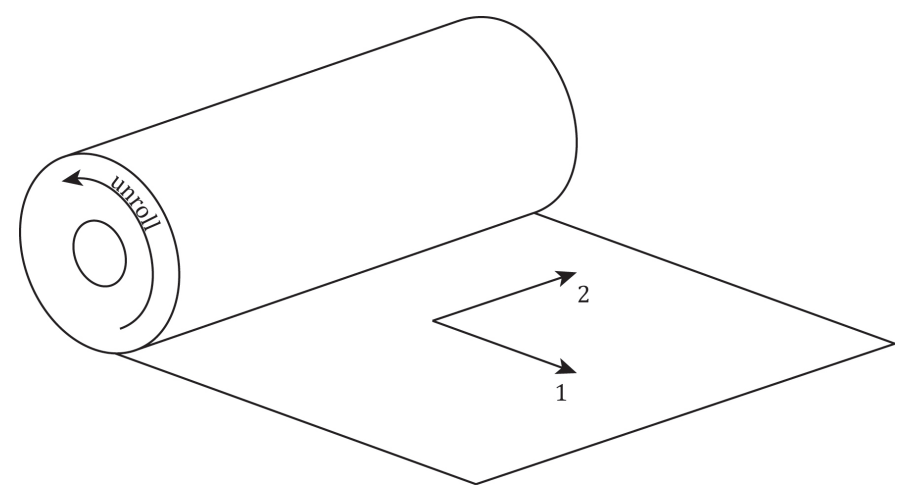

Figure 3.2: Definition of the principal material directions for this study. Note: $1 \leftrightarrow$ Longitudinal and $2 \leftrightarrow$ Transverse

Table 3.1 shows the plate numbering scheme for all of the completed laminates. Plates were numbered 1 through 13 , the specimens from each plate were numbered in a similar manner. Specimen designations consist of both the plate number and the specimen number in the format: Plate\#-Specimen\#. A coupon labeled 5-4, for instance, is the fourth specimen cut from plate five. 
Table 3.1: Plate numbering

\begin{tabular}{cl}
\hline \hline Plate Number & Purpose \\
\hline 1 & Longitudinal Pull Test \\
2 & Transverse Pull Test-Unused \\
3 & In-Plane Shear Test \\
4 & Flex and Short Beam Shear-Unused \\
5 & DCB, Longitudinal Delam, Cocure-Unused \\
6 & DCB, Longitudinal Delam, Post-Bond \\
7 & DCB, Longitudinal Delam, Cocure \\
8 & DCB, Longitudinal Delam, Post-Bond \\
9 & DCB, Transverse Delam, Cocure \\
10 & DCB, Transverse Delam, Post-Bond \\
11 & DCB, Longitudinal Delam, Cocure \\
12 & Flex and Short Beam Shear, Cocure \\
13 & Flex and Short Beam Shear, Post-Bond \\
\hline
\end{tabular}

\subsection{Tensile Testing}

ASTM D3039 is the standard test method for tensile properties of fiber/matrix composites. Properties obtained from this test are listed in Table 1.2. These properties cannot all be obtained from one specimen because load is only applied in one direction. Longitudinal and transverse pull tests must both be conducted if the fibers running in the two directions are not the same. Since the cloth used in this study consists of identical weft and fill yarns, testing can be done purely in the longitudinal direction. The transverse properties will be identical to those found using a longitudinal test.

\subsubsection{Specimen Preparation}

Typical specimen geometry is shown in Figure 3.3. Each coupon from plate 1 was cut to approximately 0.4 in wide and 9.75in long, consisting of four layers of cloth stacked symmetrically with the longitudinal fibers aligned in the pull direction. The co-cured, four layer laminate resulted in an average plate thickness of 0.0661in. Dimensions for each specimen are listed in Table 3.2. Grip tabs were added to the ends of the specimens to aid in load transfer. The tabs and coupons were lightly sanded and cleaned with acetone at the bond area and $3 \mathrm{M}^{\circledR}$ DP-460 epoxy was used to bond the tabs. At least 24 hours of cure time 
was allowed before testing of the specimens.

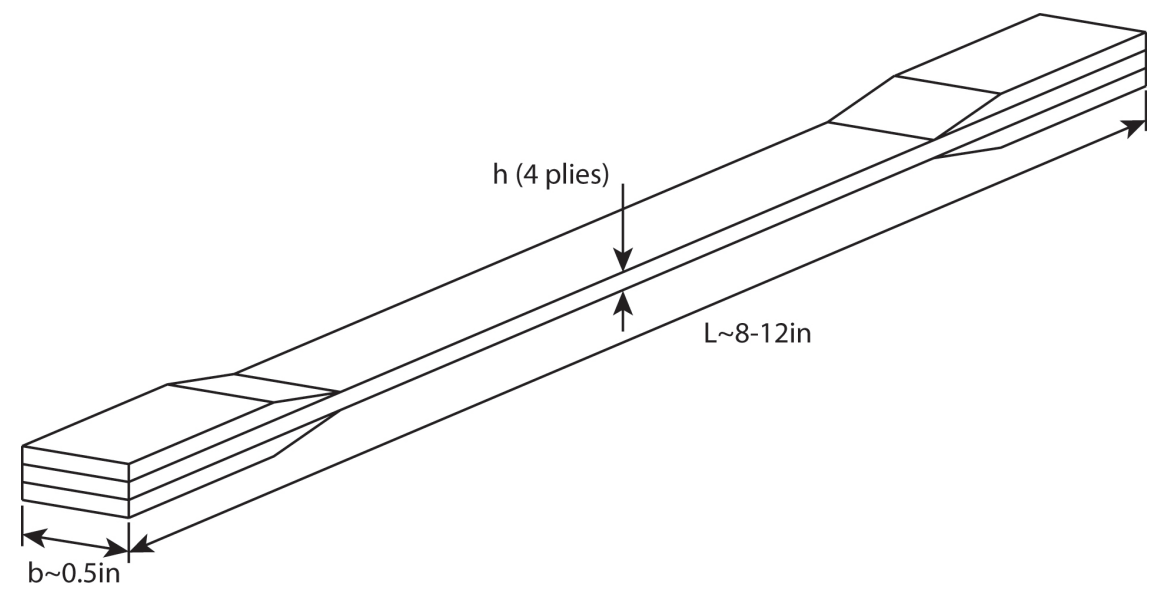

Figure 3.3: Typical tensile coupon geometry

Table 3.2: Tensile specimen average dimensions (inches)

\begin{tabular}{cccc}
\hline \hline Specimen Designation & $L$ & $b$ & $h$ \\
\hline $1-2$ & 9.75 & 0.396 & 0.0661 \\
$1-3$ & 9.75 & 0.341 & 0.0661 \\
$1-5$ & 9.75 & 0.396 & 0.0661 \\
$1-6$ & 9.75 & 0.410 & 0.0654 \\
\hline
\end{tabular}

It is important to note that the symmetry of the laminate is not trivial even though each lamina is aligned with the longitudinal fibers in the pull direction. WFCs with harness satin weaves are not, in themselves, symmetric. The fill yarns dominate the two sides of the laminae, as was shown in Figure 1.7, causing each laminae to act almost like two layers of UDC stacked together and oriented at $90^{\circ}$ to each other. In order to ensure a symmetric laminate with harness satin cloth, the symmetry plane of the laminate must correspond to an alignment of the fill yarns. Figure 3.4 shows an example of a symmetric laminate structure for harness satin cloth. The dark gray side of the sheets correspond to fill yarns in the longitudinal direction. The light gray sides correspond to fill yarns in the transverse direction. 


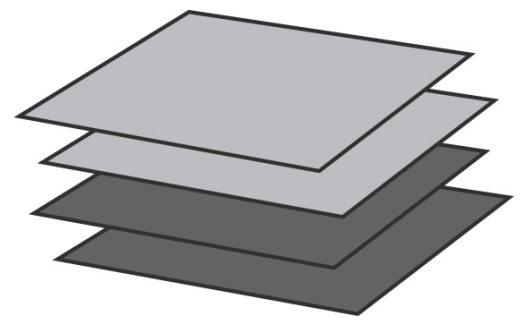

Figure 3.4: Symmetric laminate structure for harness satin weaves

Strain gages were applied to the specimens to measure strain in, and normal to, the pull direction, see Figure 3.5. Application of the gages followed the specifications put forward by Vishay Micro-Measurements ${ }^{\circledR}[64]$.

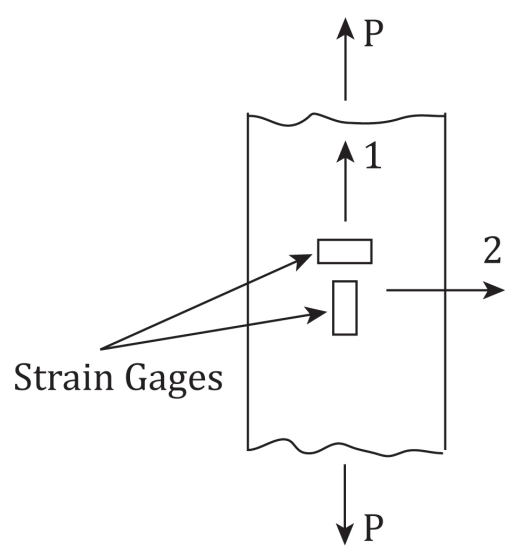

Figure 3.5: Strain gage locations for the tensile test specimens

\subsubsection{Machinery and Instrumentation}

The tensile specimens were mounted vertically in a model 1131 Instron ${ }^{\circledR}$ with hydraulic wedge grips. Load and displacement outputs from the Instron ${ }^{\circledR}$, along with the two strain readings, were input into a LabVIEW ${ }^{\mathrm{TM}}$ interface and recorded. A displacement-controlled waveform of $0.001 \mathrm{in} / \mathrm{sec}$ was used to apply the tensile load. The test ran until ultimate failure was reached. 


\subsubsection{Test Procedure}

The following procedure is an abbreviated version of the steps listed in ASTM D3039 [8].

1. Tighten the lower hydraulic grip on the test specimen, ensuring proper alignment of the fibers in the pull direction

2. Connect the leads from the strain gages to the data acquisition hardware

3. Begin the data acquisition software

4. Clamp the upper hydraulic grip

5. Apply the load through a constant displacement rate of $0.001 \mathrm{in} / \mathrm{sec}$

6. Halt test and data acquisition after failure and remove specimen from test machine

\subsubsection{Calculations}

The Poisson's ratio can be found by plotting the transverse strain on the ordinate and the longitudinal strain on the abscissa. The resulting least-squares fit to this data set will have a slope equal to the Poisson's ratio, as shown in Figure 3.6,

$$
\nu_{12}=\frac{-\Delta \epsilon_{1}}{\Delta \epsilon_{2}}
$$

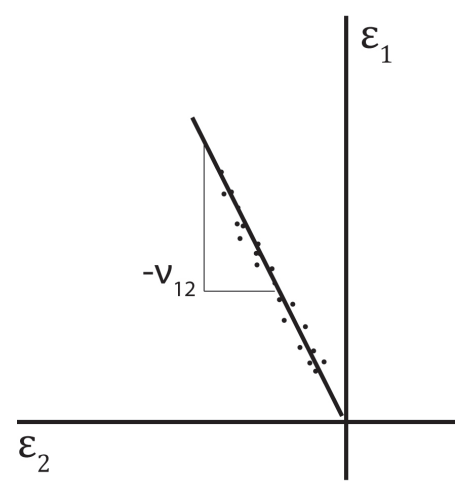

Figure 3.6: Poisson's ratio for longitudinal tensile test

The modulus of elasticity in the longitudinal direction, $E_{1}$, can be found by using Hooke's law which, for linear elastic materials, states that the stress applied to a body may 
be directly related to the resulting strain through the modulus of elasticity:

$$
\sigma_{1}=E_{1} \epsilon_{1},
$$

so $E_{1}$ can be written as

$$
E_{1}=\frac{\Delta \sigma_{1}}{\Delta \epsilon_{1}}
$$

The stress applied to the tensile specimen of uniform cross section is equal to the applied load divided by the cross sectional area over which it acts:

$$
\sigma_{1}=\frac{P}{h b} .
$$

The longitudinal tensile strength is defined as the maximum stress before failure:

$$
F_{1 t}=\frac{P_{c}}{h b},
$$

where $P_{c}$ is the critical applied load which causes laminate failure. Ultimate longitudinal tensile failure strain cannot be determined from the strain readings because the adhesive joining the strain gages to the composite specimen fails at a lower strain than the composite. An extrapolation must be made on a plot of normal stress vs. normal strain, as can be seen in Figure 3.7.

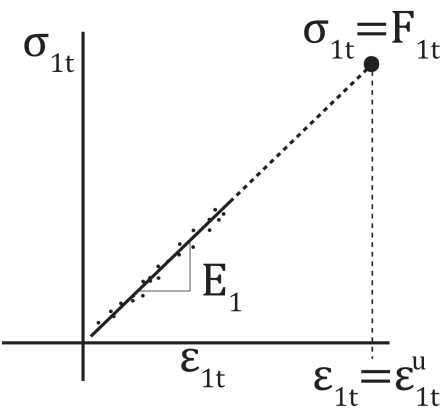

Figure 3.7: Extrapolation for ultimate longitudinal tensile failure strain 
Since the weft and fill yarns are identical, as stated previously, the properties calculated for the longitudinal yarns can be used for the transverse yarns as well, so

$$
\begin{aligned}
& E_{2}=E_{1}, \\
& F_{2 t}=F_{1 t}, \\
& \epsilon_{2 t}^{u}=\epsilon_{1 t}^{u},
\end{aligned}
$$

and, through the relationship of the major and minor Poisson's ratios:

$$
\begin{gathered}
\nu_{21}=\frac{E_{2}}{E_{1}} \nu_{12}, \\
\nu_{21}=\nu_{12} .
\end{gathered}
$$

\subsection{In-Plane Shear Testing}

There are three possible shear moduli which can be obtained from shear testing: the inplane shear modulus, $G_{12}$, and the two out of plane moduli, $G_{13}$ and $G_{23}$. Each of these moduli have corresponding shear strengths, $F_{6}, F_{5}$, and $F_{4}$ with ultimate shear strains $\gamma_{12}^{u}$, $\gamma_{13}^{u}$, and $\gamma_{23}^{u}$, respectively. An in-plane shear test, by definition, is a test in which shear

distortion occurs only in the plane of the composite sheet [13]. ASTM D3518 is one of the standard test methods for in-plane shear testing of fiber/matrix composites and will yield accurate results for $G_{12}$ but not for $\gamma_{12}^{u}$ or $F_{6}$ because the laminate is not entirely in pure shear.

\subsubsection{Specimen Preparation}

Typical specimen geometry is shown in Figure 3.8. Each coupon from plate 3 was cut to approximately $1.0 \mathrm{in}$ wide, and $9.75 \mathrm{in}$ long, with the layup schedule $[+45,-45]_{2 s}$. The cocured, eight layer laminate resulted in an average plate thickness of 0.1364in. Dimensions for each specimen are listed in Table 3.3. 


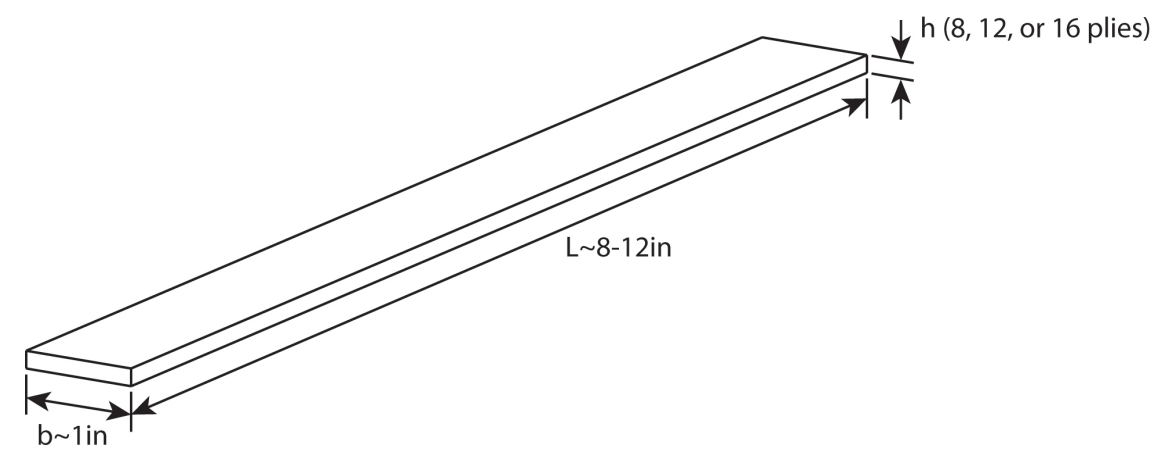

Figure 3.8: Typical $\pm 45^{\circ}$ shear coupon geometry

Table 3.3: In-plane shear specimen average dimensions (inches)

\begin{tabular}{cccc}
\hline \hline Specimen Designation & $L$ & $b$ & $h$ \\
\hline $3-1$ & 9.75 & 1.015 & 0.1366 \\
$3-2$ & 9.75 & 0.965 & 0.1385 \\
$3-3$ & 9.75 & 0.987 & 0.1386 \\
$3-4$ & 9.75 & 1.015 & 0.1357 \\
$3-5$ & 9.75 & 0.974 & 0.1347 \\
$3-6$ & 9.75 & 1.002 & 0.1393 \\
$3-7$ & 9.75 & 1.010 & 0.1312 \\
\hline
\end{tabular}

Strain gages were applied to the specimens to measure strain in, and normal to, the pull direction, see Figure 3.9 [5]. Application of the gages followed the instructions supplied by Vishay Micro-Measurements ${ }^{\circledR}[64]$.

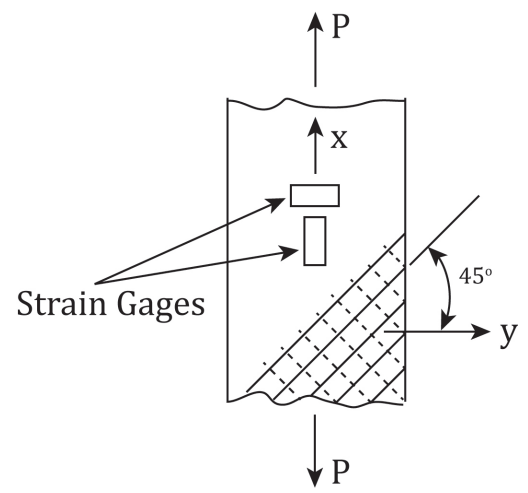

Figure 3.9: Strain gage locations for the in-plane shear test specimens 


\subsubsection{Machinery and Instrumentation}

The in-plane shear specimens were mounted vertically in a model 1131 Instron ${ }^{\circledR}$ with hydraulic wedge grips. Load and displacement outputs from the Instron ${ }^{\circledR}$, along with the two strain readings, were input into a LabVIEW ${ }^{\mathrm{TM}}$ interface and recorded. A displacementcontrolled waveform of $0.001 \mathrm{in} / \mathrm{sec}$ was used to apply the tensile load. The test ran until ultimate failure was reached.

\subsubsection{Test Procedure}

The procedure for the in-plane shear test is identical to that of the tensile test in $\S 3.3 .3$.

\subsubsection{Calculations}

The goal of the in-plane shear test is to calculate $G_{12}$, which is the ratio of shear stress, $\tau_{12}$, to shear strain, $\gamma_{12} \cdot \tau_{12}$ can be found through a coordinate transformation from $x-y$ coordinates to the composite material coordinates:

$$
\{\sigma\}_{12}=[T]_{1}\{\sigma\}_{x y},
$$

where $[T]_{1}$ is the stress transformation tensor:

$$
[T]_{1}=\left[\begin{array}{ccc}
\cos ^{2}(\theta) & \sin ^{2}(\theta) & 2 \sin (\theta) \cos (\theta) \\
\sin ^{2}(\theta) & \cos ^{2}(\theta) & -2 \sin (\theta) \cos (\theta) \\
-\sin (\theta) \cos (\theta) & \sin (\theta) \cos (\theta) & \cos ^{2}(\theta)-\sin ^{2}(\theta)
\end{array}\right]
$$

Knowing that $\theta=-45^{\circ}$, and $\sigma_{y}=\tau_{x y}=0$ :

$$
\left\{\begin{array}{c}
\sigma_{1} \\
\sigma_{2} \\
\tau_{12}
\end{array}\right\}=\left[\begin{array}{ccc}
0.5 & 0.5 & -1.0 \\
0.5 & 0.5 & 1.0 \\
0.5 & -0.5 & 0.0
\end{array}\right]\left\{\begin{array}{c}
\frac{P}{h b} \\
0 \\
0
\end{array}\right\} .
$$


This can be simplified to solve directly for $\tau_{12}$ :

$$
\tau_{12}=\frac{P}{2 h b}
$$

The shear strain, $\gamma_{12}$ can be found in a similar manner:

$$
\{\epsilon\}_{12}=[T]_{2}\{\epsilon\}_{x y},
$$

where $[T]_{2}$ is the strain transformation tensor:

$$
[T]_{2}=\left[\begin{array}{ccc}
\cos ^{2}(\theta) & \sin ^{2}(\theta) & \sin (\theta) \cos (\theta) \\
\sin ^{2}(\theta) & \cos ^{2}(\theta) & -\sin (\theta) \cos (\theta) \\
-2 \sin (\theta) \cos (\theta) & 2 \sin (\theta) \cos (\theta) & \cos ^{2}(\theta)-\sin ^{2}(\theta)
\end{array}\right]
$$

Values of $\epsilon_{x}$ and $\epsilon_{y}$ are known from the strain gages, so

$$
\left\{\begin{array}{c}
\epsilon_{1} \\
\epsilon_{2} \\
\gamma_{12}
\end{array}\right\}=\left[\begin{array}{ccc}
0.5 & 0.5 & -0.5 \\
0.5 & 0.5 & 0.5 \\
1.0 & -1.0 & 0.0
\end{array}\right]\left\{\begin{array}{c}
\epsilon_{x} \\
\epsilon_{y} \\
\gamma_{x y}
\end{array}\right\}
$$

This can be simplified to solve directly for $\gamma_{12}$ with known values of $\epsilon_{x}$ and $\epsilon_{y}$ :

$$
\gamma_{12}=\epsilon_{x}-\epsilon_{y}
$$

$G_{12}$ can now be found by finding the slope of a least squares fit to the plot of $\tau_{12}$ on the abscissa and $\gamma_{12}$ on the ordinate, as shown in Figure 3.10. ASTM D3518 recommends using a shear strain range of $4000 \pm 200 \mu \epsilon$ starting between 1500 and $2500 \mu \epsilon$ [5].

\subsection{Short Beam Shear Testing}

The short beam shear test is based on simple mechanics of materials equations which do not account for the complex stress state that exists at the point of load application, as 


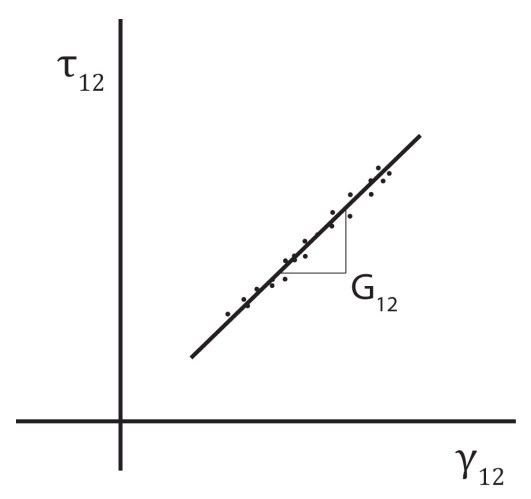

Figure 3.10: The Shear Modulus, $G_{12}$, from the in-plane shear test

shown by Whitney $[1,68]$. This method should only be used as a screening tool to make comparisons between laminate structures or fiber/matrix materials [32]. It will be used in this study to compare the relative interlaminar strengths of co-cured and post-bonded composite laminates.

ASTM D2344 is the standard test method for short beam shear (SBS) testing of highmodulus fiber/matrix composites. The SBS test involves the use of a short composite beam loaded in a three-point bend fixture. Normal and shear stress will arise from the loading condition and, as long as a correct span to depth ratio for the beam is used, shear failure will be induced before failure due to normal stress [32]. The shear and bending moment diagram for a beam under three-point bending is shown in Figure 3.11. This fixture is useful for interlaminar shear failure because the vertical shear force remains constant throughout the beam except at the point where the load is applied. Designing the beam to meet the requirement of shear-induced failure rather than normal stress-induced failure is also fairly simple as long as the dimension requirements in Figure 3.12 are considered and a proper span to thickness ratio is used. ASTM D2344 suggests a span to thickness ratio of 4 [3]. 


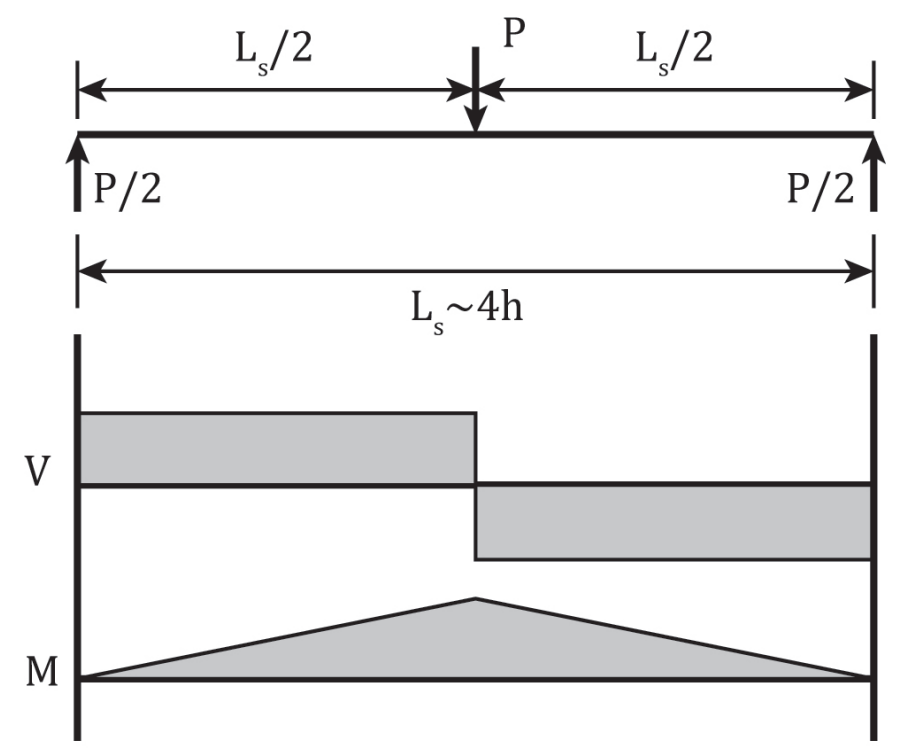

Figure 3.11: Shear and bending moment diagram for three-point bend test

\subsubsection{Specimen Preparation}

Typical specimen geometry is shown in Figure 3.12. Each specimen was cut to approximately 0.33in wide and 1.0in long from plates 12 and 13. Recall from Table 3.1 that plate 12 is a co-cured plate, and plate 13 is a post-bonded plate. Both plates have the layup schedule $[90]_{5 s}$, yielding a symmetric midplane with fill yarns aligned with the longitudinal axis of the beam. Dimensions for each specimen are listed in Table 3.4.

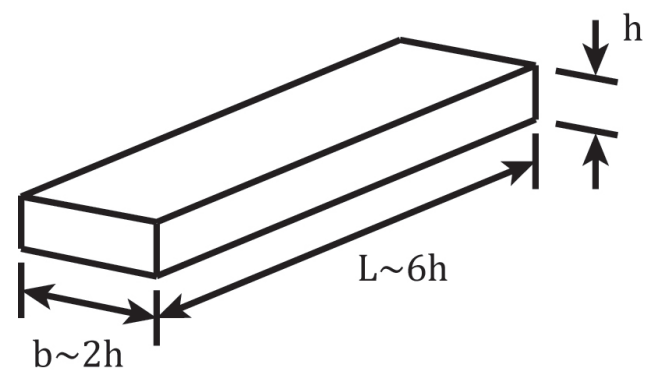

Figure 3.12: Typical short beam shear specimen geometry 
Table 3.4: Short beam shear specimen average dimensions (inches)

\begin{tabular}{cccc}
\hline \hline Specimen Designation & $L$ & $b$ & $h$ \\
\hline $12-5$ & 1.014 & 0.335 & 0.1695 \\
$12-6$ & 1.018 & 0.335 & 0.1690 \\
$12-7$ & 1.018 & 0.334 & 0.1690 \\
$12-8$ & 1.018 & 0.334 & 0.1682 \\
$12-9$ & 1.012 & 0.326 & 0.1694 \\
$12-10$ & 1.017 & 0.326 & 0.1681 \\
$13-5$ & 1.018 & 0.330 & 0.1705 \\
$13-6$ & 1.011 & 0.331 & 0.1700 \\
$13-7$ & 1.010 & 0.333 & 0.1716 \\
$13-8$ & 1.005 & 0.333 & 0.1720 \\
$13-9$ & 1.038 & 0.332 & 0.1686 \\
$13-10$ & 1.011 & 0.334 & 0.1708 \\
\hline
\end{tabular}

\subsubsection{Machinery and Instrumentation}

The specimens were mounted in a three-point bend fixture and loaded using a model 1131 Instron ${ }^{\circledR}$ with a displacement-controlled compressive waveform of $0.001 \mathrm{in} / \mathrm{sec}$. Load and displacement outputs from the Instron ${ }^{\circledR}$ were input into a LabVIEW ${ }^{\mathrm{TM}}$ interface and recorded. Each test ran until ultimate failure was reached.

\subsubsection{Test Procedure}

The following procedure is an abbreviated version of the steps listed in ASTM D2344 [3].

1. Place specimen in the three-point bend fixture on the test machine and align it so that the longitudinal axis is perpendicular to the loading nose and side supports

2. Begin the data acquisition software

3. Apply the load through a constant displacement rate of $0.001 \mathrm{in} / \mathrm{sec}$

4. Halt the test and data acquisition after failure and remove the specimen from the three-point bend fixture.

\subsubsection{Calculations}

The desired value from the SBS test is $F^{s b s}$, the short beam shear strength, which is the maximum value of shear stress, $\tau_{12}$, reached before failure. The calculation of shear stress for a beam in bending, assuming a homogeneous beam of uniform cross section, follows a 
mechanics of materials formulation. The following steps are summarized from [57].

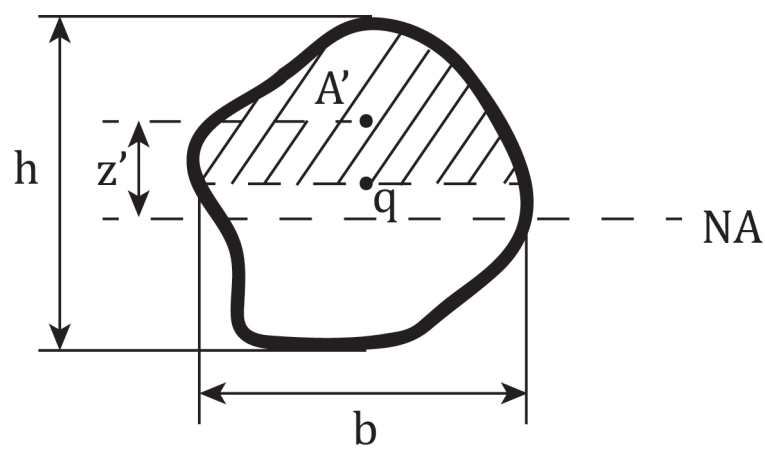

Figure 3.13: Beam section through q

$$
\tau_{12}=\frac{V A^{\prime} z^{\prime}}{I b}
$$

where, at $q$, a point within the cross section where values are desired, $V$ is the vertical shear, $A^{\prime}$ is the area of the beam cross section above $q, z^{\prime}$ is the distance from the neutral axis to the centroid of $A^{\prime}$, and $b$ is the width of the cross section. Maximum shear stress will occur at the neutral axis; therefore, for the beam in Figure 3.12, $z^{\prime}=h / 4, A^{\prime}=b h / 2$, and $I=b h^{3} / 12$. Substituting these into Equation 3.19 yields

$$
\tau_{12}=\frac{3}{2} \frac{P}{b h}
$$

The short beam shear strength will correspond to the maximum load seen by the specimen:

$$
F^{s b s}=\frac{3}{2} \frac{P_{\max }}{b h} .
$$




\subsection{Flexural Testing}

Due to the anisotropy of composite laminate beams, as opposed to metal beams, and the complex transfer of stress and strain between laminae during bending, a composite beam may have a different modulus of elasticity for bending applications than it would for axial loading. Normally, when analyzing a beam in bending, the modulus of elasticity of the material is used in equations like 2.14 to find the deflection of a cantilever beam. In composite analysis, the modulus corresponding to the longitudinal axis of the beam is used, which may be adequate for unidirectional laminates with all plies oriented either in, or normal to, the longitudinal axis of the beam. Many other composite beams, though, require a more complicated formulation of the modulus, commonly known as the flexural modulus, $E_{f}$. Several theoretical models can be used to find appropriate values for $E_{f}$ based on composite laminate theory [32]. $E_{f}$ can also be found through testing of laminates. ASTM D7264 is the standard test method for flexural properties of fiber/matrix composites. Another standard for flexural properties, D790, is also commonly used for composite materials.

There are two choices of fixtures for flexural testing: the three-point bend fixture used in SBS testing, or a four-point bend fixture. The difference in the shear and bending moment diagrams for these two fixtures can be seen in Figure 3.14. The failure desired for the determination of $E_{f}$ should be induced by a pure normal stress, meaning the only force acting on the beam should be a moment. If the three point bending test is used, the maximum moment occurs at a point of asymptotic shear force, making the stress state a combination of normal and shear stress. The four-point bending test will produce a constant moment, with zero shear, in between the load application points, leading to a state of constant pure normal stress. The four-point bending test, for this reason, was chosen for flexural testing of the composite laminates in this study. The span to thickness ratio recommended by ASTM D7264 is 32:1, but optional ratio include 16:1, 20:1, 40:1, and 60:1 [7]. A span of 16:1 was used for all specimens. 

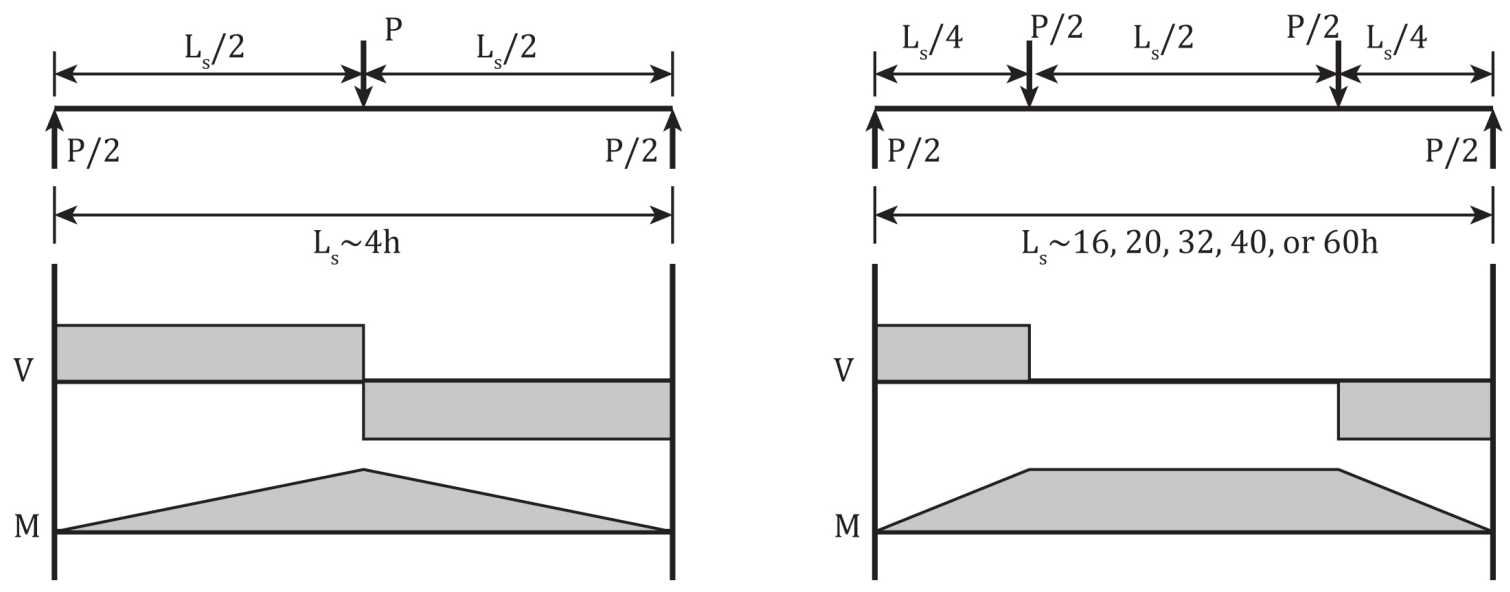

Figure 3.14: Comparison of three and four point shear and bending moment diagrams

\subsubsection{Specimen Preparation}

Typical specimen geometry is shown in Figure 3.15. Each coupon was cut to approximately 0.5 in wide with a length at least $20 \%$ longer than the span from plates 12 and 13 with average thicknesses of 0.17in. The support span for all tests was 2.64in and the load span was 1.32in.

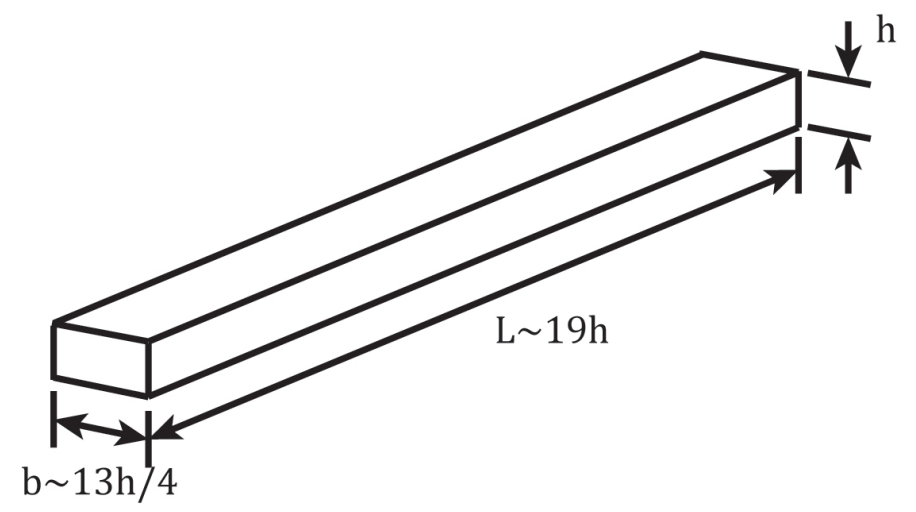

Figure 3.15: Typical geometry for flex specimens

Recall from Table 3.1 and $\S 3.5$ that plate 12 is a co-cured laminate, and 13 is postbonded. both plates have the layup schedule $[90]_{5 s}$, yielding a symmetric midplane with weft yarns running along the longitudinal axis of the beam. Dimensions for the flex speci- 
mens are listed in Table 3.5.

Table 3.5: Flex specimen average dimensions (inches)

\begin{tabular}{cccc}
\hline \hline Specimen Designation & $L$ & $b$ & $h$ \\
\hline $12-1$ & 3.280 & 0.529 & 0.1687 \\
$12-2$ & 3.280 & 0.549 & 0.1668 \\
$12-3$ & 3.280 & 0.559 & 0.1696 \\
$12-4$ & 3.278 & 0.541 & 0.1700 \\
$12-11$ & 3.273 & 0.532 & 0.1679 \\
$12-12$ & 3.277 & 0.538 & 0.1691 \\
$13-1$ & 3.264 & 0.548 & 0.1704 \\
$13-2$ & 3.258 & 0.555 & 0.1702 \\
$13-3$ & 3.269 & 0.546 & 0.1719 \\
$13-4$ & 3.272 & 0.579 & 0.1725 \\
$13-11$ & 3.251 & 0.539 & 0.1713 \\
$13-12$ & 3.255 & 0.530 & 0.1709 \\
\hline
\end{tabular}

\subsubsection{Machinery and Instrumentation}

The Cal Poly composites lab was not equipped with a four point bend fixture. A new fixture had to be designed for the testing of the flex specimens. The spacing of the vertical rails was designed to match the spacing of the Wyoming Test Fixtures ${ }^{\circledR}$ three-point bend apparatus. A schematic of the four-point bend fixture can be found in Appendix A.

The displacement for the flex testing was output from a linear variable differential transformer (LVDT). The exterior LVDT was needed for the testing of the DCB specimens to minimize any stair-stepping in the $P-\delta$ plot which might result from the high-range LVDT built into the Instron ${ }^{\circledR}$. The exterior used was a Schlumberger ${ }^{\circledR} 847624$ with 1.5 in of travel. The most linear range was between $0.4 \mathrm{in}$ and $1.5 \mathrm{in}$ of travel. The calibration curve for the LVDT can be seen in Figure 3.16.

The specimens were mounted in a four-point bend fixture and loaded using a model 1131 Instron ${ }^{\circledR}$ with a displacement-controlled compressive waveform of $0.001 \mathrm{in} / \mathrm{sec}$. Load was output from the Instron ${ }^{\circledR}$ and displacement was output from the LVDT. These were 


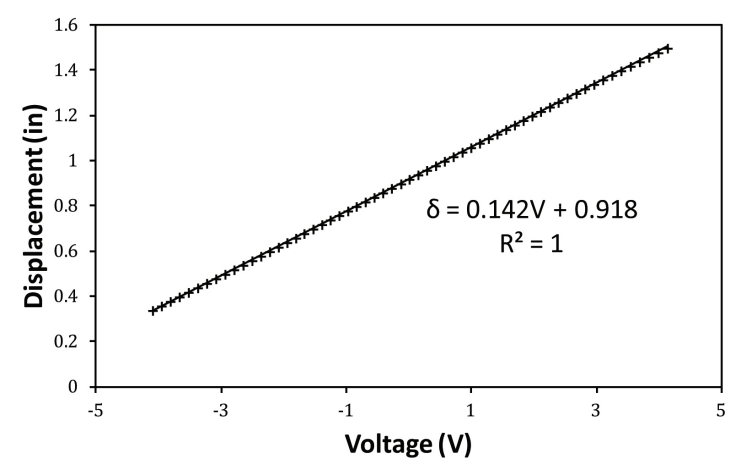

Figure 3.16: LVDT calibration chart

input into a LabVIEW ${ }^{\mathrm{TM}}$ interface and recorded. Each test ran until ultimate failure was reached.

\subsubsection{Test Procedure}

The procedure for the flex testing is identical to the procedure for the SBS testing in $\S$ 3.5.3 with the substitution of the four-point bend fixture in place of the three-point.

\subsubsection{Calculations}

The maximum moment for the four-point bend test can be calculated by finding the area under the left half of the shear diagram in Figure 3.14

$$
M_{\max }=\frac{P L_{s}}{8} .
$$

The stress in a beam due to a moment is

$$
\sigma=\frac{M \frac{h}{2}}{I}
$$

yielding

$$
\sigma=\frac{3 P L_{s}}{4 b h^{2}}
$$


where $L_{s}$ is the span width.

The maximum strain in the beam can be expressed as

$$
\epsilon=\frac{4.36 \delta h}{L_{s}^{2}}
$$

From Hooke's law,

$$
\sigma=E_{f} \epsilon,
$$

so $E_{f}$ can be found by plotting $\sigma$ on the abscissa with $\epsilon$ on the ordinate and finding the slope of the least-squares fit to the data:

$$
E_{f}=\frac{\Delta \sigma}{\Delta \epsilon}
$$

\subsection{DCB Testing}

The importance of the DCB test and, likewise, mode I fracture toughness determination was discussed in Chapter 1. This section will focus on the preparation required for DCB testing, including specimen/fixture design and manufacturing, and machinery adjustments and additions.

\subsubsection{Specimen Preparation}

Typical specimen geometry is shown in Figure 3.17. DCB coupons were cut from plates 5 through 11 . Recall from Table 3.1 that plates $5,7,9$, and 11 were co-cured plates with the layup schedule $[0]_{4 s}$ and plates 6,8 , and 10 were post-bonded plates with the layup schedule $[90]_{4 s}$. Plates $5,6,7,8$ and 11 had delaminations built to propagate along fill yarns and plates 9 and 10 had delaminations built to propagate along weft yarns. Plate 5 was created for practice purposes and six coupons were cut from it to test the usefulness of the Instron's ${ }^{\circledR}$ built in load cell. Dimensions for each specimen can be found in Appendix B.5.

The layup process for the co-cured plates consisted of aligning the first four layers in the

correct orientation, placing a 0.0005 in thick Polytetrafluoroethylene(Teflon $\left.{ }^{\circledR}\right)$ insert on the 


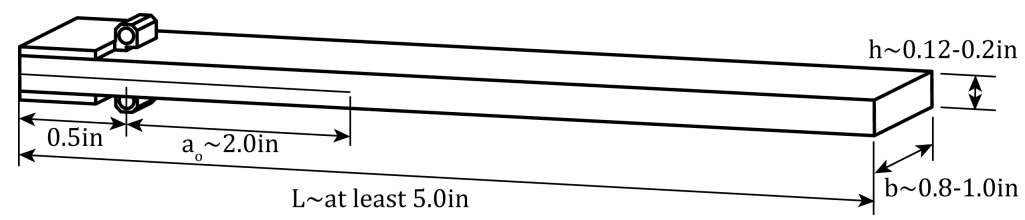

Figure 3.17: Typical geometry for DCB specimens

carbon where the delamination was desired. The next four layers were placed on top of the insert in the correct orientation, ensuring mirror symmetry about the laminate midplane and each cantilevered beam's midplane. After the layup was complete, the laminate was put into the autoclave with the cure cycle outlined in Figure 3.1. The layup process for the post-bond plates consisted of aligning the first four layers of cloth, ensuring mirror symmetry, and fully curing them in the autoclave. The delamination was inserted in the laminate by following the process specified in Chen et. al's patent [25]. Once the delamination was placed, the final four layers were carefully laid over the first four and put into the autoclave for curing.

Specimens and loading hinges were sanded and cleaned with acetone to increase bond strength. Three resin systems were tested for this steel-carbon bond: West System 205 fast cure epoxy, System Three 1000K10 Quick Cure Epoxy, and 3M ${ }^{\mathrm{TM}}$ Scotch-Weld ${ }^{\mathrm{TM}}$ DP460 structural adhesive. The West System and System Three bonds were air-dried, per manufacturer's instructions, and the $3 \mathrm{M}^{\mathrm{TM}}$ bond was cured in an oven at $160^{\circ} \mathrm{F}$ for $15 \mathrm{~min}$. Testing of each bond on the specimens from plate 5 indicated that the West System and System Three epoxies were not strong enough to resist the required load. The 3M bond did not show any signs of failure.

\subsubsection{Machinery and Instrumentation}

The DCB test requires a load application that remains perpendicular to the direction of delamination. In order to achieve this, piano hinges are typically attached to the ends of the DCB and screwed into a fixture mounted in the Instron ${ }^{\circledR}$. According to ASTM D5528, the hinges should be at least as wide as the DCB specimen, made of metal, and capable of 
resisting the applied load [6]. The maximum load can be calculated with:

$$
P_{\max }=\frac{b}{a} \sqrt{\frac{h^{3} E_{1} G_{I c}}{96}}
$$

Average values of $G_{I c}$ for several materials can be found in [32]. Assumptions made for the calculation of maximum load were: $E_{1}=1.4 \times 10^{11} \mathrm{~N} / \mathrm{m}^{2}, G_{I c}=222 \mathrm{~J} / \mathrm{m}^{2}, a=25 \mathrm{~mm}$, $b=25 \mathrm{~mm}$, and $h=5 \mathrm{~mm}$. Inserting these values into Equation 3.27 yields a max load of about $400 \mathrm{~N}$ with a factor of safety of 2 . The goal of the hinge design was to create an easy to manufacture, reusable hinge that would deflect less than 0.001in. The hinge should also have a removable pin to decrease any compression of the specimen which might occur during clamping of the hydraulic grips. After iteration of pin choices, pivot-pin clearance, and overall dimensions of the pivot area, the preliminary hinge design was finalized. Figure 1.3 shows the assembly of the hinges which were used in testing plate 5 to verify testing procedure and design. The bondable hinges can be easily reused by locally heating the steel with an oxy-acetylene torch for about two seconds. This amount of heat allows the epoxy holding the hinge in place to degrade without any adverse material effects on the hinge. It is unclear how much damage is done to the DCB specimen in this heating process. SEM analysis is not recommended after this step. Schematics for the bondable hinge and the upper and lower jaw hinges can be found in Appendix C.

Testing of plate 5 revealed erratic load data due to the Instron's ${ }^{\circledR}$ high load capacity. The Instron's ${ }^{\circledR}$ built in load-cell can measure loads from 0 to $22,000 \mathrm{lbf}$, making it very useful for test series involving load swings of several thousand pounds. DCB testing, however, requires high accuracy load measurements with a range of 0 to 20lbs. A test of the load output from the Instron ${ }^{\circledR}$ was performed by adding masses of 1000,1500, 2000, 2500, and 3000grams and measuring the corresponding load output. The average difference between the added weight and the output load was $1.3 \mathrm{lbf}$, a $6 \%$ error for the expected range in DCB testing. An Omega ${ }^{\circledR}$ LCH-100 load cell with a range of 0-100lbf was chosen for use instead of the built in load cell. The LCH-100 was mounted by bolting it to a fixture clamped in the upper Instron ${ }^{\circledR}$ jaw. Schematics for the upper-jaw hinge, the bondable hinges, and the 
lower-jaw hinge can be found in Appendix C. The load cell must be calibrated before each test to ensure correct load readings. The calibration procedure can be found in Appendix E.

Crack propagation was measured through visual observation of the crack tip through a microscope. In order to view the crack tip easier, the sides of each specimen were coated with silver Sharpie ${ }^{\mathrm{TM}}$. Markings were made at $1 \mathrm{~mm}$ increments along the side of the specimen using a laser engraving machine, as seen in Figure 3.18. The microscope was mounted to the Instron ${ }^{\circledR}$ using a custom-machined mounting bracket which allows for easy installation and 3-axis movement of the microscope lens. Images of the mount can be found in Appendix D.

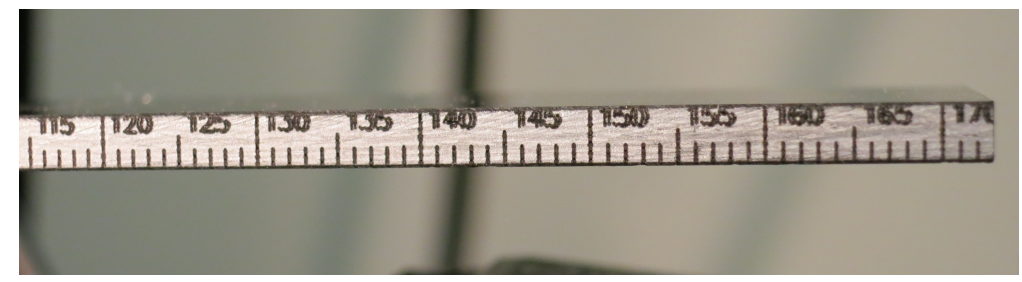

Figure 3.18: Laser-engraved crack propagation markings

\subsubsection{Test Procedure}

The following test procedure is a slightly modified summarization of the procedure listed in ASTM D5528 [6].

1. Mount the hinges in the Instron ${ }^{\circledR}$ grips and connect the lower-jaw hinge to the bottom bondable hinge using one of the steel pins

2. Set the microscope in a position to observe the location of the delamination front with an accuracy of $\pm 0.5 \mathrm{~mm}$

3. Start the data acquisition software, ensuring proper connections of the LVDT and the exterior load-cell

4. Connect the upper-jaw hinge to the upper bondable hinge using one of the steel pins

5. Initial Loading:

a Load at a constant displacement of $0.001 \mathrm{in} / \mathrm{sec}$. Load and displacement gains do not have to be set at the Instron ${ }^{\circledR}$ controller because the external load cell and LVDT will measure accurate loads and displacements 
b Pause loading after $3-5 \mathrm{~mm}$ of crack growth. If unstable delamination growth from the insert was observed, note it in the report. Continue loading for another $3-5 \mathrm{~mm}$

c Pause the load cycle and mark the position of the tip of the precrack on both edges of the specimen. Note in the report if the position on either side differs by more than $2 \mathrm{~mm}$ (this may be an indication of asymmetrical loading)

d Unload the specimen by pressing the single arrow button $\Uparrow$ on the Instron ${ }^{\circledR}$ controller. Unload until the head displacement is close to the initial unloaded position. Do not press the button more than once as this will cause erratic data to be reported

6. Reloading:

a Reload the specimen at the same crosshead rate and record the load and displacement values for delamination onset from the precrack

b Continue crack extension and record as many values as possible. Extend the crack at least $50 \mathrm{~mm}$ past the precrack. If the LVDT reading drops below $0.45 \mathrm{in}$, pause both the data acquisition and the Instron ${ }^{\circledR}$ controller and place a 1in gage block underneath the LVDT plunger to allow for greater travel (this may need to be repeated for large-deflections)

c Unload the specimen by pressing the single arrow button $\Uparrow$ on the Instron ${ }^{\circledR}$ controller. Be sure to remove any added gage blocks before unloading to prevent any damage of the LVDT. If the crack has extended all the way through, this and the following steps may be omitted

d Record the positions of the tip of the delamination on both sides of the specimen. Note any deviation greater than $2 \mathrm{~mm}$

\subsubsection{Calculations}

Calculations for the DCB testing were covered in Chapter 2.

\subsection{Summary of Testing Inputs and Outputs}

This section provides a table of values used to setup the Instron ${ }^{\circledR}$ outputs and waveforms for all of the test series performed in this study. These values are specific to the Cal Poly, SLO composites laboratory and may not be applicable in other testing facilities.

The LabVIEW ${ }^{\mathrm{TM}}$ files, below, can be found in the following directory on the computer in the Cal Poly composites laboratory: C: $\backslash$ Documents and Settings $\backslash$ Administrator $\backslash$ My Documents \DrMello. 
Table 3.6: Inputs and outputs for each test series

\begin{tabular}{cccccc}
\hline \hline Test & LabVIEW vi & $P$ Gain (lbf/V) & $\delta$ Gain (in/V) & $\delta_{\max }($ in $)$ & $\dot{\delta}($ in $/ \mathrm{s})$ \\
\hline Tensile & tensile testing.vi & 800 & LVDT & 2 & 0.001 \\
In-Plane Shear & tensile testing.vi & 800 & NA & 2 & 0.001 \\
SBS & flexSBS.vi & 150 & 0.1 & 1 & -0.001 \\
Flex & flexSBS.vi & 150 & LVDT & 1 & -0.001 \\
DCB & DCB.vi & LCH-100 & LVDT & 4 & 0.001 \\
\hline
\end{tabular}




\section{Chapter4}

\section{Testing Results and Discussion}

\subsection{Tensile and In-Plane Shear Testing}

Tensile and in-plane shear specimens were tested according to ASTM D3039 and D3518, respectively. Four specimens from plate 1 were tested and seven from plate 3 . The purpose of these tests was to determine the modulus of elasticity in the fiber directions, the major and minor Poisson's ratios, the ultimate tensile failure strengths and strains in the fiber directions, and the in-plane shear modulus. Strain and load data from each test were input into a MATLAB ${ }^{\circledR} \mathrm{m}$-file for data analysis. The resulting stress-strain and strain-strain plots can be found in Figures 4.1, 4.2, and 4.3. A summary of the results can be found in Tables 4.1 and 4.2, and the statistical analysis of these results can be found in Table 4.3.

Table 4.2: In-plane shear results

Table 4.1: Tensile results

\begin{tabular}{ccccc}
\hline \hline Specimen & $E_{1}(\mathrm{Msi})$ & $\nu_{12}$ & $F_{1 t}(\mathrm{Ksi})$ & $\epsilon_{1 t}^{u}(\%)$ \\
\hline $1-2$ & 8.155 & 0.054 & 90.060 & 1.104 \\
$1-3$ & 7.849 & 0.064 & 91.389 & 1.164 \\
$1-5$ & 8.134 & 0.045 & 99.228 & 1.220 \\
$1-6$ & 8.014 & 0.048 & 95.443 & 1.191
\end{tabular}

\begin{tabular}{cc}
\hline \hline Specimen & $G_{12}(\mathrm{Msi})$ \\
\hline $3-1$ & 0.484 \\
$3-2$ & 0.471 \\
$3-3$ & 0.484 \\
$3-4$ & 0.440 \\
$3-5$ & 0.473 \\
$3-6$ & 0.479 \\
$3-7$ & 0.502
\end{tabular}

The values listed in Table 4.3 are necessary for even the simplest of composite analyses. The fiber-direction moduli of elasticity are much lower for this composite than for many unidirectional carbon/epoxy fabrics. AS4/3501-6, for instance, typically has a modulus of elasticity of $20 \mathrm{Msi}$ in the fiber direction with a failure strength of $310 \mathrm{Ksi}$ at about $1.4 \%$ 


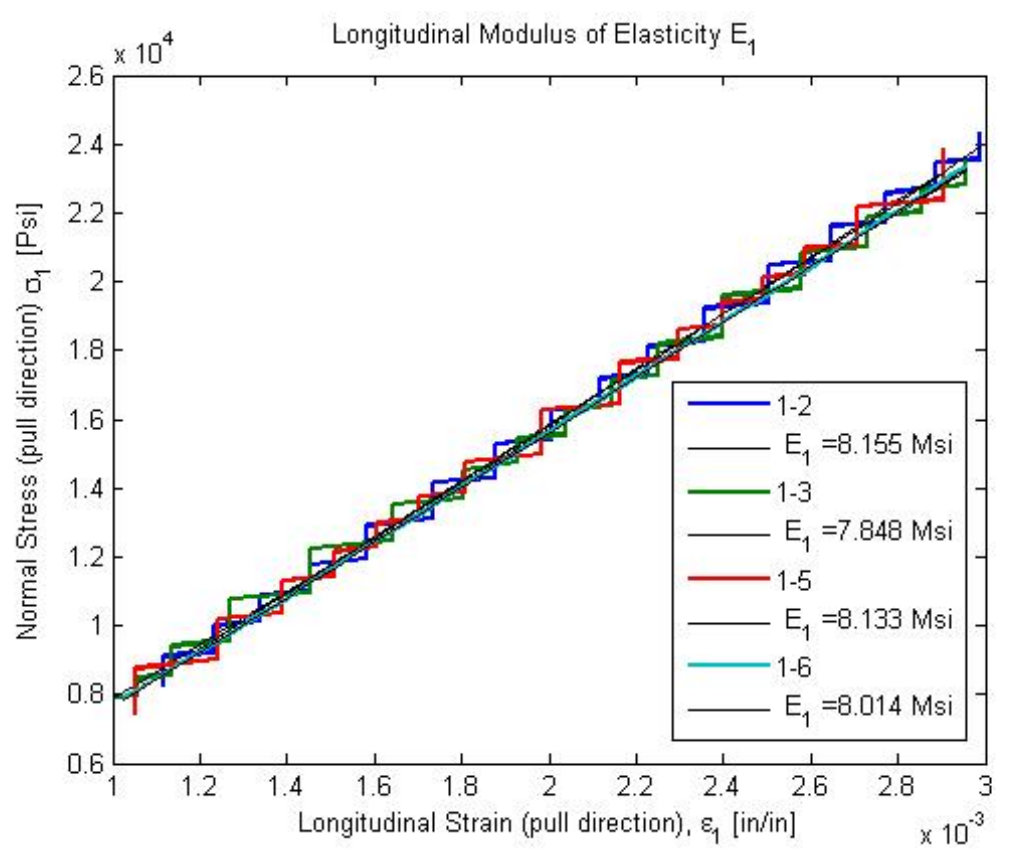

Figure 4.1: Longitudinal modulus of elasticity in the fiber direction

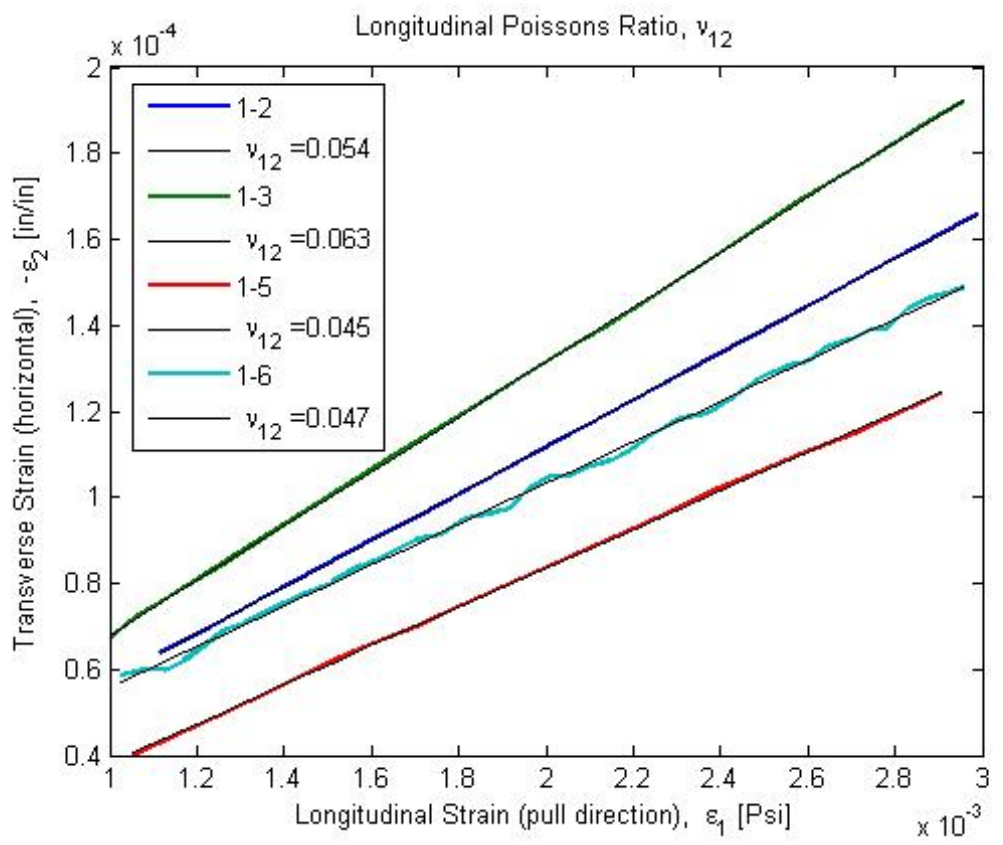

Figure 4.2: Major Poisson's ratio, $\nu_{12}$, from longitudinal pull

strain. The fabric used in this study has a failure strength of $94 \mathrm{Ksi}$ at $1.2 \%$ strain with $E=8.04 \mathrm{Msi}$. These differences can be attributed to the relative number of fibers running in the principal material directions. There will be about half as many fibers running in the longitudinal (and transverse) direction in a 3k WFC than in the fiber direction of a $3 \mathrm{k}$ 


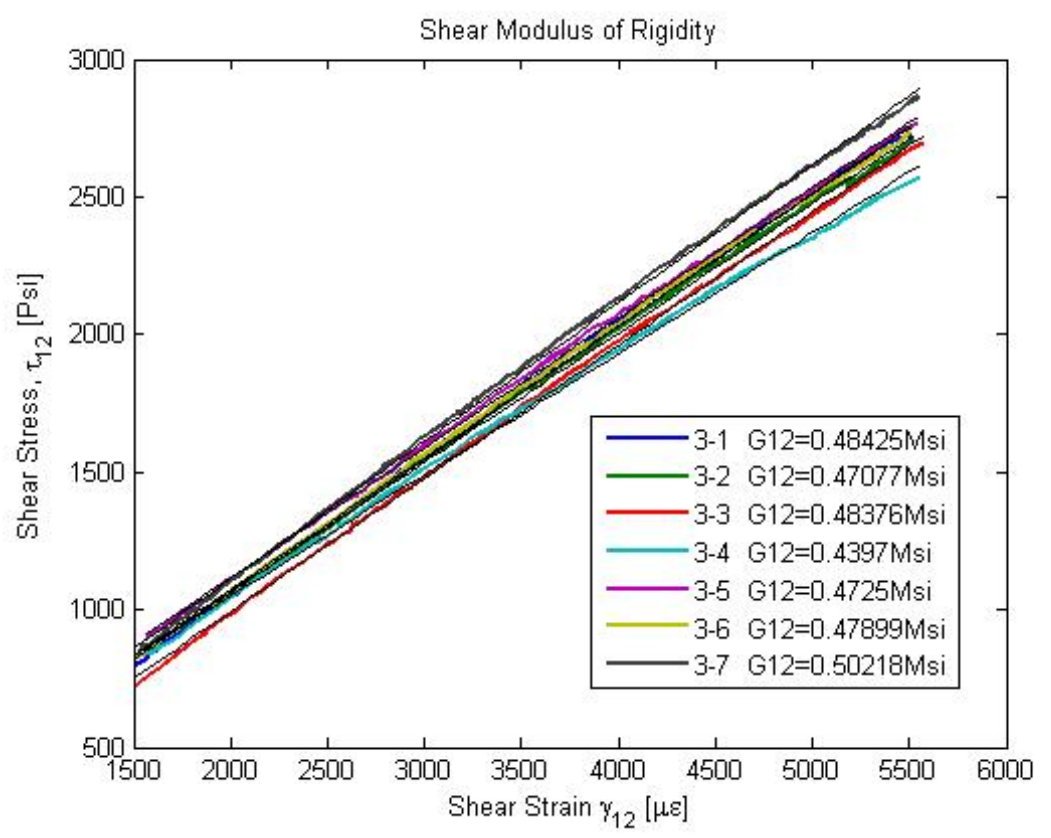

Figure 4.3: Composite in-plane shear modulus

Table 4.3: Tensile and in-plane shear statistics

\begin{tabular}{cccc}
\hline \hline Property & Mean Value & Standard Deviation & Coefficient of Variation \\
\hline$E_{1}=E_{2}$ & $8.038 \mathrm{Msi}$ & $0.141 \mathrm{Msi}$ & $1.750 \%$ \\
$\nu_{12}=\nu_{21}$ & 0.053 & 0.008 & $15.619 \%$ \\
$F_{1 t}=F_{2 t}$ & $94.03 \mathrm{Ksi}$ & $4.153 \mathrm{Ksi}$ & $4.417 \%$ \\
$\epsilon_{1 t}^{u}=\epsilon_{2 t}^{u}$ & $1.170 \%$ & $492.67 \mu \epsilon$ & $4.211 \%$ \\
$G_{12}$ & $0.476 \mathrm{Msi}$ & $0.019 \mathrm{Msi}$ & $4 \%$
\end{tabular}

unidirectional composite. This fundamental difference between woven and unidirectional composites helps to validate the data listed above. Half as many fibers over the same area causes the modulus to become more dependent on the matrix. This forces a stress-strain curve with a shallower slope and leads to a lower required failure stress for a given failure strain (about 1\%).

\subsection{Short Beam Shear Testing}

The short beam shear (SBS) test, as mentioned in Chapter 3, was used in this study as a tool to compare co-cured and post-bonded laminates. The three-point bend fixture induced a vertical shear load at the point of load application, along with a normal stress due 
to the bending moment. The beams were designed such that the shear failure would occur prior to failure due to normal stress, and only one midplane orientation was investigated.

There are three major failure modes in SBS testing that may arise: interlaminar shear, flexure, and inelastic deformation [3]. After failure, a load of 70lbf was applied to the specimens via the three-point bend fixture to induce a large enough deflection to view the damage. Photographs of each loaded specimen were taken to determine their failure modes. The specimens from the post-bonded plate failed predominantly by inelastic deformation, with $50 \%$ of the specimens falling into that category. Visual observation of the specimen under load did not reveal any cracks or other failures, the unloaded beam retained much of the deformation caused by the load. Figure 4.4 shows one example of this failure; the upper sample is an untested coupon and the lower has been tested to failure. The black, straight lines have been inserted into the image to simulate an un-deformed state. Note the permanent deformation and weave distortion in the lower sample.

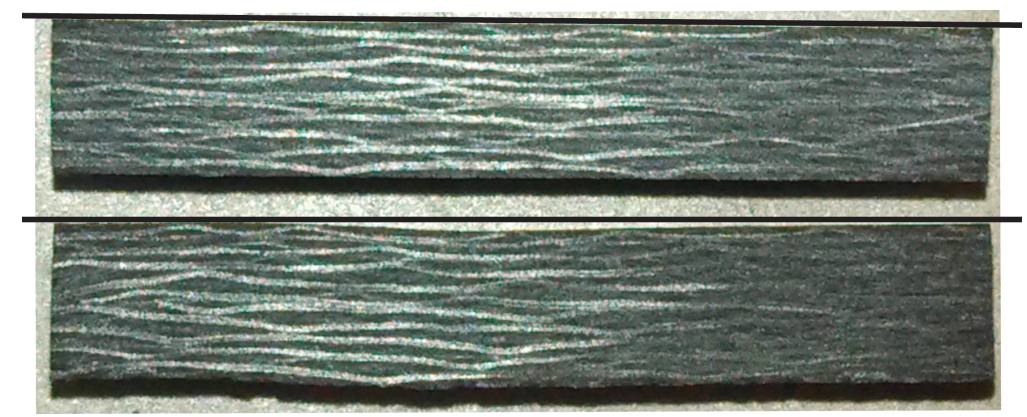

Figure 4.4: Short beam shear inelastic deformation failure mode

Further observations of specimens from plate 13 revealed two beams which failed in interlaminar shear and one that failed predominantly in flexure (both tension and compression failures) with traces of interlaminar shear failure. Plate two had four specimens which failed in interlaminar shear and two by inelastic deformation. Figures 4.5 and 4.6 show examples of flexural and interlaminar shear failure, respectively. 


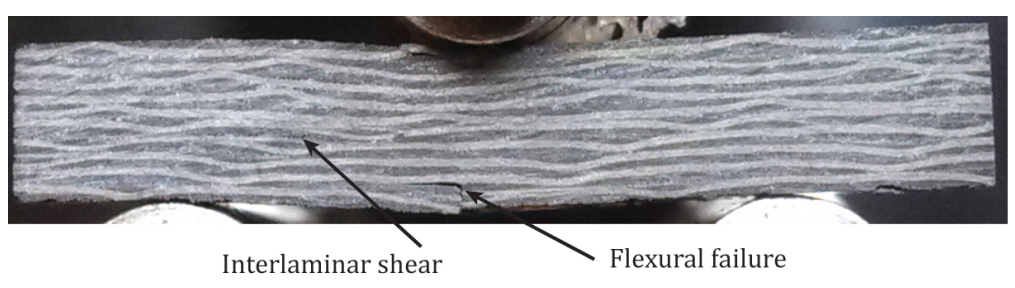

Figure 4.5: Short beam shear flexure failure mode

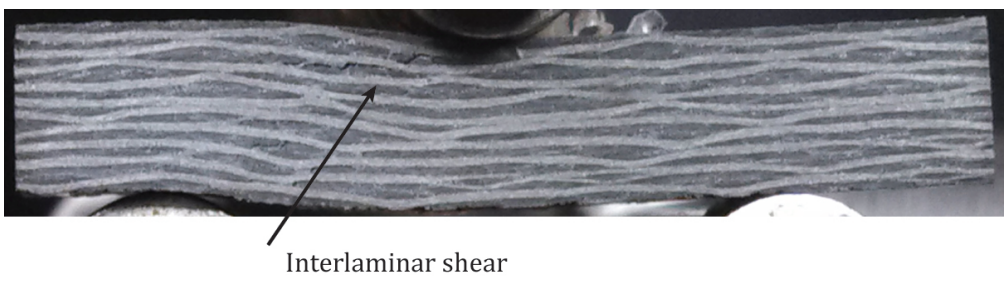

Figure 4.6: Short beam shear interlaminar shear failure mode

The results from short beam shear testing of plates 12 and 13 can be found in Table 4.4 and load-displacement plots for plate 12 and 13 specimens can be found in Figures 4.7 and 4.8, respectively. The SBS strength is 104.35Psi higher, on average, for the post-bonded plate than it is for the co-cured plate. This difference has little meaning or significance because the two averages were separated by less than a standard deviation; the SBS strength for the two laminates can, therefore, be considered equal to each other. Unfortunately, the "defect" under study in this test never witnessed any damage. The midplanes of all specimens were completely intact and undamaged. This test series did not produce any results which would lead to an adequate comparison of co-cured and post-bonded laminate short beam shear strength. A possible remedy to this problem would be to vary the dimensions of the specimens in an attempt to induce shear failure at the midplane of the laminate, or to implement the Iosipescu shear test [39], rather that the SBS test.

\subsection{Flexural Testing}

Flexural testing was used in this study to compare the co-cured and post-bonded flexural specimens. The four point bend fixture was used to induce a pure bending moment between 
Table 4.4: Short beam shear data analysis

\begin{tabular}{cccc}
\hline \hline Specimen & $F^{s b s}(\mathrm{Psi})$ & Plate Statistics & \\
\hline $12-5$ & 9157.14 & Mean (Psi) & 9179.77 \\
$12-6$ & 9163.59 & & \\
$12-7$ & 9214.21 & Standard Deviation (Psi) & 103.92 \\
$12-8$ & 9274.56 & \\
$12-9$ & 8994.98 & Coefficient of Variation & $1.1 \%$ \\
$12-10$ & 9274.12 & Mean (Psi) & 9275.42 \\
$13-5$ & 9250.15 & & \\
$13-6$ & 9450.06 & Standard Deviation (Psi) & 100.16 \\
$13-7$ & 9163.27 & \\
$13-8$ & 9256.02 & & \\
$13-9$ & 9212.17 & Coefficient of Variation & $1.1 \%$ \\
$13-10$ & 9320.83 & &
\end{tabular}

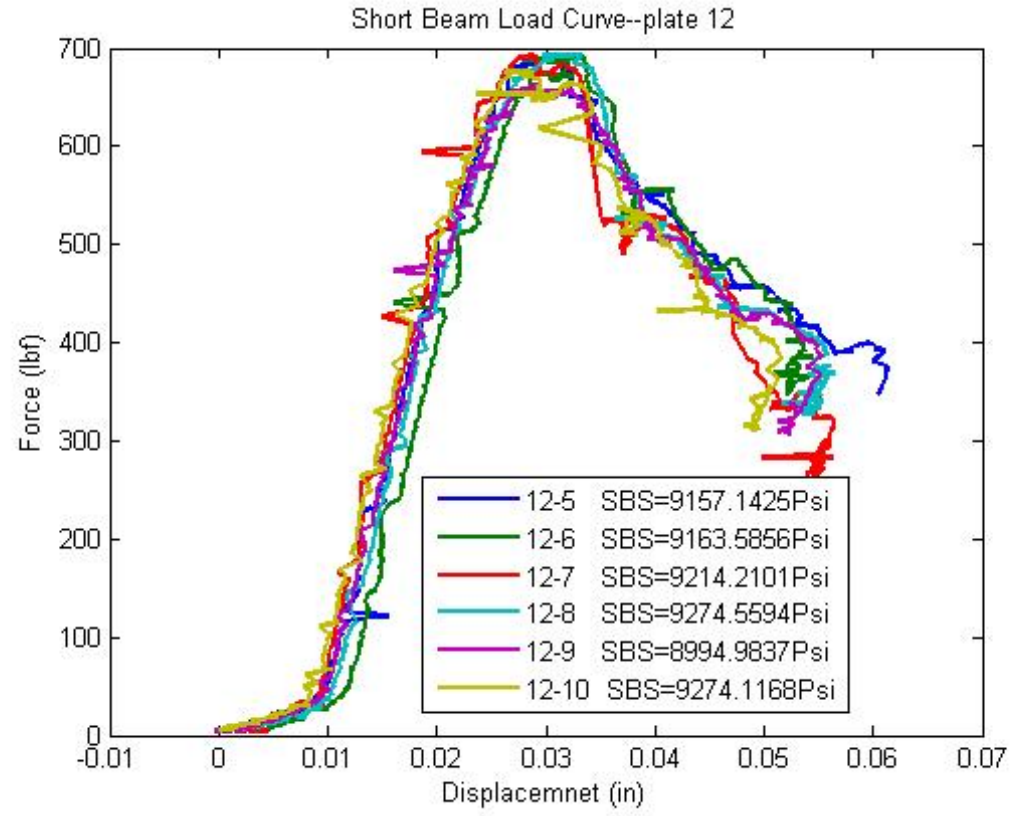

Figure 4.7: $P-\delta$ curve for plate 12 SBS specimens

the load application points. This pure moment caused a stress distribution consisting of only normal stress allowing the accurate calculation of the flexural modulus of elasticity, $E_{f}$, as mentioned in Chapter 3. Specimens from plates 12 and 13 were tested in accordance with ASTM D7264 and following the procedure listed in Section 3.5. Load and displacement were recorded and input into a MATLAB ${ }^{\circledR}$ m-file for data analysis. The load-displacement plots for plates 12 and 13 can be found in Figures 4.9 and 4.10, respectively. 


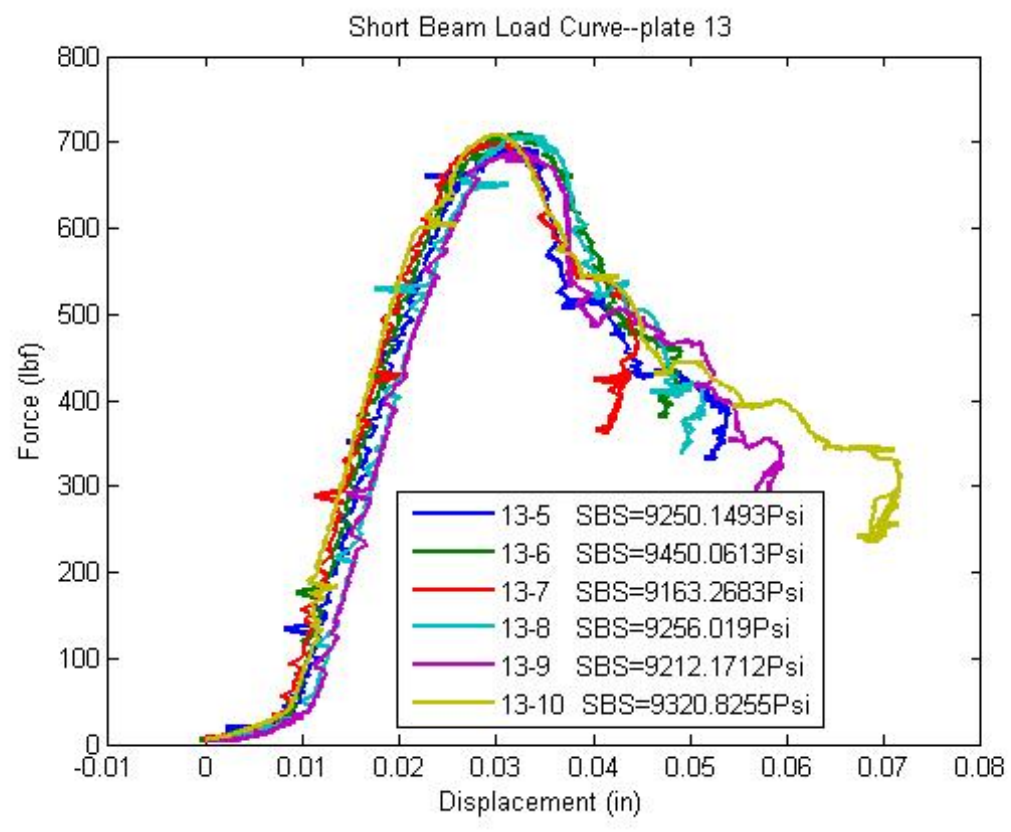

Figure 4.8: $P-\delta$ curve for plate 13 SBS specimens

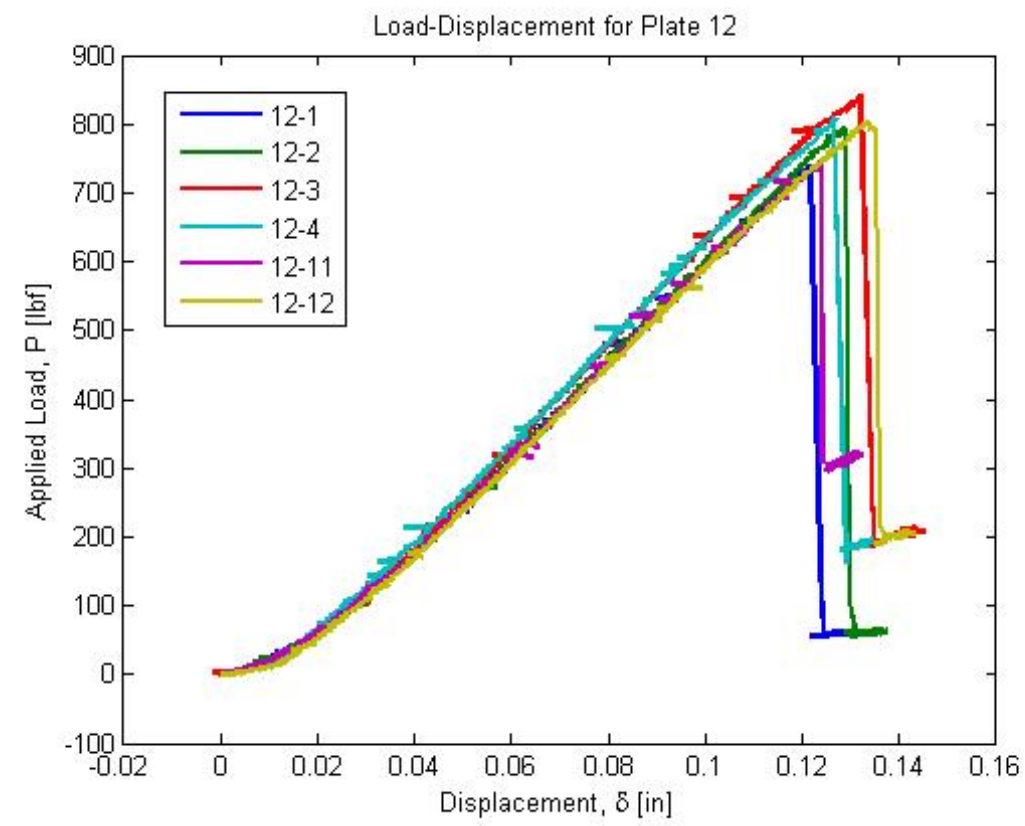

Figure 4.9: $P-\delta$ curve for plate 12 flex specimens

Differences between the co-cured and post-bonded plates may arise in the calculated value of $E_{f}$. Since, for this type of loading, stress and strain are directly related to each other through the flexural modulus, a stress-strain plot would quickly reveal any differences between the two plates. This type of comparison must be done before failure occurs, after 


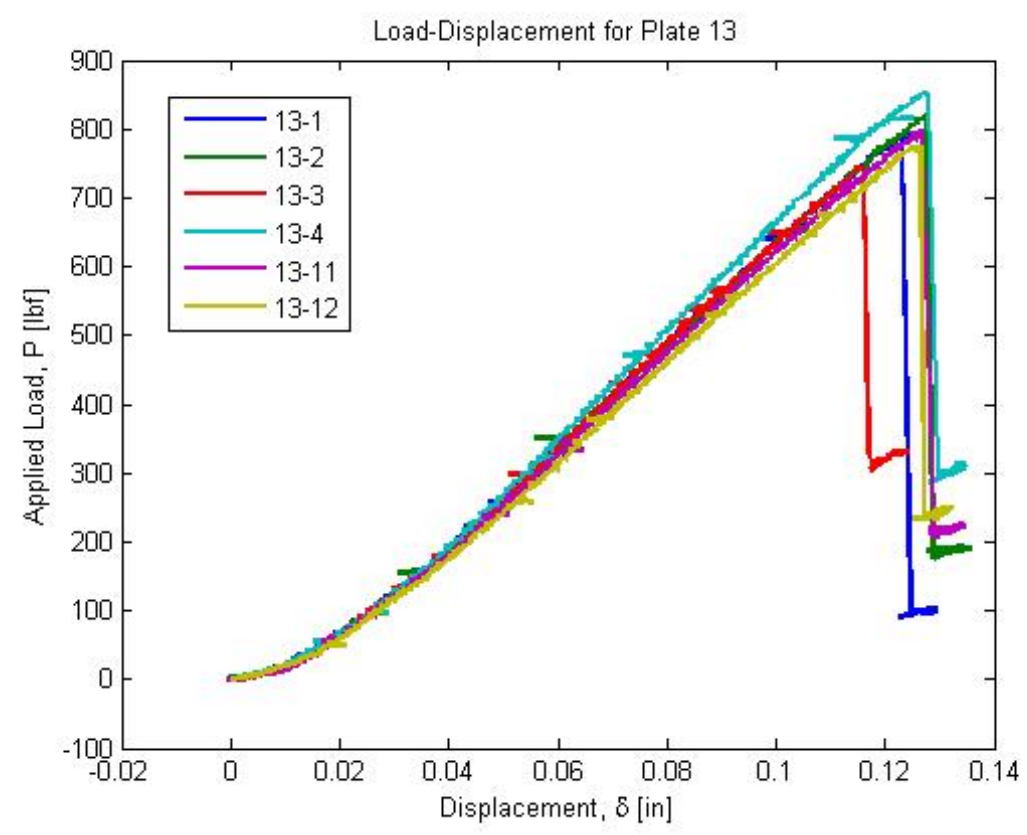

Figure 4.10: $P-\delta$ curve for plate 13 flex specimens

which time the stress and strain no longer relate linearly. Figures 4.11 and 4.12 are the stress-strain plots needed for the determination of the flexural modulus. Another pre-failure comparison between the two laminates can be made through a type of beam stiffness. Comparing the ratio of induced load to applied head displacement for each specimen from the two laminates would produce a term, not equal to the beam stiffness, which would be repeatable for all tests. The calculated "stiffness" would have to be normalized by the beam cross sectional area to alleviate any geometrical effects on the calculated value.

Table 4.5 shows the results from calculations of the flexural modulus and the statistical analysis for both plates. The co-cured plate had an average flexural modulus, $E_{f}=5.709 \mathrm{Msi}$ and the post-bonded plate had and average $E_{f}=5.687 \mathrm{Msi}$ with standard deviations of $0.373 \mathrm{Msi}$ and $0.357 \mathrm{Msi}$, respectively. The difference between these two average values is far less than a standard deviation, implying that post-bonded and co-cured laminates will have similar flexural moduli, assuming no prior damage has occurred. Comparing the ratio of induced load to applied head displacement for each specimen from the two laminates yields the results listed in Table 4.6 where $K_{B}$ is the maximum applied load divided by the 


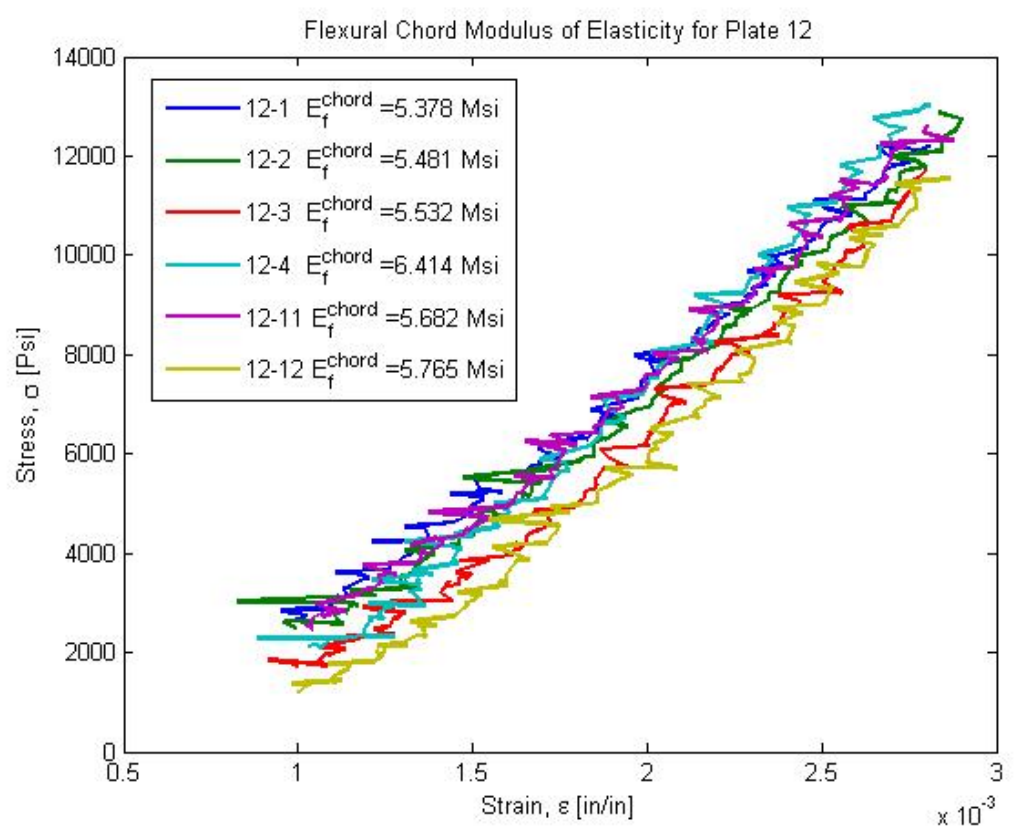

Figure 4.11: Flexural modulus for plate 12 (co-cured)

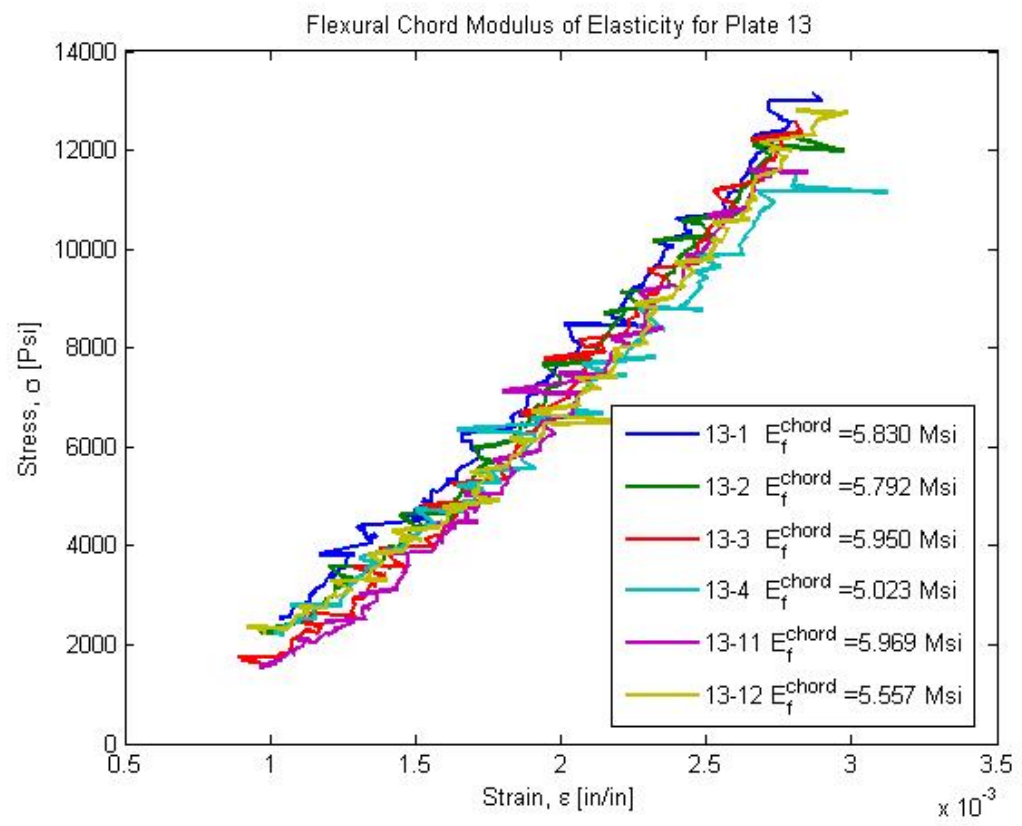

Figure 4.12: Flexural modulus for plate 13 (post-bonded)

corresponding product of cross sectional area and head deflection:

$$
K_{B}=\frac{P_{\max }}{b h \delta} .
$$


Table 4.5: Flexural modulus data analysis

\begin{tabular}{cccc}
\hline \hline Specimen & $E_{f}(\mathrm{Msi})$ & Plate Statistics & \\
\hline $12-1$ & 5.378 & Mean (Msi) & 5.709 \\
$12-2$ & 5.481 & & \\
$12-3$ & 5.532 & Standard Deviation (Msi) & 0.373 \\
$12-4$ & 6.414 & Mean (Msi) & 5.687 \\
$12-11$ & 5.682 & Coefficient of Variation & $6.5 \%$ \\
$12-12$ & 5.765 & & \\
\hline $13-1$ & 5.830 & & \\
$13-2$ & 5.792 & & \\
$13-3$ & 5.950 & Standard Deviation (Msi) & 0.357 \\
$13-4$ & 5.023 & & \\
$13-11$ & 5.969 & Coefficient of Variation & $6.3 \%$ \\
$13-12$ & 5.557 & &
\end{tabular}

It is important to note that $K_{B}$ is not a true stiffness term, it is meant only to serve as a comparison tool. The co-cured plate had an average $K_{B}=6178.5 \mathrm{Pci}$ and the post-bonded plate had an average $K_{B}=6403.3 \mathrm{Pci}$ with standard deviations of 177.4Pci and 188.7Pci, respectively. The average values of $K_{B}$ between the two plates differed by far less than a standard deviation, again indicating that a post-bonded laminate will likely behave like a co-cured laminate in bending. The two plates did have slightly different failure criteria, with maximum stresses of $101.35 \mathrm{Ksi}$ and $97.92 \mathrm{Ksi}$ for plates 12 and 13, respectively, with standard deviations of $2.75 \mathrm{Ksi}$ and $3.4 \mathrm{Ksi}$. The difference between the average failure stress for the two plates was slightly higher than a standard deviation, but still not high enough to be of significance.

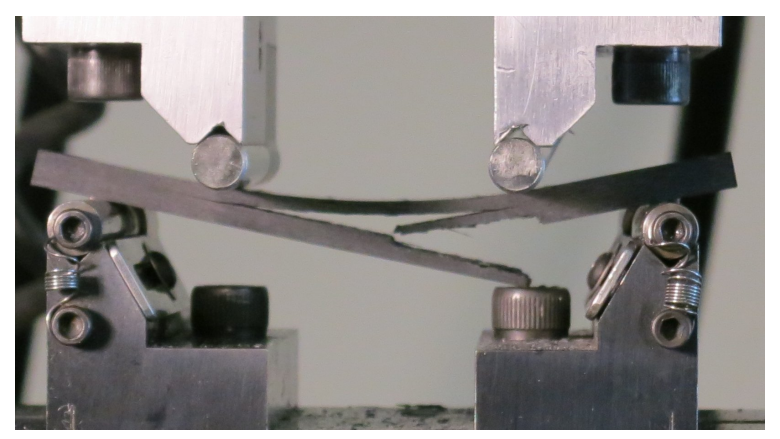

Figure 4.13: Typical failure mode for flex specimens under four point bending 
Table 4.6: Flexural modulus data analysis

\begin{tabular}{cccc}
\hline \hline Specimen & $K_{B}(\mathrm{Kci})$ & Statistics & \\
\hline $12-1$ & 68.27 & Mean (Kci) & 67.67 \\
$12-2$ & 67.32 & & \\
$12-3$ & 67.50 & Standard Deviation (Kci) & 1.1957 \\
$12-4$ & 69.59 & \\
$12-11$ & 67.40 & Coefficient of Variation & $1.8 \%$ \\
$12-12$ & 65.97 & Mean (Kci) & 68.05 \\
\hline $13-1$ & 68.91 & & \\
$13-2$ & 68.23 & & \\
$13-3$ & 68.47 & Standard Deviation (Kci) & 0.69 \\
$13-4$ & 66.90 & & \\
$13-11$ & 68.03 & Coefficient of Variation & $1.0 \%$ \\
$13-12$ & 67.76 & &
\end{tabular}

Post-failure behavior of the two laminates indicates failure of the same type for all specimens. Figure 4.13 shows the failure mode of a typical flex specimen under four point bending. Tensile failure initiated directly underneath one of the load application points. A crack then propagated from that point to another point partially through the thickness of the laminate and proceeded down the length of the beam until the load reached a minimal level (usually $75-250 \mathrm{lbf}$ ).

\subsection{DCB Testing}

DCB specimens from plates 6 - 11 were tested according to ASTM D5528, following the procedure outlined in section 3.7.3. Variations between specimens included propagation direction and delamination insertion method, which also allowed for the comparison of post-bonded to co-cured delamination interface planes. Recall from Table 3.1 that plates 6,7,8 and 11 had delaminations along fill yarns and plates 9 and 10 had delaminations along weft yarns. A 0.0005 in thick Teflon ${ }^{\circledR}$ insert was used to start the delamination in the co-cured plates (7, 9 and 11). Pending patent \#13830648 [25] was followed to insert the delamination in the post-bonded plates $(6,8$ and 10$)$.

Load, displacement, and crack length were recorded, as discussed in section 3.7.2, and input into a MATLAB ${ }^{\circledR}$ m-file for data analysis. The MATLAB ${ }^{\circledR}$ code required inputs 


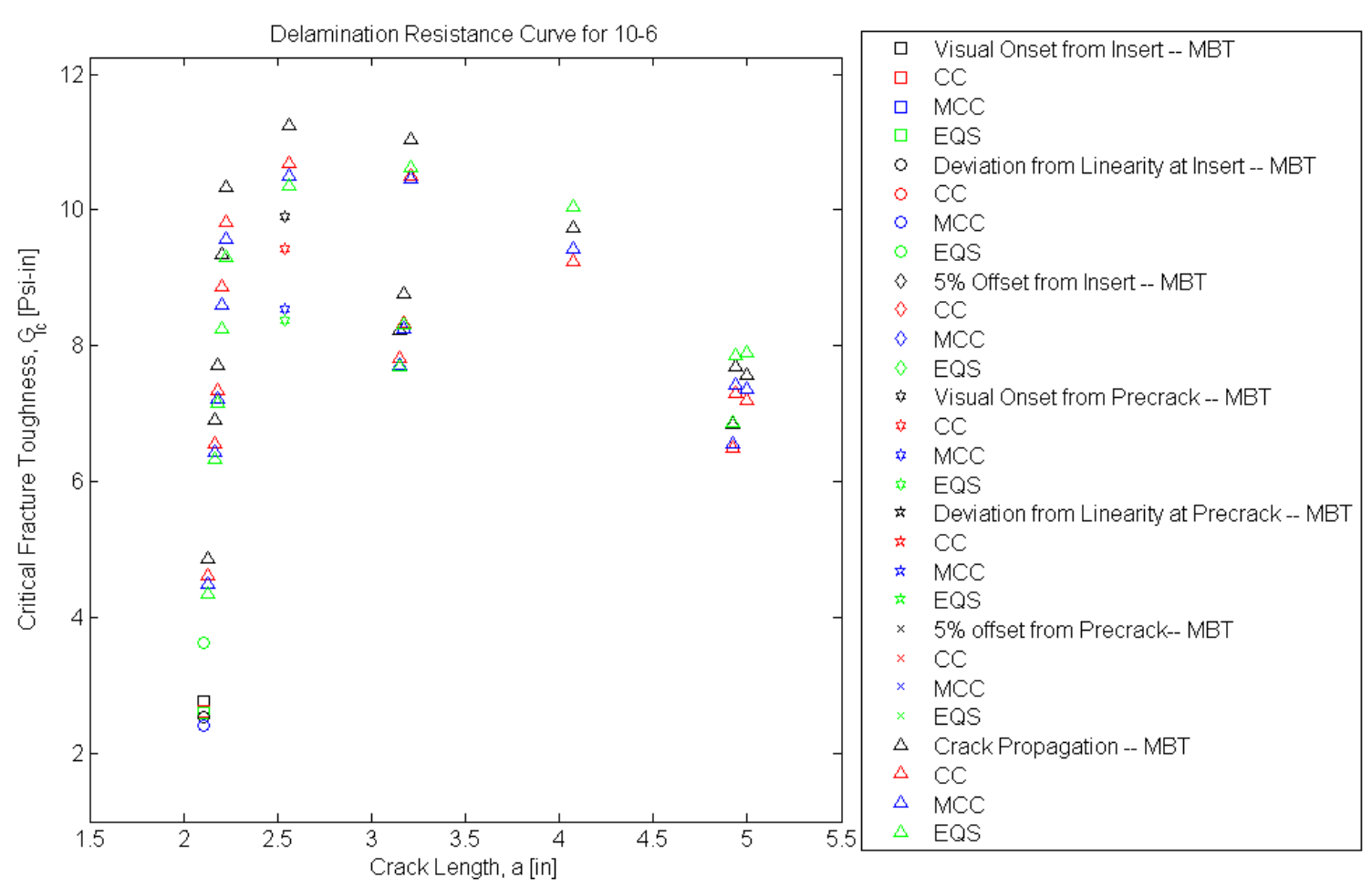

Figure 4.14: Generated R-curve for specimen $10-6$

from the LVDT, LCH-100, and manual records of crack length corresponding to specific load and displacement values. Once these values were input, outlying data points were excluded from the load and displacement data series based upon the gap between consecutive displacement and/or load points. A 0.04in difference between displacement points was chosen as the maximum allowable variation which allowed all crack propagations to be included in the data series. The allowable load variation was set to $251 b f$. The adjusted load and displacement data were matched to each crack length record and LEFM was applied through the MBT, CC, MCC, and EQS data reduction methods, as discussed in Chapter 2. Large displacement corrections were implemented where necessary and the R-curves and $P-\delta$ plots were generated. Examples of these plots for specimen $10-6$ can be found in Figures 4.14 and 4.15, respectively. R-curves, or fracture resistance curves, as seen in Figure 4.14, are plots of the calculated value of fracture toughness, $G_{I c}$, for each recorded crack length, $a$. They generally indicate the trend of increasing fracture resistance with increasing crack length until a steady state value is reached. R-curves generally only include one method for fracture toughness calculation; four data reduction methods will be included in the R-curves 
generated in this study: the EQS, MBT, CC, and MCC methods. Initiation from the insert and the precrack, along with every propagation value will be plotted.

Appendix $\mathrm{F}$ contains the $P-\delta$ plots from each test specimen and Appendix G contains the R-curves from each test specimen. Data reports, as recommended by ASTM D5528, are included in Appendix H. These reports include a statistical summary of the calculated values of $G_{I c}$ for the MBT, CC, MCC, and EQS methods corresponding to the initial crack, the precrack, and each propagation value for each plate, as well as a statistical analysis for each specimen.

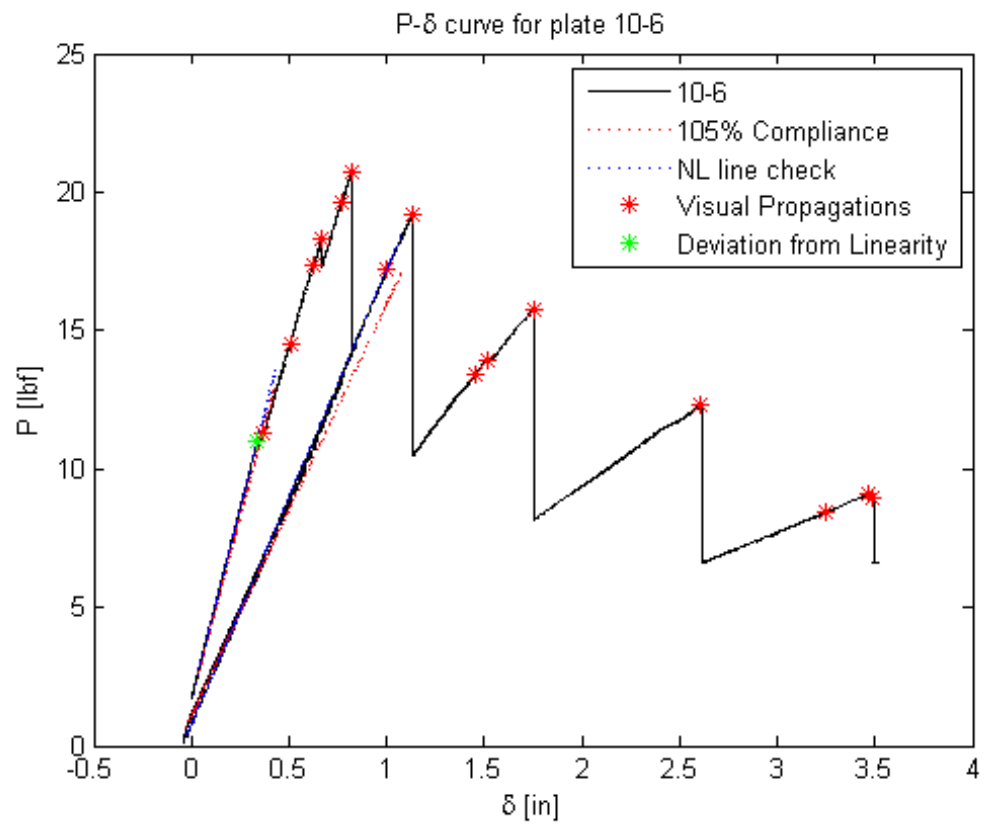

Figure 4.15: $P-\delta$ trace for specimen $10-6$

Fracture for each specimen began with the gradual opening of the crack to the location of the tip of the initial delamination, usually $63 \mathrm{~mm}$ past the end of the beam. Crack progression proceeded from this point with an increase in load and a run-arrest phenomenon which is not normally desired in DCB testing because it is caused by a build up of strain energy which, when released, allows the crack to propagate a large distance. This runarrest crack progression can often cause a crack tip to extend ten or more millimeters past its previously known position. The accurate determination of fracture toughness for the 
specific material used in this study, however, was not required. The goal of this study, as was stated in Chapter 1, was to compare post-bonded and co-cured laminates, compare the fracture toughness of fill and weft yarn delamination directions, evaluate the effectiveness of the EQS method in determining fracture toughness values similar to those found with the MBT, CC, and MCC methods, and to assess the applicability of the alternate delamination insertion method. In an effort to retain correlation, specimens were not modified in any way to avoid the run-arrest type of crack growth and $G_{I c}$ were compared on a relative basis.

Non-linearity was apparent on the $P-\delta$ plots for crack initiation at the insert for $73 \%$ of the specimens and at the precrack for $79 \%$ of the specimens which indicates failure at the center of the beams prior to visual onset at the edges. A $5 \%$ increase in compliance did not occur for any recorded crack growth in this study. Verification of the NL initiation value was made by inspecting the delamination interface plane after testing was complete. Figure 4.16 shows an example of one of the specimens with crack propagation along fill yarns. The blue lines running across the width of the beam are areas of plastic deformation of the matrix due to the large build-up of strain energy before crack propagation. The curvature of the lines indicates crack growth beginning at the interior of the beam prior to visual observation, as suggested by the NL initiation indicated on the $P-\delta$ traces.

Note: Comparisons and statistical analyses in $\S 4.4 .1, \S 4.4 .2$, and $\S 4.4 .4$ were done using the MBT, CC, and MCC data reduction methods and did not include values calculated with the EQS method. EQS values were used later in the study to assess its correlation to the other methods.

The average fracture toughness for the co-cured laminates with delaminations propagating along fill yarns was about 4Psi-in for plate 7 and 4.3Psi-in for plate 11. This correlates well with data for $5 \mathrm{H}-\mathrm{S}$ carbon cloth weaves with delaminations propagating along fill yarns tested by Nidal et al. $[14,15]$ where $G_{I c} \cong 3.7$ and 4 Psi-in. With the expectation that fracture toughness will increase with an increased weave index, the values found in this study are within reason. Comparisons to unidirectional carbon specimens with delaminations 


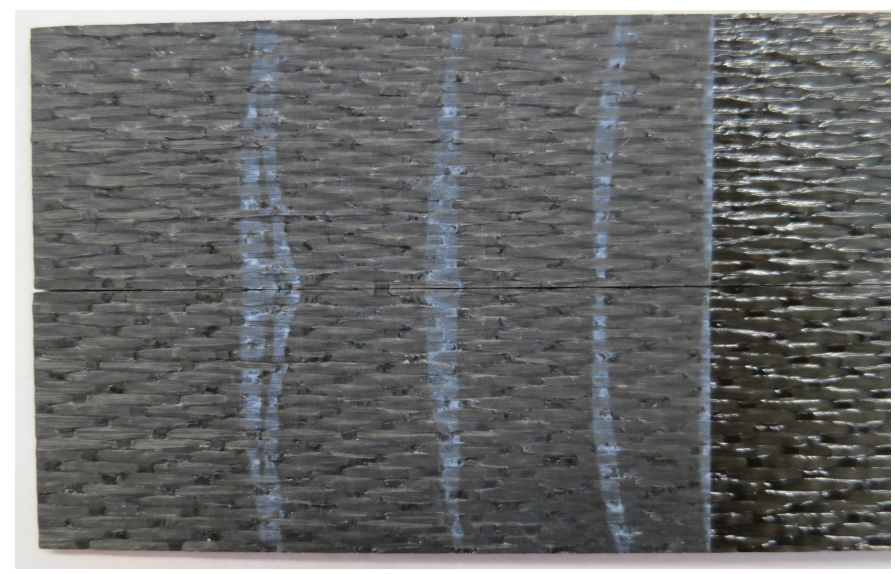

Figure 4.16: Non-linear propagation verification and could be evidence of matrix plastic deformation

propagating along the fiber direction indicates the expected higher resistance to fracture in the WFC specimens. Aliyu and Daniel [16] found $G_{I c} \cong 1.2$ Psi-in for carbon/epoxy unidirectional specimens and Robinson and Song [58] found $G_{I c} \cong 1.4$ Psi-in for unidirectional carbon/epoxy laminates.

\subsubsection{Post-Bond vs. Co-Cure Mode I Fracture}

Specimens from co-cured and post-bonded laminates, which in all other respects were identical to each other, were tested to measure how mode I fracture toughness varied with respect to the manufacturing process. Recall from Chapter 3 that plates 6,8 and 10 were laminates created through a post-bond operation and plates 7,9 and 11 were co-cured. Failure in all of the specimens with fill yarn delamination directions (plates 6,7,8 and 11) was cohesive in nature with minimal visual differences between the co-cured and post-bonded coupons. This type of failure is indicative of a good bond in the post-bonded plates. Adhesive failure, where the majority of the matrix material remains adhered to only one side of the beam, would have indicated improper bonding between the cured half-laminate and the final four layers during the post-bond operation. Without the use of a scanning electron microscope (SEM), the failure nature of the specimens with delamination propagation in the weft yarn direction is not easily determined. It is clear, after a visual examination of the two halves of the interface region, however, that the two sides behaved in a similar manner, leading to 
the conclusion that a good adherence was achieved during the post-bond operation.

Unfortunately, several problems with plate 6 led to a lower than expected fracture toughness. Plate 6 beams had lengths shorter than the minimum suggested length by ASTM D5528 and the loading hinges were originally bonded onto the wrong end of the beams. The hinges were removed by applying localized heat to break down the $3 \mathrm{M}^{\mathrm{TM}}$ adhesive bonding the hinges to the specimens. This heat application may have changed the properties of the laminate and, likewise, the values of fracture toughness calculated from experimental data. The comparison of plate 8 to plates 7 and 11, however, should yield the same results since plates 6 and 8 have the same layup schedule and were created in the same way.

Average values of $G_{I c}$, calculated using the MBT, CC, and MCC data reduction methods revealed a significantly lower resistance to fracture in the post-bonded laminates than in the co-cured laminates. Three different types of averages were used:

1. Mean propagation $G_{I c}$ values in post-bonded and co-cured plates were compared,

2. Mean Vis and NL initiation values from the precrack in post-bonded and co-cured plates were compared,

3. Mean $G_{I c}$ values calculated from similar crack lengths on each plate in post-bonded and co-cured plates were compared.

Crack initiation values from the insert were not used in the comparison between the two types of laminate because the inserts used in the co-cured plates were all Teflon ${ }^{\circledR}$ film while the post-bonded plates all utilized pending patent \#13830648 for delamination insertion. Table 4.7 shows the results from the comparison techniques listed above. Values listed indicate how much lower fracture resistance was for post-bonded plates than co-cured plates. Firm conclusions cannot be drawn from these comparisons. It is clear that postbonded laminates have a lower fracture resistance than co-cured laminates, the extent of this strength reduction, however, cannot be determined from this test series. Future studies should be made on a crack length basis utilizing only initiation values. Several post-bonded 
and co-cured plates should be fabricated, each with a different initial delamination length. Specimens from these plates should be tested to find crack initiation values, only. $G_{I c}$ corresponding to each crack length should be compared between the post-bonded and co-cured laminates. Ideally, a constant percent variation should exist between $G_{I c}$ initiation values at each crack length between the two laminate types. See $\S 5.2$ for an example test series.

Table 4.7: Comparisons between post-bonded and co-cured laminates

\begin{tabular}{ccc}
\hline \hline Comparison & $0^{\circ}$ Delamination & $90^{\circ}$ Delamination \\
\hline 1 & $10 \%$ & $4 \%$ \\
2 & $3 \%$ & $21 \%$ \\
3 & $8-22 \%$ & $2-12 \%$
\end{tabular}

Fracture resistance curves (see Appendix G) revealed similar trends between the postbonded and co-cured plates. Plates 6,8 , and $10\left(0^{\circ}\right.$ delamination $)$ were expected to have a decreased structural dependence of fracture toughness by providing fewer alternate paths for crack growth. Specimens with $90^{\circ}$ delaminations, in particular, seem to have a significant structural dependence on crack growth, as will be discussed in $\S 4.4 .2$, below, which was not changed by the use of post-bonds. Figure 4.17 shows an example of how the crack traveled around the weft yarns in plates 9 and 10. This sort of deviation from a straight line path is not ideal for the accurate calculation of fracture toughness, but it does emphasize the structural dependence of crack growth.

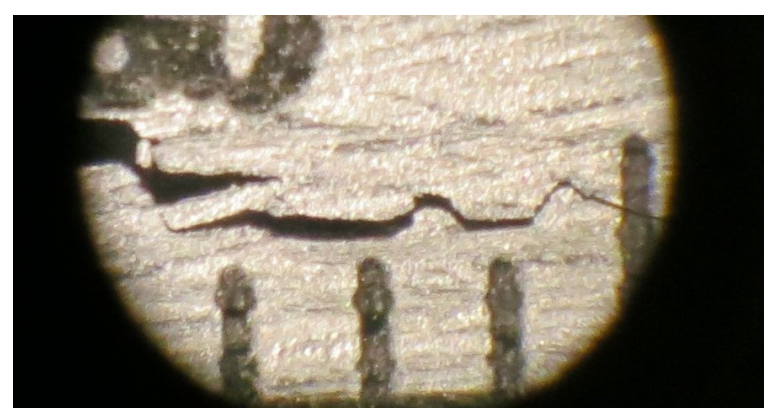

Figure 4.17: Out of plane crack growth due to the complex structure of WFCs in $90^{\circ}$ specimens 


\subsubsection{Direction Dependence for Mode I Fracture}

The dependence of fracture toughness on delamination propagation direction was investigated through testing of coupons built with delaminations set to propagate in both of the principal material directions. Recall from Chapter 3 that plates 6,7,8 and 11 had delaminations built to propagate at $0^{\circ}$ and plates 9 and 10 had delaminations built to propagate at $90^{\circ}$. As mentioned in $\S 4.4 .1$, failure in the $0^{\circ}$ specimens appeared to be mainly cohesive and failure in the $90^{\circ}$ specimens was indeterminable without the use of SEM.

Visual observation of the crack tip progression in both delamination directions suggested a larger structural dependence of fracture toughness in the $0^{\circ}$ specimens than in the $90^{\circ}$ specimens. Figures 4.17 and 4.18 are examples of typical crack tip progression in the $90^{\circ}$ and $0^{\circ}$ specimens, respectively. Notice how the crack tip, in the $90^{\circ}$ specimens frequently travels under and over the transverse yarns, producing a very jagged fracture surface and often causing transverse yarn debonding. The crack tip in the $0^{\circ}$ specimens proceeded along the fill yarns until a weft yarn was reached, at which time it could change direction (once every unit cell) and again cause transverse yarn debonding. The frequency at which this direction change could occur in the $90^{\circ}$ specimens was eight times greater than in the $0^{\circ}$ specimens and Alif et al. [15] suggested that this is the cause for the run-arrest behavior in WFCs. Visual inspection of the fracture surfaces after testing further emphasized this larger dependence on structure in the $90^{\circ}$ specimens.

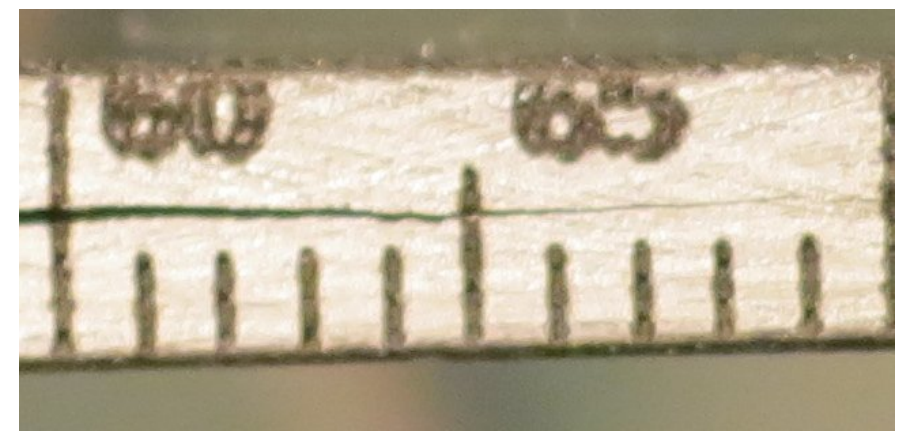

Figure 4.18: Typical crack tip progression in $0^{\circ}$ specimens 
Fracture behavior, in terms of how much energy is stored before a large fracture occurs, can be compared through the R-curves. Figure 4.19 shows a side-by-side comparison of typical R-curves for $90^{\circ}$ and $0^{\circ}$ delamination DCB specimens. The initial rise in the fracture toughness for the $90^{\circ}$ specimens was caused by the almost immediate increase in unknown crack surface area because of out of plane crack propagation. Fracture in the $90^{\circ}$ specimen occurred more frequently but the number of large fractures is about the same as for the $0^{\circ}$ specimen. The closely packed data on the left is indicative of a build up of strain energy before a larger fracture, at which point there is a break in the data until the crack opens up to its new length and the process repeats. The $0^{\circ}$ specimen does not show nearly as much energy build up.
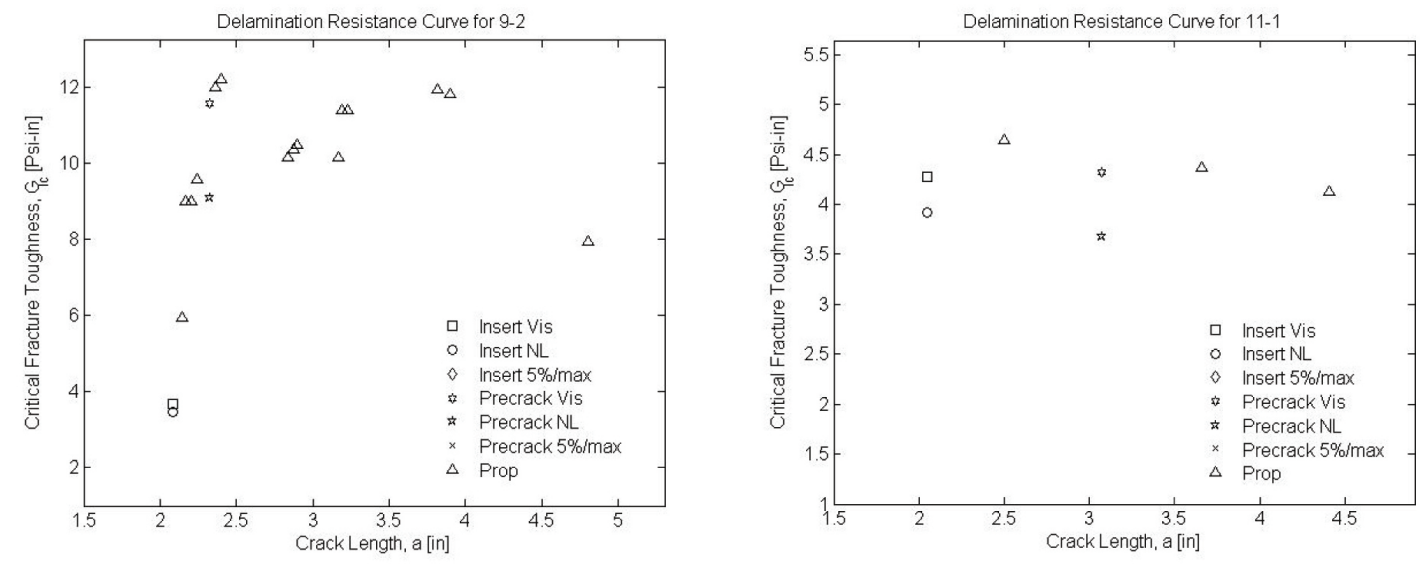

Figure 4.19: Difference between $90^{\circ}$ (left) and $0^{\circ}$ (right) R-curves

A comparison of the lowest value of $G_{I c}$ from the $90^{\circ}$ plates to the highest value of $G_{I c}$ from the $0^{\circ}$ plates revealed that the $90^{\circ}$ specimens were at least twice as able to resist fracture than the $0^{\circ}$ specimens. This comparison, however, is between a co-cured laminate with $G_{I c}=4.35$ Psi-in and a post-bonded laminate with $G_{I c}=4.352$ Psi-in $(2.04$ times larger). A comparison between only post-bonded and only co-cured laminates shows that the post-bonded, $90^{\circ}$ specimens are 2.22 times more resistant to fracture than post-bonded $0^{\circ}$ specimens. Likewise, co-cured $90^{\circ}$ specimens are 2.36 times more resistant to fracture than co-cured $0^{\circ}$ specimens. This difference cannot be contributed to material properties since the fill and weft yarns are identical and the laminates are the same except for the 
orientation of the plies. The only attribute that can change the fracture toughness between the two laminates is the material structure at the delamination interface plane. The constant path deviation of the crack tip in the $90^{\circ}$ specimens, as opposed to the minimal path deviation in the $0^{\circ}$ specimens, means that energy input from the constant displacement waveform went into creating excess crack surfaces that are not easily transformed into crack length. Recall from Chapter 2 that fracture toughness is the critical value of the strain energy release rate, and the strain energy release rate is the change in the total potential energy with an infinitesimal change in crack surface area. The crack surface area must be measured accurately in order to produce an accurate value of fracture toughness. The new crack surface areas created when the crack tip travels under or over a transverse yarn, or when transverse yarn debonding occurs, will contribute to the energy consumption of the system.

The easiest way to omit any unknown surface area in the energy term is to use the value of fracture toughness calculated from the initial propagation at the delamination insert. The problem with this is that stress concentrations often exist at the end of the insert which may artificially alter the toughness. Precracks, where the crack is propagated $3-5 \mathrm{~mm}$ past the insert, the beam is unloaded, and the test restarted, are often used to avoid this stress concentration but there is no guarantee that the surface area will be accurate. Which of these values to use, if either, is still the subject of much debate. The mean value of $G_{I c}$ for the $90^{\circ}$ and $0^{\circ}$ specimens, calculated at the insert, was $G_{I c}=3.653$ Psi-in and $G_{I c}=4.455$ Psiin, respectively, with a percent difference of $18 \%$. This result is surprising, considering the toughness is actually higher for the $0^{\circ}$ specimens than the $90^{\circ}$ specimens at the insert. Examining the fracture toughness of these specimens at the precrack, instead, yields $G_{I c}=6.529 \mathrm{Psi}$-in for the $90^{\circ}$ delaminations and $G_{I c}=4.197 \mathrm{Psi}$-in for the $0^{\circ}$ specimens with a much larger percent difference $(55.5 \%)$ between these two values. It is important to note that $G_{I c}$ for the $90^{\circ}$ specimens almost doubled while it stayed relatively the same for the $0^{\circ}$ specimens, indicating a significant amount of unaccounted for energy consumption in the $90^{\circ}$ tests between the insert and the precrack. The post-bonded plates behaved in a similar manner, with a percent difference of $1.9 \%$ between $G_{I c}$ values calculated at the insert for 
the $90^{\circ}$ and $0^{\circ}$ specimens. The percent difference, when $G_{I c}$ was calculated at the precrack increased to $67.9 \%$. These findings suggest that the fracture toughness should be calculated from the insert to avoid any effects from unknown crack surface areas, matrix deformation, and, in many other cases, energy dissipation caused by fiber breakage, pullout, and bridging.

Fracture toughness values calculated from crack initiation at the delamination insert reveal a much smaller spread between the $0^{\circ}$ and $90^{\circ}$ specimens. The observed average difference in fracture toughness values between crack initiation in the two principal material directions may not be a definite indication of an unequal fracture toughness for the two directions. Initiation values from the co-cured laminates indicate that the $90^{\circ}$ specimens had a slightly lower, rather than higher, resistance to fracture than the $0^{\circ}$ specimens. These findings, however, are inconclusive. For points past the delamination insert in both the co-cured and post-bonded laminates, the specimens with $90^{\circ}$ propagations resisted much higher loads and experienced much larger deflections before crack growth occurred than did the $0^{\circ}$ specimens. This could be an important design consideration if sub-critical damage is not an issue but it does not mean that the fracture toughness of the composite is any different in the two directions. Fracture behavior, however, does change significantly between the two directions. Characterization of crack propagation in WFC laminates cannot be fully achieved with the fracture toughness material property. More accurate characterization should include not only fracture toughness, but weave index and laminae orientation considerations as well, to account for the direction dependence of crack propagation. In other words, an empirical model of WFC fracture should include $G_{I c}, n_{g}$, and layup schedule inputs.

\subsubsection{Data Reduction and EQS Applicability}

The EQS method, as stated in $\S 2.6$, requires prior knowledge of the material's modulus of elasticity in the principal material directions (or the flexural modulus), the major and minor Poisson's ratios, and the principal shear modulus. Once these values are obtained, however, the EQS method can be utilized to calculate the mode I fracture toughness of a composite system. Load and crack length data must be collected for this data reduction 
method but the displacement of the load application point is not required. CLT is used, along with beam theory assumptions, to find an equivalent stiffness term which can be used in the compliance definition to form a new expression for the fracture toughness. This new expression, for convenience, has been repeated below. Chapter 2 should be consulted if the reader wishes to review how this expression was attained.

$$
G_{I c}=\left(\frac{P(a+|\Delta|)}{b}\right)^{2} d_{11} .
$$

Round robin testing done by O'Brien et al. on unidirectional composites [55] concluded that $G_{I c}$ from the MBT, CC, and MCC methods fell within at least $3.1 \%$ of each other. This difference, according to ASTM D5528, is not adequate to reveal any clear superiority between the three methods, although, as mentioned in Chapter 1, the MBT is the most recommended method because it produced the most conservative values for $G_{I c} 80$ percent of the time [6]. This author found it necessary to determine the spread in the data from the MBT, CC, and MCC methods for the material used in this study and use that spread as a baseline for a comparison with the EQS method. The fracture toughness values calculated from the MBT, CC, MCC methods for each crack length on each specimen, including visual initiation from the insert and the precrack, were compared and an average spread of $4.99 \%$ was calculated. "Good agreement" between EQS method and the other methods was defined three different ways:

1. $G_{I c}$ calculated using EQS fell within $5 \%$ (inclusive) of the maximum or minimum value from the MBT, CC, or MCC methods.

$(0.95 * \min (M B T, C C, M C C) \leq E Q S \leq 1.05 * \max (M B T, C C, M C C))$

2. $G_{I c}$ calculated using EQS fell within $5 \%$ (inclusive) of the mean value from the MBT, $\mathrm{CC}$, and MCC methods.

$(0.95 * \operatorname{mean}(M B T, C C, M C C) \leq E Q S \leq 1.05 *$ mean $(M B T, C C, M C C))$

3. $G_{I c}$ calculated using EQS differed by no more than $5 \%$ from any other value from the MBT, CC, or MCC methods.

$(0.95 * \max (M B T, C C, M C C) \geq E Q S \geq 1.05 * \min (M B T, C C, M C C))$

Values calculated by the EQS method were highly dependent on the thickness of the beams. A single average ply thickness could not be used in the CLT code to calculate the 
equivalent stiffness. Instead, average ply thickness was calculated for each specimen by dividing the specimen thickness by the number of plies in the laminate. The modulus of elasticity of the composite also had a large effect on $G_{I c}$. Meticulous care in the determination of the longitudinal and transverse moduli of elasticity (or the flexural modulus) is absolutely necessary to achieve accurate values of $G_{I c}$ from the EQS method. The longitudinal/transverse modulus was used for all values of EQS $G_{I c}$ in this study. Definition 1, above, indicated that the EQS method had good agreement with the other three methods $77 \%$ of the time, definition 2 indicated good agreement for $71.4 \%$ of the calculations, and definition 3 implied good agreement for $63.4 \%$ of the recorded crack propagations. The EQS method calculated $G_{I c}$ conservatively (lower than all of the other values) for over $33 \%$ of the propagations. Since definition 3 produced the lowest percentage of propagations for which the EQS method was adequate, it was utilized for the evaluation of the applicability of the EQS method. $63.4 \%$ agreement is not sufficient for the accurate measurement of a system's mode I fracture toughness. The EQS method should, therefore, be avoided if sufficient testing equipment is available to measure, accurately, the load and displacement at the load application point of the DCB specimens. If the displacement cannot be accurately measured, the EQS method may be considered.

An additional attempt at attaining good agreement was made by changing one of the assumptions made in the formulation of the equivalent stiffness, $\overline{E I}$, term. In $\S 2.6$ that anticlastic bending was allowed in the beam which allowed the line moments $M_{y}$ and $M_{x y}$ to be set to zero. If the assumption is changes to disallow anticlastic bending, the $M_{y}$ and $M_{x y}$ line moments cannot be set to zero, but the curvatures $\kappa_{y}$ and $\kappa x y$ can. This assumption changes the definition of the equivalent stiffness to

$$
\overline{E I}=b D 11
$$

and changes the definition of the EQS fracture toughness to

$$
G_{I c}=\frac{1}{D 11}\left(\frac{P(a+|\Delta|)}{b}\right)^{2}
$$


This new definition of fracture toughness lowered the EQS fracture toughness by only $0.28 \%$. Due to this insignificant change in fracture toughness, the original assumption will be maintained and EQS fracture toughness will remain defined by Equation 2.47.

\subsubsection{Applicability of Alternate Delamination Insertion Method}

Two different delamination insertion methods were used in this study: the standard method of inserting a 0.0005 in thick Teflon ${ }^{\circledR}$ film in between two lamina, and by following pending patent \#13830648 (hereafter referred to as the alternate method) which, effectively, allows for the insertion of a zero thickness initial delamination. Three plates $(7,9$, and 11$)$ were fabricated with the Teflon ${ }^{\circledR}$, and three plates $(6,8$, and 10) had delaminations inserted using the alternate method. The most considerable disadvantage of the alternate method is that it required the laminate to be made in a two step process, meaning it must contain a post-bond at the plane where the delamination is desired. All of the post-bonded plates, therefore, had delaminations inserted using the alternate method and all of the co-cured plates had delaminations inserted using a Teflon ${ }^{\circledR}$ film. Future studies on this subject should include post-bonded DCB specimens utilizing the Teflon ${ }^{\circledR}$ film delamination insertion method, as well, for a more complete comparison between the two techniques.

The insertion methods can be compared through an examination of the $P-\delta$ curve up to the first crack propagation and through a comparison of Vis, NL, and, if applicable, $5 \% /$ Max initiation $G_{I c}$ values attained from both types of inserts. If noticeable differences exist in the behavior of the $P-\delta$ curve directly before crack initiation, it may indicate that the alternate method behaves in a significantly different manner than the Teflon ${ }^{\circledR}$. Likewise, if there is a consistent discrepancy between the percent variation of the initiation values at the insert between the co-cured and post-bonded plates, a distinction between the behavior of the alternate method and the Teflon ${ }^{\circledR}$ film would be indicated. Recall that the $P-\delta$ curves for all of the test specimens can be found in Appendix $\mathrm{F}$ and the data sheets can be found in Appendix H. 
Table 4.8: Non-linear crack initiation for traditional and alternate delamination insertion methods

\begin{tabular}{cccc}
\hline \hline Plate & Laminate Type & \% NL Occurance & \% NL i Vis \\
\hline 8 & $0^{\circ}$, alternate & $33 \%$ & $4.5 \%$ \\
$7 / 11$ & $0^{\circ}$, Teflon ${ }^{\circledR}$ & $91 \%$ & $10.6 \%$ \\
10 & $90^{\circ}$, alternate & $50 \%$ & $5.8 \%$ \\
9 & $90^{\circ}$, Teflon ${ }^{\circledR}$ & $100 \%$ & $6 \%$
\end{tabular}

Comparisons were made between plates 7,11 and 8 ( $0^{\circ}$ delaminations $)$, and plates 9 and 10 (90 delaminations). Recall from $\S 4.4 .1$ that plate 6 was omitted from comparison due to possible damage during initial testing. Visual inspections of the load-displacement plots did not reveal any significant distinctions between initiation values for the two types of insert. A consistent basis for a mathematical comparison was difficult because a postbonded plate with a Teflon ${ }^{\circledR}$ delamination insert was not made. Attempts to compare the variation in initiation values between the two inserts in order to find a percent difference in Vis initiation and compare it to the predicated variation between post-bonded and co-cured plates were made. Unfortunately, a strict model for comparison between post-bonds and co-cures was not achieved in this study and initiation variations could not be compared with any meaningful outcome. Another attempt at comparison consisted of determining whether either one of the delamination insertion methods caused an increase in non-linear behavior. Table 4.8 shows the results of an examination of the non-linear behavior for each insert's crack initiation. These results lead to the conclusion that the alternate delamination insertion method allows for a decrease in not only the frequency at which specimens are subject to non-linear behavior, but also in the severity of the discrepancy between the NL and Vis $G_{I c}$ initiation values. 


\section{Chapter5}

\section{Conclusion}

\subsection{Conclusions}

Mode I interlaminar fracture of 3k 8-Harness-Satin Carbon cloth, with identical fill and weft yarns, pre-impregnated with Newport 307 resin was investigated through the DCB test (ASTM D5528 [6]). The effects of delamination interface plane bond type, crack propagation direction, and delamination insertion method were examined. The Modified Beam Theory, Compliance Calibration method, and Modified Compliance Calibration method were used for comparative purposes for these investigations and to evaluate the validity of the proposed Equivalent Stiffness (EQS) method.

Two types of delamination interface plane bonds were studied: post-bonded (co-bonded) and co-cured. The post-bonded laminates were created in a two step process: the first half of the laminate was fully cured and the second half was cured on top of the first without the application of an additional adhesive. Several attempts were made to compare the relative fracture resistance of the two types of laminate with the conclusion that a post-bonded laminate will have a lower resistance to crack propagation than a identical co-cured laminate. The severity of the discrepancy between the two types of laminate was not consistent between the comparisons so a discrete mathematical comparison between the two laminates was not attained. See $\S 5.2$, below, for future study suggestions. Post-bond effects on the flexural modulus and short beam shear strength for the 8-HS/307 material were also investigated. Significant differences between the two bond types were not witnessed. 
Crack propagation direction-dependence of fracture toughness was investigated for two delamination directions: along fill yarns $\left(0^{\circ}\right)$ and along weft yarns $\left(90^{\circ}\right)$. Delamination interface planes were fabricated to ensure mirror symmetry and the DCB specimens were fabricated with symmetry about the interface plane and about the midplane of each half of the laminate. Visual inspection of the interface plane suggested a greater structural effect on crack growth direction in the $90^{\circ}$ laminates. Unadjusted $G_{I c}$ propagation values indicated that cracks in the $0^{\circ}$ direction were $100 \%$ more susceptible to fracture than cracks in the $90^{\circ}$ direction. Additional inspection of the interface plane and comparisons of initiation values at the insert and the precrack revealed excessive energy dissipation in the $90^{\circ}$ specimens due to out-of-plane crack growth, transverse yarn debonding, and matrix deformation, which was unaccounted for the in $G_{I c}$ calculations. These energy dissipation terms did not take effect until the crack extended past the insert, so initiation values from the initial delamination were compared to reveal an $18 \%$ higher resistance to fracture in the $0^{\circ}$, rather than the $90^{\circ}$, laminates. This conclusion warrants further study, which will be outlined in $\S 5.2$, and suggests that the characterization of crack propagation in WFC laminates cannot be fully achieved with the fracture toughness material property. More accurate characterization should include not only fracture toughness, but weave index and laminae orientation considerations as well, to account for the direction dependence of crack propagation.

The applicability of a patent pending delamination insertion method has been preliminarily evaluated through a comparison with the standard method for initial delamination insertion. Half of the DCB specimens tested in this study utilized the standard 0.0005in thick Teflon ${ }^{\circledR}$ film. Delaminations were built into the other laminates through the method outlined in patent pending \#13830648. Variations between the initiation values calculated for the two insert types could not be directly contributed to differences between the two methods because the alternate method required the use of a post-bond and a firm comparison between co-cures and post-bonds was not achieved. An advantage of the alternate delamination insertion method was revealed, however, when non-linear delamination initiation effects were examined. Overall, the alternate method provided initial delaminations 
which were less susceptible to non-linear crack initiation effects. When non-linear effects were prevalent, the corresponding $G_{I c}$ initiation values differed by only $5.15 \%$ from the visual onset $G_{I c}$, as opposed to the average difference of $8.3 \%$ for the Teflon ${ }^{\circledR}$ insert. If a consistent comparison between post-bonded and co-cured laminates is achieved, the alternate delamination insertion method can be used as long as the strength reduction is accounted for. A suggestion for future study of this delamination insertion method is outlined in $\S 5.2$.

The Equivalent Stiffness method, an alternate approach for the calculation of mode I fracture toughness, was proposed. This approach used Composite Laminate Theory (CLT) to calculate an equivalent beam stiffness which was then used, along with the standard linear elastic fracture mechanics formulation for the strain energy release rate, to form a fracture toughness definition based only on specimen dimensions, material properties, applied load, and crack length. Values calculated by this method were found to be highly dependent on the thickness of the beams and the moduli of elasticity used in CLT calculations. The EQS method calculated values within $5 \%$ of the other three data reduction methods for $63 \%$ of the recorded visual propagation values (including propagation from the insert and the precrack) with over $33 \%$ of the $G_{I c}$ values from EQS calculated conservatively, compared to the other three methods. This frequency of "good agreement" is unreliable and the EQS method should not be used unless completely accurate values for the longitudinal and transverse modulus of elasticity (or the flexural modulus) are known and the available testing equipment cannot precisely measure the displacement at the load application point, as required for the other three data reduction methods.

\subsection{Future Studies}

1. A more accurate comparison of post-bonded and co-cured laminates can be attained through the fabrication of several post-bonded and co-cured plates, each with a different initial delamination length. The delaminations should be input using the Teflon ${ }^{\circledR}$ 
film method to avoid discrepancies between insert types. Specimens from these plates should be tested to find crack initiation values, only. $G_{I c}$ corresponding to each crack length should be compared between the post-bonded and co-cured laminates. Ideally, a constant percent variation should exist between $G_{I c}$ initiation values for each crack length between the two laminate types.

Table 5.1: Example test series for post-bond/co-cure comparisons

\begin{tabular}{cc}
\hline \hline Laminate & Initial Delamination Lengths \\
\hline Post-Bond & $50,100,150,200 \mathrm{~mm}$ \\
Co-Cure & $50,100,150,200 \mathrm{~mm}$
\end{tabular}

2. The direction dependence of fracture toughness can be studied further through a series of tests similar to the suggestion, above. Several $0^{\circ}$ and $90^{\circ}$ plates should be fabricated, each with different initial delamination lengths. The initiation values should be compared between the two delamination directions for each crack length. Another option would be to implement the edge delamination technique outlined in [58].

Table 5.2: Example test series for delamination direction comparisons

\begin{tabular}{cc}
\hline \hline Delamination Direction & Initial Delamination Lengths \\
\hline $0^{\circ}$ & $50,100,150,200 \mathrm{~mm}$ \\
$90^{\circ}$ & $50,100,150,200 \mathrm{~mm}$
\end{tabular}

3. A more accurate comparison of delamination insertion methods could be made through the testing of post-bonded plates containing both types of delamination inserts. This would alleviate any discrepancy due to co-curing vs. post-bonding that were witnessed in this study.

Table 5.3: Example test series for delamination insertion comparisons

\begin{tabular}{cc}
\hline \hline Laminate & Initial Delamination Lengths \\
\hline Post-bond, alternate & $50,100,150,200 \mathrm{~mm}$ \\
Post-bond, Teflon ${ }^{\circledR}$ & $50,100,150,200 \mathrm{~mm}$
\end{tabular}


4. Calibration of the Virtual Crack Closure Technique (VCCT) and Cohisive Element in Abaqus could be attempted using the data presented in Appendix H. Fracture toughness values should be input as curve fits to the R-curves rather than steady state values to account for crack initiation and the rise in energy dissipation effects as crack length increases. 


\section{BIBLIOGRAPHY}

[1] "Experimental characterization of delamination fracture". In N.J. Pagano, editor, Interlaminar Response of Composite Materials, volume 5 of Composite Materials. Elsevier Science Publishers, Amsterdam, The Netherlands, 1989. Pipes, R.b. (series ed.).

[2] Mil-hdbk-17-1f. In Department of Defense Handbook, volume 1, chapter 6, pages 128133. 2002.

[3] ASTM Standard D2344 (2006). "Standard Test Method for Short Beam Strength of Polymer Matrix Composite Materials and Their Laminates". ASTM International, West Conshohocken, PA, 2006, DOI: 10.1520/D2344_D2344M-00R06. www.astm.org.

[4] ASTM Standard D6671-06 (2006). "Standard Test Method for Mixed Mode I-Mode II Interlaminar Fracture Toughness of Unidirectional Fiber Reinforced Polymer Matrix Composites". ASTM International, West Conshohocken, PA, 2006, DOI: 10.1520/D6671. www.astm.org.

[5] ASTM Standard D3518 (2007). "Standard Test Method for Tensile Properties of Polymer Matrix Composite Materials". ASTM International, West Conshohocken, PA, 2008, DOI: 10.1520/D3039_D3039M-08. www.astm.org.

[6] ASTM Standard D5528-01 (2007). "Standard Test Method for Mode I Interlaminar Fracture Toughness of Unidirectional Fiber-Reinforced Polymer Matrix Composites". ASTM International, West Conshohocken, PA, 2007, DOI: 10.1520/D552801R07E03. www.astm.org.

[7] ASTM Standard D7264 (2007). "Standard Test Method for Flexural Properties of Polymer Matrix Composite Materials". ASTM International, West Conshohocken, PA, 2007, DOI: 10.1520/D7264_D7264M-07. www.astm.org.

[8] ASTM Standard D3039 (2008). "Standard Test Method for In-Plane Shear Response of Polymer Matrix Composite Materials by Tensile Test of a $\pm^{\circ}$ Laminate". ASTM International, West Conshohocken, PA, 2007, DOI: 10.1520/D3518_D3518M-94R07. www.astm.org.

[9] ASTM Standard D3163-01 (2008). "Standard Test Method for Determining Strength of Adhesively Bonded Rigid Plastic Lap-Shear Joints in Shear by Tension Loading". ASTM International, West Conshohocken, PA, 2008, DOI:10.1520/D3163-01R08. www.astm.org. 
[10] ASTM Standard D5868-01 (2008). "Standard Test Method for Lap Shear Adhesion for Fiber Reinforced Plastic (FRP) Bonding". ASTM International, West Conshohocken, PA, 2008, DOI: 10.1520/D5868-01R08. www.astm.org.

[11] ASTM Standard D1002-10 (2010). "Standeard Test Method for Apparent Shear Strength of Single-Lap-Joint Adhesively Bonded Metal Specimens by Tension Loading (Metal-to-Metal)". ASTM International, West Conshohocken, PA, 2010, DOI: 10.1528/D1002-10. www.astm.org.

[12] ASTM Standard D3164-03 (2011). "Standard Test Method for Strength Properties of Adhesively Bonded Plastic Lap-Shear Sandwich Joints in Shear by Tension Loading". ASTM International, West Conshohocken, PA, 2011, DOI: 10.1520/D3164-03R11. www.astm.org.

[13] Bhagwan D. Agarwal, Lawrence J. Broutman, and K. Chandrashekhara. Analysis and Performance of Fiber Composites. John Wiley and Sons, Inc., New Jersey, third edition, 2006.

[14] N. Alif, L.A. Carlsson, and L. Boogh. "The effect of weave pattern and crack propagation direction on mode I delamination resistance of woven glass and carbon composites". Composites Part B: Engineering, 29:603-611, 1998.

[15] N. Alif, L.A. Carlsson, and J.W. Gillespie. "Mode I, Mode II, and Mixed Mode Interlaminar Fracture of Woven Fabric Carbon/Epoxy". In Composite Materials: Testing and Design, volume 13, pages 82-106. 1997.

[16] A.A. Aliyu and I.M. Daniel. "Effects of Strain Rate on Delamination Fracture Toughness of Graphite/Epoxy". In Ed. W.S. Johnson, editor, Delamination and Debonding of Materials, pages 336-348, Philadelphia, 1985. American Society for Testing and Materials, ASTM STP 876.

[17] J. Andersons and M König. "Dependence of fracture toughness of composite laminates on interface ply orientations and delamination growth direction". Composites Science and Technology, pages 2139-2152, 2004.

[18] W.D. Bascom, R.J. Bitner, R.J. Moulton, and A.R. Sieber. "The Interlaminar Fracture of Organic-Matrix Woven Reinforced Composites". Composites, 11:9, 1980.

[19] J.P. Berry. "Determination of Fracture Energies by the Cleavage Technique". Journal of Applied Physics, 34(1):62-68, 1963.

[20] V.V. Bolotin, K.S. Bolotina, V.P. Radin, and V.N. Shchugorev. "Fracture Toughness Characteristics of Laminated Composites". Mechanics of Composite Materials, 32(1):14, 1996.

[21] W.L. Bradley, C.R. Corleto, and D.P. Goetz. "Fracture Physics of Delamination in Composite Materials". AFOSR-TR-88-0020, 1987.

[22] P.J. Burchill and G.J. Simpson. "Improved Interlaminar Fracture Toughness for Vinyl Ester Resin-Fibre Glass Composites". Proc ICCM, 2:254-262, 1997. 
[23] R.D.S.G Campilho, D.C. Moura, J.F.M.G da Silva, M.D. Banea, and L.F.M da Silva. "Fracture toughness determination of adhesive and co-cured joints in natural fibre composites". Composites: Part B, 50:120-126, 2013.

[24] H. Chai. "The Characterization of Mode I Delamination Frature in Non-Woven Multidirectional Laminates". Composites, 15:277-290, 1984.

[25] J.C. Chen, L.E Gomez, and J.D. Mello. Designed defects in laminate composites. Technical Report Application \# 13830648, U.S. Patent Pending, 2013.

[26] NASA CR-2218, editor. Analysis and Design of Advanced Composite Bonded Joints. National Aeronautics and Space Administrations, 1974.

[27] P. Davies, B.R.K. Blackman, and A.J. Brunner. "Standard Test Methods for Delamination Resistance of Composite Materials: Current Status". Applied Composite Materials. Kluwer Academic Publishers, Netherlands, 1998.

[28] P. Davies and O. Brunellière. J. Mat. Sci. Letters, 12:427, 1992.

[29] P. Davies, W.J. Cantwell, and H.H. Kausch. Comp. Sci. \& Tech, 35:301, 1989.

[30] P. Davies, C. Moulin, H.H. Kausch, and M. Fischer. Comp. Sci. E Tech, 39:193, 1990.

[31] Wei Ding. Delamination Analysis of Composite Laminates. PhD thesis, University of Toronto, 1999.

[32] R.F. Gibson. Principles of composite material mechanics. CRC Press, Taylor \& Francis Group, Boca Ration, FL, second edition, 2007.

[33] A.A. Griffith. "The Theory of Rupture". In Sr. J. Waltman, editor, Proc. 1st Int. Cong. Applied Mechanics, pages 55-63, Delft, 1924.

[34] S. Hashemi, A. J. Kinlock, and J G. Williams. "Corrections needed in double-cantilever beam tests for assessing the interlaminar failure of fibre-composites". Journal of Materials Science Letters, 8(2):125-129, 1989.

[35] M. Hojo, K. Kageyama, and K. Tanaka. Composites, 26(4):243, 1995.

[36] M.J. Hoke. Adhesive bonding of composites. Slide Show Presentation.

[37] J.H. Hwang, C.C. Lee, and W. Hwang. "Effect of crack propagation directions on the interlaminar fracture toughness of carbon/epoxy composite materials". Appl Compos Mater, 8:411-433, 2001.

[38] M.W. Hyer. Stress Analysis of Fiber-Reinforced Composite Materials. McGraw-Hill, 1998.

[39] N. Iosipescu. "New Accurate Procedure for Single Shear Testing of Metals.

[40] G. R. Irwin. "Analysis of Stresses and Strains Near the End of a Crack Traversing a Plate". Journal of Applied Mechanics, 24:361-364, 1957.

[41] G.R. Irwin. "Fracture". In S. Flugge, editor, Encyclopedia of Physics, volume VI, pages 551-590. Springer, Berlin, 1958. 
[42] K. Kageyama and M. Hojo. "Proposed Methods for Interlaminar Fracture Toughness Tests of Composite Laminates". Proceedings of the 5th U.S./Japan Conference on Composite Materials, pages 227-234, 1990.

[43] M.F. Kanninen. "An Augmented Double Cantilever Beam Model for Studying Crack Propagation and Arrest". International Journal of Fracture, 9:83-92, 1973.

[44] M.F. Kanninen. "A dynamic analysis of unstable crack propagation and arrest in the DCB test specimen". International Journal of Fracture, 10:415-430, 1974.

[45] P.E. Keary, L.B. Llcewicz, C. Shaar, and J. Trostle. "Mode I Interlaminar Fracture Toughness of Composites Using Slender Double Cantilevered Beam Specimens". Journal of Composite Materials, 19:154-177, 1985.

[46] S. Mall and W.S. Johnson. "Characterization of Mode I and Mixed-Mode Failure of Adhesive Bonds Between Composite Adherends". Composite Materials: Testing and Design (Seventh Conference), pages 322-334, 1986.

[47] G. Marom, R.H. Harel, M. Rosensaft, S. Kenig, and A. Moshonov. "The strain energy release rate of delamination in fabric-reinforced composites". Int. J. Adhesion and Adhesives, 8(2):85-91, 1988.

[48] R..H. Martin. Proc. American Soc. for Composites 3rd Tech. Conf., page 688, 1988.

[49] F.L. Matthews, P.F. Kilty, and E.W. Godwin. "A review of the strength of joints in fibre-reinforced plastics". Composites, 13(1):29-37, 1982.

[50] D.B. Miracle and S.L. Donaldson. Composites. In ASM Handbook, number 21. ASM International, 2001.

[51] N.K. Naik, K.S. Reddy, S. Meduri, N.B. Raju, P.D. Prasad, SK.N.M. Azad, P.A. Ogde, and B.C.K Reddy. "Interlaminar fracture characterization for plain weave fabric composites". Journal of Materials Science, 37:2983-2987, 2002.

[52] Newport Adhesives and Composites, INC. Newport 307 product data sheet. Technical report.

[53] D. J. Nicholls and J. P. Gallagher. "Determination of $G_{I c}$ in Angle Ply Composites Using a Cantilever Beam Teset Method". Journal of Reinforced Plastics and Composites, 2:2-17, 1983.

[54] T.K. O'Brian. "Characterization of delamination onset and growth in a composite laminate". Damage in composite materials. ASTM STP, 775(20):140-167, 1982.

[55] T.K. O'Brian and R.H. Martin. "Results of ASTM Round Robin Testing for Mode I Interlaminar Fracture Toughness of Composite Materials". Journal of Composites Technology and Research, 15(4):269, 1993.

[56] C.H. Popelar and M.F. Kanninen. "A Dynamic Viscoelastic Analysis of Crack Propagation and Crack Arrest in a Double Cantilever Beam Testing Specimen". In Crack Arrest Methodology and Applications, ASTM STP 711, G.T. Hahn and M.F. Kanninen, eds., pages 5-23. American Society for Testing and Materials, Philadelphia, 1980. 
[57] R.J. Roark. Formulas for Stress and Strain. MGraw-Hill Book Company, New York and London, second edition, eighth impression edition, 1943.

[58] P. Robinson and D.Q. Song. "A Modified DCB Specimen for Mode I Testing of Multidirectional Laminates". Journal of Composite Materials, pages 1554-1577, 1992.

[59] Ph. Rubbrecht and Verpoest I. The development of two new test methods to determine the mode I and mode II fracture toughness for varying fibre orientations at the interface. In $38^{\text {th }}$ International SAMPE Symposium, pages 875-877, Anaheim, CA, 1993.

[60] A. J. Russel and K. N. Street. "Factors Affecting the Interlaminar Fracture of Graphite/Epoxy Laminates". Progress in Science and Engineering of Composites, Proceedings, Fourth International Conference on Composite Materials(ICCMIV)):279-286, 1982.

[61] R.A. Schapery. "A Theory of Crack Initiation and Growth in Viscoelastic Media". International Journal of Fracture, 11:549-562, 1975.

[62] G.C. Sih and H. Liebowitz. "Mathematical Theories of Brittle Fracture". In H. Liebowitz, editor, Fracture-An Advanced Treatise, volume 2 of Academic, pages 67-190, New York, 1968.

[63] A.J. Smiley and R.B. Pipes. "Rate Effects on Mode I Interlaminar Fracture Toughness in Composite Materials". Journal of Composite Materials, 21:670-687, 1997.

[64] Strain Gage Installations with M-Bond 200 Adhesive. Instruction bulletin b-127-14. Technical Report 11127, Vishay Micro-Measurements, 2005.

[65] P. Suppakul and S. Bandyopadhyay. "The effect of weave pattern on the mode-I interlaminar fracture energy of E-glass/vinyl ester composites". Composites Science and Technology, 62:709-717, 2002.

[66] A. Szekrényes. "Overview on the experimental investigations of the fracture toughness in composite materials". Hungarian Electronic Journal of Sciences, 2002.

[67] H. Tada, P.C. Paris, and G.R. Irwin. The Stress Analysis of Cracks Handbook. Del Research Corporation, Dellertown, PA, third edition, 1973.

[68] J.M. Whitney. "Elasticity analysis of orhotropic beams under concentrated loads". Composites Science and Technology, 22:167-184, 1985.

[69] J.M. Whitney, C.E. Browning, and W. Hoogstedan. "A Double Cantilever Beam Test for Characterizing Mode I Delamination of Composite Materials". Journal of Reinforced Plastics and Composites, 1:297-330, 1982.

[70] J.G. Williams. "The Fracture Mechanics of Delamination Tests". Journal of Strain Analysis, 34(4):207-214, 1989. 
AppendixA

Four-Point Bend Schematics 


\section{A.1 Four-Point Base}

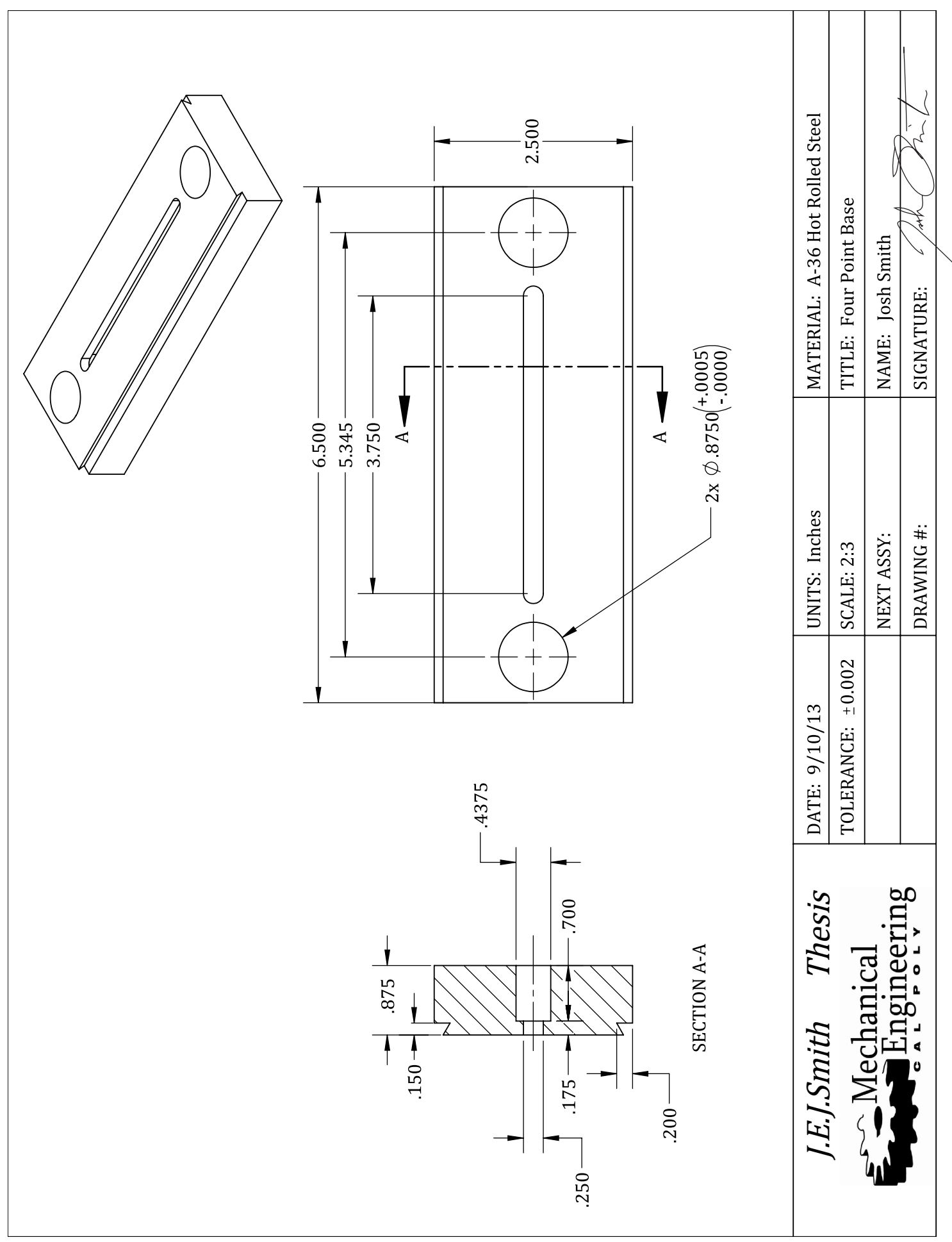




\section{A.2 Four-Point Standoff}

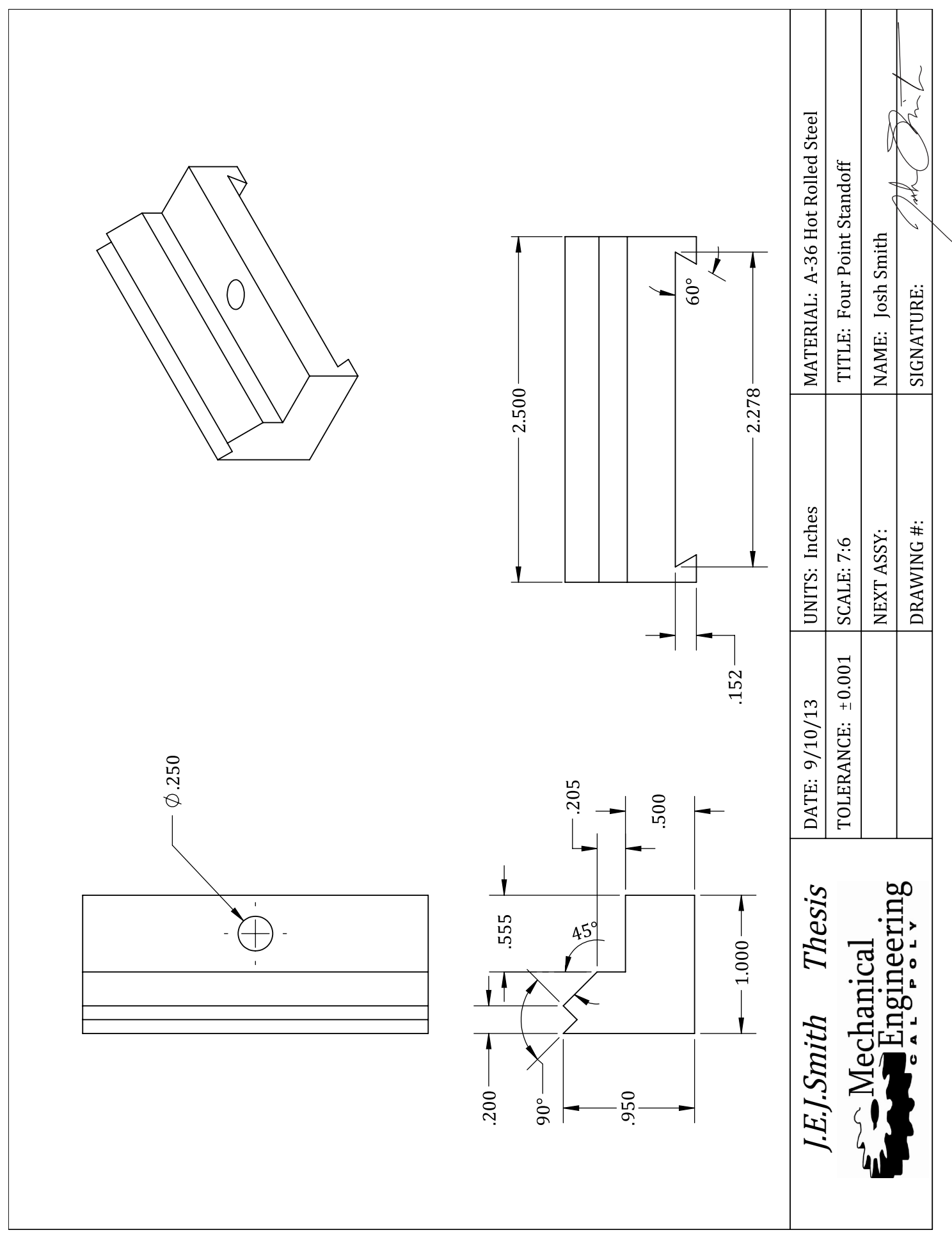




\section{AppendixB}

DCB Specimen Dimensions 
B.1 Tensile Specimen Dimensions

Table B.1: Tensile specimen average dimensions (inches)

\begin{tabular}{cccc}
\hline \hline Specimen Designation & $L$ & $b$ & $h$ \\
\hline $1-2$ & 9.75 & 0.396 & 0.0661 \\
$1-3$ & 9.75 & 0.341 & 0.0661 \\
$1-5$ & 9.75 & 0.396 & 0.0661 \\
$1-6$ & 9.75 & 0.410 & 0.0654 \\
\hline
\end{tabular}

B.2 In-Plane Shear Specimen Dimensions

Table B.2: In-plane shear specimen average dimensions (inches)

\begin{tabular}{cccc}
\hline \hline Specimen Designation & $L$ & $b$ & $h$ \\
\hline $3-1$ & 9.75 & 1.015 & 0.1366 \\
$3-2$ & 9.75 & 0.965 & 0.1385 \\
$3-3$ & 9.75 & 0.987 & 0.1386 \\
$3-4$ & 9.75 & 1.015 & 0.1357 \\
$3-5$ & 9.75 & 0.974 & 0.1347 \\
$3-6$ & 9.75 & 1.002 & 0.1393 \\
$3-7$ & 9.75 & 1.010 & 0.1312 \\
\hline
\end{tabular}

B.3 Short Beam Shear Specimen Dimensions

Table B.3: Short beam shear specimen average dimensions (inches)

\begin{tabular}{cccc}
\hline \hline Specimen Designation & $L$ & $b$ & $h$ \\
\hline $12-5$ & 1.014 & 0.335 & 0.1695 \\
$12-6$ & 1.018 & 0.335 & 0.1690 \\
$12-7$ & 1.018 & 0.334 & 0.1690 \\
$12-8$ & 1.018 & 0.334 & 0.1682 \\
$12-9$ & 1.012 & 0.326 & 0.1694 \\
$12-10$ & 1.017 & 0.326 & 0.1681 \\
$13-5$ & 1.018 & 0.330 & 0.1705 \\
$13-6$ & 1.011 & 0.331 & 0.1700 \\
$13-7$ & 1.010 & 0.333 & 0.1716 \\
$13-8$ & 1.005 & 0.333 & 0.1720 \\
$13-9$ & 1.038 & 0.332 & 0.1686 \\
$13-10$ & 1.011 & 0.334 & 0.1708 \\
\hline
\end{tabular}




\section{B.4 Flex Specimen Dimensions}

Table B.4: Flex specimen average dimensions (inches)

\begin{tabular}{cccc}
\hline \hline Specimen Designation & $L$ & $b$ & $h$ \\
\hline $12-1$ & 3.280 & 0.529 & 0.1687 \\
$12-2$ & 3.280 & 0.549 & 0.1668 \\
$12-3$ & 3.280 & 0.559 & 0.1696 \\
$12-4$ & 3.278 & 0.541 & 0.1700 \\
$12-11$ & 3.273 & 0.532 & 0.1679 \\
$12-12$ & 3.277 & 0.538 & 0.1691 \\
$13-1$ & 3.264 & 0.548 & 0.1704 \\
$13-2$ & 3.258 & 0.555 & 0.1702 \\
$13-3$ & 3.269 & 0.546 & 0.1719 \\
$13-4$ & 3.272 & 0.579 & 0.1725 \\
$13-11$ & 3.251 & 0.539 & 0.1713 \\
$13-12$ & 3.255 & 0.530 & 0.1709 \\
\hline
\end{tabular}

B.5 DCB Specimen Dimensions

Table B.5: DCB specimen average dimensions (inches)

\begin{tabular}{cccccccccc}
\hline \hline Specimen & $a_{o}$ & $L$ & $b$ & $h$ & Specimen & $a_{o}$ & $L$ & $b$ & $h$ \\
\hline $6-1$ & 1.949 & 4.506 & 0.993 & 0.1164 & $9-1$ & 1.969 & 6.875 & 1.009 & 0.1375 \\
$6-2$ & 1.949 & 4.497 & 0.999 & 0.1208 & $9-2$ & 2.087 & 6.875 & 1.022 & 0.1380 \\
$6-3$ & 1.929 & 4.510 & 1.106 & 0.1209 & $9-3$ & 2.008 & 6.875 & 1.012 & 0.1384 \\
$6-4$ & 1.890 & 4.512 & 1.004 & 0.1239 & $9-4$ & 2.087 & 6.875 & 1.024 & 0.1381 \\
$6-5$ & 1.909 & 4.505 & 0.980 & 0.1233 & $9-5$ & 2.067 & 6.875 & 1.016 & 0.1380 \\
$6-6$ & 1.909 & 4.495 & 0.952 & 0.1173 & $10-1$ & 2.106 & 6.810 & 1.048 & 0.1312 \\
$7-1$ & 2.165 & 6.500 & 0.960 & 0.1303 & $10-2$ & 2.087 & 6.810 & 1.025 & 0.1322 \\
$7-2$ & 2.165 & 6.500 & 0.996 & 0.1441 & $10-3$ & 2.087 & 6.810 & 1.031 & 0.1335 \\
$7-3$ & 2.205 & 6.500 & 0.987 & 0.1483 & $10-4$ & 2.067 & 6.810 & 1.030 & 0.1336 \\
$7-4$ & 2.165 & 6.500 & 0.963 & 0.1450 & $10-5$ & 2.087 & 6.810 & 1.024 & 0.1331 \\
$7-5$ & 2.165 & 6.500 & 1.012 & 0.1336 & $10-6$ & 2.106 & 6.810 & 1.039 & 0.1324 \\
$8-1$ & 2.126 & 6.840 & 0.966 & 0.1242 & $11-1$ & 2.047 & 6.780 & 1.008 & 0.1391 \\
$8-2$ & 2.087 & 6.840 & 1.014 & 0.1294 & $11-2$ & 2.047 & 6.780 & 1.040 & 0.1400 \\
$8-3$ & 2.087 & 6.830 & 0.975 & 0.1331 & $11-3$ & 2.047 & 6.780 & 1.036 & 0.1382 \\
$8-4$ & 2.106 & 6.840 & 1.003 & 0.1336 & $11-4$ & 2.047 & 6.780 & 1.012 & 0.1382 \\
$8-5$ & 2.126 & 6.840 & 1.009 & 0.1310 & $11-5$ & 2.047 & 6.780 & 1.011 & 0.1388 \\
$8-6$ & 2.126 & 6.810 & 1.012 & 0.1272 & $11-6$ & 2.047 & 6.750 & 1.016 & 0.1385 \\
\hline
\end{tabular}




\section{AppendixC}

\section{Hinge Schematics}


C.1 Upper-Jaw Hinge

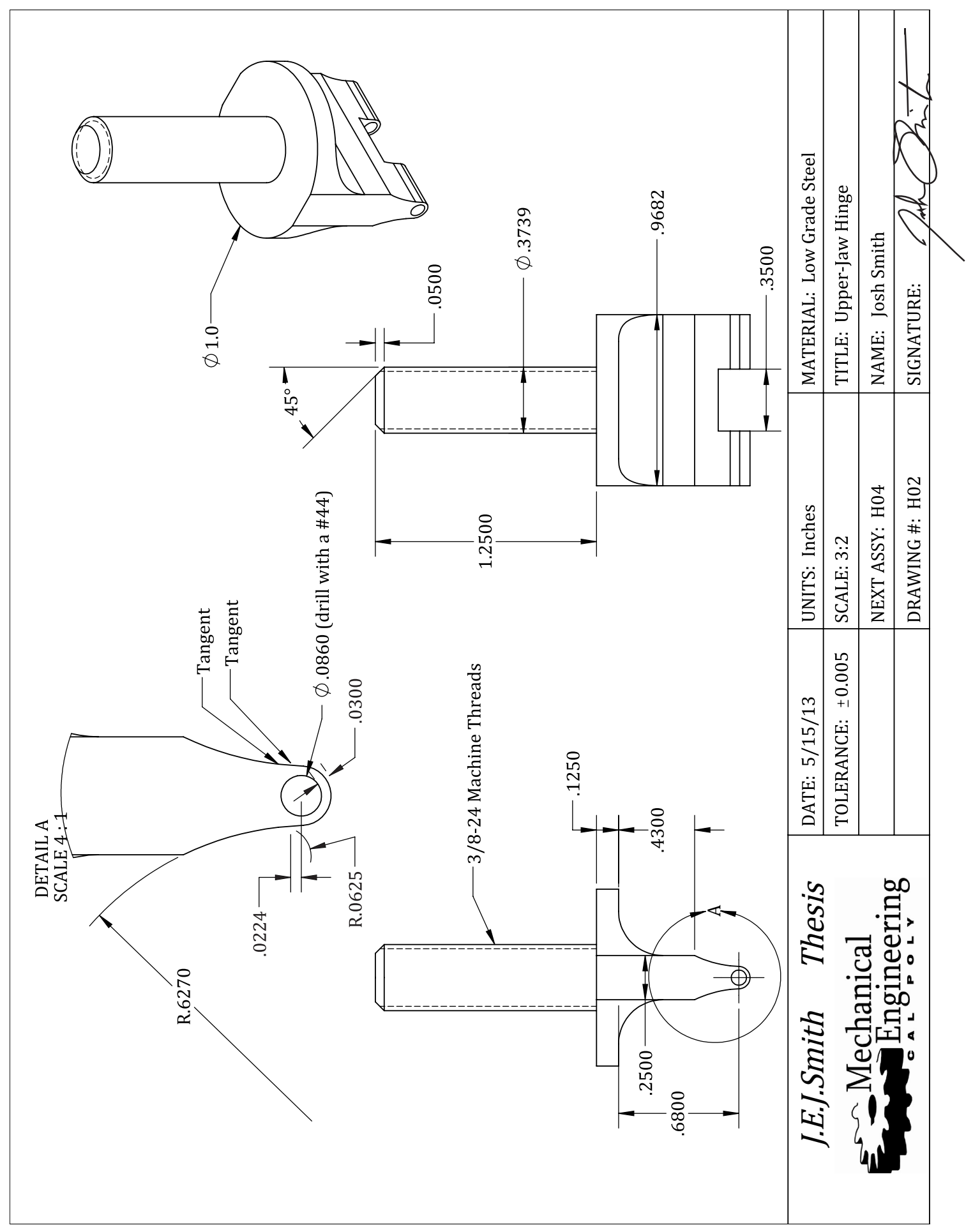


C.2 Bondable Hinges

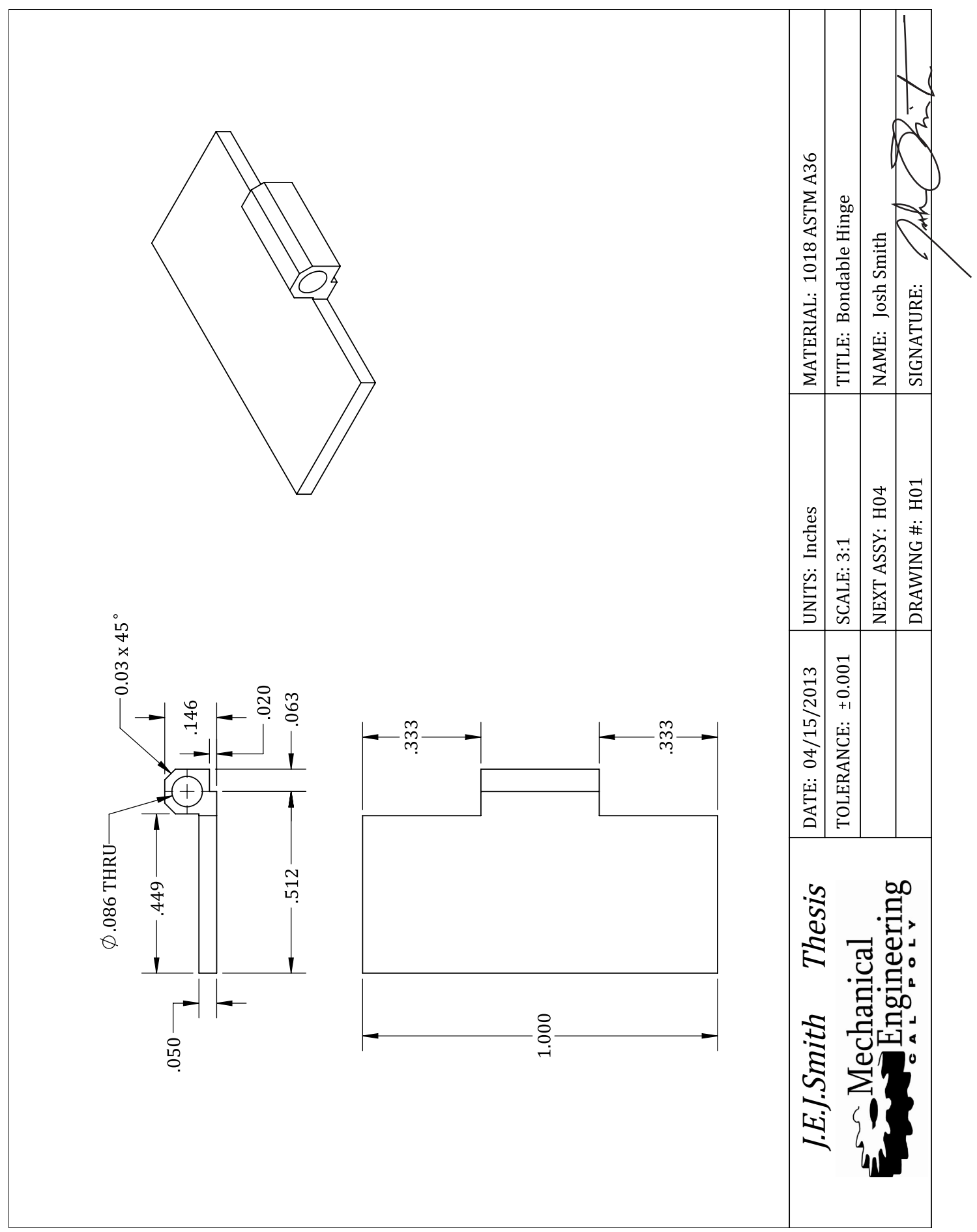




\section{C.3 Lower-Jaw Hinge}

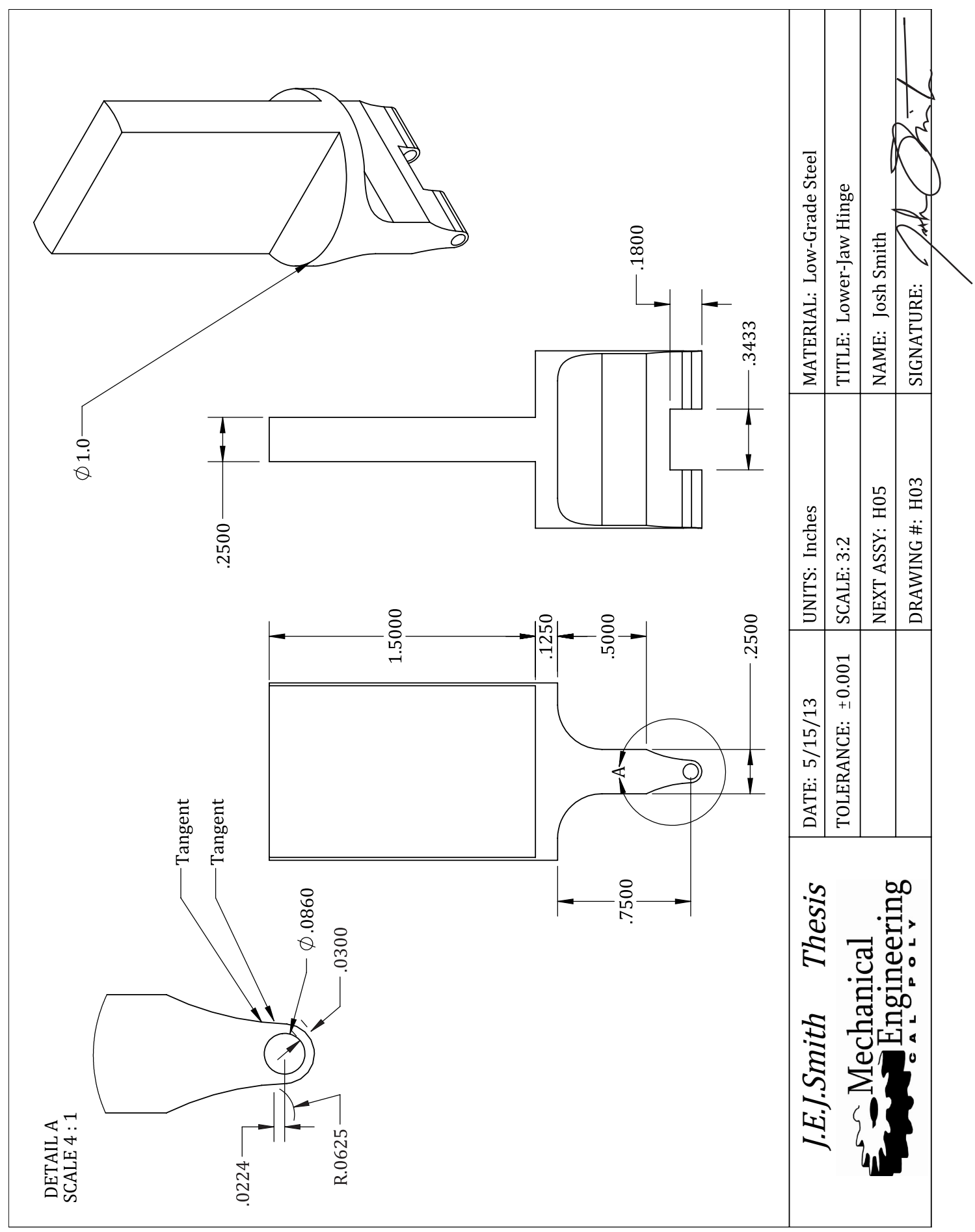




\section{C.4 Pins}

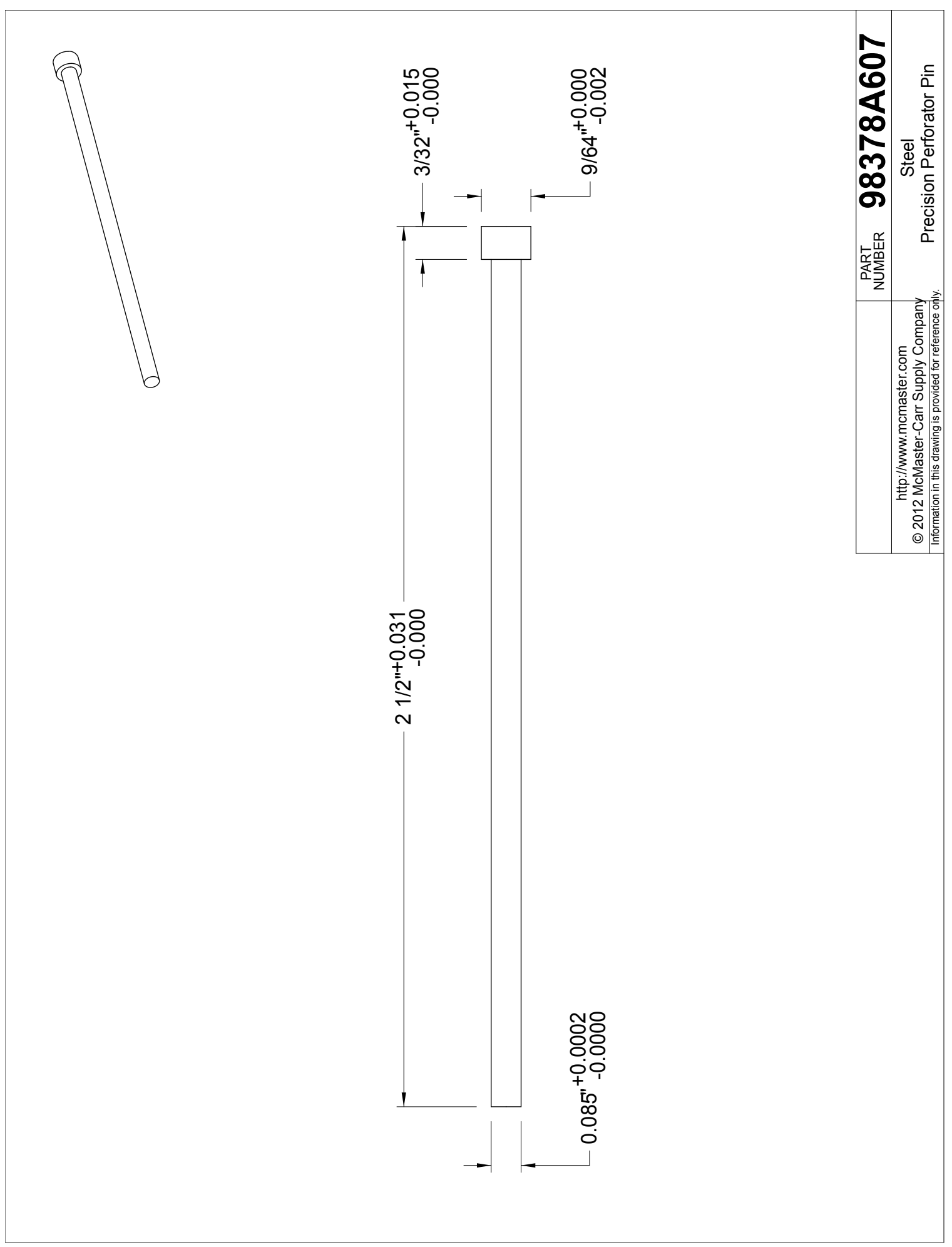


AppendixD

Microscope Mount 
D.1 Microscope Mount Details

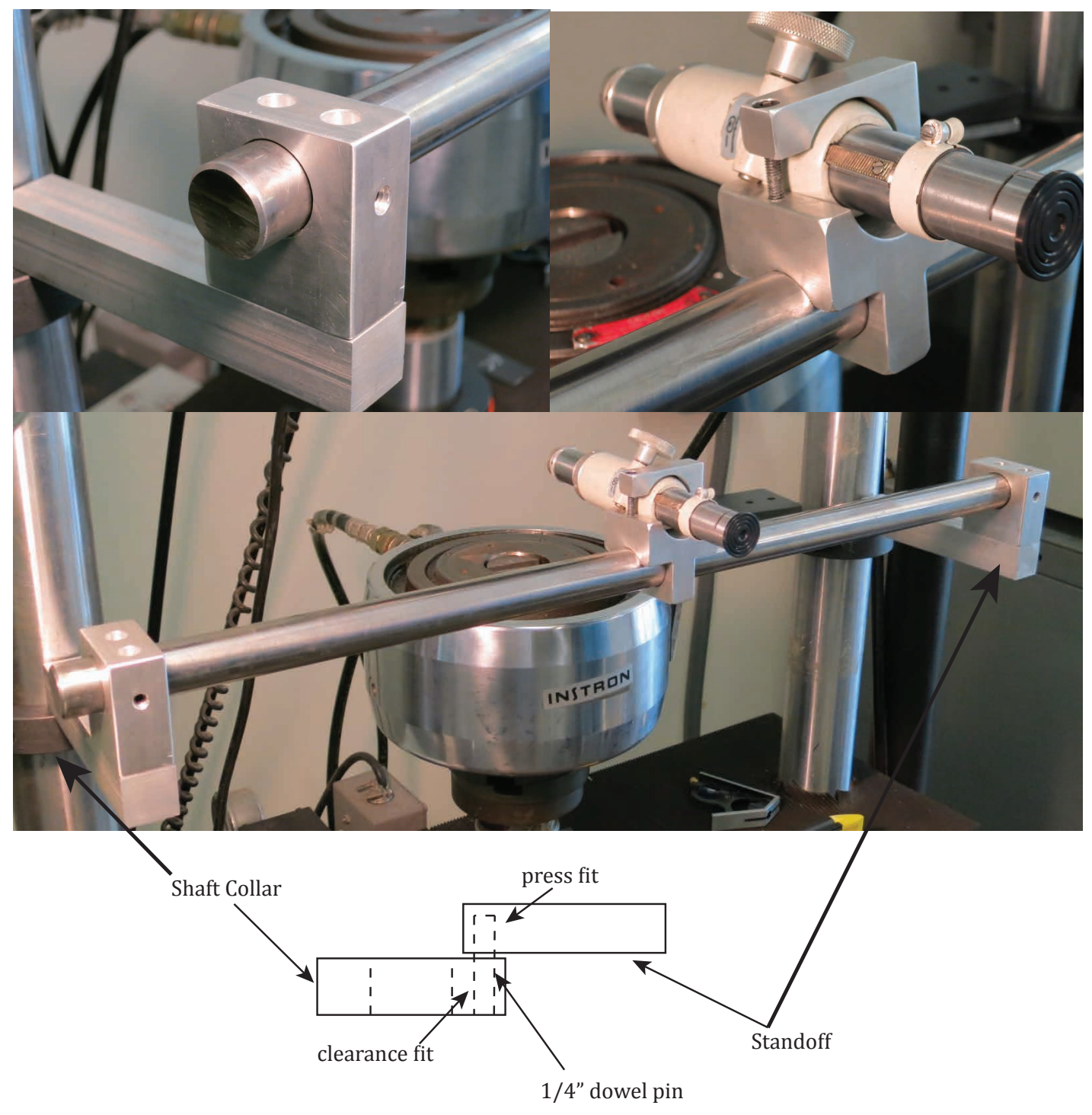


AppendixE

Load Cell Calibration 


\section{E.1 Calibration Procedure for LCH-100 Load Cell}

1. Plug in the Omega ${ }^{\circledR}$ DP80 series digital strain indicator and attach the load cell to the external port

2. Press the PGM button until "Program" is displayed

3. Press the $\Downarrow$ button until "Setup" is displayed and press ENT

4. Press the $\Downarrow$ button until "3PT CAL" is displayed and press ENT

5. Wait until "SET PNT 1" is displayed. Add the smallest of 3 masses (The Upper-Jaw Hinge with the pin inserted, see Figure E.1)

6. Press ENT. A "+" will be displayed. If the load is to be a positive value, press ENT. If it is to be negative, press $\Downarrow$ until "-" is displayed and press ENT

7. Use the $\Uparrow$ and $\Downarrow$ buttons to change the force applied. Use the ENT button to accept each significant digit. When ENT is pressed nine times, the load set is complete for the first calibration load

8. "MID PNT?" will be displayed. Accept the default "YES" option by pressing ENT

9. "SET MID" will be displayed. Add the second calibration mass and press ENT (A 3/8" bolt, a 10lb weight, and the Upper-Jaw Hinge and pin, see Figure E.2)

10. Repeat steps 6 and 7

11. "SET PNT 2" will be displayed. Add the third calibration mass and press ENT (Another 10lb weight, see Figure E.3)

12. $\quad$ Repeat steps 6 and 7

13. "SETUP OK" will be displayed. Press the PGM button to complete the calibration

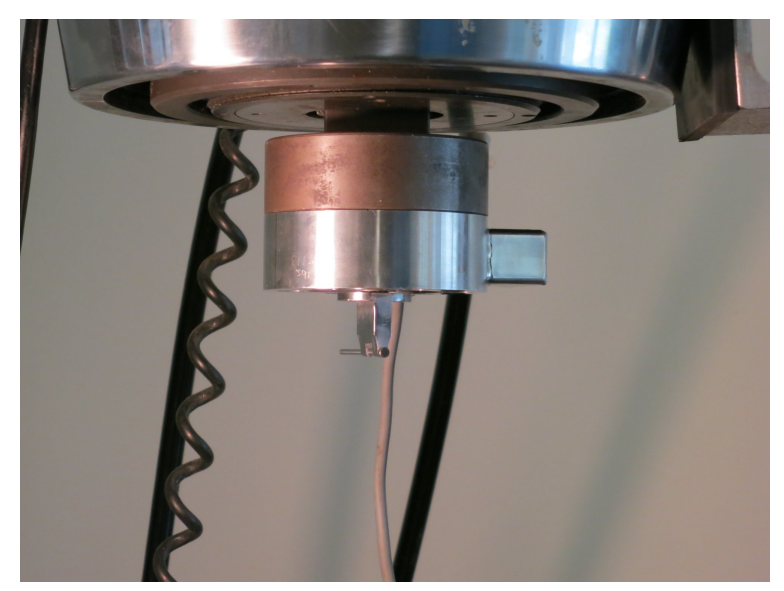

Figure E.1: First calibration weight 


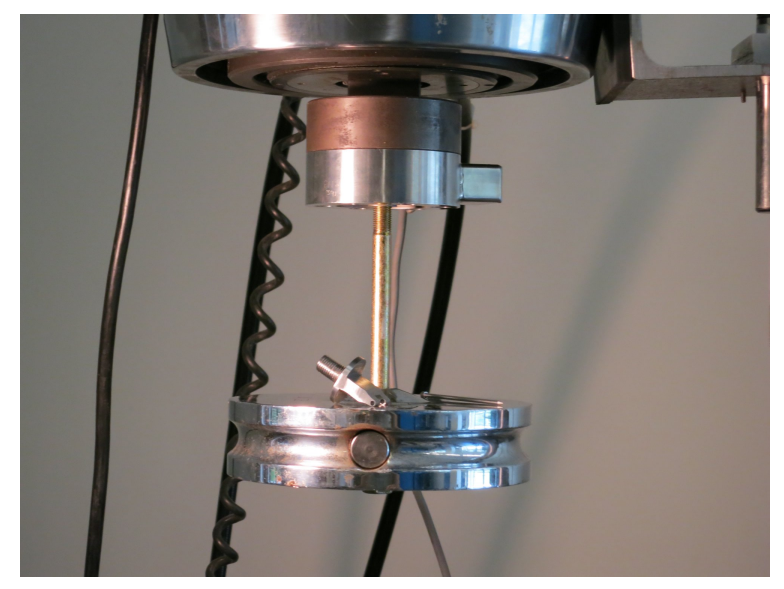

Figure E.2: Second calibration weight

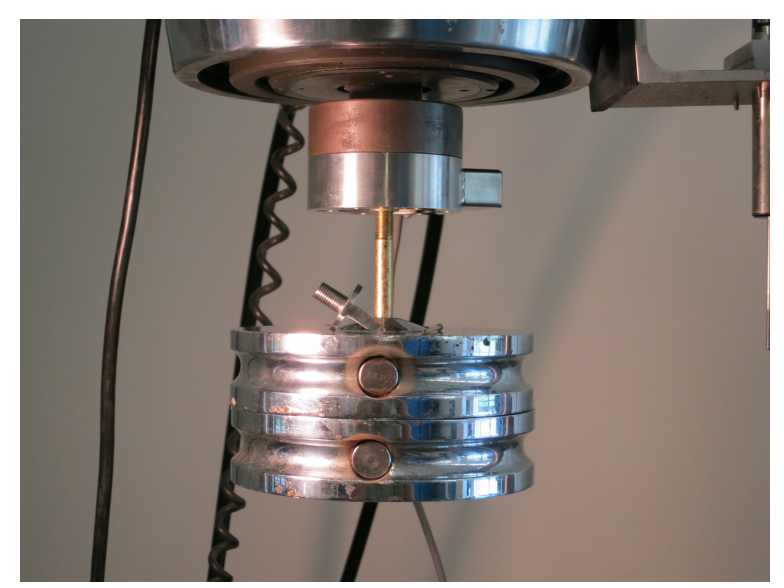

Figure E.3: Third calibration weight 


\begin{abstract}
AppendixF
DCB Specimen Load-Displacement Plots
\end{abstract}


F.1 $P-\delta$ Plots for Plate 6
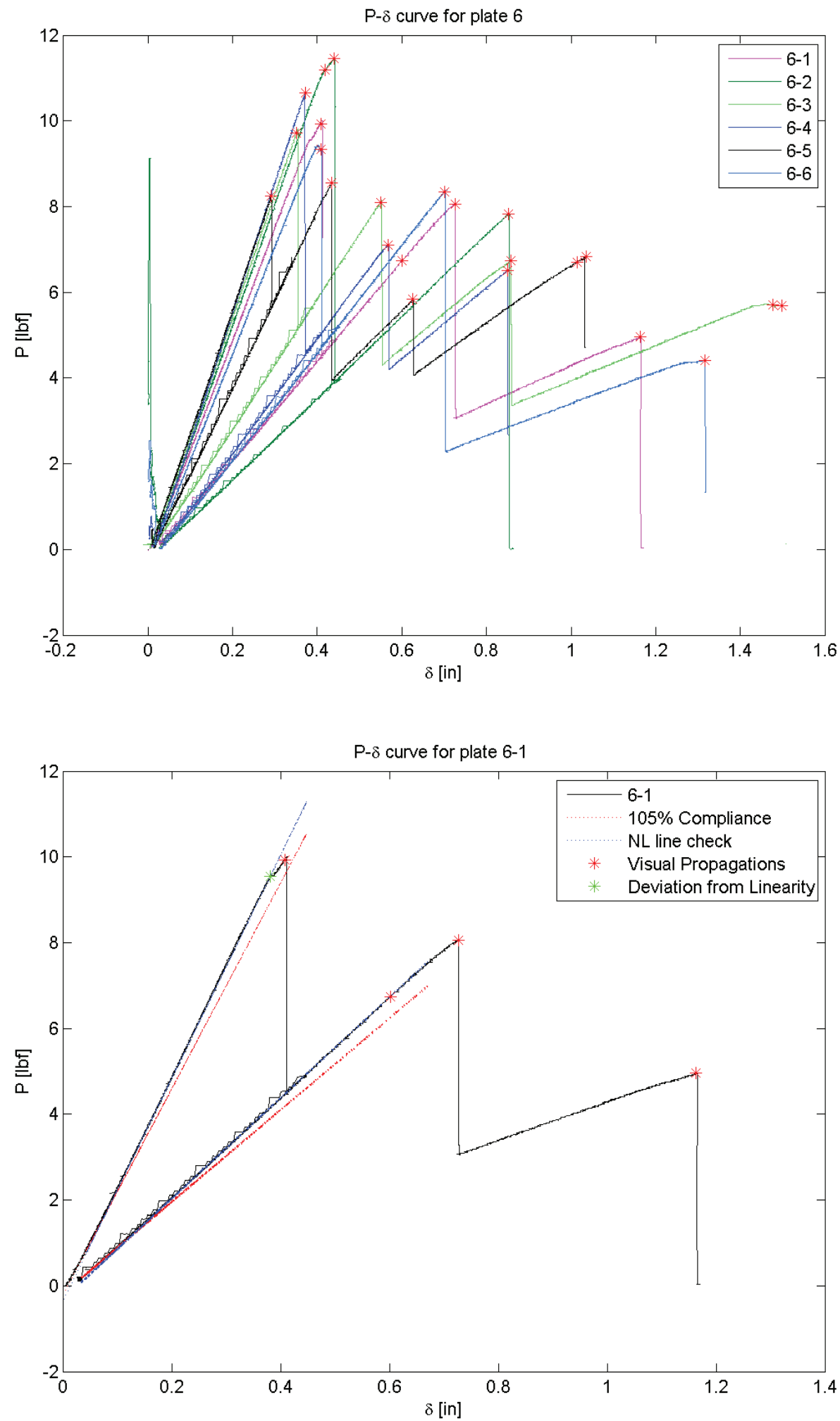

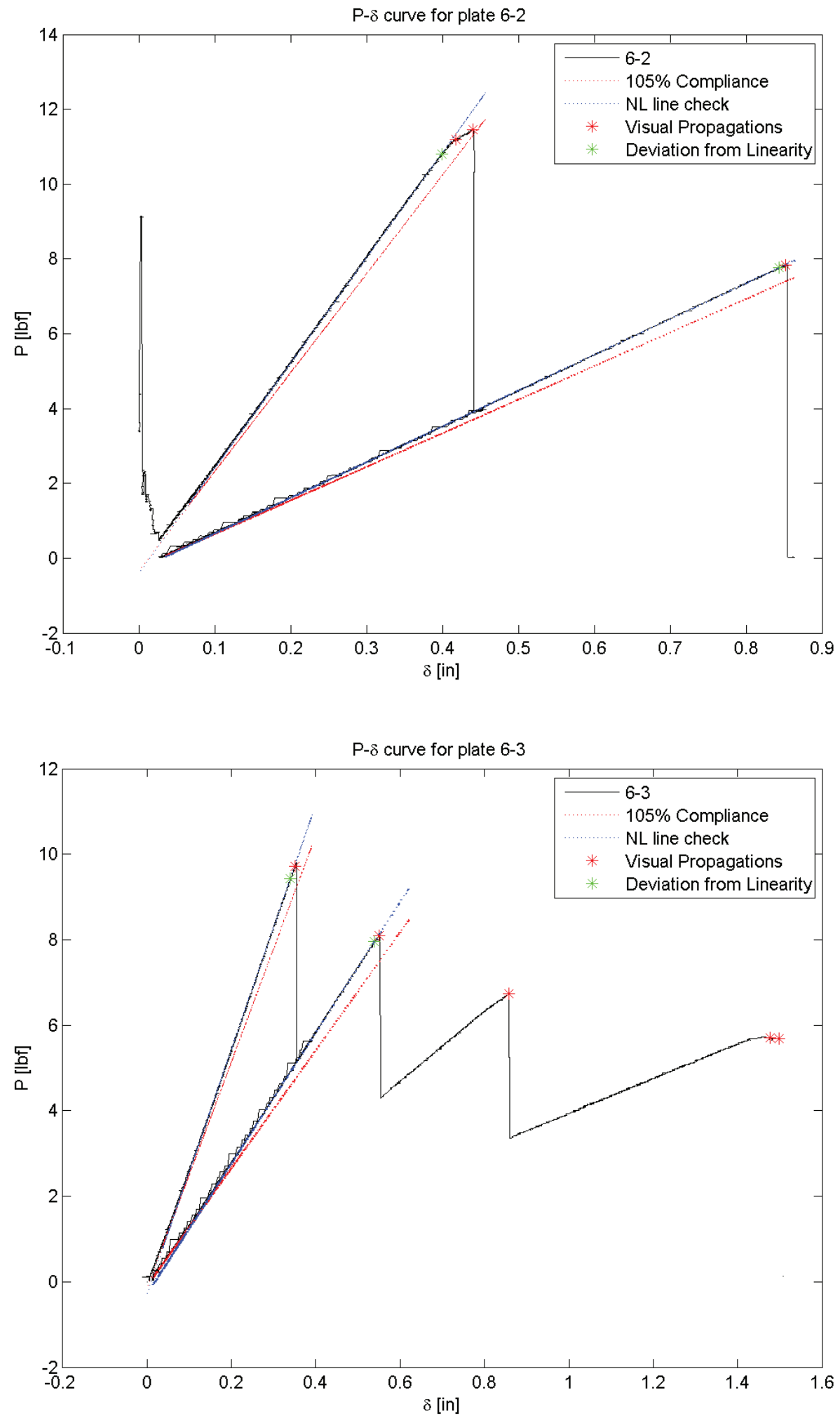

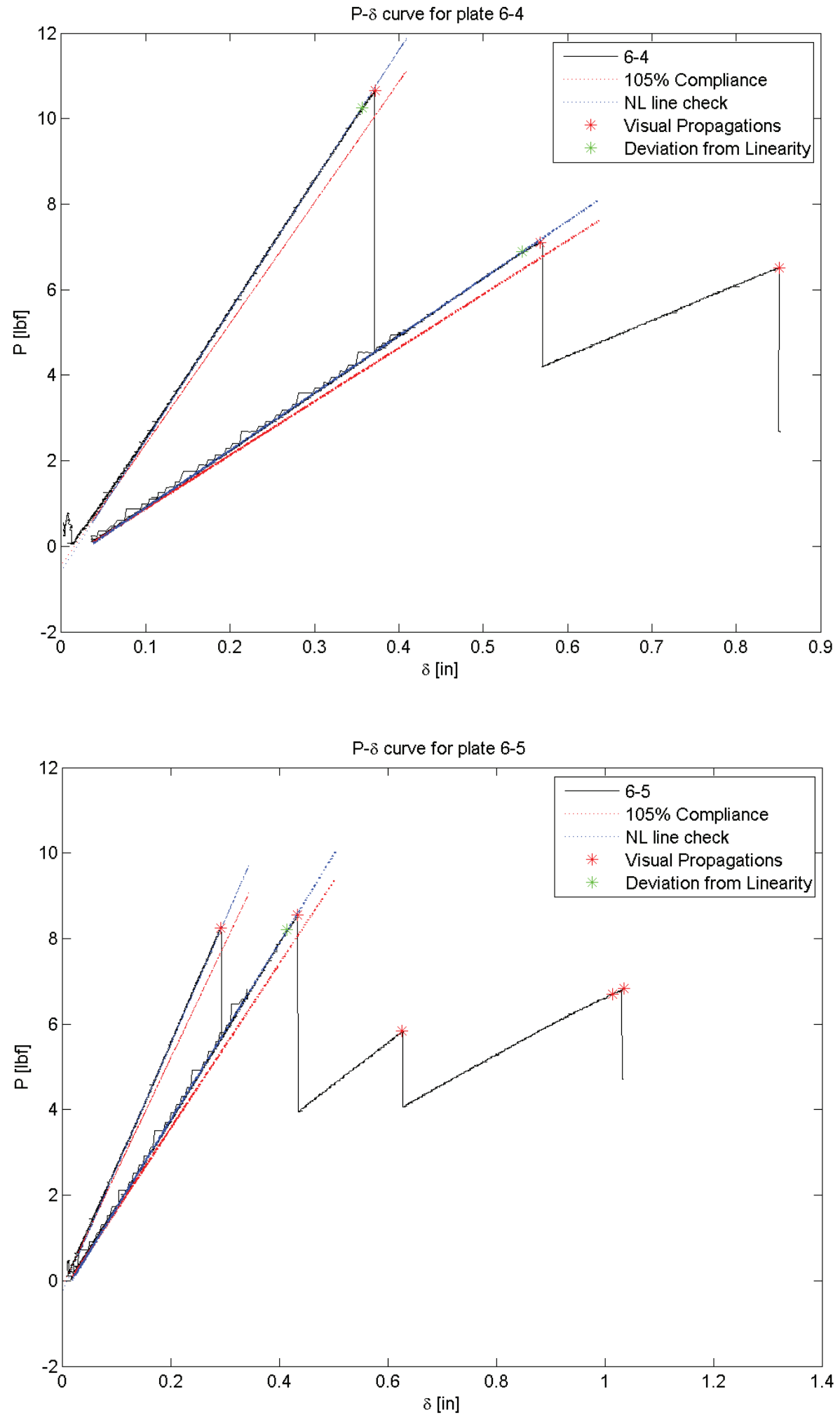


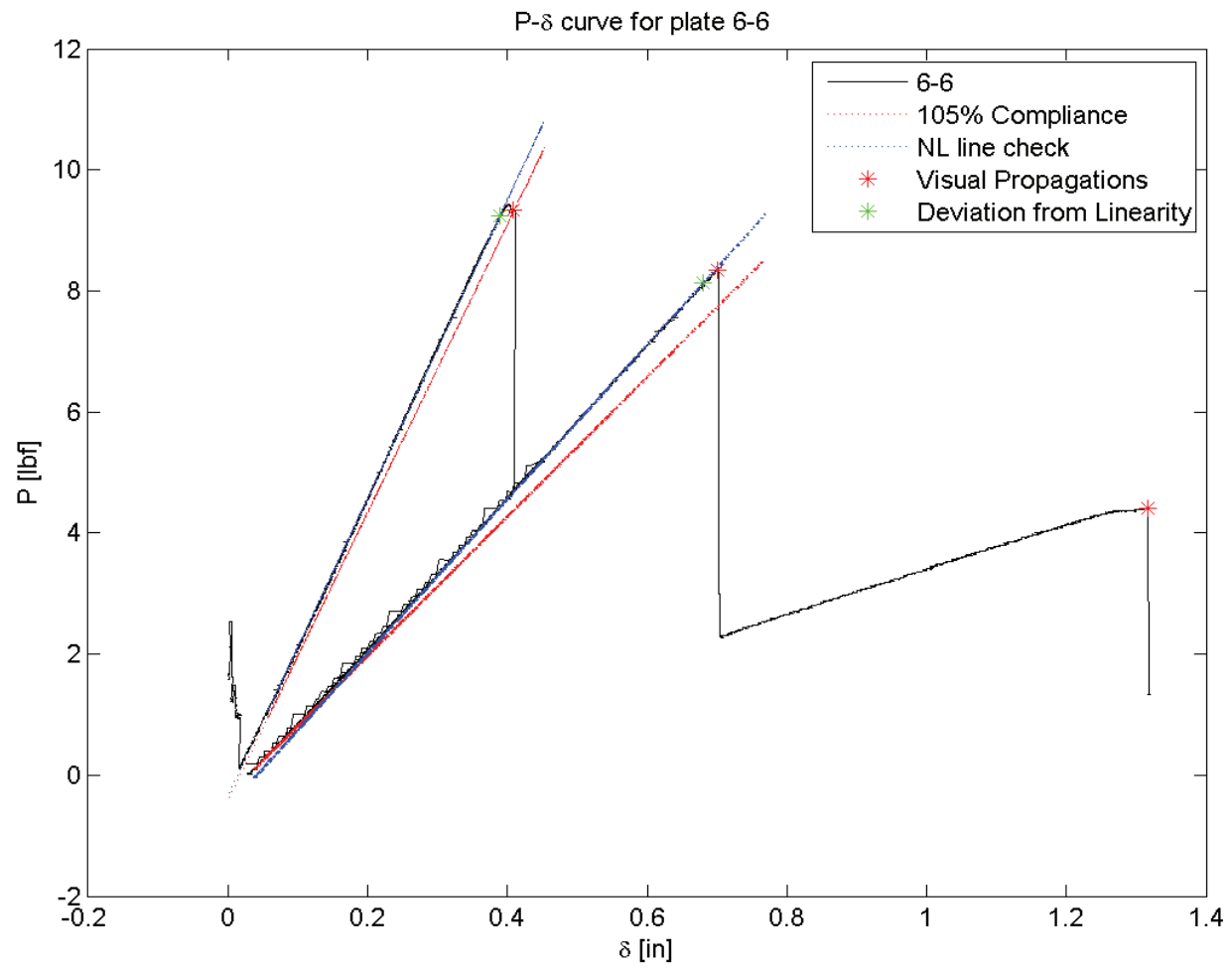


F.2 $P-\delta$ Plots for Plate 7
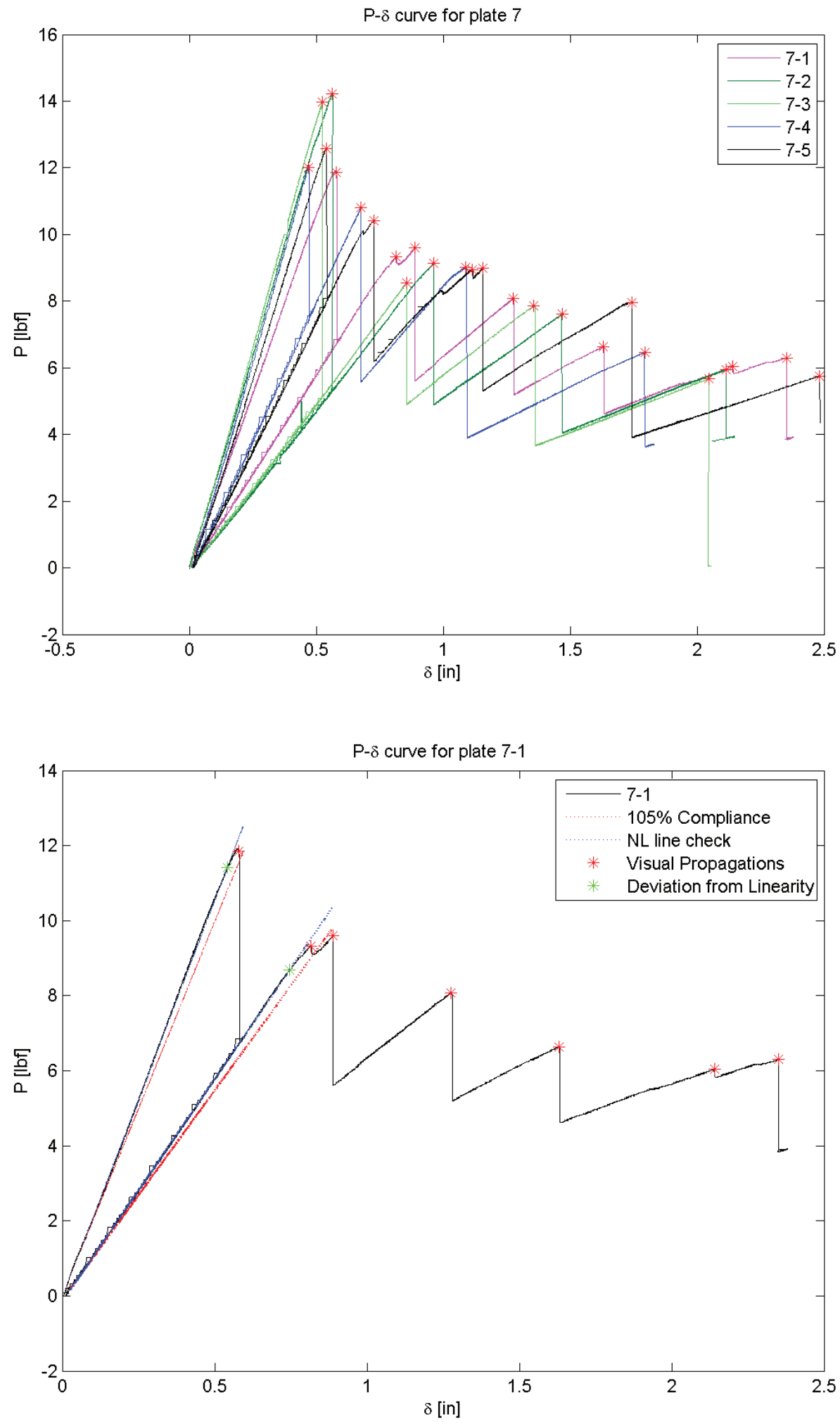

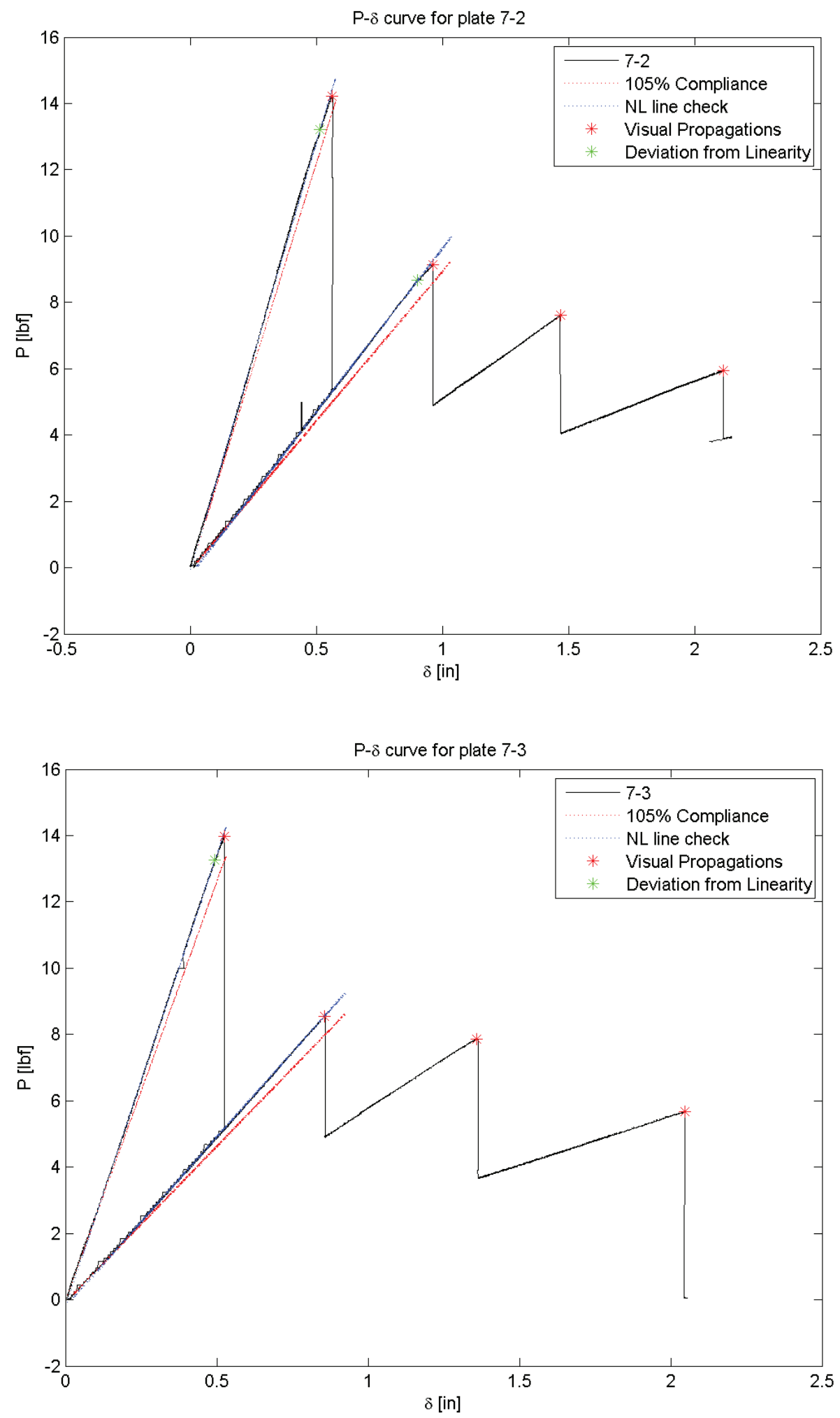

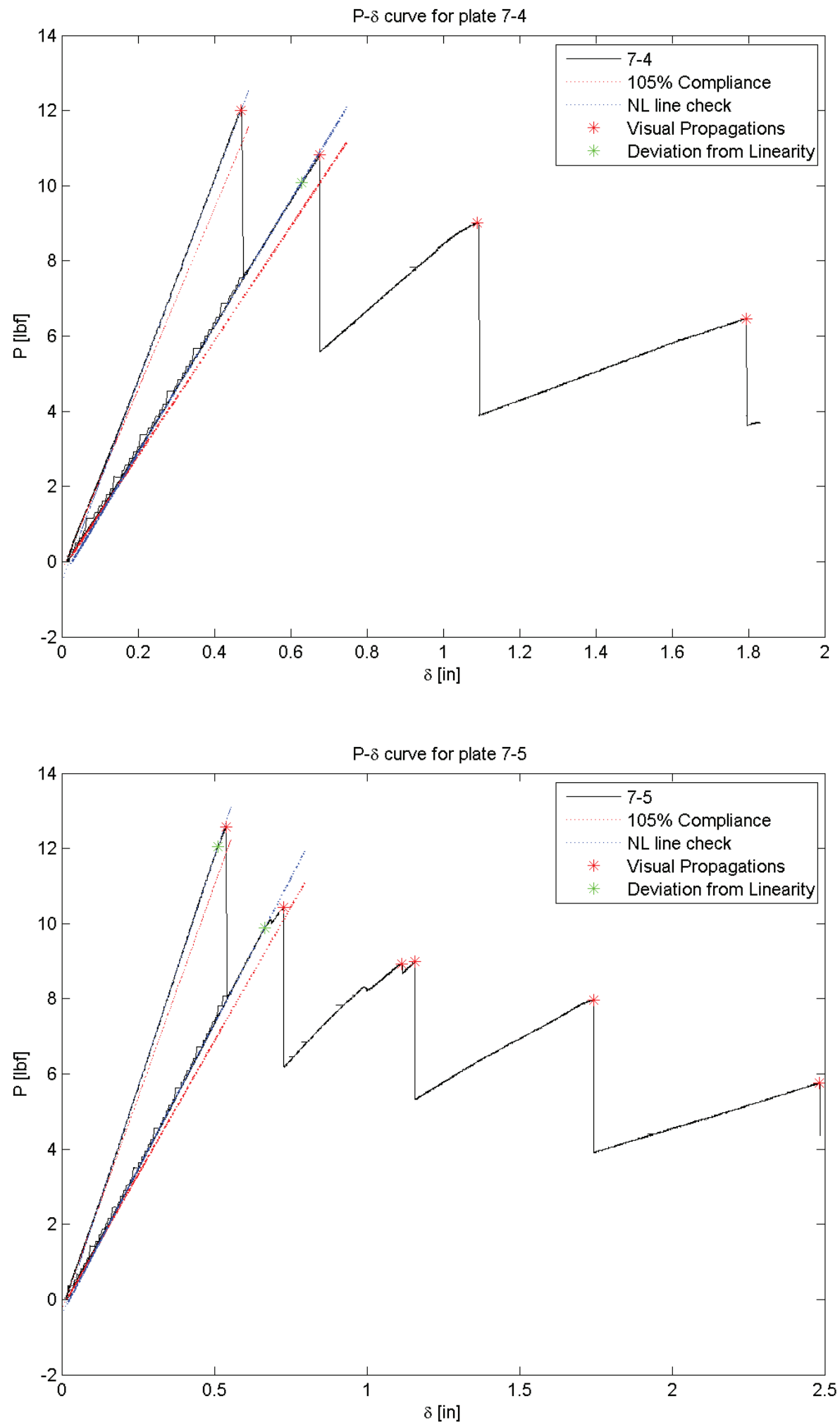
F.3 $P-\delta$ Plots for Plate 8
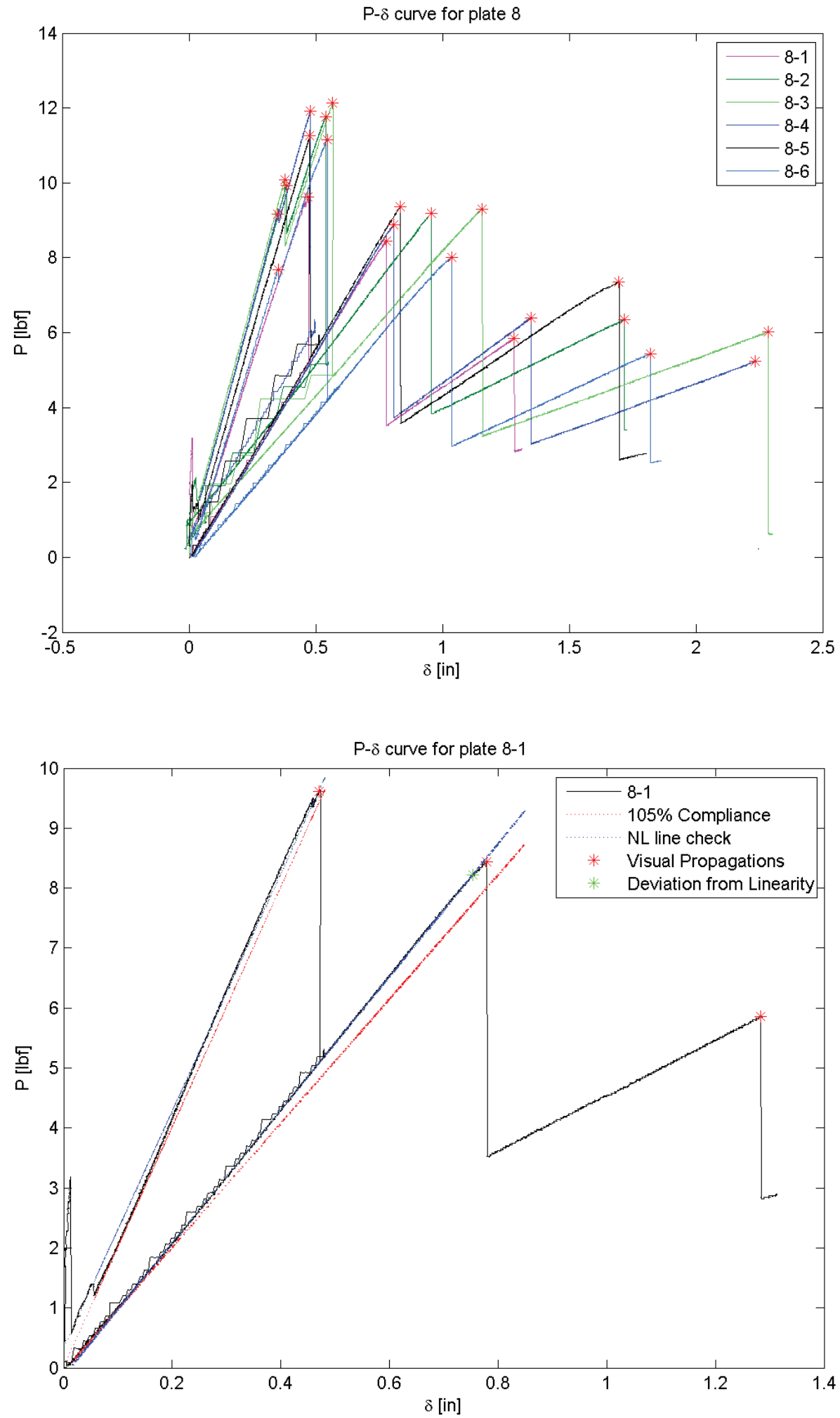

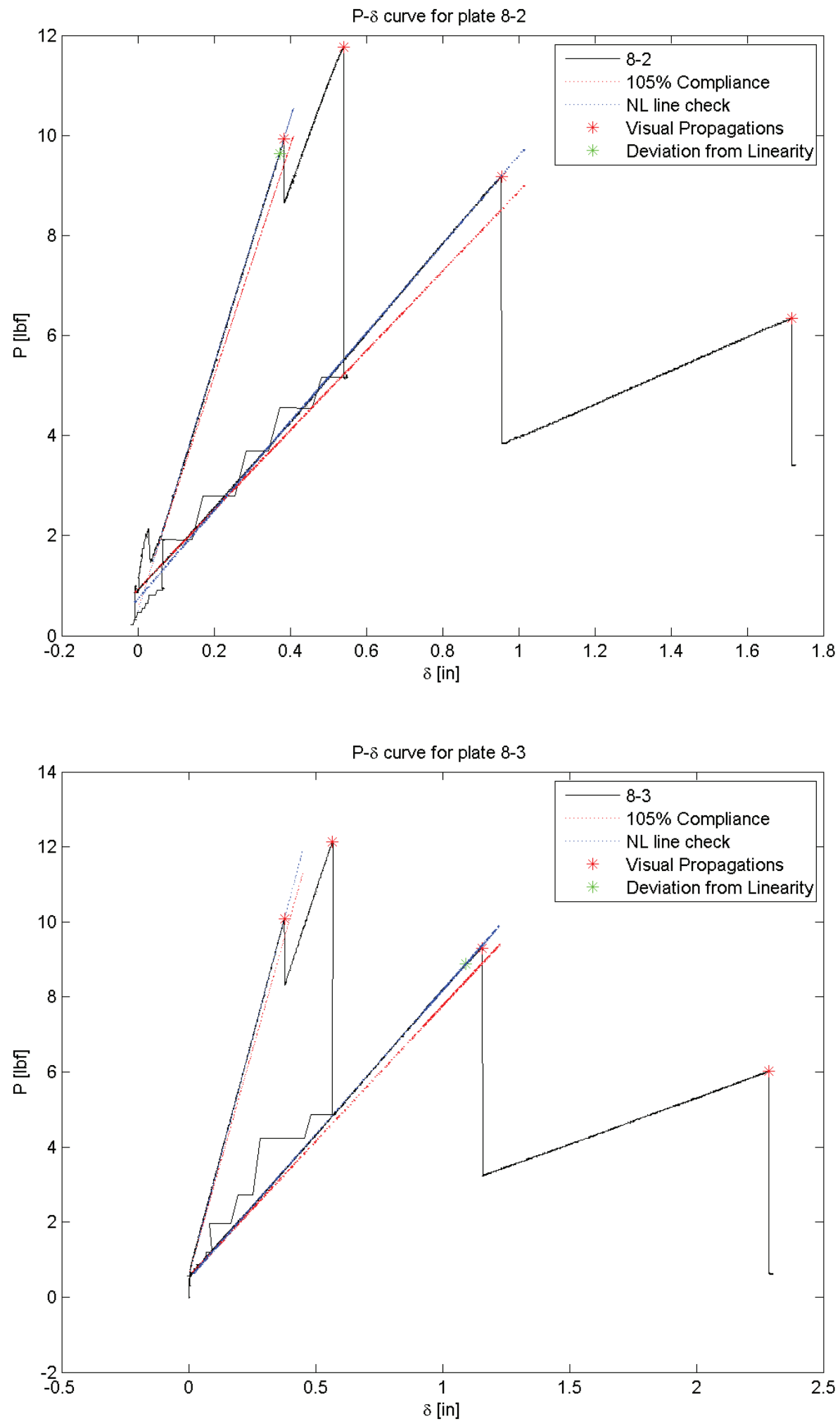

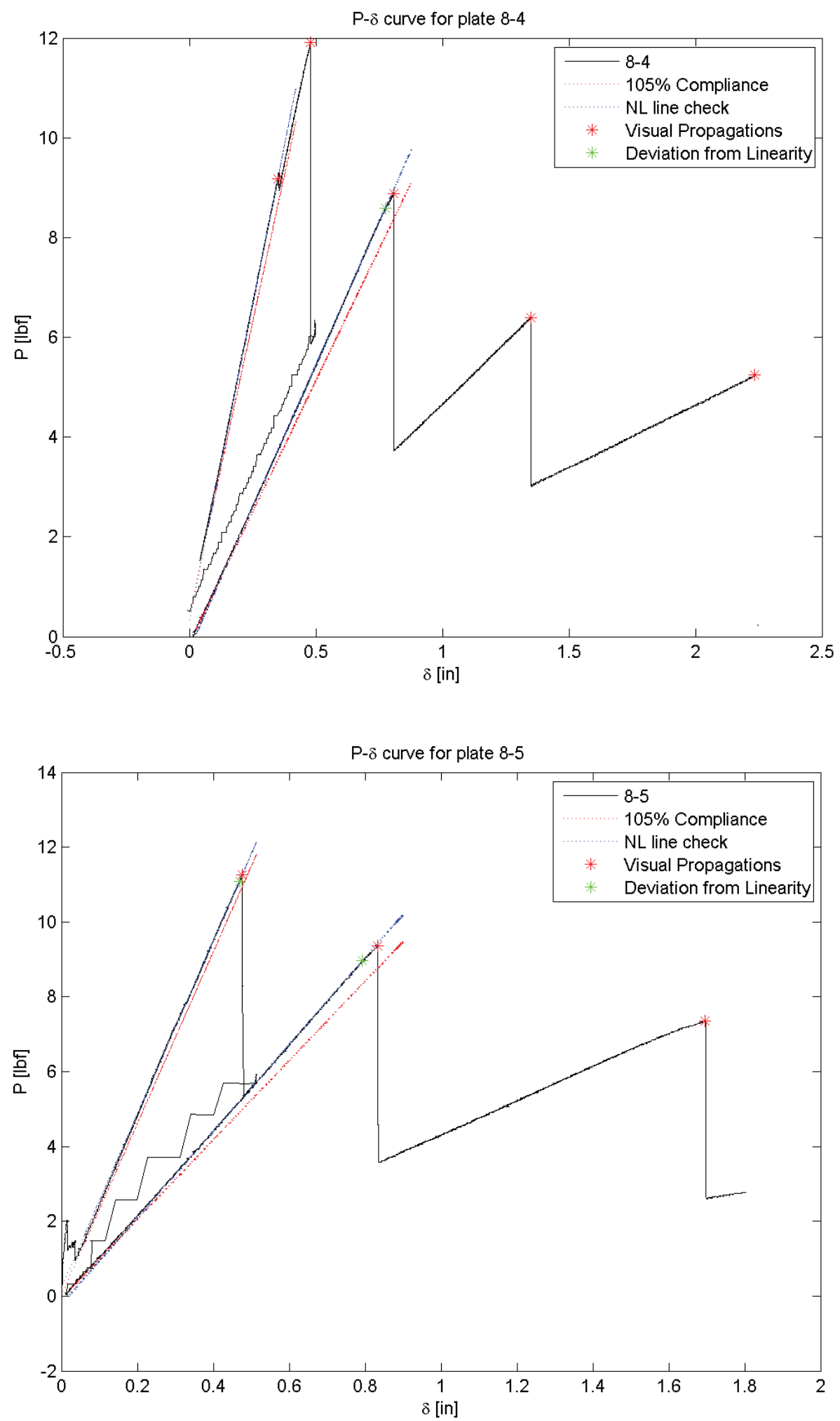


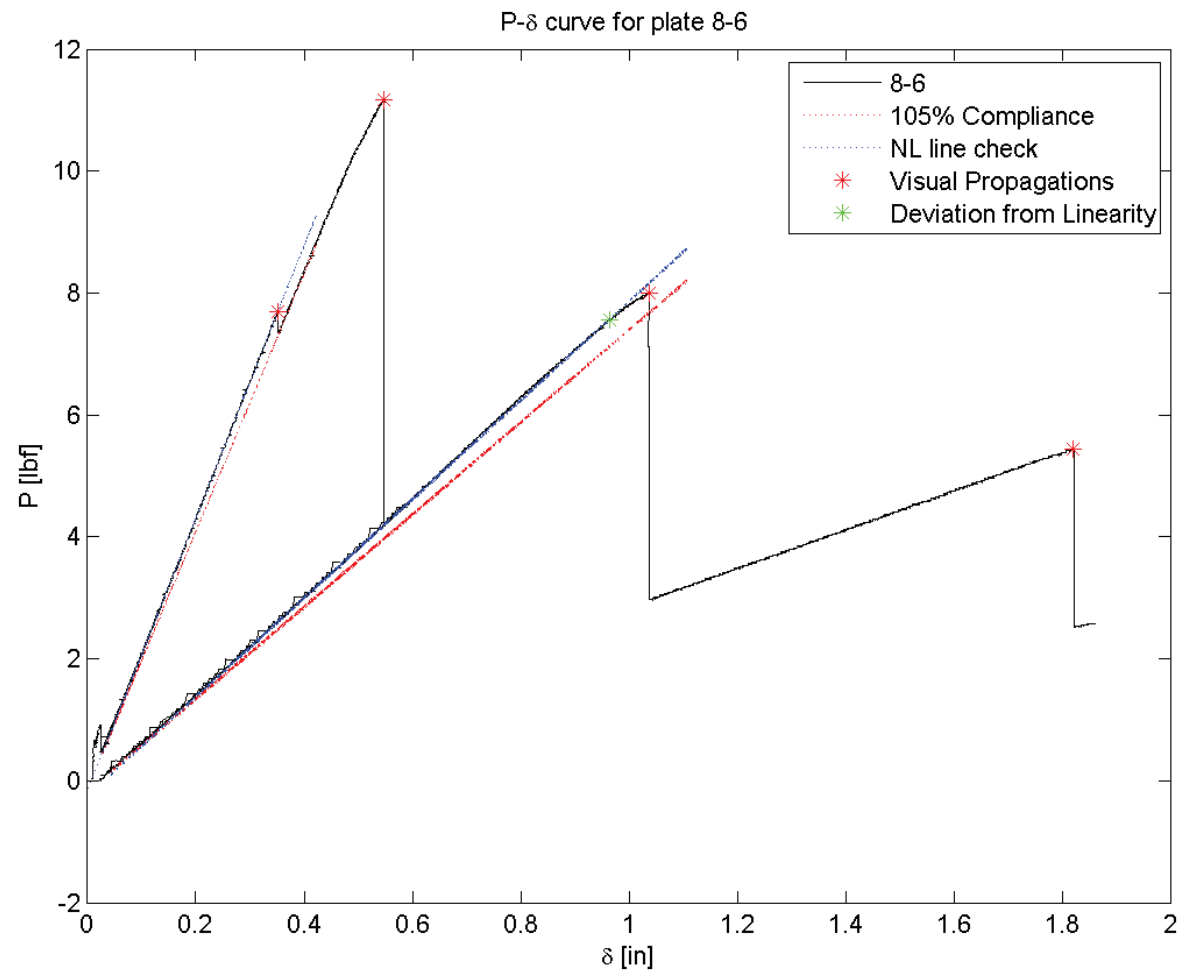


F.4 $P-\delta$ Plots for Plate 9
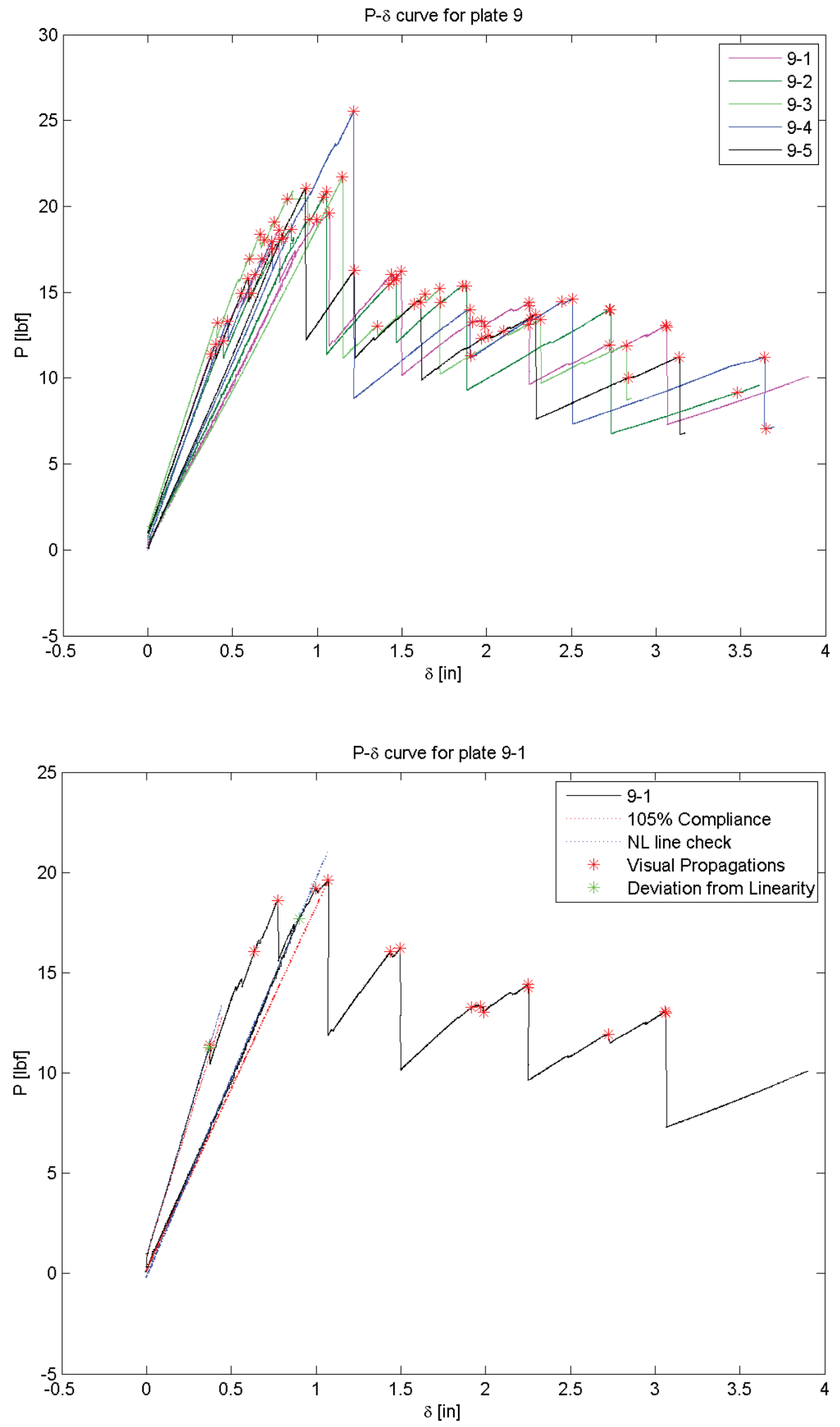

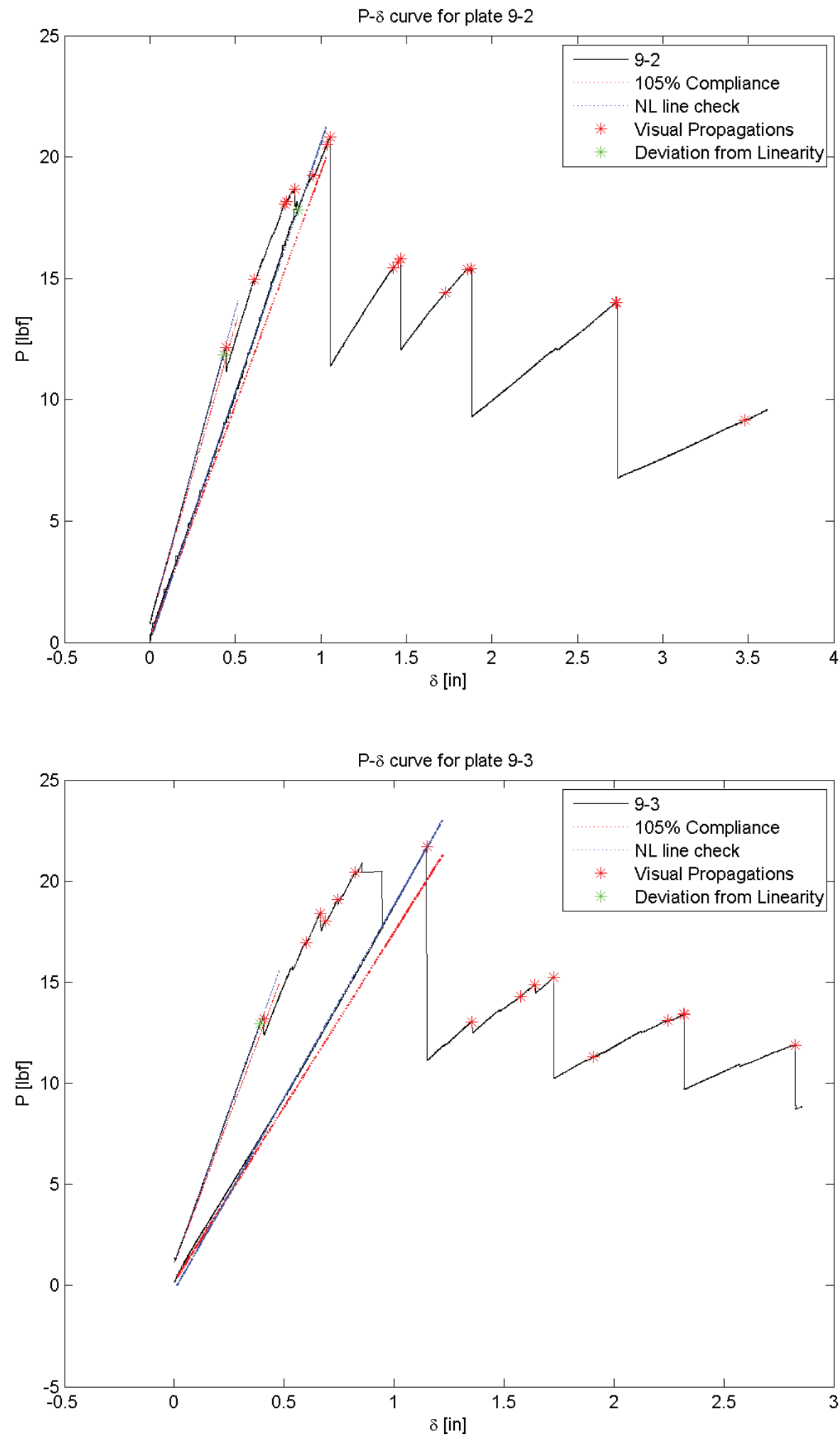

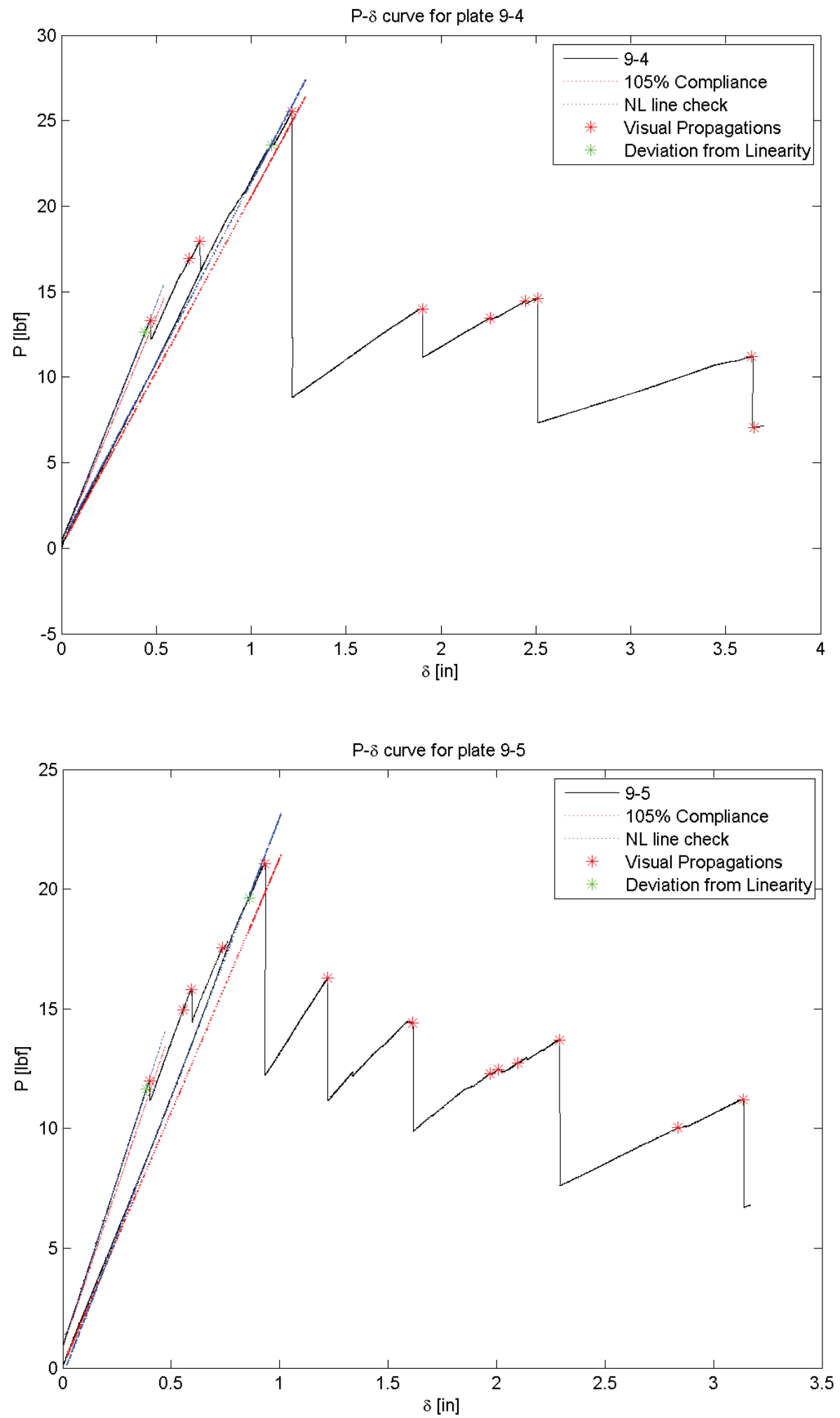
F.5 $P-\delta$ Plots for Plate 10
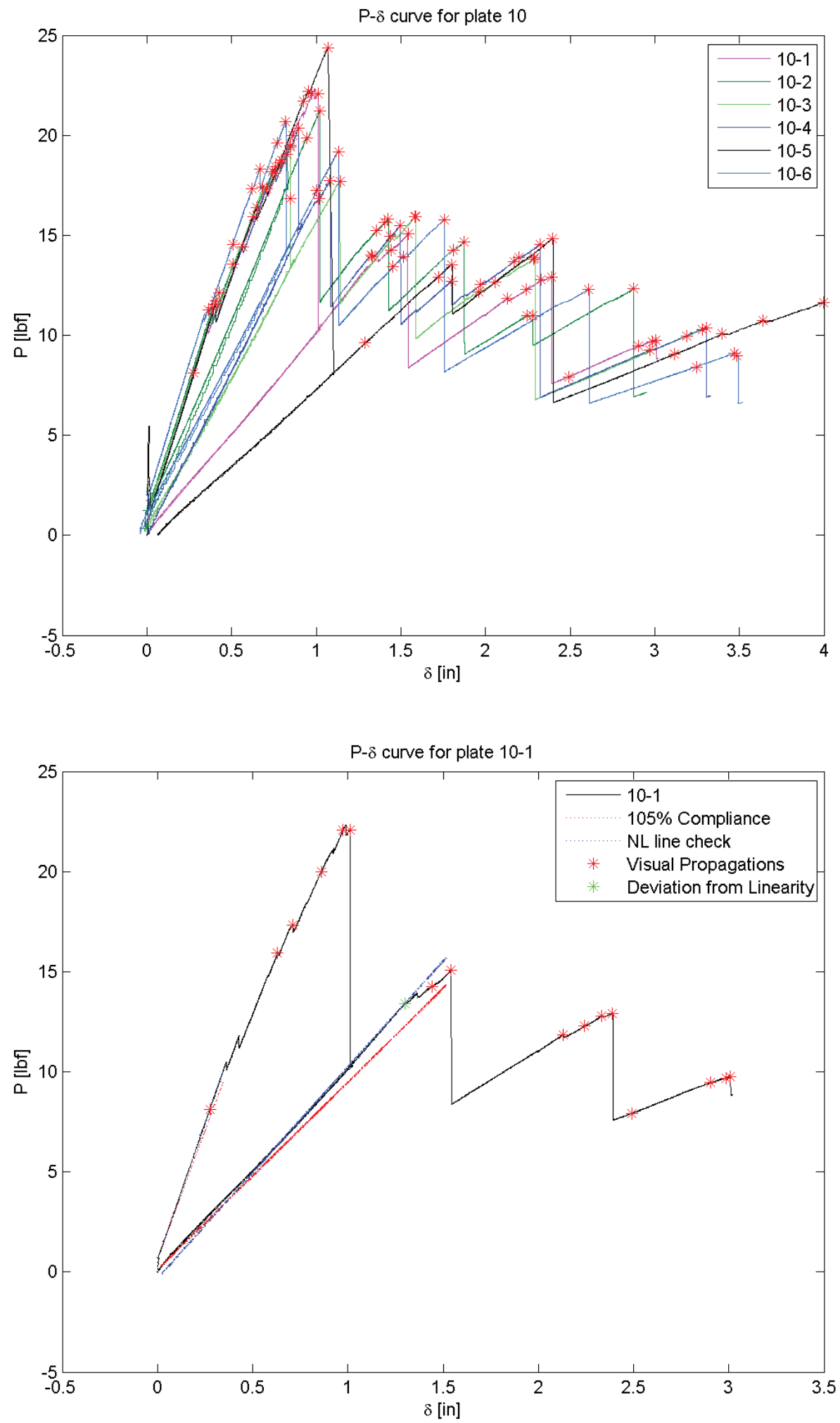

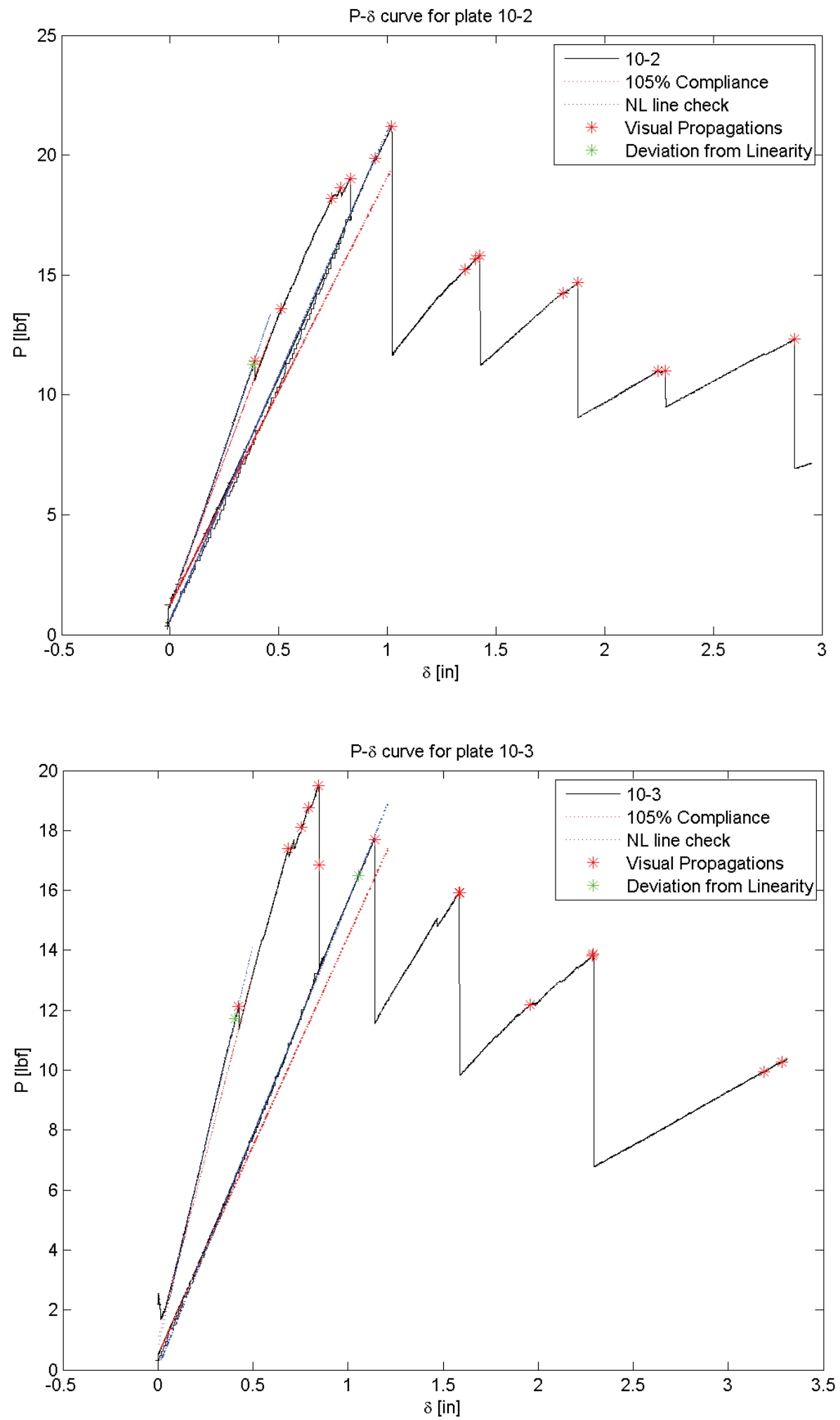

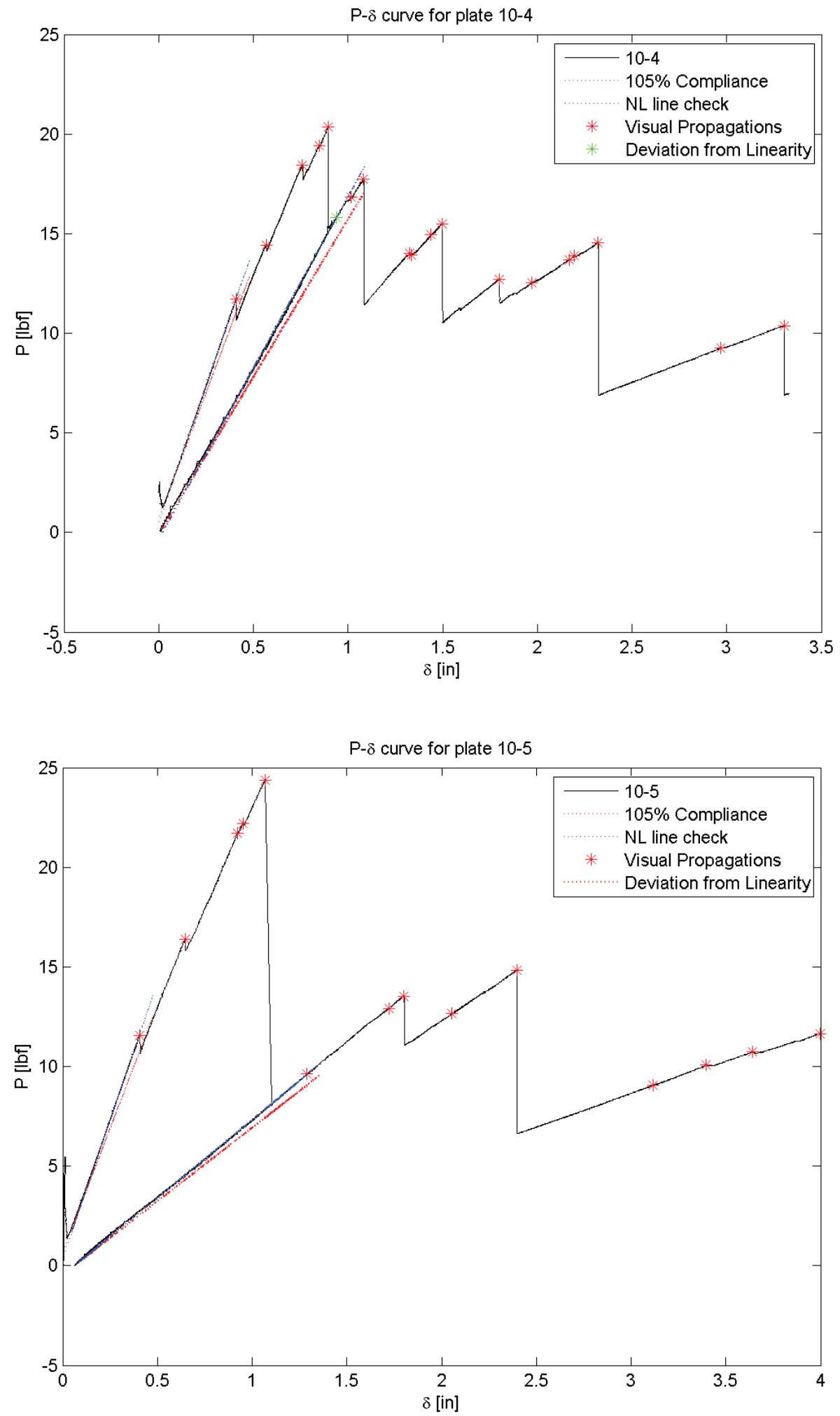


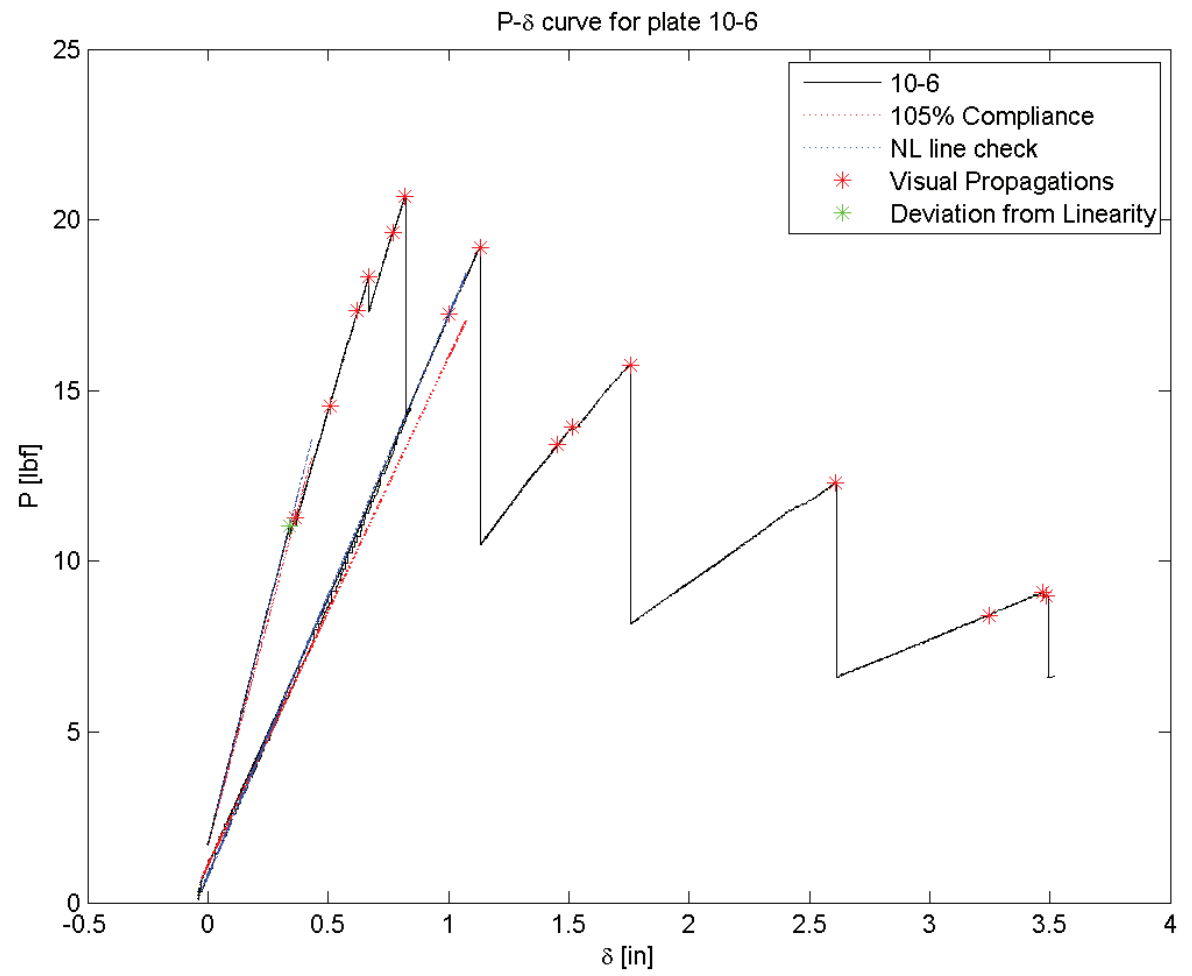


F.6 $P-\delta$ Plots for Plate 11
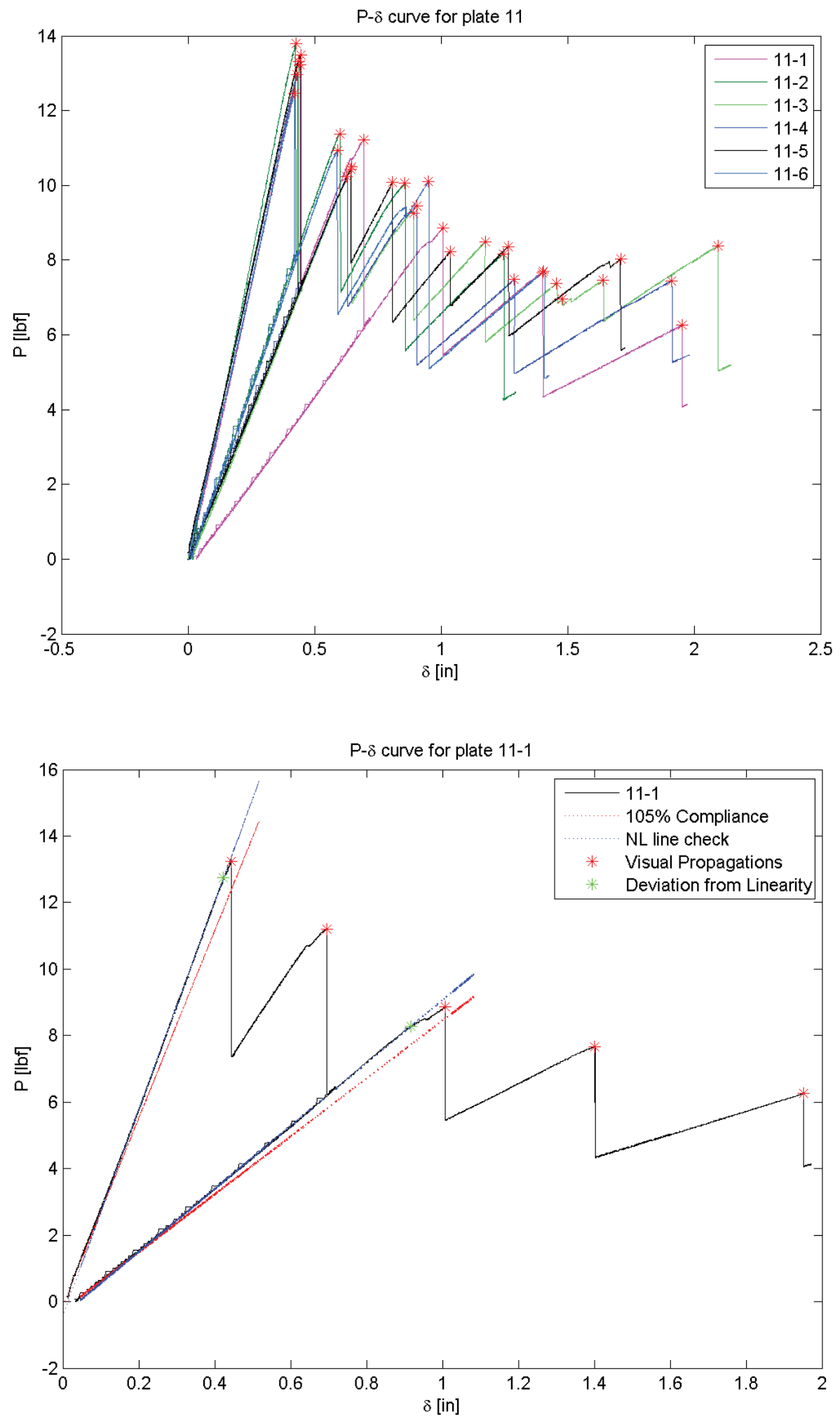

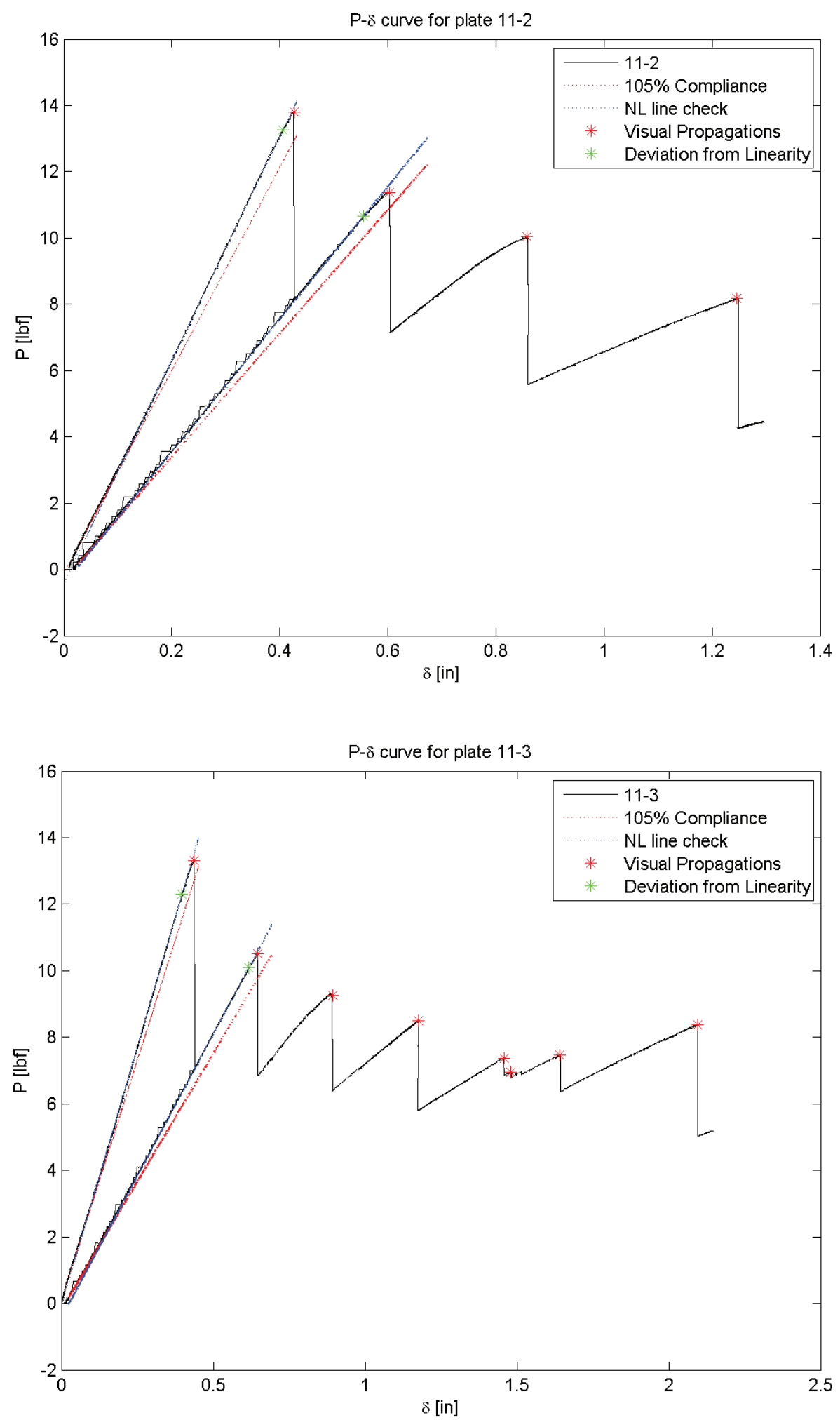

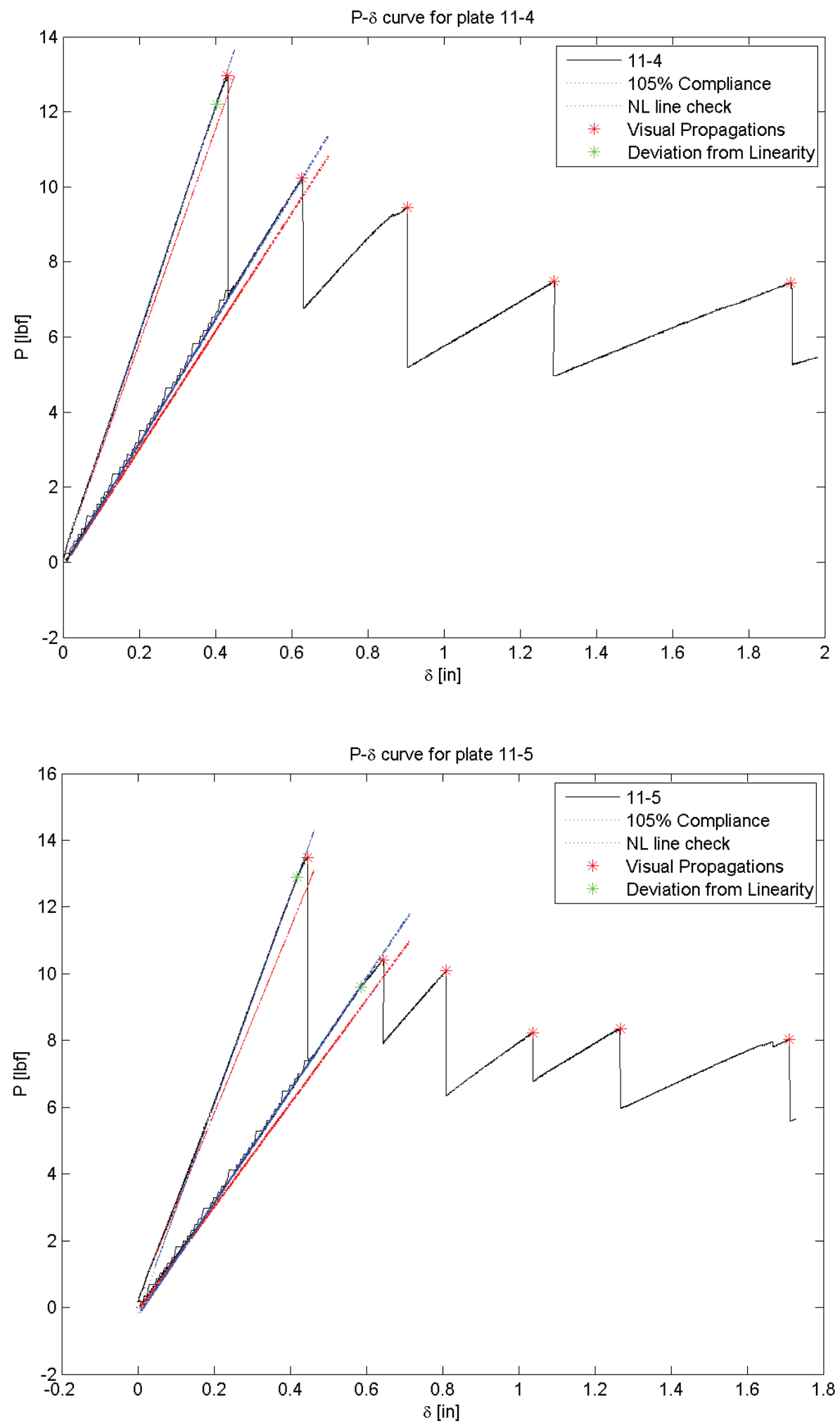


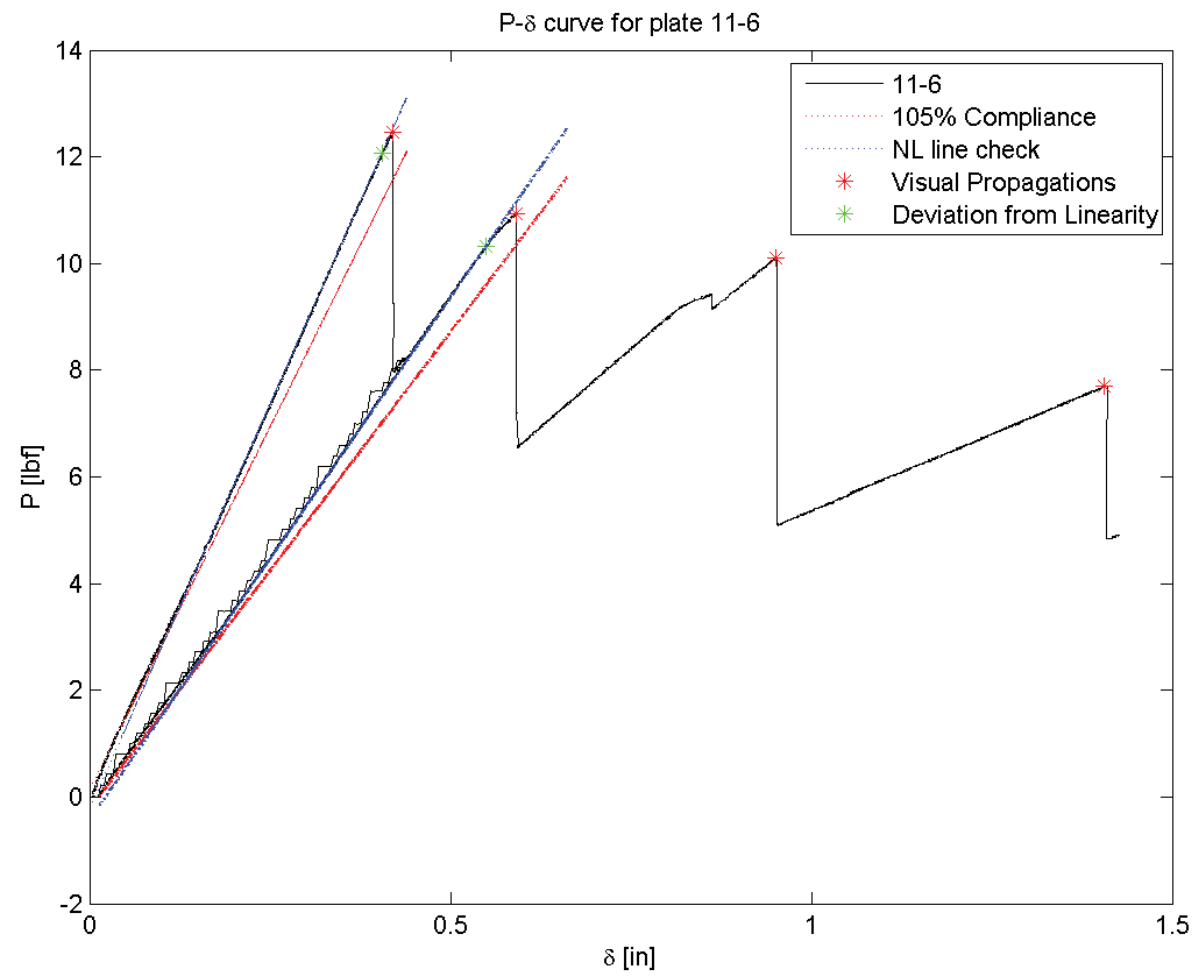




\begin{abstract}
AppendixG
DCB Specimen R-Curve Plots
\end{abstract}




\section{G.1 R-Curves for Plate 6}

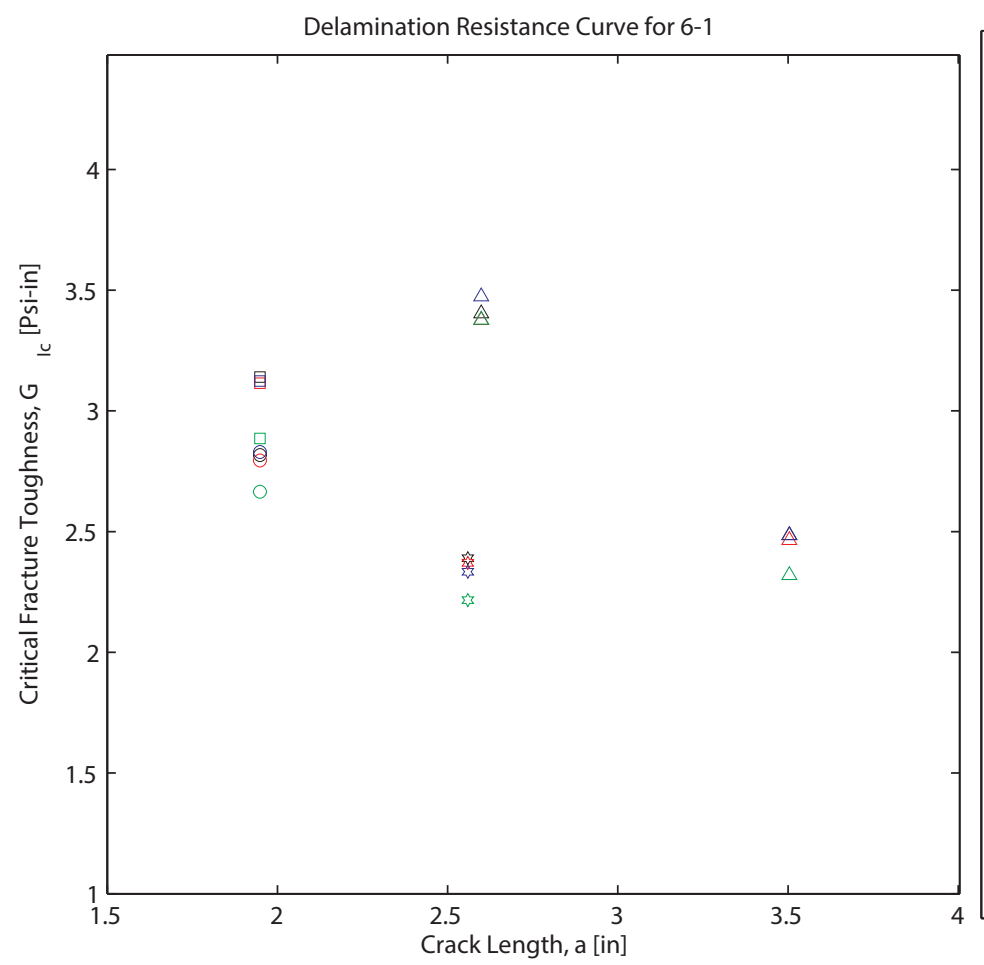

\begin{tabular}{|c|c|}
\hline$\square$ & Visual Onset from Insert -- MBT \\
\hline$\square$ & $\mathrm{CC}$ \\
\hline$\square$ & MCC \\
\hline$\square$ & EQS \\
\hline O & Deviation from Linearity at Insert -- MBT \\
\hline ○ & $\mathrm{CC}$ \\
\hline O & MCC \\
\hline O & EQS \\
\hline$\diamond$ & $5 \%$ Offset from Insert -- MBT \\
\hline$\diamond$ & $\mathrm{CC}$ \\
\hline$\diamond$ & MCC \\
\hline$\diamond$ & EQS \\
\hline 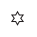 & Visual Onset from Precrack -- MBT \\
\hline \&3 & $\mathrm{CC}$ \\
\hline$\hat{3}$ & MCC \\
\hline is & EQS \\
\hline is & Deviation from Linearity at Precrack -- MBT \\
\hline it & $\mathrm{CC}$ \\
\hline it & MCC \\
\hline is & EQS \\
\hline$\times$ & $5 \%$ offset from Precrack-- MBT \\
\hline$x$ & $\mathrm{CC}$ \\
\hline$x$ & MCC \\
\hline$x$ & EQS \\
\hline$\triangle$ & Crack Propagation -- MBT \\
\hline$\triangle$ & $\mathrm{CC}$ \\
\hline$\triangle$ & MCC \\
\hline$\triangle$ & EQS \\
\hline
\end{tabular}

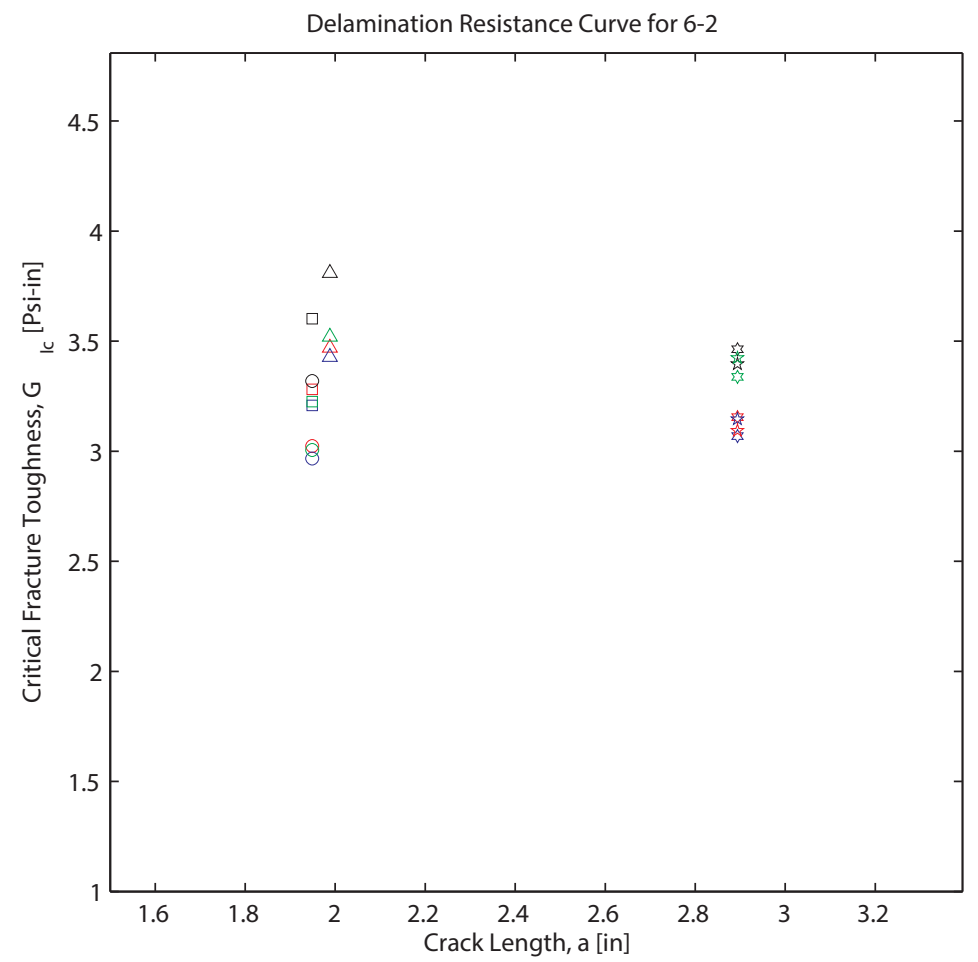

\begin{tabular}{|c|c|}
\hline$\square$ & Visual Onset from Insert -- MBT \\
\hline$\square$ & $\mathrm{CC}$ \\
\hline$\square$ & MCC \\
\hline$\square$ & EQS \\
\hline o & Deviation from Linearity at Insert -- MBT \\
\hline o & CC \\
\hline o & MCC \\
\hline o & EQS \\
\hline$\diamond$ & $5 \%$ Offset from Insert -- MBT \\
\hline$\diamond$ & $\mathrm{CC}$ \\
\hline$\diamond$ & MCC \\
\hline$\diamond$ & EQS \\
\hline 急 & Visual Onset from Precrack -- MBT \\
\hline 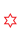 & $\mathrm{CC}$ \\
\hline 级 & MCC \\
\hline$\hat{3}$ & EQS \\
\hline is & Deviation from Linearity at Precrack -- MBT \\
\hline 出 & $\mathrm{CC}$ \\
\hline it & MCC \\
\hline मे & EQS \\
\hline$\times$ & $5 \%$ offset from Precrack-- MBT \\
\hline$x$ & $\mathrm{CC}$ \\
\hline$x$ & MCC \\
\hline$\times$ & EQS \\
\hline$\triangle$ & Crack Propagation -- MBT \\
\hline$\triangle$ & $\mathrm{CC}$ \\
\hline$\triangle$ & MCC \\
\hline$\triangle$ & EQS \\
\hline
\end{tabular}




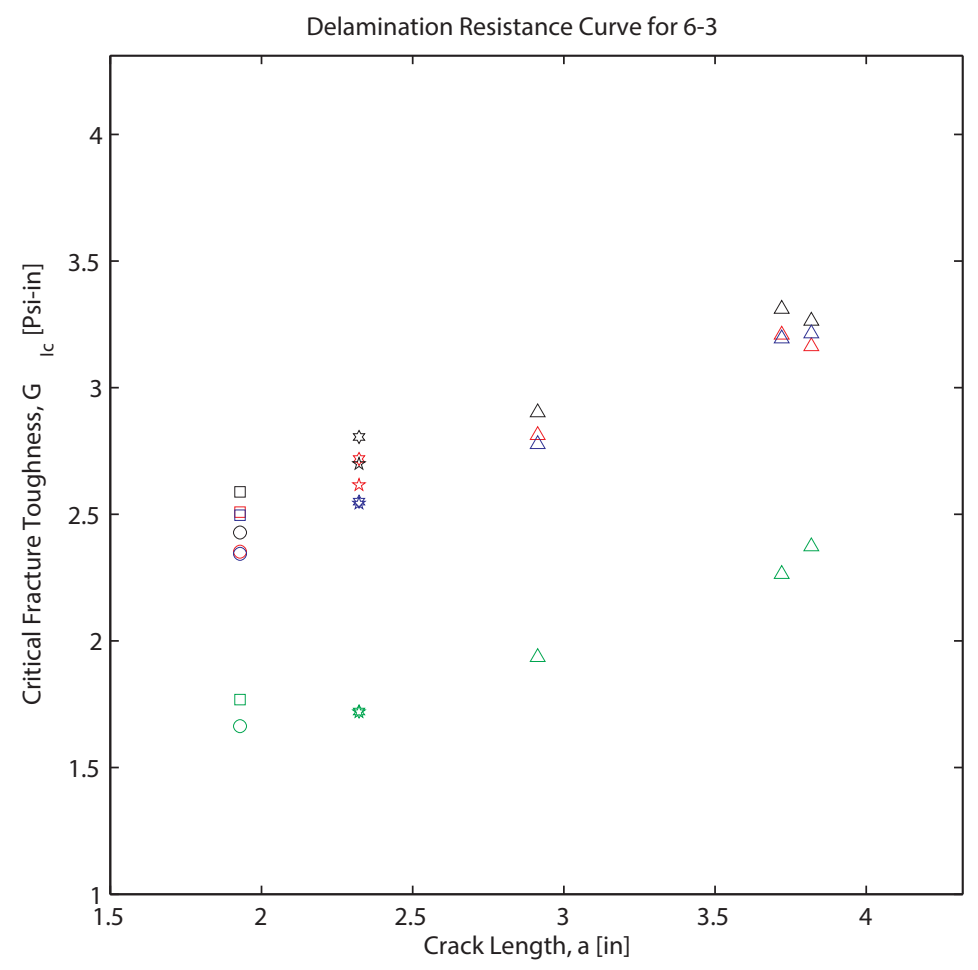

\begin{tabular}{|c|c|}
\hline$\square$ & Visual Onset from Insert -- MBT \\
\hline$\square$ & $\mathrm{CC}$ \\
\hline$\square$ & MCC \\
\hline$\square$ & EQS \\
\hline O & Deviation from Linearity at Insert -- MBT \\
\hline O & $\mathrm{CC}$ \\
\hline O & MCC \\
\hline O & EQS \\
\hline$\diamond$ & $5 \%$ Offset from Insert -- MBT \\
\hline$\diamond$ & $\mathrm{CC}$ \\
\hline$\diamond$ & MCC \\
\hline$\diamond$ & EQS \\
\hline 约 & Visual Onset from Precrack -- MBT \\
\hline s & $\mathrm{CC}$ \\
\hline 约 & MCC \\
\hline s & EQS \\
\hline 放 & Deviation from Linearity at Precrack -- MBT \\
\hline it & $\mathrm{CC}$ \\
\hline is & MCC \\
\hline it & EQS \\
\hline$\times$ & $5 \%$ offset from Precrack-- MBT \\
\hline$\times$ & $\mathrm{CC}$ \\
\hline$x$ & MCC \\
\hline$\times$ & EQS \\
\hline$\triangle$ & Crack Propagation -- MBT \\
\hline$\triangle$ & $\mathrm{CC}$ \\
\hline$\triangle$ & MCC \\
\hline$\triangle$ & EQS \\
\hline
\end{tabular}

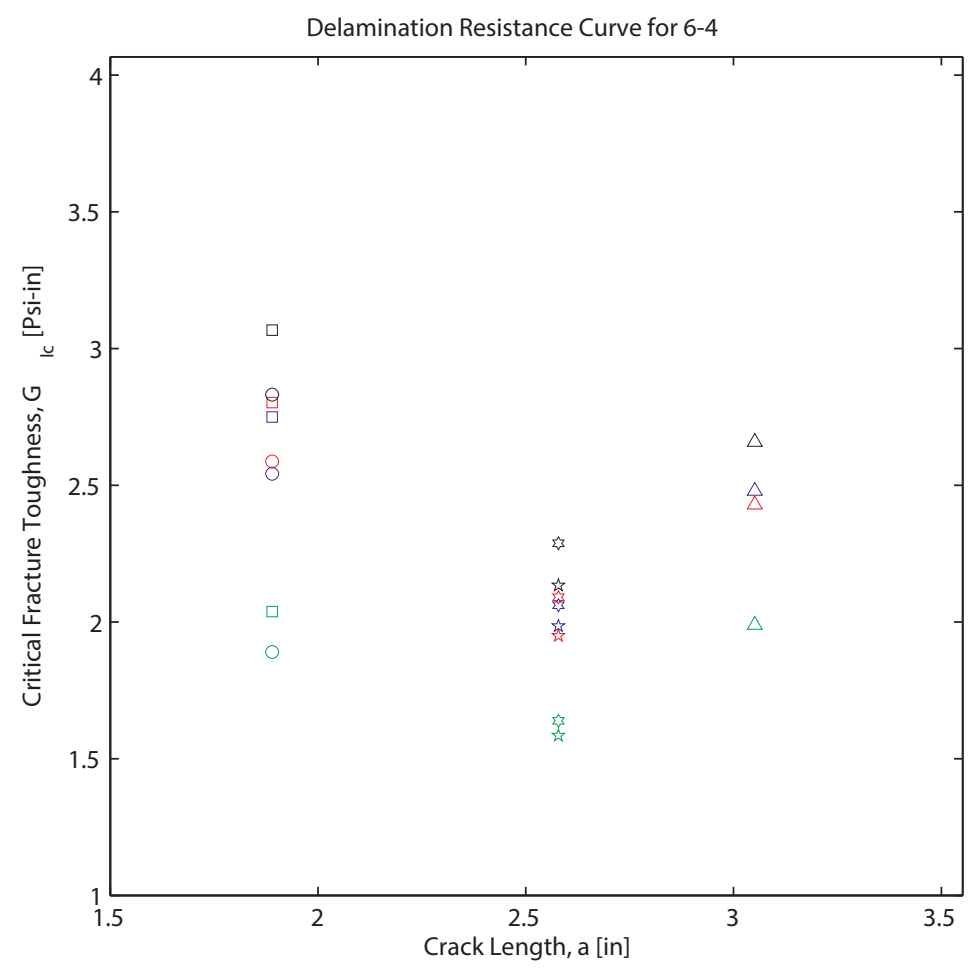

\begin{tabular}{|c|c|}
\hline$\square$ & Visual Onset from Insert -- MBT \\
\hline$\square$ & $\mathrm{CC}$ \\
\hline$\square$ & MCC \\
\hline$\square$ & EQS \\
\hline O & Deviation from Linearity at Insert -- MBT \\
\hline o & $\mathrm{CC}$ \\
\hline o & MCC \\
\hline o & EQS \\
\hline$\diamond$ & $5 \%$ Offset from Insert -- MBT \\
\hline$\diamond$ & $\mathrm{CC}$ \\
\hline$\diamond$ & MCC \\
\hline$\diamond$ & EQS \\
\hline s & Visual Onset from Precrack -- MBT \\
\hline 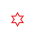 & $\mathrm{CC}$ \\
\hline 皮 & MCC \\
\hline s & EQS \\
\hline it & Deviation from Linearity at Precrack -- MBT \\
\hline 解 & $\mathrm{CC}$ \\
\hline 论 & MCC \\
\hline 实 & EQS \\
\hline$x$ & $5 \%$ offset from Precrack-- MBT \\
\hline$\times$ & $\mathrm{CC}$ \\
\hline$x$ & MCC \\
\hline$x$ & EQS \\
\hline$\triangle$ & Crack Propagation -- MBT \\
\hline$\triangle$ & $\mathrm{CC}$ \\
\hline$\triangle$ & MCC \\
\hline$\triangle$ & EQS \\
\hline
\end{tabular}




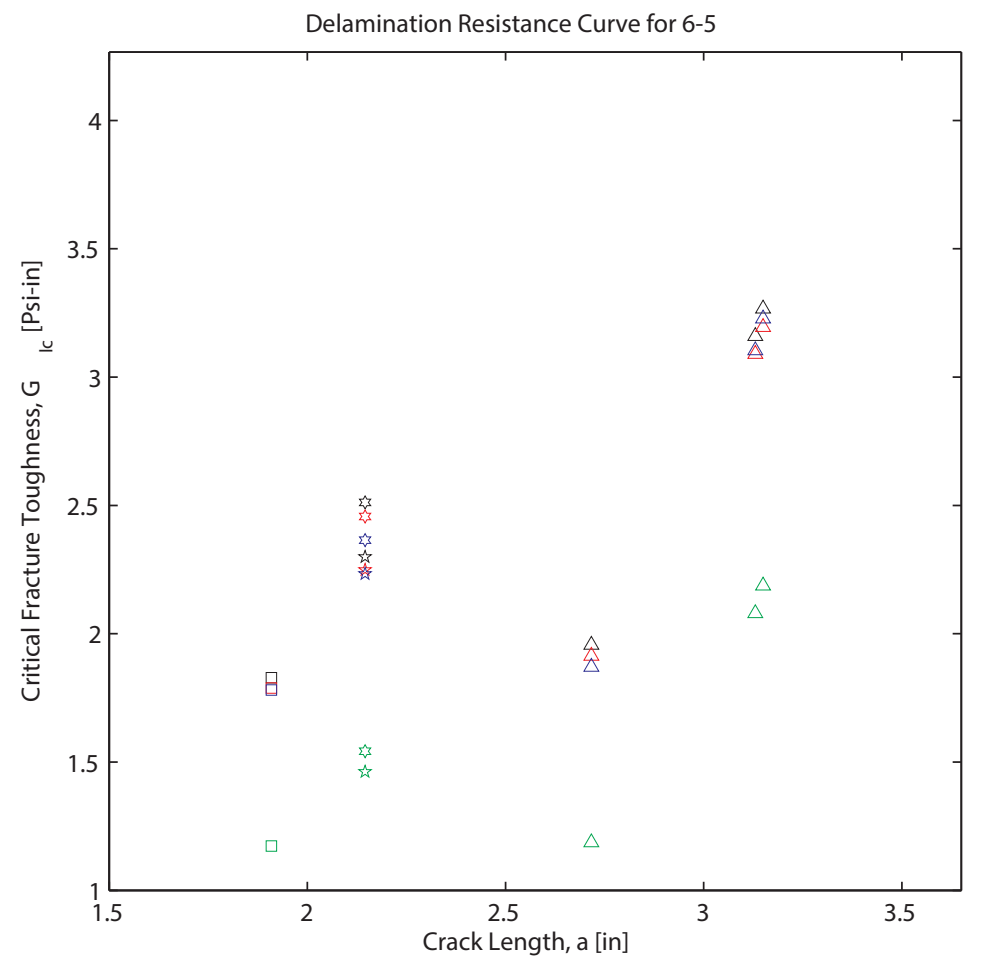

\begin{tabular}{|c|c|}
\hline$\square$ & Visual Onset from Insert -- MBT \\
\hline$\square$ & $\mathrm{CC}$ \\
\hline$\square$ & MCC \\
\hline$\square$ & EQS \\
\hline ○ & Deviation from Linearity at Insert -- MBT \\
\hline O & $\mathrm{CC}$ \\
\hline O & MCC \\
\hline ○ & EQS \\
\hline$\diamond$ & $5 \%$ Offset from Insert -- MBT \\
\hline$\diamond$ & $\mathrm{CC}$ \\
\hline$\diamond$ & MCC \\
\hline$\diamond$ & EQS \\
\hline 级 & Visual Onset from Precrack -- MBT \\
\hline 3 & $\mathrm{CC}$ \\
\hline 级 & MCC \\
\hline 3 & EQS \\
\hline is & Deviation from Linearity at Precrack -- MBT \\
\hline it & $\mathrm{CC}$ \\
\hline it & MCC \\
\hline is & EQS \\
\hline$\times$ & $5 \%$ offset from Precrack-- MBT \\
\hline$\times$ & $\mathrm{CC}$ \\
\hline$\times$ & MCC \\
\hline$\times$ & EQS \\
\hline$\triangle$ & Crack Propagation -- MBT \\
\hline$\triangle$ & $\mathrm{CC}$ \\
\hline$\triangle$ & MCC \\
\hline$\triangle$ & EQS \\
\hline
\end{tabular}

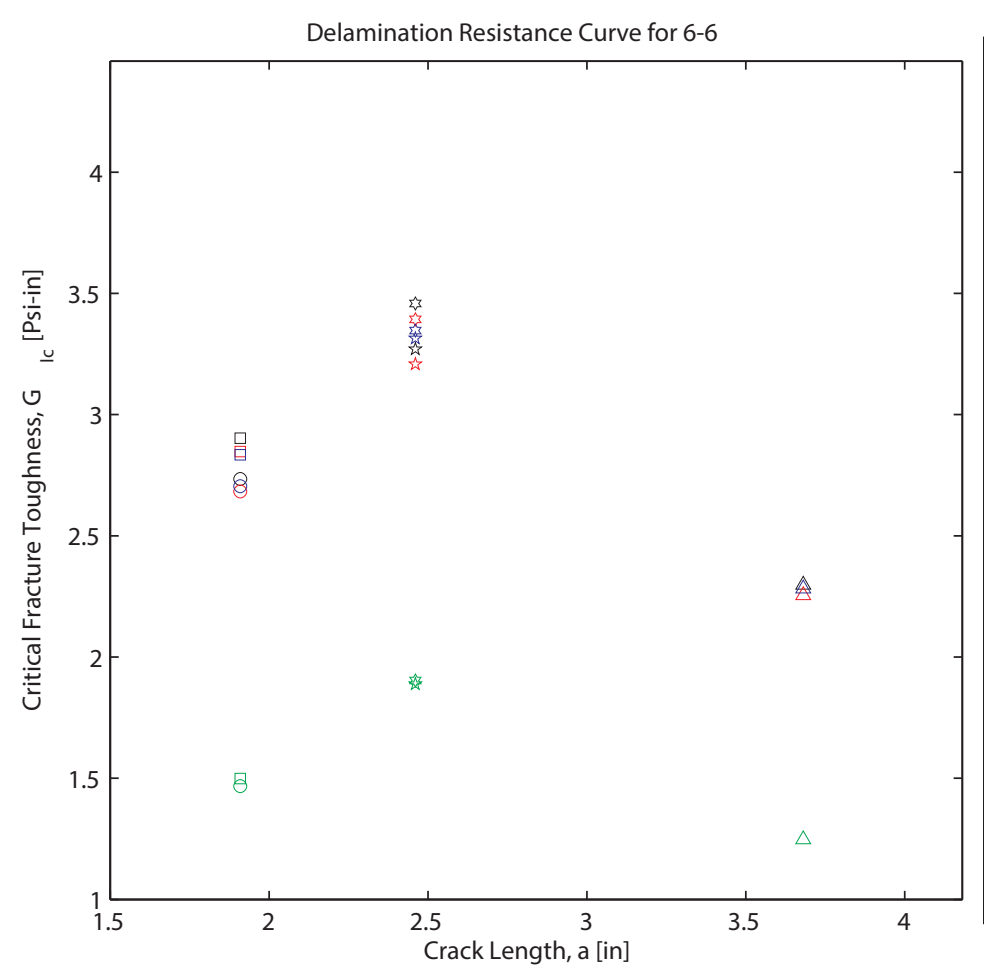

\begin{tabular}{|c|c|}
\hline$\square$ & Visual Onset from Insert -- MBT \\
\hline$\square$ & $\mathrm{CC}$ \\
\hline$\square$ & MCC \\
\hline$\square$ & EQS \\
\hline O & Deviation from Linearity at Insert -- MBT \\
\hline O & $\mathrm{CC}$ \\
\hline O & MCC \\
\hline O & EQS \\
\hline$\diamond$ & $5 \%$ Offset from Insert -- MBT \\
\hline$\diamond$ & $\mathrm{CC}$ \\
\hline$\diamond$ & MCC \\
\hline$\diamond$ & EQS \\
\hline is & Visual Onset from Precrack -- MBT \\
\hline 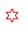 & $\mathrm{CC}$ \\
\hline is & MCC \\
\hline 3 & EQS \\
\hline is & Deviation from Linearity at Precrack -- MBT \\
\hline is & $\mathrm{CC}$ \\
\hline it & MCC \\
\hline it & EQS \\
\hline$x$ & $5 \%$ offset from Precrack-- MBT \\
\hline$\times$ & $\mathrm{CC}$ \\
\hline$x$ & MCC \\
\hline$x$ & EQS \\
\hline$\triangle$ & Crack Propagation -- MBT \\
\hline$\triangle$ & $\mathrm{CC}$ \\
\hline$\triangle$ & MCC \\
\hline$\triangle$ & EQS \\
\hline
\end{tabular}




\section{G.2 R-Curves for Plate 7}
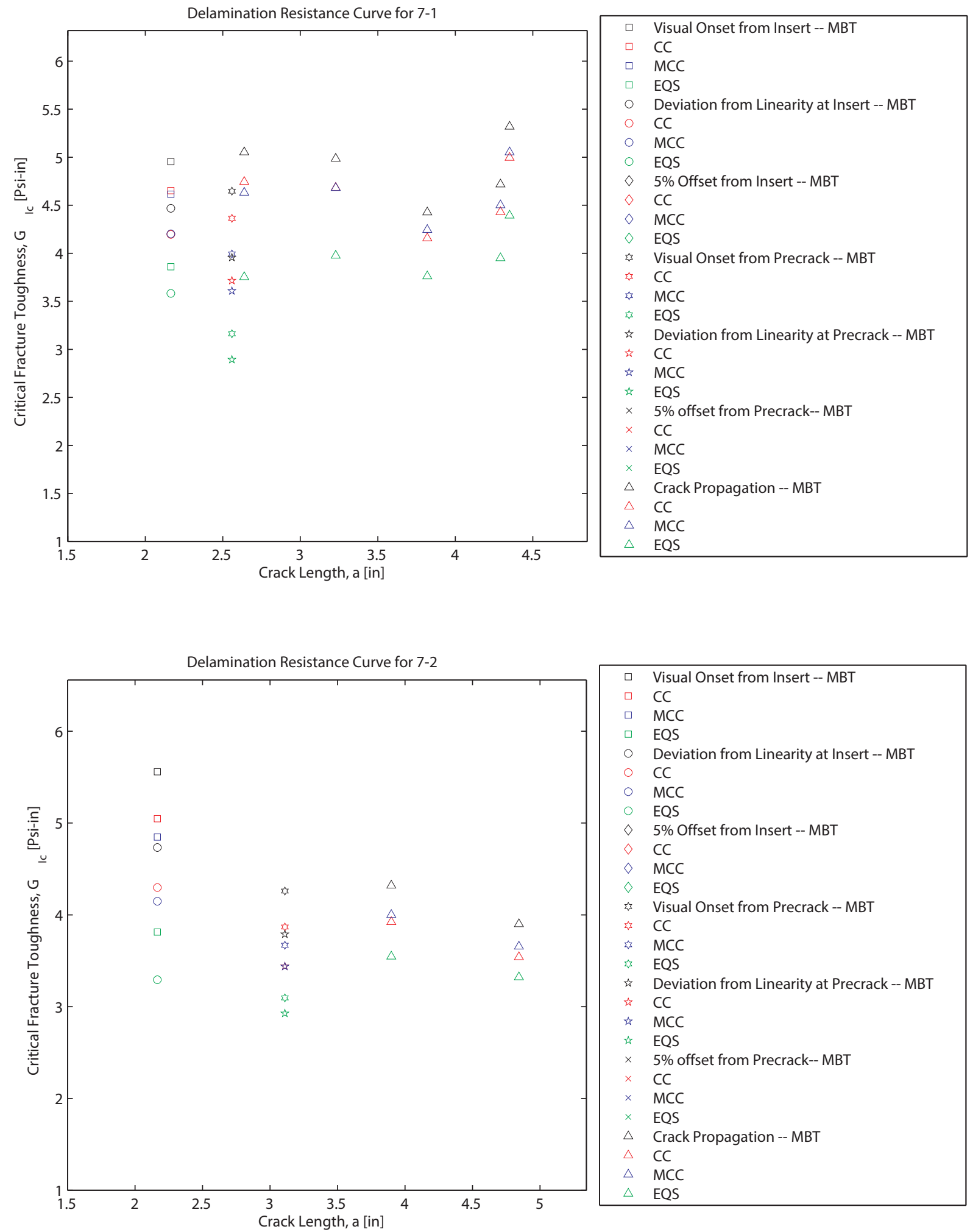


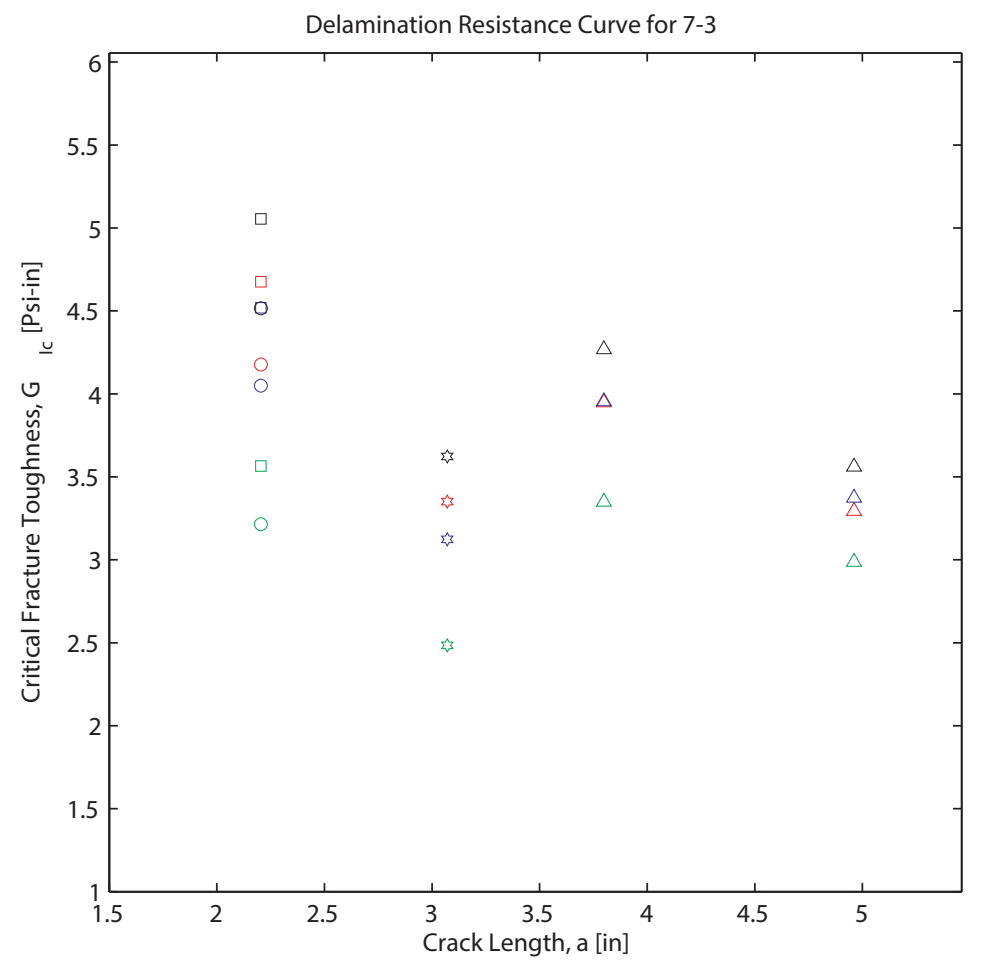

\begin{tabular}{|c|c|}
\hline$\square$ & Visual Onset from Insert -- MBT \\
\hline$\square$ & $\mathrm{CC}$ \\
\hline$\square$ & MCC \\
\hline$\square$ & EQS \\
\hline O & Deviation from Linearity at Insert -- MBT \\
\hline O & $\mathrm{CC}$ \\
\hline O & MCC \\
\hline O & EQS \\
\hline$\diamond$ & $5 \%$ Offset from Insert -- MBT \\
\hline$\diamond$ & $\mathrm{CC}$ \\
\hline$\diamond$ & MCC \\
\hline$\diamond$ & EQS \\
\hline$\hat{s}$ & Visual Onset from Precrack -- MBT \\
\hline 3 & $\mathrm{CC}$ \\
\hline$\hat{s}$ & MCC \\
\hline 3 & EQS \\
\hline is & Deviation from Linearity at Precrack -- MBT \\
\hline is & $\mathrm{CC}$ \\
\hline 访 & MCC \\
\hline 放 & EQS \\
\hline$x$ & $5 \%$ offset from Precrack-- MBT \\
\hline$x$ & $\mathrm{CC}$ \\
\hline$x$ & MCC \\
\hline$x$ & EQS \\
\hline$\triangle$ & Crack Propagation -- MBT \\
\hline$\triangle$ & $\mathrm{CC}$ \\
\hline$\triangle$ & MCC \\
\hline$\triangle$ & EQS \\
\hline
\end{tabular}

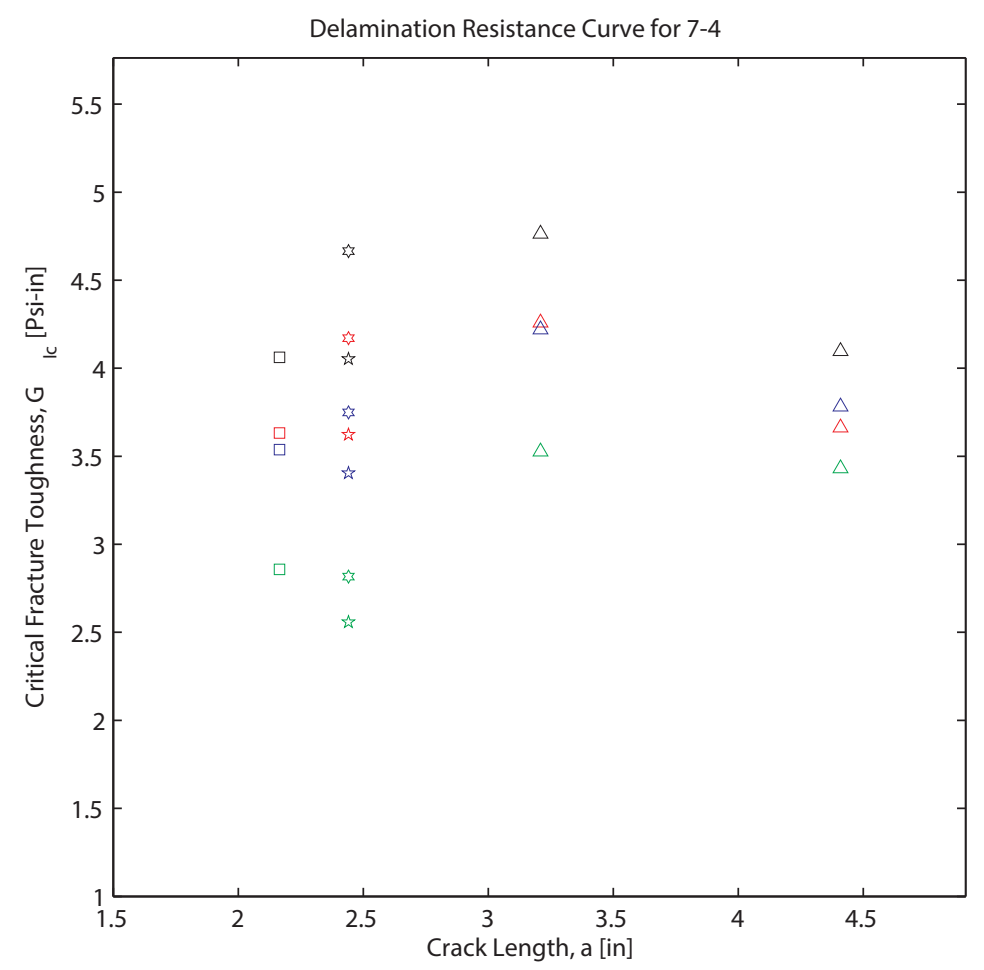

\begin{tabular}{|c|c|}
\hline$\square$ & Visual Onset from Insert -- MBT \\
\hline$\square$ & $\mathrm{CC}$ \\
\hline$\square$ & MCC \\
\hline$\square$ & EQS \\
\hline ○ & Deviation from Linearity at Insert -- MBT \\
\hline o & $\mathrm{CC}$ \\
\hline o & MCC \\
\hline O & EQS \\
\hline$\diamond$ & $5 \%$ Offset from Insert -- MBT \\
\hline$\diamond$ & $\mathrm{CC}$ \\
\hline$\diamond$ & MCC \\
\hline$\diamond$ & EQS \\
\hline 3 & Visual Onset from Precrack -- MBT \\
\hline$\Leftrightarrow$ & $\mathrm{CC}$ \\
\hline 级 & MCC \\
\hline s & EQS \\
\hline it & Deviation from Linearity at Precrack -- MBT \\
\hline 解 & $\mathrm{CC}$ \\
\hline 论 & MCC \\
\hline is & EQS \\
\hline$x$ & $5 \%$ offset from Precrack-- MBT \\
\hline$\times$ & $\mathrm{CC}$ \\
\hline$x$ & MCC \\
\hline$x$ & EQS \\
\hline$\triangle$ & Crack Propagation -- MBT \\
\hline$\triangle$ & $\mathrm{CC}$ \\
\hline$\triangle$ & MCC \\
\hline$\triangle$ & EQS \\
\hline
\end{tabular}




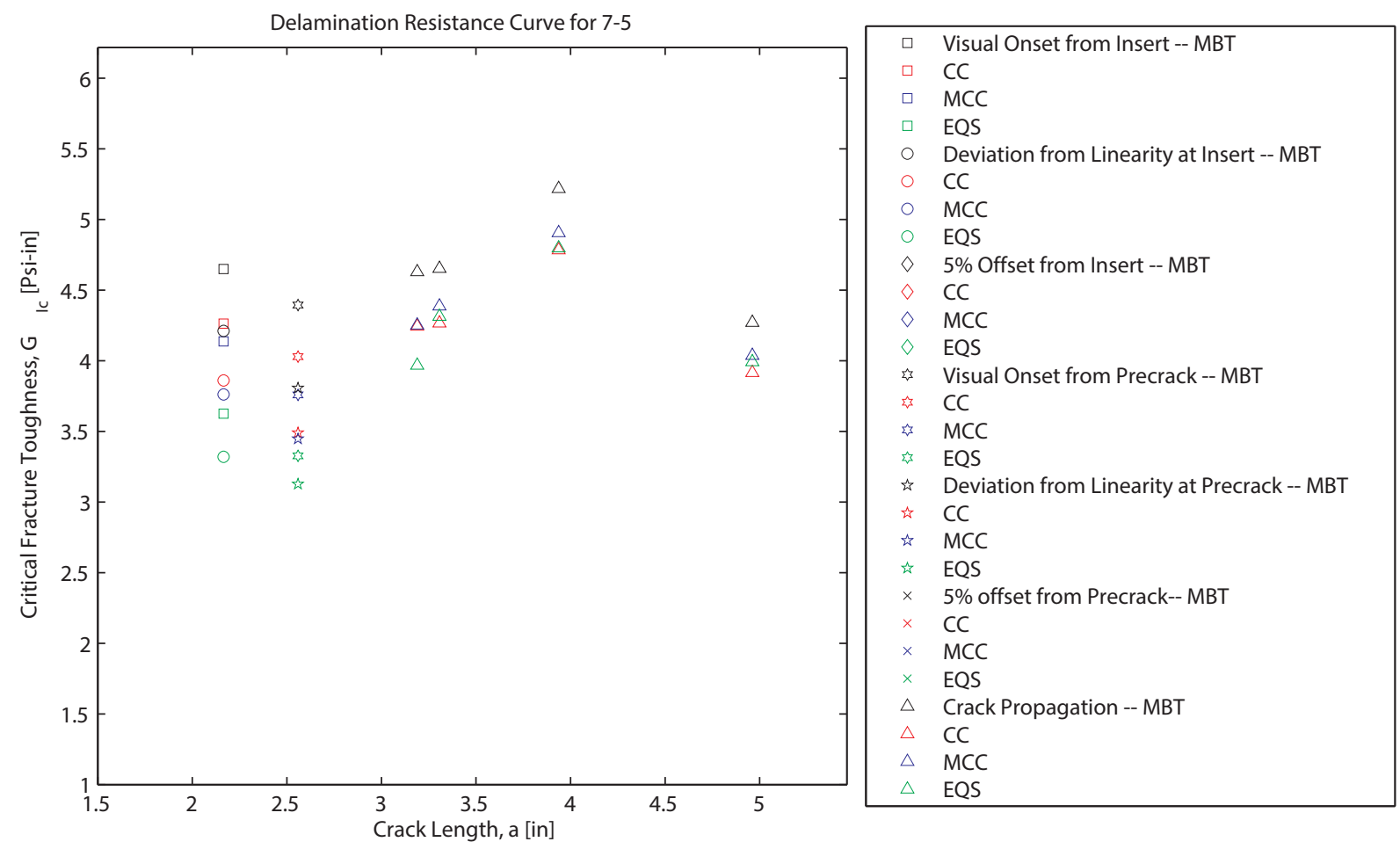




\section{G.3 R-Curves for Plate 8}

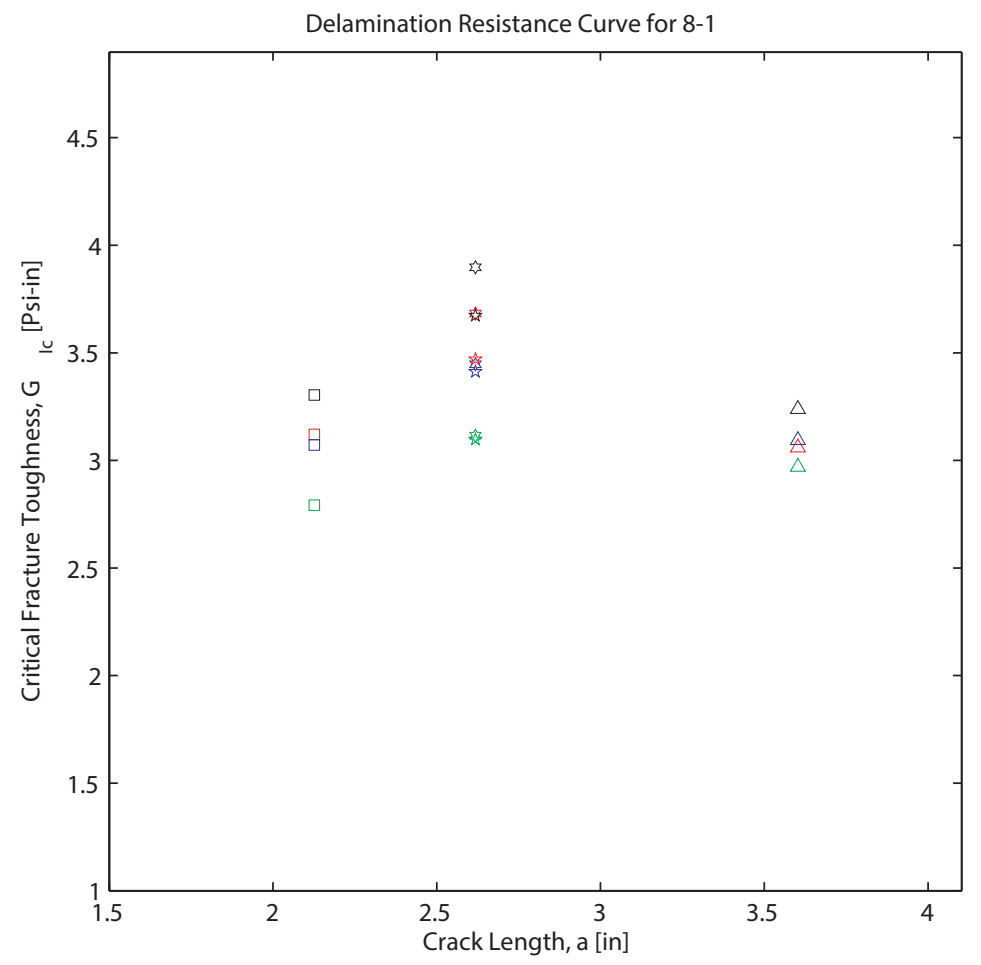

\begin{tabular}{|c|c|}
\hline$\square$ & Visual Onset from Insert -- MBT \\
\hline$\square$ & $\mathrm{CC}$ \\
\hline$\square$ & MCC \\
\hline$\square$ & EQS \\
\hline ○ & Deviation from Linearity at Insert -- MBT \\
\hline o & $\mathrm{CC}$ \\
\hline o & MCC \\
\hline o & EQS \\
\hline$\diamond$ & $5 \%$ Offset from Insert -- MBT \\
\hline$\diamond$ & $\mathrm{CC}$ \\
\hline$\diamond$ & MCC \\
\hline$\diamond$ & EQS \\
\hline$\hat{s}^{2}$ & Visual Onset from Precrack -- MBT \\
\hline \& & $\mathrm{CC}$ \\
\hline 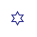 & MCC \\
\hline is & EQS \\
\hline 类 & Deviation from Linearity at Precrack -- MBT \\
\hline is & $\mathrm{CC}$ \\
\hline is & MCC \\
\hline 它 & EQS \\
\hline$x$ & $5 \%$ offset from Precrack-- MBT \\
\hline$x$ & $\mathrm{CC}$ \\
\hline$\times$ & MCC \\
\hline$x$ & EQS \\
\hline$\triangle$ & Crack Propagation -- MBT \\
\hline$\triangle$ & $\mathrm{CC}$ \\
\hline$\triangle$ & MCC \\
\hline$\triangle$ & EQS \\
\hline
\end{tabular}

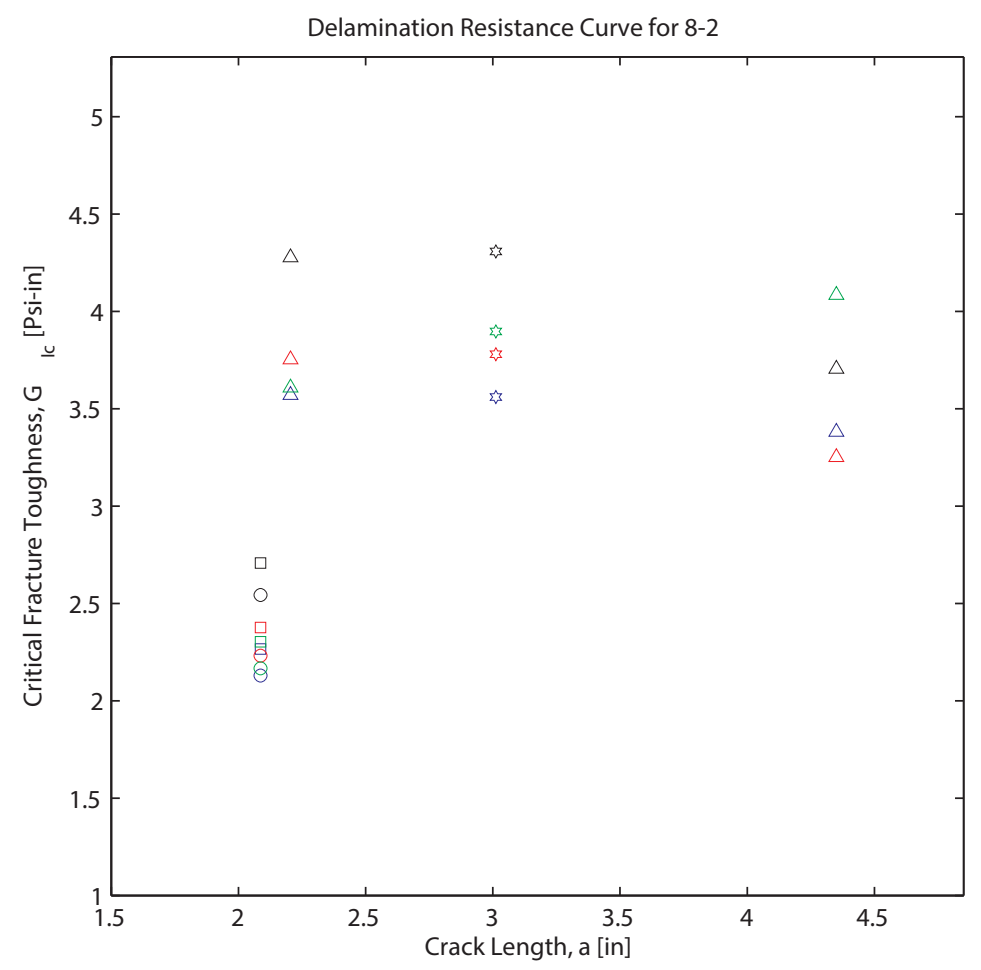

\begin{tabular}{|c|c|}
\hline 口 & Visual Onset from Insert -- MBT \\
\hline 口 & $\mathrm{CC}$ \\
\hline$\square$ & MCC \\
\hline 口 & EQS \\
\hline 0 & Deviation from Linearity at Insert -- MBT \\
\hline 0 & CC \\
\hline 0 & MCC \\
\hline 0 & EQS \\
\hline$\diamond$ & $5 \%$ Offset from Insert -- MBT \\
\hline$\diamond$ & CC \\
\hline$\diamond$ & MCC \\
\hline$\diamond$ & EQS \\
\hline 级 & Visual Onset from Precrack -- MBT \\
\hline 级 & CC \\
\hline 边 & MCC \\
\hline \& & EQS \\
\hline is & Deviation from Linearity at Precrack -- MBT \\
\hline is & CC \\
\hline is & MCC \\
\hline is & EQS \\
\hline$x$ & $5 \%$ offset from Precrack-- MBT \\
\hline$x$ & $\mathrm{CC}$ \\
\hline$x$ & MCC \\
\hline$x$ & EQS \\
\hline$\Delta$ & Crack Propagation -- MBT \\
\hline$\Delta$ & $\mathrm{CC}$ \\
\hline$\Delta$ & MCC \\
\hline$\triangle$ & EQS \\
\hline
\end{tabular}




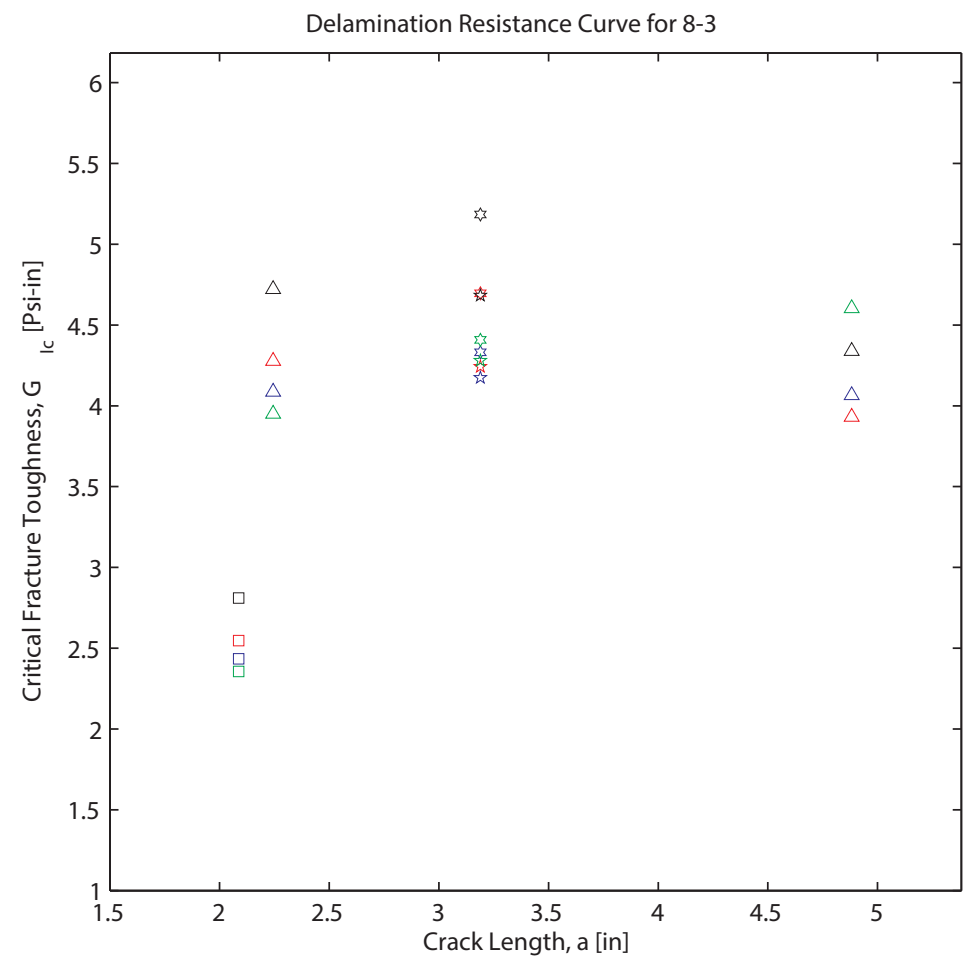

\begin{tabular}{|c|c|}
\hline$\square$ & Visual Onset from Insert -- MBT \\
\hline$\square$ & $\mathrm{CC}$ \\
\hline$\square$ & $\mathrm{MCC}$ \\
\hline$\square$ & EQS \\
\hline O & Deviation from Linearity at Insert -- MBT \\
\hline O & $\mathrm{CC}$ \\
\hline O & MCC \\
\hline O & EQS \\
\hline$\diamond$ & $5 \%$ Offset from Insert -- MBT \\
\hline$\diamond$ & $\mathrm{CC}$ \\
\hline$\diamond$ & MCC \\
\hline$\diamond$ & EQS \\
\hline 级 & Visual Onset from Precrack -- MBT \\
\hline$\xi_{3}$ & $\mathrm{CC}$ \\
\hline 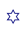 & MCC \\
\hline s & EQS \\
\hline is & Deviation from Linearity at Precrack -- MBT \\
\hline is & $\mathrm{CC}$ \\
\hline 幽 & MCC \\
\hline is & EQS \\
\hline$\times$ & $5 \%$ offset from Precrack-- MBT \\
\hline$\times$ & $\mathrm{CC}$ \\
\hline$\times$ & MCC \\
\hline$\times$ & EQS \\
\hline$\triangle$ & Crack Propagation -- MBT \\
\hline$\triangle$ & $\mathrm{CC}$ \\
\hline$\triangle$ & MCC \\
\hline$\triangle$ & EQS \\
\hline
\end{tabular}

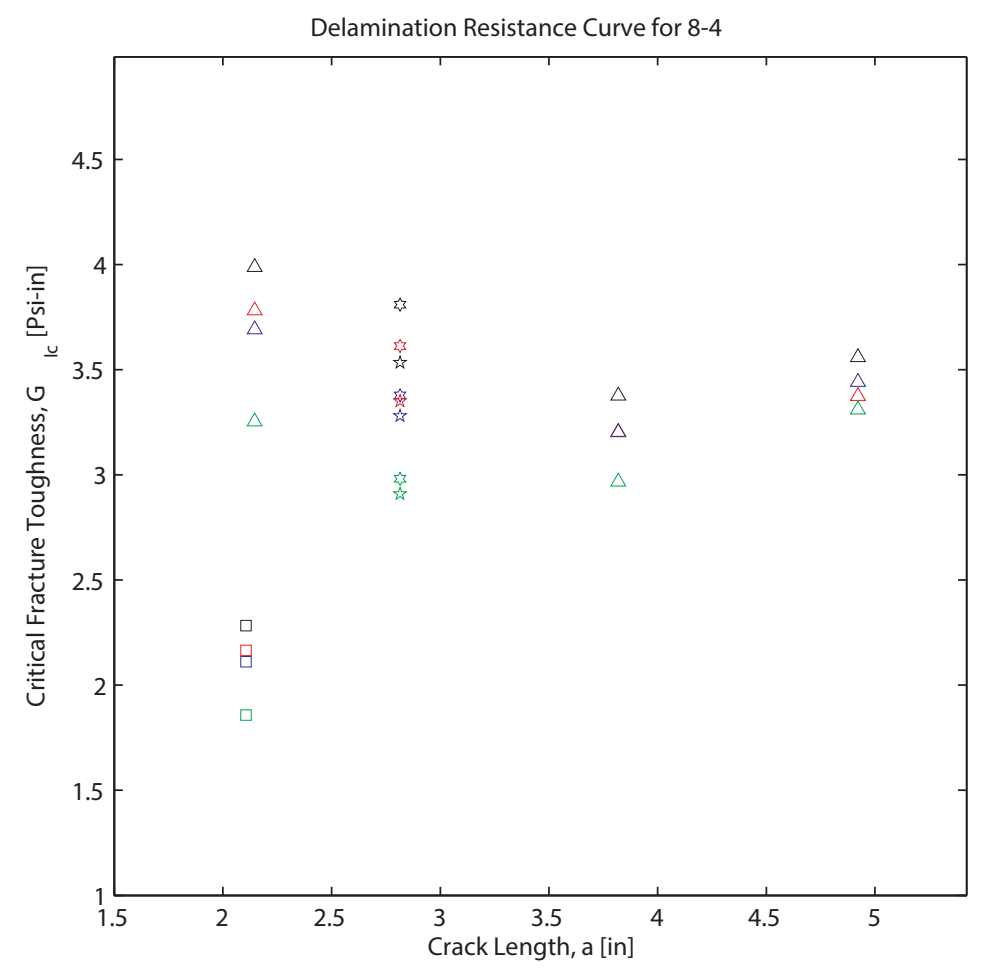

\begin{tabular}{|c|c|}
\hline$\square$ & Visual Onset from Insert -- MBT \\
\hline$\square$ & $\mathrm{CC}$ \\
\hline$\square$ & MCC \\
\hline$\square$ & EQS \\
\hline o & Deviation from Linearity at Insert -- MBT \\
\hline o & $\mathrm{CC}$ \\
\hline o & MCC \\
\hline O & EQS \\
\hline$\diamond$ & $5 \%$ Offset from Insert -- MBT \\
\hline$\diamond$ & $\mathrm{CC}$ \\
\hline$\diamond$ & MCC \\
\hline$\diamond$ & EQS \\
\hline 约 & Visual Onset from Precrack -- MBT \\
\hline$\Leftrightarrow$ & $\mathrm{CC}$ \\
\hline 约 & MCC \\
\hline$\xi$ & EQS \\
\hline it & Deviation from Linearity at Precrack -- MBT \\
\hline is & $\mathrm{CC}$ \\
\hline it & MCC \\
\hline is & EQS \\
\hline$\times$ & $5 \%$ offset from Precrack-- MBT \\
\hline$\times$ & $\mathrm{CC}$ \\
\hline$x$ & MCC \\
\hline$x$ & EQS \\
\hline$\triangle$ & Crack Propagation -- MBT \\
\hline$\triangle$ & $\mathrm{CC}$ \\
\hline$\triangle$ & MCC \\
\hline$\triangle$ & EQS \\
\hline
\end{tabular}




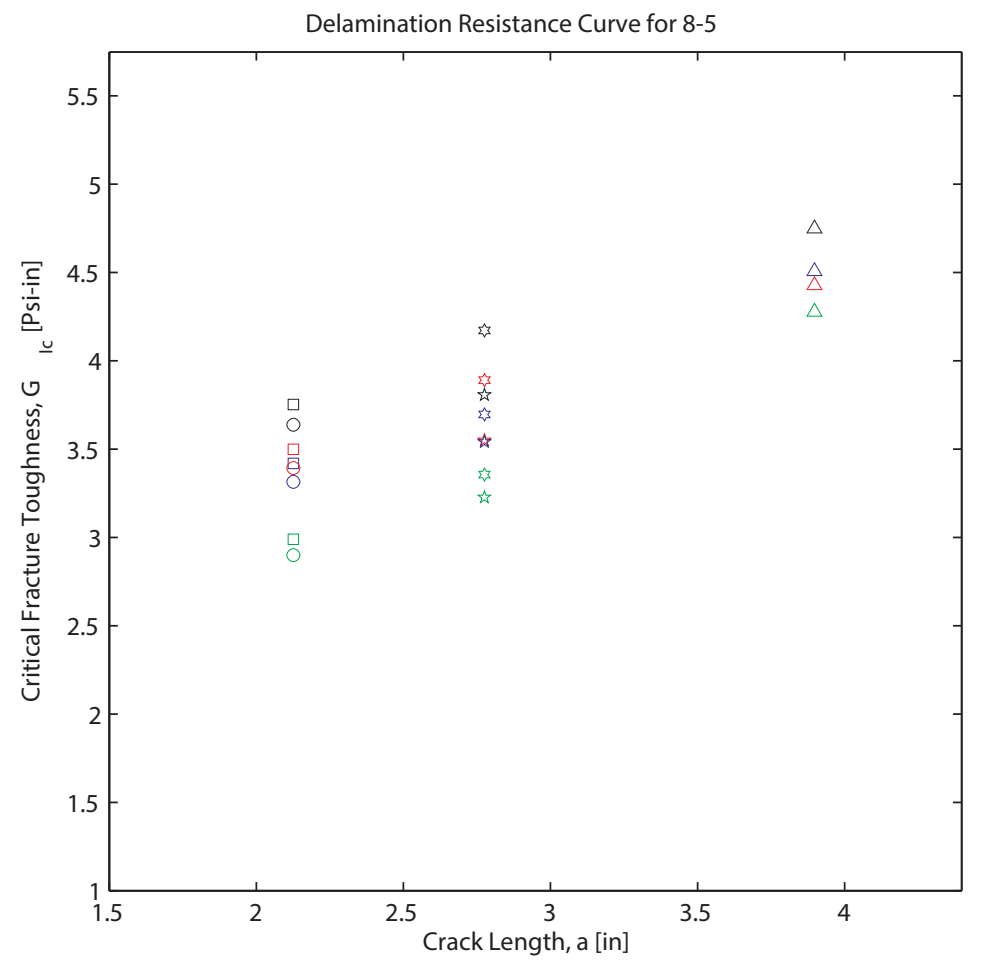

\begin{tabular}{|c|c|}
\hline$\square$ & Visual Onset from Insert -- MBT \\
\hline$\square$ & $\mathrm{CC}$ \\
\hline$\square$ & MCC \\
\hline$\square$ & EQS \\
\hline O & Deviation from Linearity at Insert -- MBT \\
\hline O & $\mathrm{CC}$ \\
\hline O & MCC \\
\hline O & EQS \\
\hline$\diamond$ & $5 \%$ Offset from Insert -- MBT \\
\hline$\diamond$ & $\mathrm{CC}$ \\
\hline$\diamond$ & MCC \\
\hline$\diamond$ & EQS \\
\hline$\hat{3}$ & Visual Onset from Precrack -- MBT \\
\hline 3 & CC \\
\hline$\hat{s}$ & MCC \\
\hline 3 & EQS \\
\hline is & Deviation from Linearity at Precrack -- MBT \\
\hline 约 & $\mathrm{CC}$ \\
\hline is & $\mathrm{MCC}$ \\
\hline 弥 & EQS \\
\hline$\times$ & $5 \%$ offset from Precrack-- MBT \\
\hline$\times$ & $\mathrm{CC}$ \\
\hline$\times$ & MCC \\
\hline$\times$ & EQS \\
\hline$\triangle$ & Crack Propagation -- MBT \\
\hline$\triangle$ & $\mathrm{CC}$ \\
\hline$\triangle$ & MCC \\
\hline$\triangle$ & EQS \\
\hline
\end{tabular}

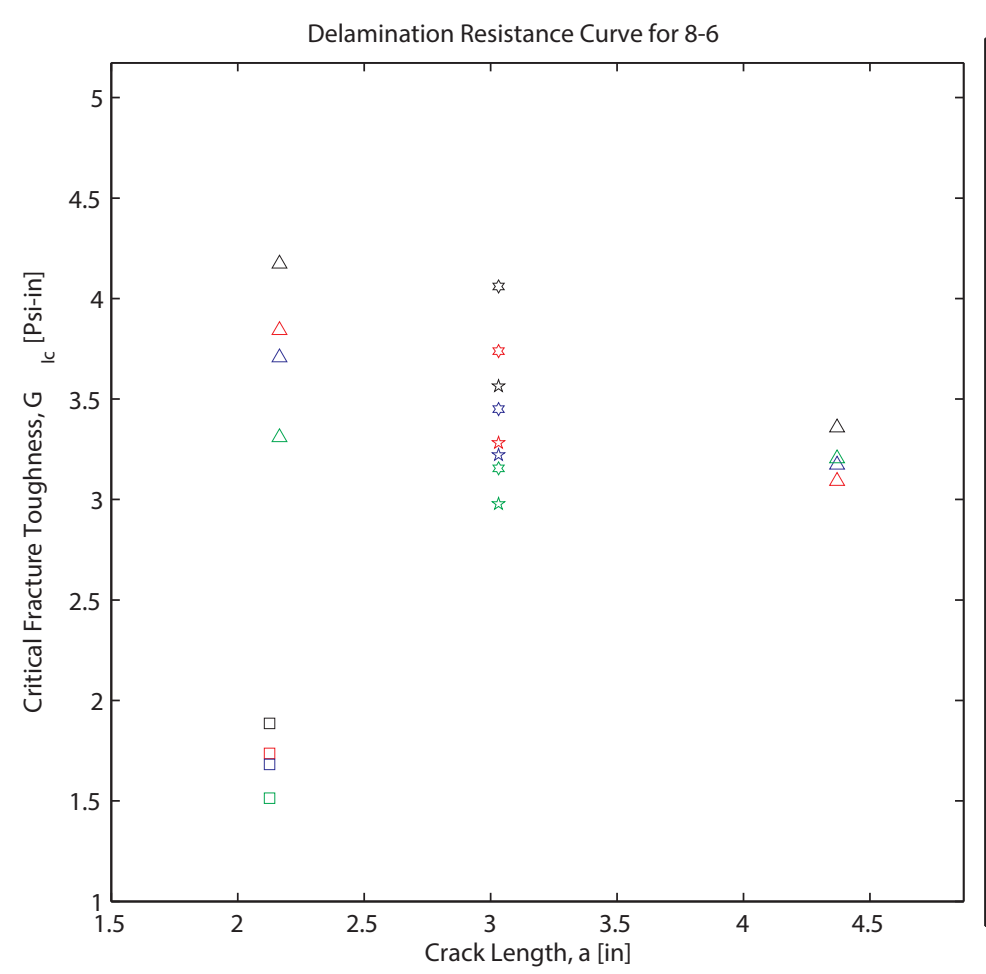

\begin{tabular}{|c|c|}
\hline$\square$ & Visual Onset from Insert -- MBT \\
\hline$\square$ & $\mathrm{CC}$ \\
\hline$\square$ & MCC \\
\hline$\square$ & EQS \\
\hline O & Deviation from Linearity at Insert -- MBT \\
\hline o & $\mathrm{CC}$ \\
\hline o & MCC \\
\hline o & EQS \\
\hline$\diamond$ & $5 \%$ Offset from Insert -- MBT \\
\hline$\diamond$ & $\mathrm{CC}$ \\
\hline$\diamond$ & MCC \\
\hline$\diamond$ & EQS \\
\hline s & Visual Onset from Precrack -- MBT \\
\hline$\Leftrightarrow$ & $\mathrm{CC}$ \\
\hline 级 & MCC \\
\hline s & EQS \\
\hline it & Deviation from Linearity at Precrack -- MBT \\
\hline 解 & $\mathrm{CC}$ \\
\hline 论 & MCC \\
\hline 实 & EQS \\
\hline$x$ & $5 \%$ offset from Precrack-- MBT \\
\hline$\times$ & $\mathrm{CC}$ \\
\hline$x$ & MCC \\
\hline$x$ & EQS \\
\hline$\triangle$ & Crack Propagation -- MBT \\
\hline$\triangle$ & $\mathrm{CC}$ \\
\hline$\triangle$ & MCC \\
\hline$\triangle$ & EQS \\
\hline
\end{tabular}




\section{G.4 R-Curves for Plate 9}
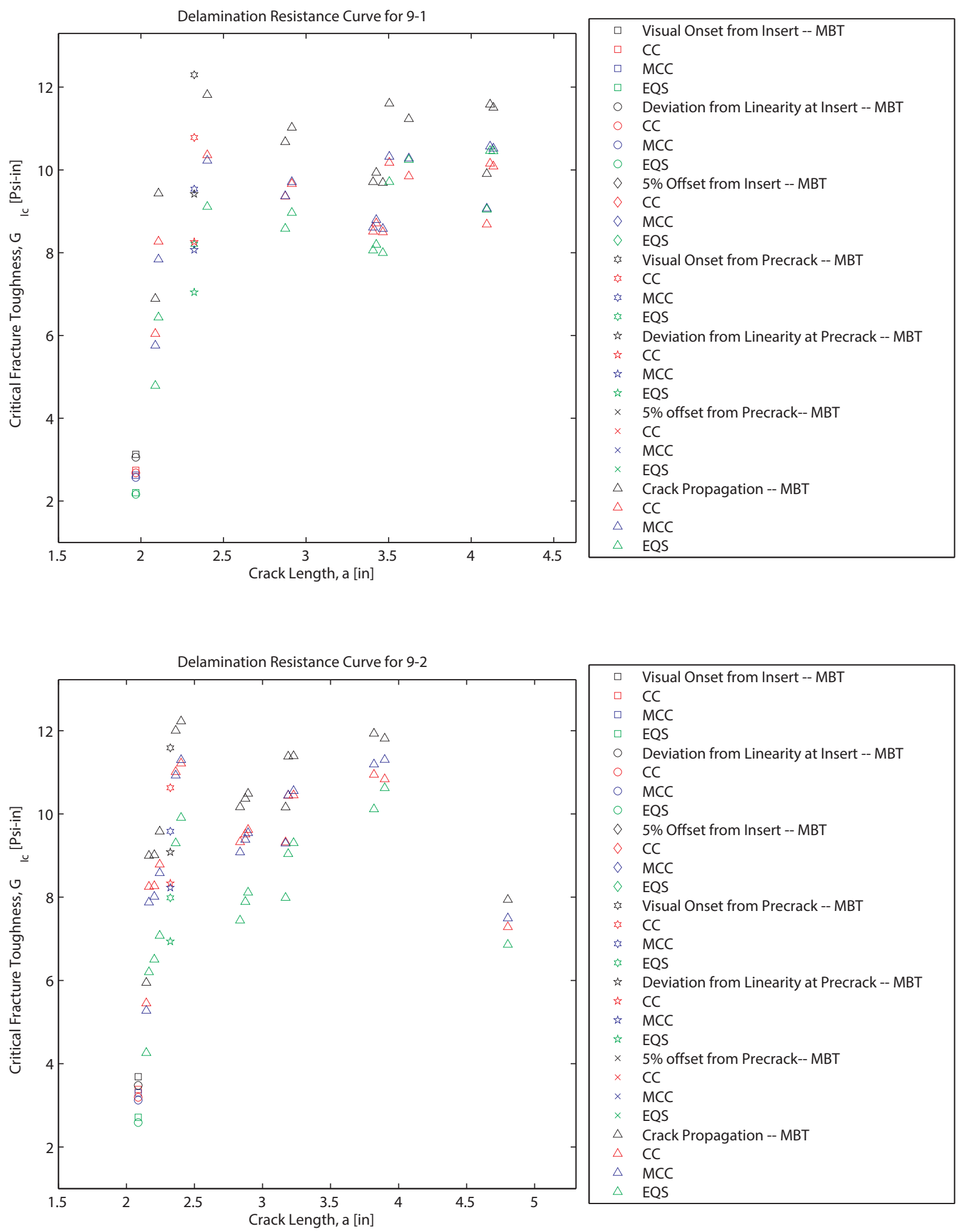

\begin{tabular}{|c|c|}
\hline$\square$ & Visual Onset from Insert -- MBT \\
\hline 口 & $\mathrm{CC}$ \\
\hline 口 & MCC \\
\hline 口 & EQS \\
\hline 0 & Deviation from Linearity at Insert -- MBT \\
\hline 0 & CC \\
\hline 0 & MCC \\
\hline 0 & EQS \\
\hline$\diamond$ & $5 \%$ Offset from Insert -- MBT \\
\hline$\diamond$ & CC \\
\hline$\diamond$ & MCC \\
\hline$\diamond$ & EQS \\
\hline 级 & Visual Onset from Precrack -- MBT \\
\hline 级 & CC \\
\hline 级 & MCC \\
\hline \& & EQS \\
\hline is & Deviation from Linearity at Precrack -- MBT \\
\hline के & CC \\
\hline is & MCC \\
\hline is & EQS \\
\hline$x$ & $5 \%$ offset from Precrack-- MBT \\
\hline$x$ & CC \\
\hline$x$ & MCC \\
\hline$x$ & EQS \\
\hline$\Delta$ & Crack Propagation -- MBT \\
\hline$\Delta$ & $\mathrm{CC}$ \\
\hline$\Delta$ & MCC \\
\hline$\Delta$ & EQS \\
\hline
\end{tabular}




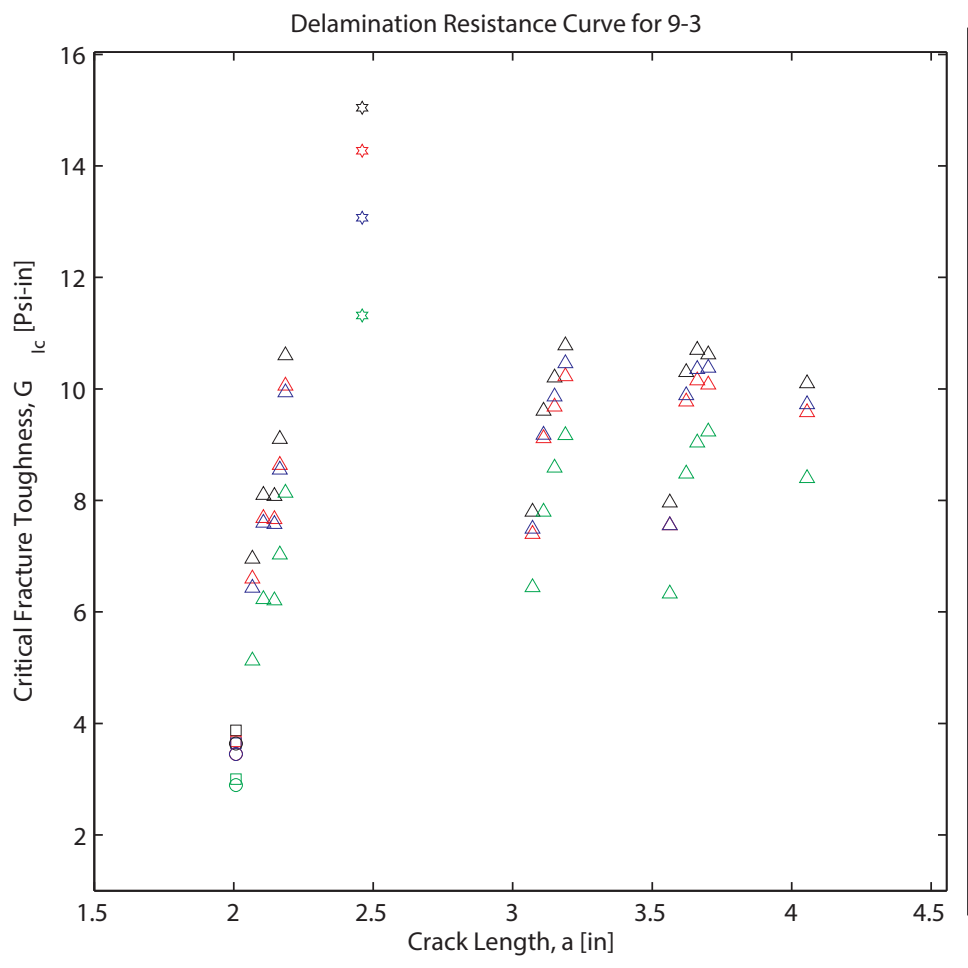

\begin{tabular}{|c|c|}
\hline$\square$ & Visual Onset from Insert -- MBT \\
\hline$\square$ & $\mathrm{CC}$ \\
\hline$\square$ & MCC \\
\hline$\square$ & EQS \\
\hline O & Deviation from Linearity at Insert -- MBT \\
\hline o & $\mathrm{CC}$ \\
\hline o & MCC \\
\hline o & EQS \\
\hline$\diamond$ & $5 \%$ Offset from Insert -- MBT \\
\hline$\diamond$ & $\mathrm{CC}$ \\
\hline$\diamond$ & MCC \\
\hline$\diamond$ & EQS \\
\hline$\Leftrightarrow$ & Visual Onset from Precrack -- MBT \\
\hline 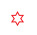 & $\mathrm{CC}$ \\
\hline$\hat{s}$ & MCC \\
\hline$\Leftrightarrow$ & EQS \\
\hline 诂 & Deviation from Linearity at Precrack -- MBT \\
\hline 诂 & $\mathrm{CC}$ \\
\hline is & MCC \\
\hline 它 & EQS \\
\hline$x$ & $5 \%$ offset from Precrack-- MBT \\
\hline$x$ & $\mathrm{CC}$ \\
\hline$x$ & MCC \\
\hline$x$ & EQS \\
\hline$\triangle$ & Crack Propagation -- MBT \\
\hline$\triangle$ & $\mathrm{CC}$ \\
\hline$\triangle$ & MCC \\
\hline$\triangle$ & EQS \\
\hline
\end{tabular}

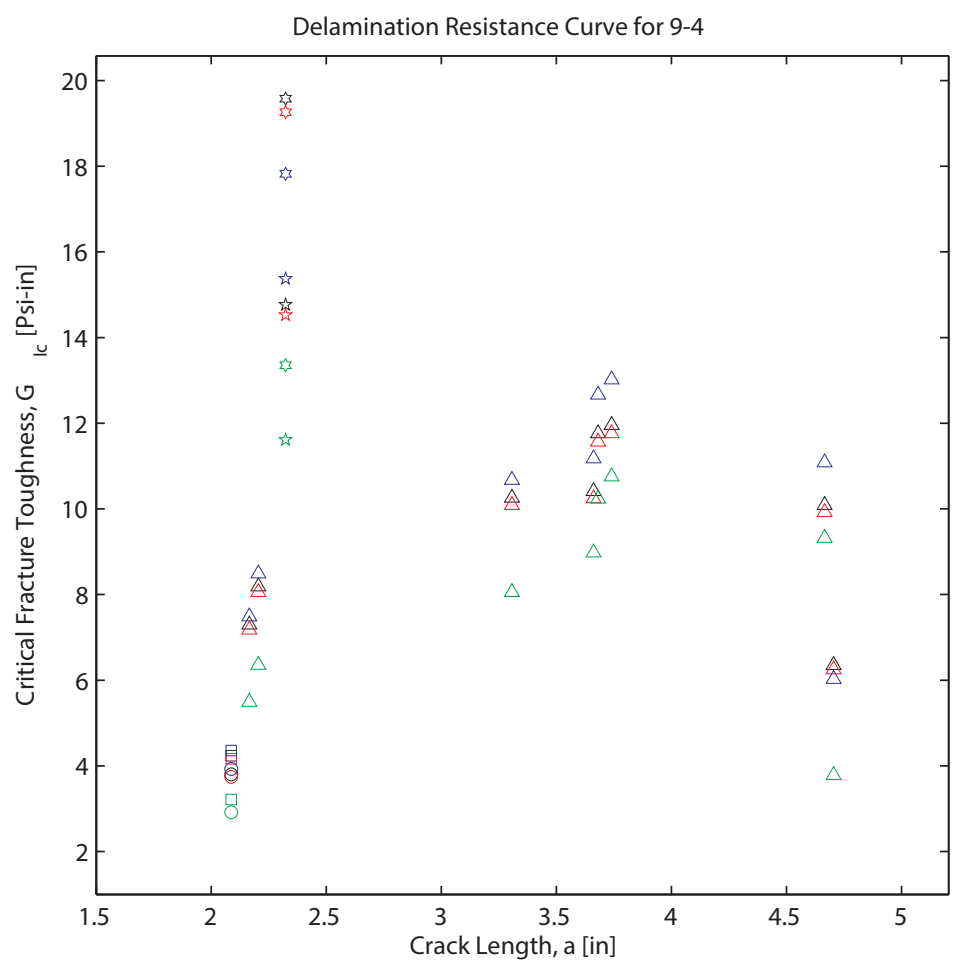

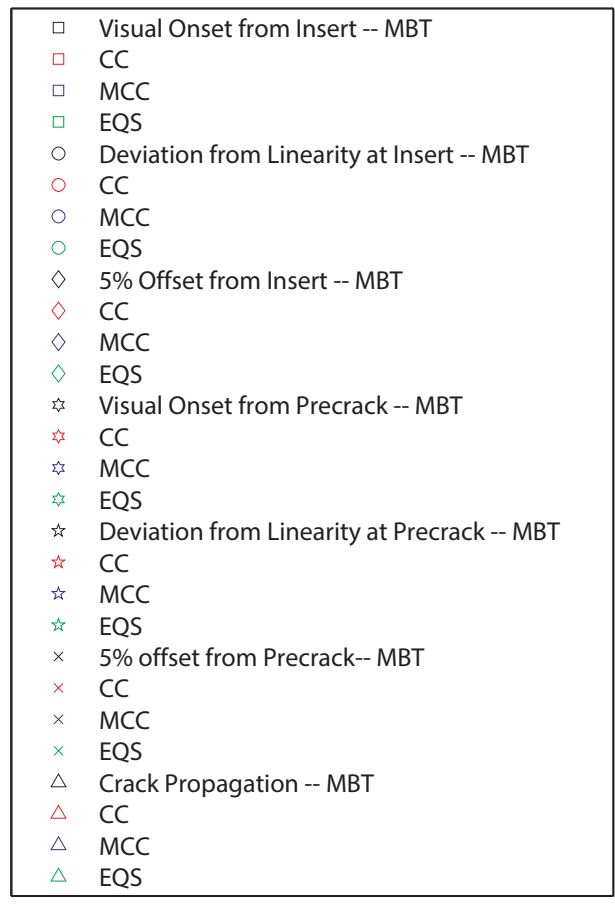




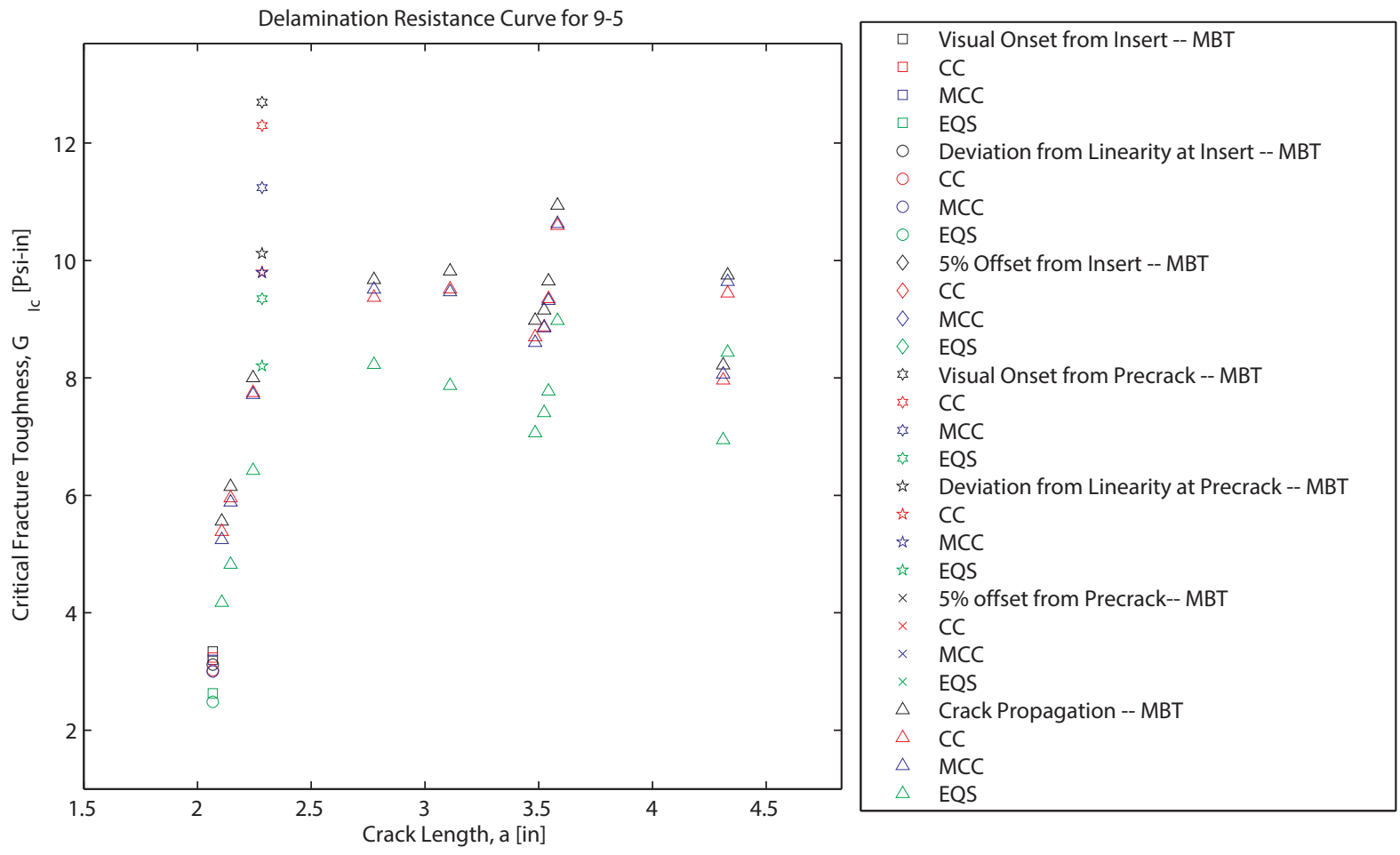




\section{G.5 R-Curves for Plate 10}
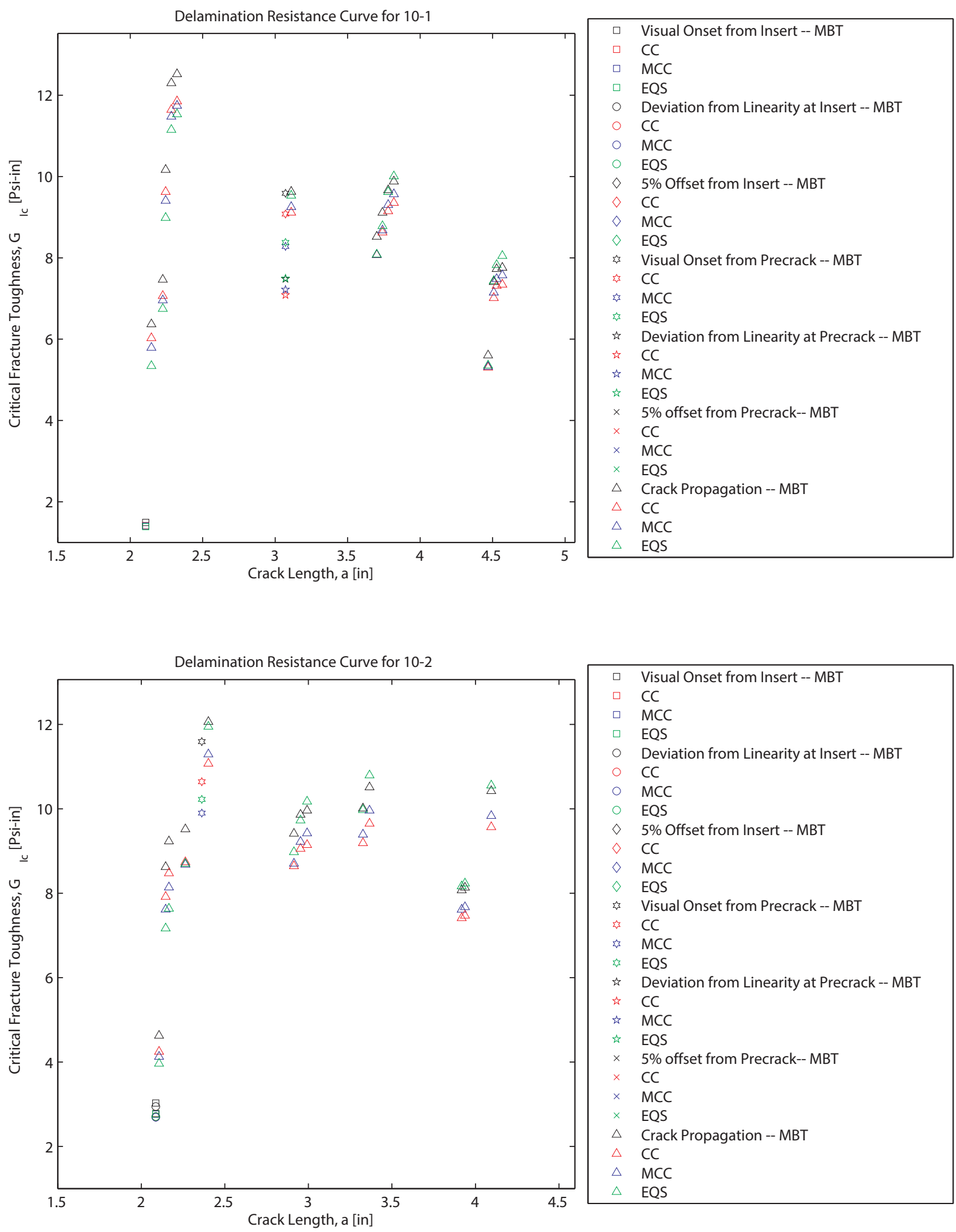

\begin{tabular}{|c|c|}
\hline$\square$ & Visual Onset from Insert -- MBT \\
\hline$\square$ & CC \\
\hline$\square$ & MCC \\
\hline 口 & EQS \\
\hline 0 & Deviation from Linearity at Insert -- MBT \\
\hline 0 & CC \\
\hline 0 & MCC \\
\hline 0 & EQS \\
\hline$\diamond$ & $5 \%$ Offset from Insert -- MBT \\
\hline$\diamond$ & CC \\
\hline$\diamond$ & MCC \\
\hline$\diamond$ & EQS \\
\hline 级 & Visual Onset from Precrack -- MBT \\
\hline 级 & CC \\
\hline is & MCC \\
\hline \& & EQS \\
\hline is & Deviation from Linearity at Precrack -- MBT \\
\hline is & CC \\
\hline is & MCC \\
\hline is & EQS \\
\hline$x$ & $5 \%$ offset from Precrack-- MBT \\
\hline$x$ & CC \\
\hline$x$ & MCC \\
\hline$x$ & EQS \\
\hline$\Delta$ & Crack Propagation -- MBT \\
\hline$\Delta$ & $\mathrm{CC}$ \\
\hline$\triangle$ & MCC \\
\hline$\Delta$ & EQS \\
\hline
\end{tabular}




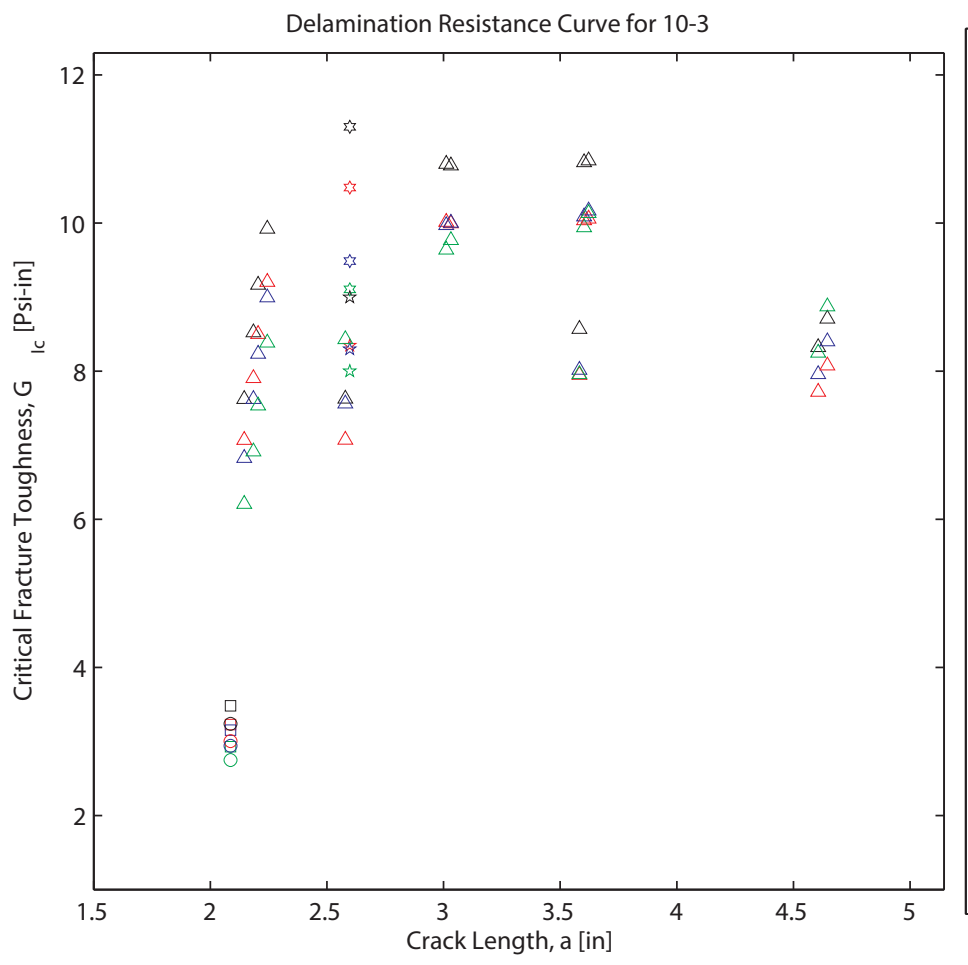

\begin{tabular}{|c|c|}
\hline$\square$ & Visual Onset from Insert -- MBT \\
\hline$\square$ & $\mathrm{CC}$ \\
\hline$\square$ & MCC \\
\hline$\square$ & EQS \\
\hline O & Deviation from Linearity at Insert -- MBT \\
\hline O & $\mathrm{CC}$ \\
\hline O & MCC \\
\hline o & EQS \\
\hline$\diamond$ & $5 \%$ Offset from Insert -- MBT \\
\hline$\diamond$ & $\mathrm{CC}$ \\
\hline$\diamond$ & MCC \\
\hline$\diamond$ & EQS \\
\hline$\Leftrightarrow$ & Visual Onset from Precrack -- MBT \\
\hline 23 & $\mathrm{CC}$ \\
\hline$\sum_{3}$ & MCC \\
\hline 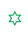 & EQS \\
\hline is & Deviation from Linearity at Precrack -- MBT \\
\hline मे & $\mathrm{CC}$ \\
\hline मे & MCC \\
\hline 放 & EQS \\
\hline$x$ & $5 \%$ offset from Precrack-- MBT \\
\hline$x$ & $\mathrm{CC}$ \\
\hline$x$ & MCC \\
\hline$x$ & EQS \\
\hline$\triangle$ & Crack Propagation -- MBT \\
\hline$\triangle$ & $\mathrm{CC}$ \\
\hline$\triangle$ & MCC \\
\hline$\triangle$ & EQS \\
\hline
\end{tabular}

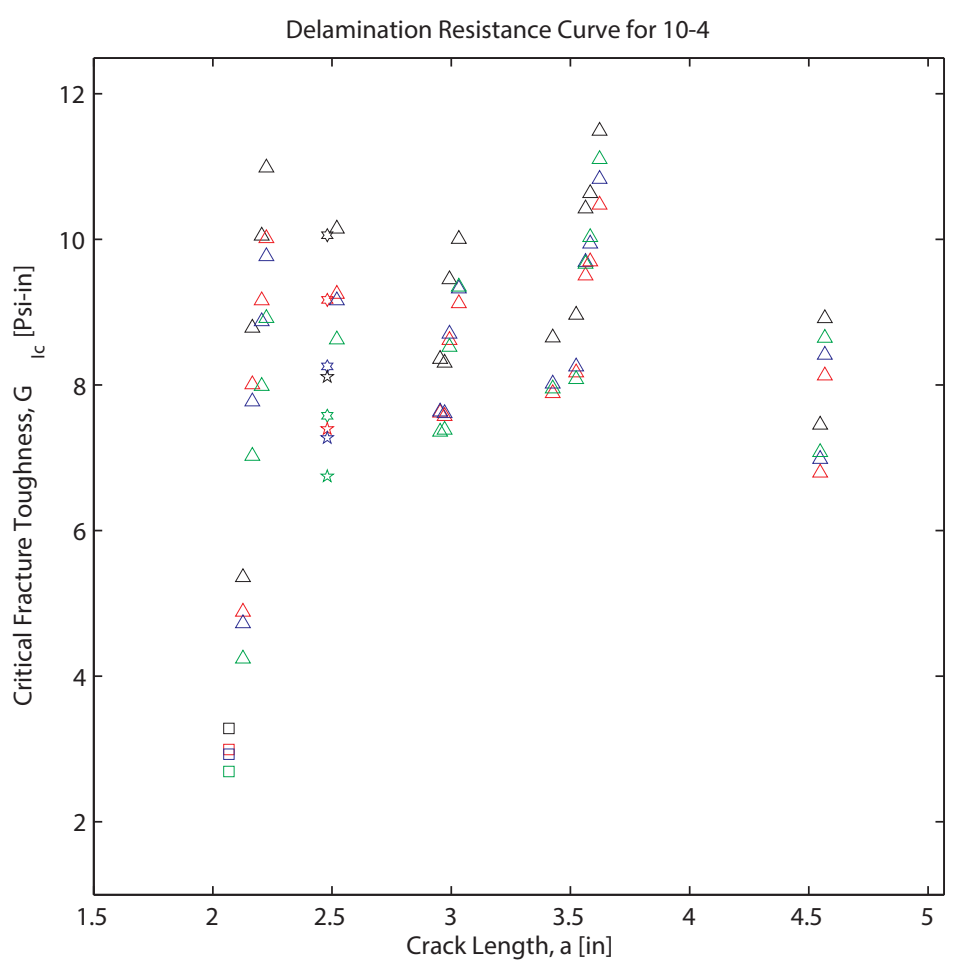

\begin{tabular}{|c|c|}
\hline$\square$ & Visual Onset from Insert -- MBT \\
\hline$\square$ & $\mathrm{CC}$ \\
\hline$\square$ & MCC \\
\hline$\square$ & EQS \\
\hline o & Deviation from Linearity at Insert -- MBT \\
\hline O & $\mathrm{CC}$ \\
\hline o & MCC \\
\hline o & EQS \\
\hline$\diamond$ & $5 \%$ Offset from Insert -- MBT \\
\hline$\diamond$ & $\mathrm{CC}$ \\
\hline$\diamond$ & MCC \\
\hline$\diamond$ & EQS \\
\hline s & Visual Onset from Precrack -- MBT \\
\hline$\sum_{3}$ & $\mathrm{CC}$ \\
\hline s & MCC \\
\hline 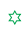 & EQS \\
\hline is & Deviation from Linearity at Precrack -- MBT \\
\hline$\hbar$ & $\mathrm{CC}$ \\
\hline 放 & MCC \\
\hline is & EQS \\
\hline$\times$ & $5 \%$ offset from Precrack-- MBT \\
\hline$\times$ & $\mathrm{CC}$ \\
\hline$x$ & MCC \\
\hline$x$ & EQS \\
\hline$\triangle$ & Crack Propagation -- MBT \\
\hline$\triangle$ & $\mathrm{CC}$ \\
\hline$\triangle$ & MCC \\
\hline$\triangle$ & EQS \\
\hline
\end{tabular}



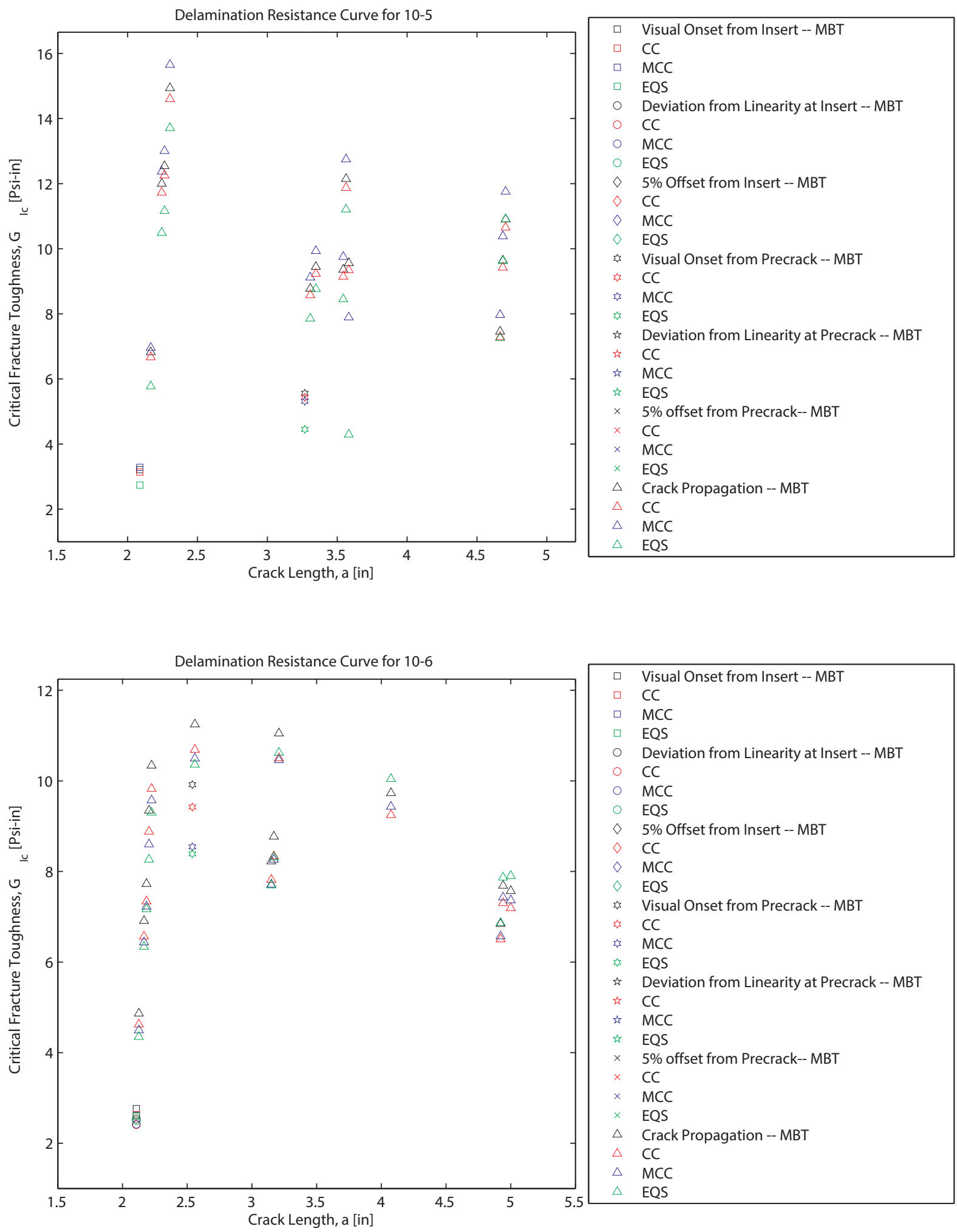


\section{G.6 R-Curves for Plate 11}

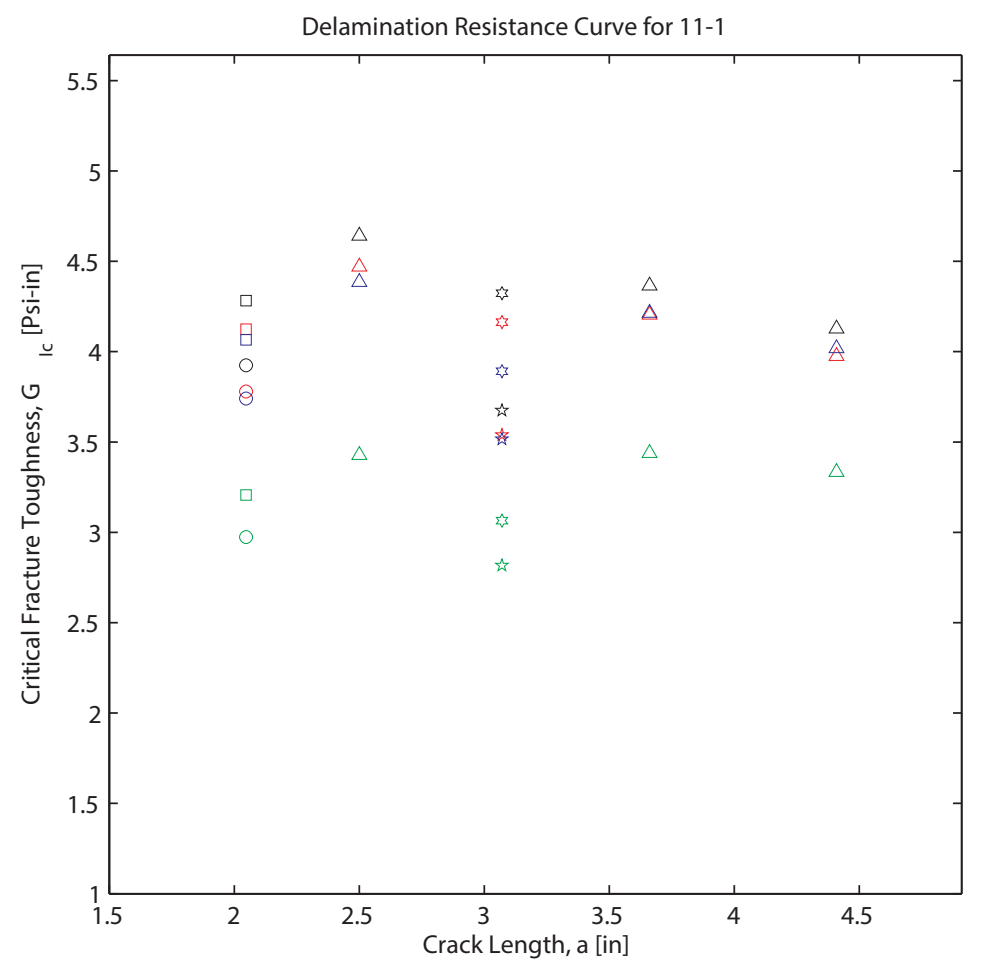

\begin{tabular}{|c|c|}
\hline$\square$ & Visual Onset from Insert -- MBT \\
\hline$\square$ & $\mathrm{CC}$ \\
\hline$\square$ & $\mathrm{MCC}$ \\
\hline$\square$ & EQS \\
\hline O & Deviation from Linearity at Insert -- MBT \\
\hline o & $\mathrm{CC}$ \\
\hline O & MCC \\
\hline O & EQS \\
\hline$\diamond$ & $5 \%$ Offset from Insert -- MBT \\
\hline$\diamond$ & $\mathrm{CC}$ \\
\hline$\diamond$ & MCC \\
\hline$\diamond$ & EQS \\
\hline$\hat{s}$ & Visual Onset from Precrack -- MBT \\
\hline 5 & $\mathrm{CC}$ \\
\hline$\hat{s}$ & MCC \\
\hline$\sqrt{3}$ & EQS \\
\hline$\hat{\omega}$ & Deviation from Linearity at Precrack -- MBT \\
\hline 约 & $\mathrm{CC}$ \\
\hline is & MCC \\
\hline 它 & EQS \\
\hline$x$ & $5 \%$ offset from Precrack-- MBT \\
\hline$x$ & $\mathrm{CC}$ \\
\hline$x$ & MCC \\
\hline$x$ & EQS \\
\hline$\triangle$ & Crack Propagation -- MBT \\
\hline$\triangle$ & $\mathrm{CC}$ \\
\hline$\triangle$ & MCC \\
\hline$\triangle$ & EQS \\
\hline
\end{tabular}

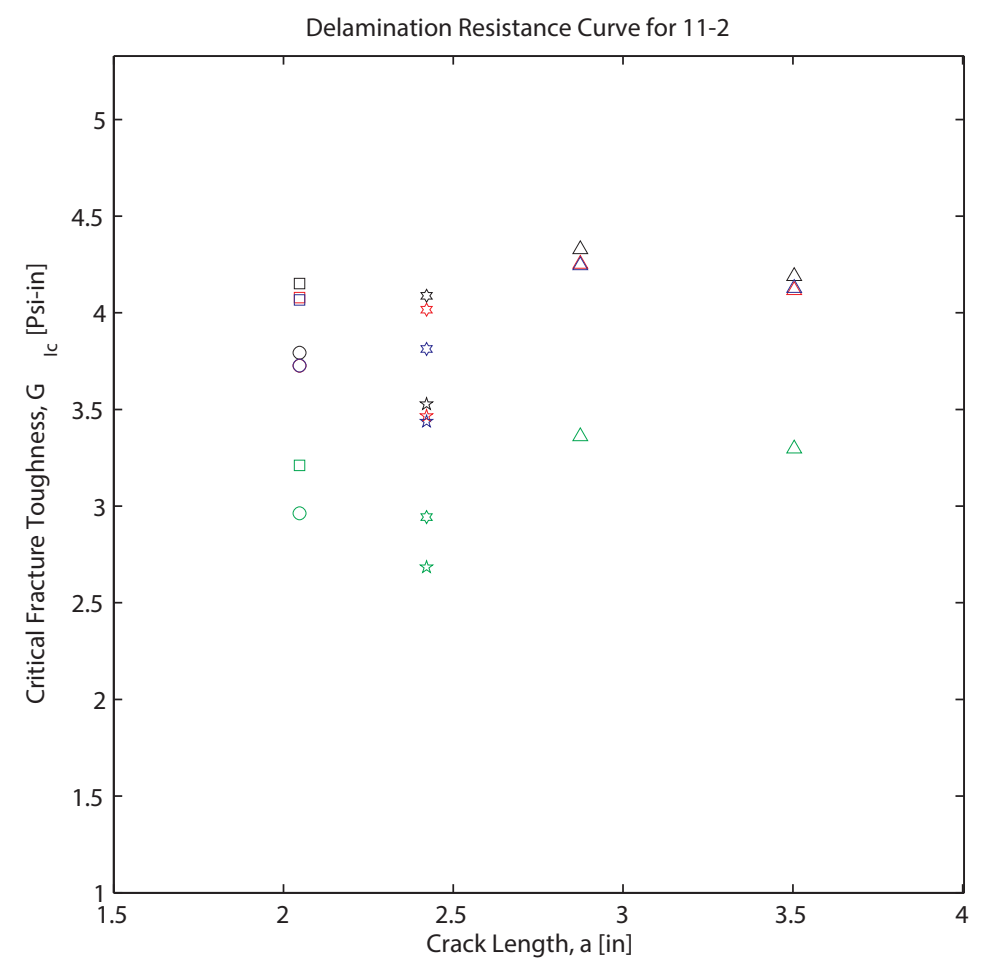

\begin{tabular}{|c|c|}
\hline$\square$ & Visual Onset from Insert -- MBT \\
\hline$\square$ & $\mathrm{CC}$ \\
\hline$\square$ & MCC \\
\hline$\square$ & EQS \\
\hline O & Deviation from Linearity at Insert -- MBT \\
\hline O & $\mathrm{CC}$ \\
\hline O & MCC \\
\hline o & EQS \\
\hline$\diamond$ & $5 \%$ Offset from Insert -- MBT \\
\hline$\diamond$ & $\mathrm{CC}$ \\
\hline$\diamond$ & MCC \\
\hline$\diamond$ & EQS \\
\hline 级 & Visual Onset from Precrack -- MBT \\
\hline$\xi_{3}$ & $\mathrm{CC}$ \\
\hline 急 & MCC \\
\hline 3 & EQS \\
\hline is & Deviation from Linearity at Precrack -- MBT \\
\hline 计 & $\mathrm{CC}$ \\
\hline th & MCC \\
\hline मे & EQS \\
\hline$x$ & $5 \%$ offset from Precrack-- MBT \\
\hline$x$ & $\mathrm{CC}$ \\
\hline$x$ & MCC \\
\hline$x$ & EQS \\
\hline$\triangle$ & Crack Propagation -- MBT \\
\hline$\triangle$ & $\mathrm{CC}$ \\
\hline$\triangle$ & MCC \\
\hline$\triangle$ & EQS \\
\hline
\end{tabular}




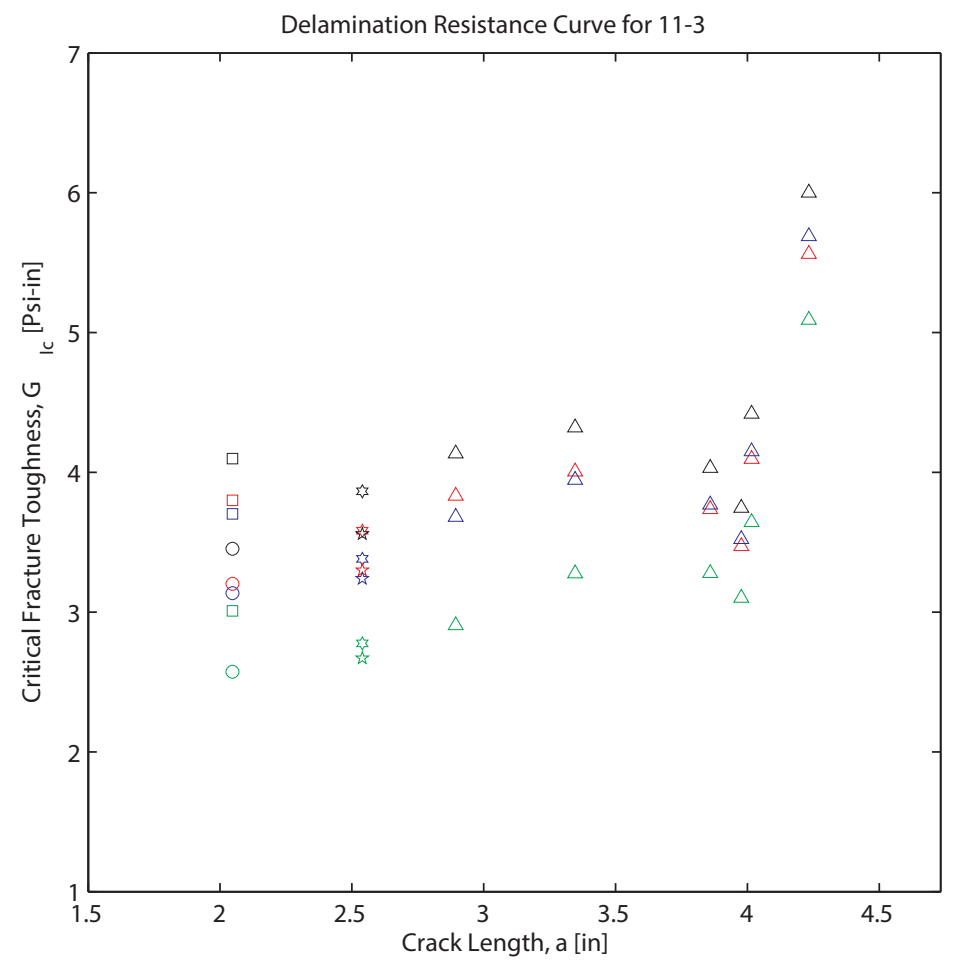

\begin{tabular}{|c|c|}
\hline$\square$ & Visual Onset from Insert -- MBT \\
\hline$\square$ & $\mathrm{CC}$ \\
\hline$\square$ & MCC \\
\hline$\square$ & EQS \\
\hline O & Deviation from Linearity at Insert -- MBT \\
\hline O & $\mathrm{CC}$ \\
\hline o & MCC \\
\hline O & EQS \\
\hline$\diamond$ & $5 \%$ Offset from Insert -- MBT \\
\hline$\diamond$ & $\mathrm{CC}$ \\
\hline$\diamond$ & MCC \\
\hline$\diamond$ & EQS \\
\hline$\Leftrightarrow$ & Visual Onset from Precrack -- MBT \\
\hline 3 & $\mathrm{CC}$ \\
\hline$\hat{s}$ & MCC \\
\hline 3 & EQS \\
\hline is & Deviation from Linearity at Precrack -- MBT \\
\hline 诂 & $\mathrm{CC}$ \\
\hline 放 & MCC \\
\hline is & EQS \\
\hline$x$ & $5 \%$ offset from Precrack-- MBT \\
\hline$x$ & $\mathrm{CC}$ \\
\hline$x$ & MCC \\
\hline$x$ & EQS \\
\hline$\triangle$ & Crack Propagation -- MBT \\
\hline$\triangle$ & $\mathrm{CC}$ \\
\hline$\triangle$ & MCC \\
\hline$\triangle$ & EQS \\
\hline
\end{tabular}

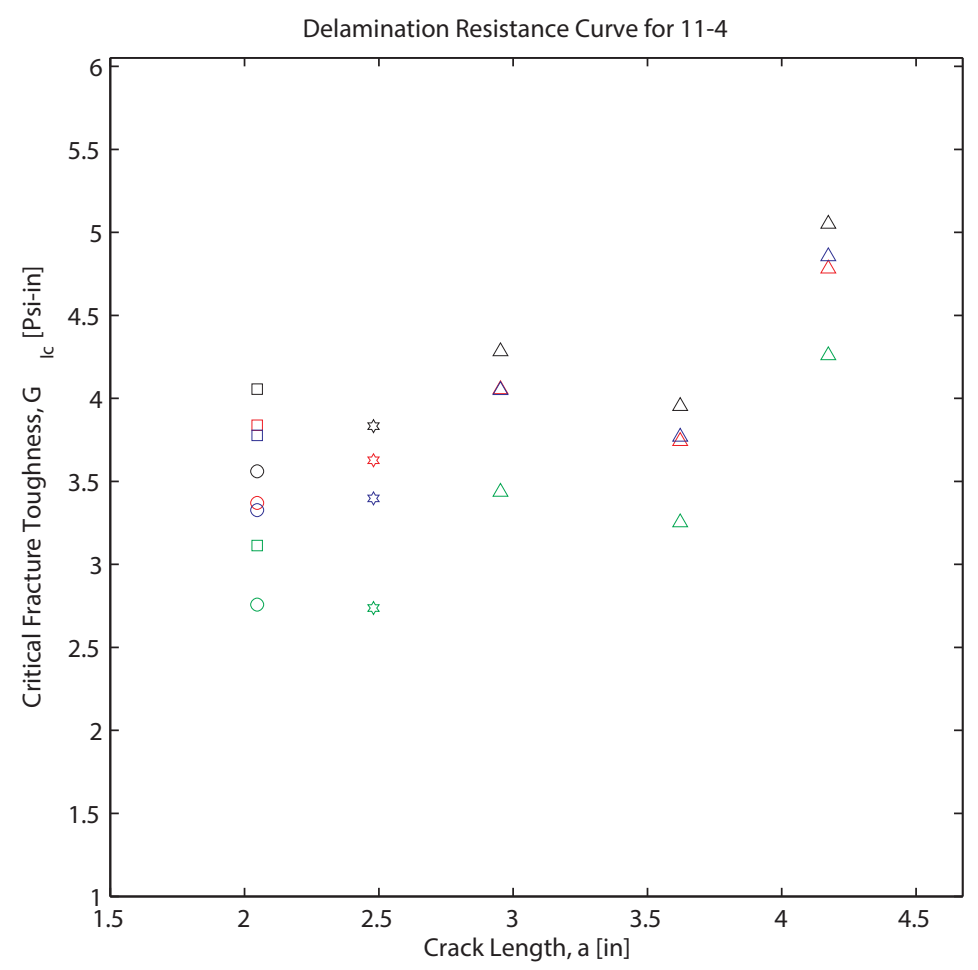

\begin{tabular}{|c|c|}
\hline$\square$ & Visual Onset from Insert -- MBT \\
\hline$\square$ & $\mathrm{CC}$ \\
\hline$\square$ & MCC \\
\hline$\square$ & EQS \\
\hline o & Deviation from Linearity at Insert -- MBT \\
\hline o & $\mathrm{CC}$ \\
\hline o & MCC \\
\hline o & EQS \\
\hline$\diamond$ & $5 \%$ Offset from Insert -- MBT \\
\hline$\diamond$ & $\mathrm{CC}$ \\
\hline$\diamond$ & MCC \\
\hline$\diamond$ & EQS \\
\hline s & Visual Onset from Precrack -- MBT \\
\hline 3 & $\mathrm{CC}$ \\
\hline 皮 & MCC \\
\hline$\xi$ & EQS \\
\hline it & Deviation from Linearity at Precrack -- MBT \\
\hline 解 & $\mathrm{CC}$ \\
\hline is & MCC \\
\hline 诂 & EQS \\
\hline$x$ & $5 \%$ offset from Precrack-- MBT \\
\hline$x$ & $\mathrm{CC}$ \\
\hline$x$ & MCC \\
\hline$x$ & EQS \\
\hline$\triangle$ & Crack Propagation -- MBT \\
\hline$\triangle$ & $\mathrm{CC}$ \\
\hline$\triangle$ & MCC \\
\hline$\triangle$ & EQS \\
\hline
\end{tabular}




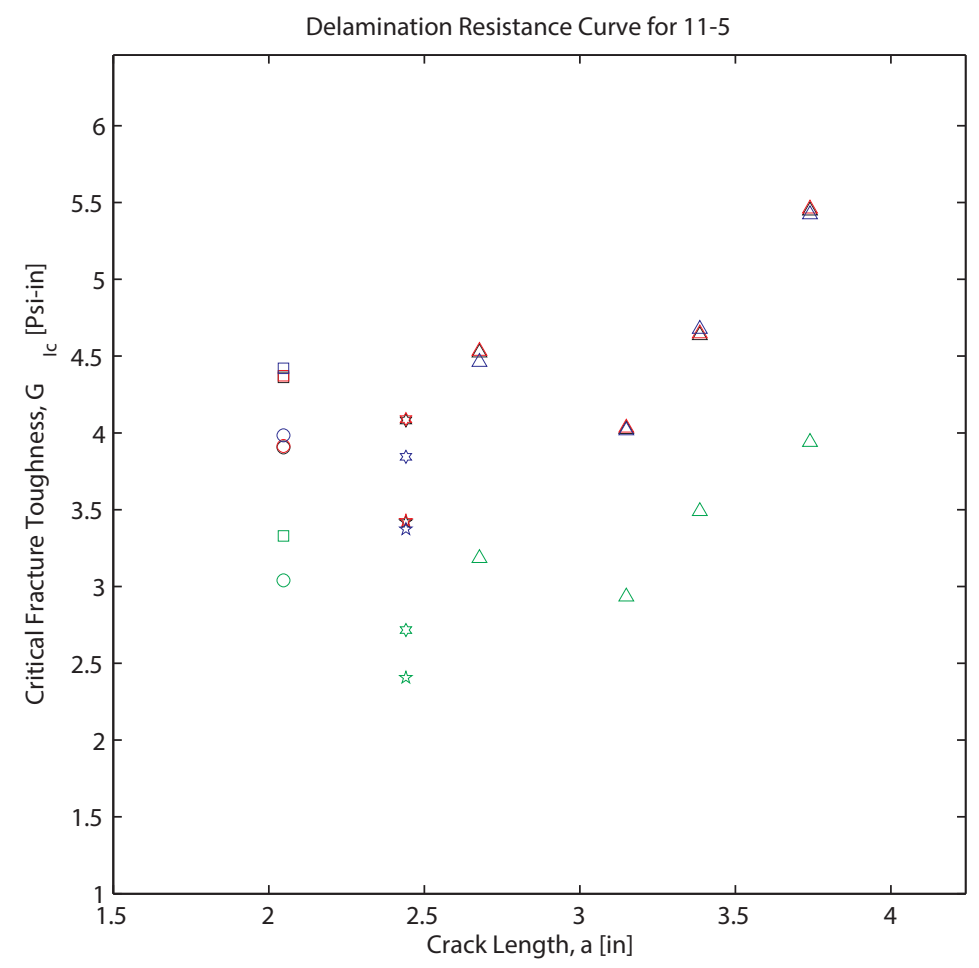

\begin{tabular}{|c|c|}
\hline$\square$ & Visual Onset from Insert -- MBT \\
\hline$\square$ & $\mathrm{CC}$ \\
\hline$\square$ & MCC \\
\hline$\square$ & EQS \\
\hline O & Deviation from Linearity at Insert -- MBT \\
\hline o & $\mathrm{CC}$ \\
\hline O & $\mathrm{MCC}$ \\
\hline O & EQS \\
\hline$\diamond$ & $5 \%$ Offset from Insert -- MBT \\
\hline$\diamond$ & $\mathrm{CC}$ \\
\hline$\diamond$ & MCC \\
\hline$\diamond$ & EQS \\
\hline is & Visual Onset from Precrack -- MBT \\
\hline 级 & $\mathrm{CC}$ \\
\hline is & MCC \\
\hline \& & EQS \\
\hline is & Deviation from Linearity at Precrack -- MBT \\
\hline 虾 & $\mathrm{CC}$ \\
\hline it & MCC \\
\hline is & EQS \\
\hline$x$ & $5 \%$ offset from Precrack-- MBT \\
\hline$x$ & $\mathrm{CC}$ \\
\hline$x$ & MCC \\
\hline$\times$ & EQS \\
\hline$\triangle$ & Crack Propagation -- MBT \\
\hline$\triangle$ & $\mathrm{CC}$ \\
\hline$\triangle$ & MCC \\
\hline$\triangle$ & EQS \\
\hline
\end{tabular}

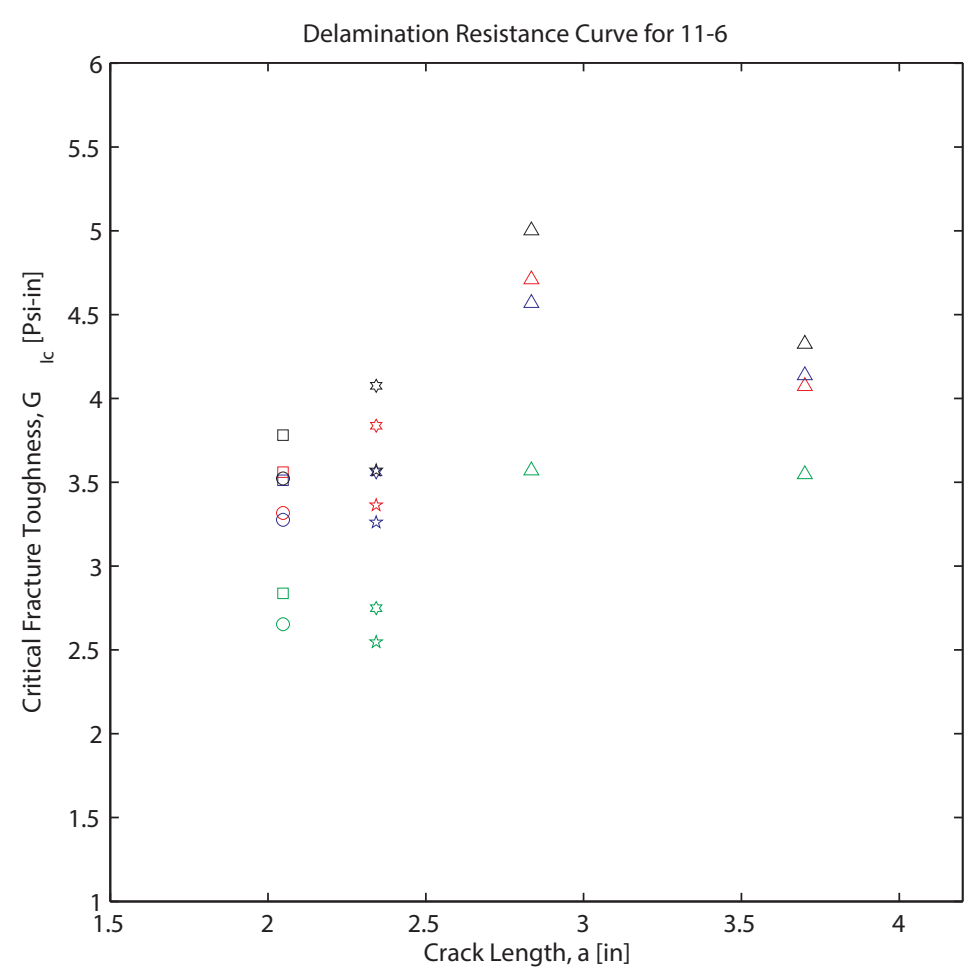

\begin{tabular}{|c|c|}
\hline$\square$ & Visual Onset from Insert -- MBT \\
\hline$\square$ & $\mathrm{CC}$ \\
\hline$\square$ & MCC \\
\hline$\square$ & EQS \\
\hline o & Deviation from Linearity at Insert -- MBT \\
\hline o & $\mathrm{CC}$ \\
\hline o & MCC \\
\hline o & EQS \\
\hline$\diamond$ & $5 \%$ Offset from Insert -- MBT \\
\hline$\diamond$ & $\mathrm{CC}$ \\
\hline$\diamond$ & MCC \\
\hline$\diamond$ & EQS \\
\hline s & Visual Onset from Precrack -- MBT \\
\hline 3 & $\mathrm{CC}$ \\
\hline 急 & MCC \\
\hline$\xi$ & EQS \\
\hline it & Deviation from Linearity at Precrack -- MBT \\
\hline 解 & $\mathrm{CC}$ \\
\hline is & MCC \\
\hline 诂 & EQS \\
\hline$x$ & $5 \%$ offset from Precrack-- MBT \\
\hline$x$ & $\mathrm{CC}$ \\
\hline$x$ & MCC \\
\hline$x$ & EQS \\
\hline$\triangle$ & Crack Propagation -- MBT \\
\hline$\triangle$ & $\mathrm{CC}$ \\
\hline$\triangle$ & MCC \\
\hline$\triangle$ & EQS \\
\hline
\end{tabular}


AppendixH

DCB Data Reports 


\section{H.1 Plate 6 Data Report}

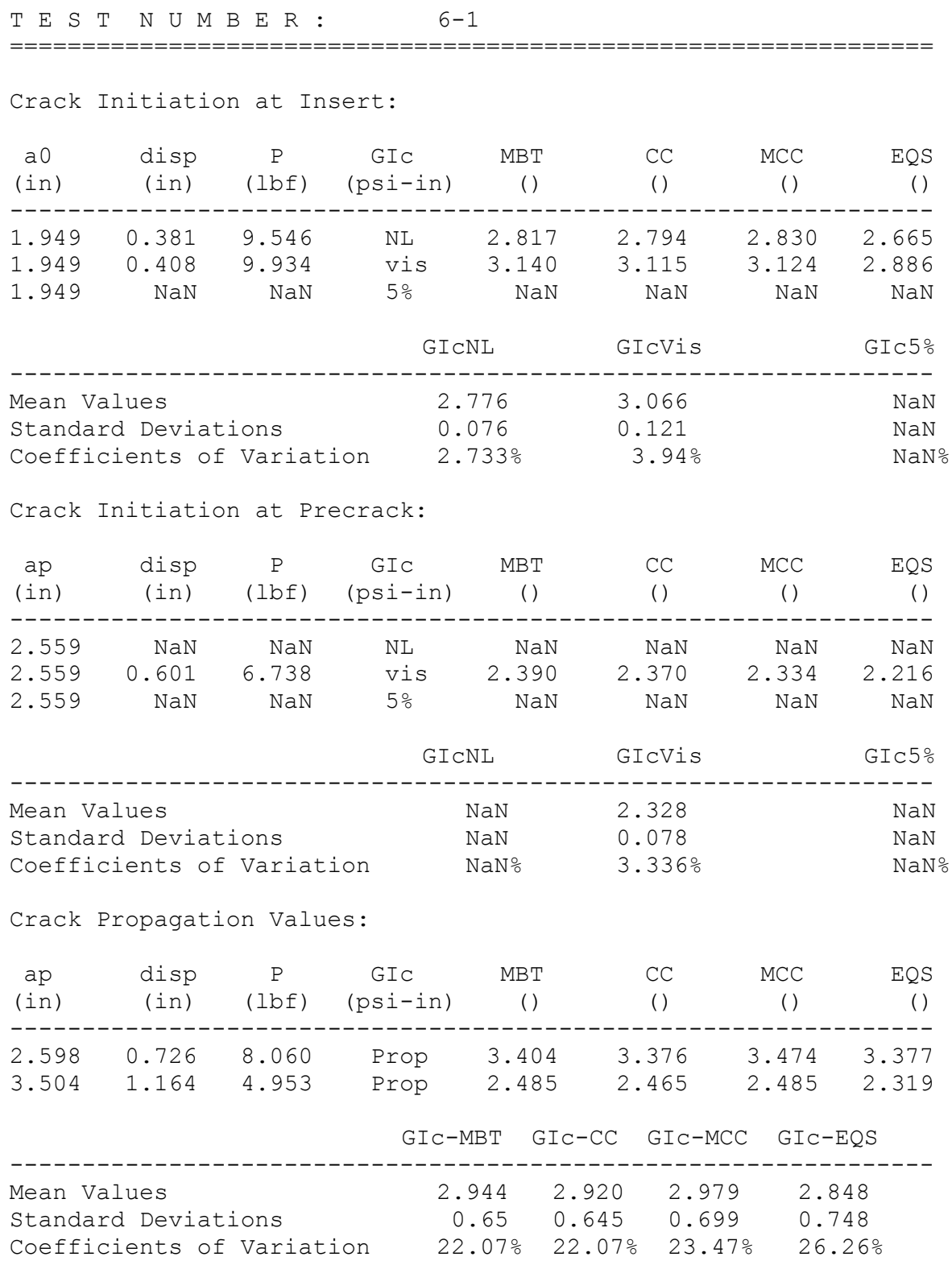


$\begin{array}{llllllllllll}\text { T } & E & S & \mathrm{~T} & \mathrm{~N} & \mathrm{U} & \mathrm{M} & \mathrm{B} & \mathrm{E} & \mathrm{R} & \text { : }\end{array}$

$6-2$

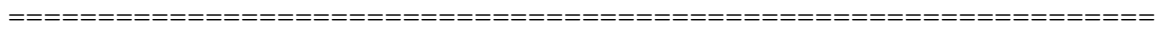

Crack Initiation at Insert:

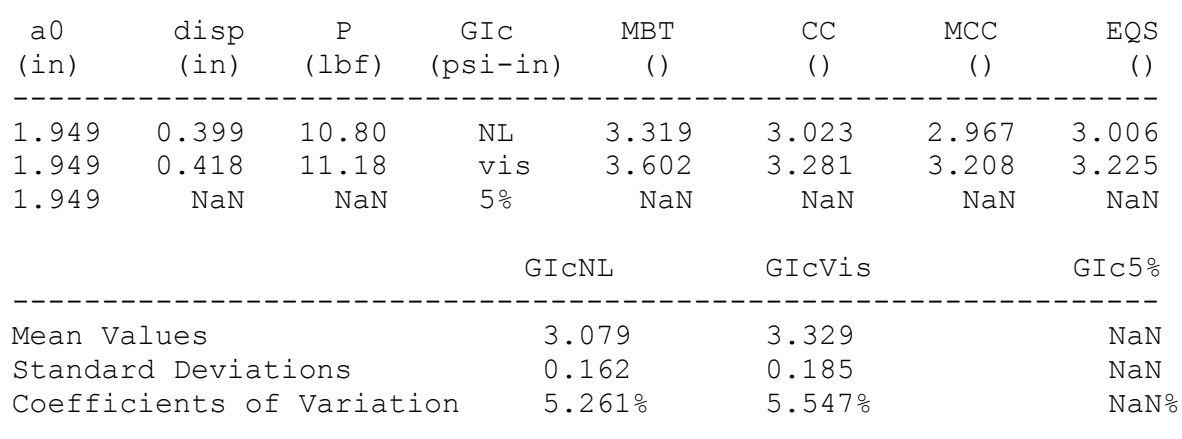

Crack Initiation at Precrack:

\begin{tabular}{|c|c|c|c|c|c|c|c|}
\hline $\begin{array}{l}\operatorname{ap} \\
(i n)\end{array}$ & $\begin{array}{l}\text { disp } \\
(\text { in) }\end{array}$ & $\begin{array}{c}\mathrm{P} \\
(1 \mathrm{~b} f)\end{array}$ & $\begin{array}{c}\text { GIC } \\
(p s i-i n)\end{array}$ & $\begin{array}{r}\mathrm{MBT} \\
()\end{array}$ & $\begin{array}{l}\mathrm{CC} \\
()\end{array}$ & $\begin{array}{c}\mathrm{MCC} \\
()\end{array}$ & $\begin{array}{c}E Q S \\
()\end{array}$ \\
\hline \multicolumn{8}{|c|}{ 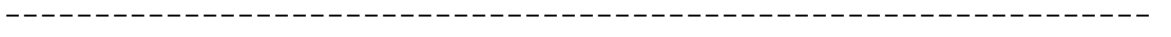 } \\
\hline 2.894 & 0.843 & 7.761 & $\mathrm{NL}$ & 3.396 & 3.093 & 3.146 & 3.424 \\
\hline 2.894 & 0.852 & 7.834 & vis & 3.463 & 3.155 & 3.069 & 3.338 \\
\hline 2.894 & $\mathrm{NaN}$ & $\mathrm{NaN}$ & $5 \%$ & $\mathrm{NaN}$ & NaN & NaN & NaN \\
\hline \multicolumn{5}{|c|}{ GICNL } & GIcVis & & GIC $5 \%$ \\
\hline \multicolumn{5}{|c|}{ Mean Values } & 3.256 & & $\mathrm{NaN}$ \\
\hline \multirow{2}{*}{\multicolumn{3}{|c|}{$\begin{array}{l}\text { Standard Deviations } \\
\text { Coefficients of Variat }\end{array}$}} & & 69 & 0.178 & & $\mathrm{NaN}$ \\
\hline & & & on & $84 \%$ & $5.463 \%$ & & $\mathrm{NaN} \%$ \\
\hline
\end{tabular}

Crack Propagation Values:

\begin{tabular}{|c|c|c|c|c|c|c|c|}
\hline $\begin{array}{l}\mathrm{ap} \\
(\mathrm{in})\end{array}$ & $\begin{array}{l}\text { disp } \\
(i n)\end{array}$ & $\begin{array}{c}\mathrm{P} \\
(1 \mathrm{bf})\end{array}$ & $\begin{array}{c}\text { GIC } \\
(p s i-i n)\end{array}$ & $\begin{array}{c}\mathrm{MBT} \\
(\text { ) }\end{array}$ & $\begin{array}{l}\mathrm{CC} \\
()\end{array}$ & $\begin{array}{c}\mathrm{MCC} \\
(\text { ) }\end{array}$ & $\begin{array}{c}\text { EQS } \\
()\end{array}$ \\
\hline 1.988 & 0.440 & 11.45 & Prop & 3.809 & 3.470 & 3.428 & 3.520 \\
\hline
\end{tabular}

$\begin{array}{lrrrr} & \text { GIC-MBT } & \text { GIC-CC } & \text { GIC-MCC } & \text { GIC-EQS } \\ --------1 & 3.809 & 3.47 & 3.428 & 3.52 \\ \text { Mean Values } & 0 & 0 & 0 & 0 \\ \text { Standard Deviations } & 0 \% & 0 \% & 0 \% & 0 \% \\ \text { Coefficients of Variation } & & 0 \%\end{array}$




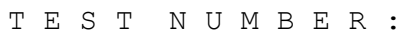

$6-3$

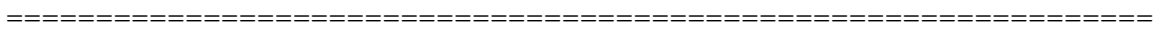

Crack Initiation at Insert:

\begin{tabular}{|c|c|c|c|c|c|c|c|}
\hline $\begin{array}{c}\mathrm{a} 0 \\
(\mathrm{in})\end{array}$ & $\begin{array}{l}\text { disp } \\
(\text { in })\end{array}$ & $\begin{array}{c}\mathrm{P} \\
(\mathrm{lbf})\end{array}$ & $\begin{array}{c}\text { GIC } \\
(p s i-i n)\end{array}$ & $\begin{array}{c}\text { MBT } \\
()\end{array}$ & $\begin{array}{l}\text { CC } \\
()\end{array}$ & $\begin{array}{c}\mathrm{MCC} \\
()\end{array}$ & $\begin{array}{c}\text { EQS } \\
()\end{array}$ \\
\hline \multicolumn{8}{|c|}{ 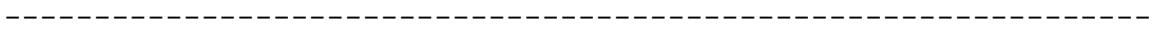 } \\
\hline 1.929 & 0.3396 & 9.424 & NL & 2.428 & 2.353 & 2.344 & 1.664 \\
\hline 1.929 & 0.3512 & 9.717 & vis & 2.589 & 2.509 & 2.497 & 1.769 \\
\hline 1.929 & $\mathrm{NaN}$ & $\mathrm{NaN}$ & $5 \%$ & $\mathrm{NaN}$ & $\mathrm{NaN}$ & $\mathrm{NaN}$ & $\mathrm{NaN}$ \\
\hline \multirow{2}{*}{\multicolumn{7}{|c|}{ GICNL }} & GIC $5 \%$ \\
\hline & & & & 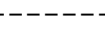 & ------- & & \\
\hline \multicolumn{5}{|c|}{ Mean Values } & \multicolumn{2}{|l|}{2.341} & $\mathrm{NaN}$ \\
\hline \multicolumn{3}{|c|}{ Standard Deviations } & & 58 & \multicolumn{2}{|l|}{0.384} & $\mathrm{NaN}$ \\
\hline \multicolumn{3}{|c|}{ Coefficients of Varj } & & $28 \%$ & \multicolumn{2}{|l|}{$16.39 \%$} & 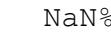 \\
\hline
\end{tabular}

Crack Initiation at Precrack:

\begin{tabular}{|c|c|c|c|c|c|c|c|}
\hline $\begin{array}{l}\text { ap } \\
(i n)\end{array}$ & $\begin{array}{l}\text { disp } \\
\text { (in) }\end{array}$ & $\begin{array}{c}P \\
(1 \mathrm{bf})\end{array}$ & $\begin{array}{c}\text { GIC } \\
(p s i-i n)\end{array}$ & $\begin{array}{c}\mathrm{MBT} \\
(\text { ) }\end{array}$ & $\begin{array}{l}\mathrm{CC} \\
()\end{array}$ & $\begin{array}{c}\mathrm{MCC} \\
()\end{array}$ & \\
\hline 2.323 & 0.5385 & 7.956 & $\mathrm{NL}$ & 2.699 & 2.616 & 2.543 & 1. \\
\hline 2.323 & 0.5496 & 8.102 & vis & 2.806 & 2.719 & 2.550 & 1.7 \\
\hline 2.323 & $\mathrm{NaN}$ & $\mathrm{NaN}$ & $5 \%$ & $\mathrm{NaN}$ & NaN & NaN & \\
\hline
\end{tabular}

\begin{tabular}{|c|c|c|c|}
\hline & GICNL & GICVis & GIC $5 \%$ \\
\hline Mean Values & 2.394 & 2.449 & $\mathrm{NaN}$ \\
\hline Standard Deviations & 0.455 & 0.496 & $\mathrm{NaN}$ \\
\hline Coefficients of Variation & $18.99 \%$ & $20.27 \%$ & $\mathrm{NaN} \%$ \\
\hline
\end{tabular}

Crack Propagation Values:

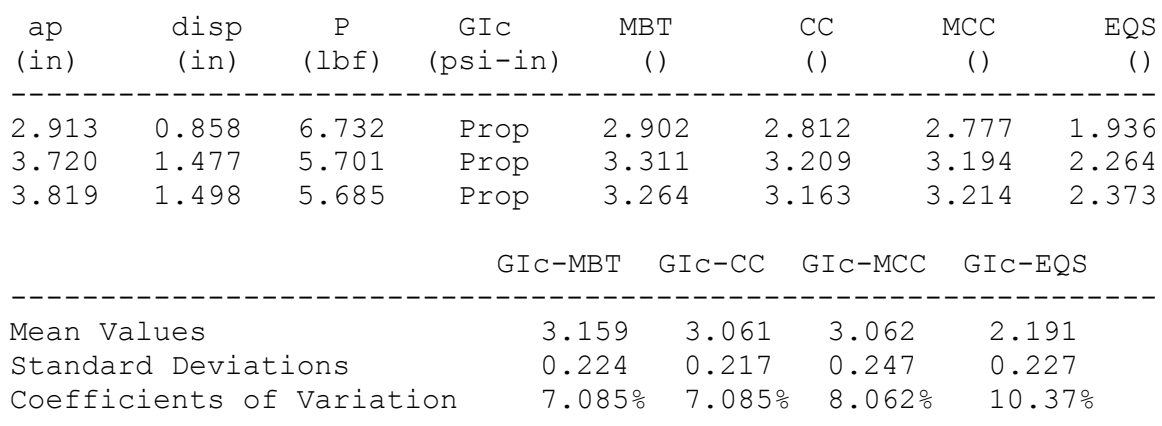


T E S T $\mathrm{S}$ U U M B E R : 6-4

$==\mathrm{=}=\mathrm{=}=\mathrm{=}=\mathrm{=}=\mathrm{=}=\mathrm{=}=\mathrm{=}=\mathrm{=}=\mathrm{=}=\mathrm{=}=\mathrm{=}=\mathrm{=}=\mathrm{=}=\mathrm{=}=\mathrm{=}=\mathrm{=}=\mathrm{=}=\mathrm{=}=\mathrm{=}=\mathrm{=}=\mathrm{=}=\mathrm{=}=\mathrm{=}=\mathrm{=}$

Crack Initiation at Insert:

\begin{tabular}{|c|c|c|c|c|c|c|c|}
\hline $\begin{array}{c}\mathrm{a} 0 \\
(\mathrm{in})\end{array}$ & $\begin{array}{l}\text { disp } \\
(i n)\end{array}$ & $\begin{array}{c}\mathrm{P} \\
(\mathrm{lbf})\end{array}$ & $\begin{array}{c}\text { GIC } \\
(p s i-i n)\end{array}$ & $\begin{array}{c}\text { MBT } \\
()\end{array}$ & $\begin{array}{l}\text { CC } \\
()\end{array}$ & $\begin{array}{c}\mathrm{MCC} \\
()\end{array}$ & $\begin{array}{c}\text { EQS } \\
()\end{array}$ \\
\hline \multicolumn{8}{|c|}{ 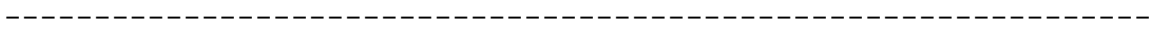 } \\
\hline 1.890 & 0.357 & 10.25 & NL & 2.832 & 2.587 & 2.542 & 1.890 \\
\hline 1.890 & 0.372 & 10.65 & vis & 3.067 & 2.802 & 2.750 & 2.038 \\
\hline 1.890 & $\mathrm{NaN}$ & $\mathrm{NaN}$ & $5 \%$ & $\mathrm{NaN}$ & $\mathrm{NaN}$ & $\mathrm{NaN}$ & $\mathrm{NaN}$ \\
\hline & & & GIC & & & & GIC5\% \\
\hline & & & -1 & --- & ------ & 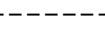 & \\
\hline \multicolumn{5}{|c|}{ Mean Values } & \multicolumn{2}{|l|}{2.664} & \\
\hline \multicolumn{3}{|c|}{ Standard Deviations } & & 02 & \multicolumn{2}{|l|}{0.440} & $\mathrm{NaN}$ \\
\hline \multicolumn{3}{|c|}{ Coefficients of Varia } & & $34 \%$ & \multicolumn{2}{|l|}{$16.52 \%$} & $\mathrm{NaN} \stackrel{\circ}{2}$ \\
\hline
\end{tabular}

Crack Initiation at Precrack:

\begin{tabular}{|c|c|c|c|c|c|c|c|}
\hline $\begin{array}{c}\mathrm{ap} \\
(\mathrm{in})\end{array}$ & $\begin{array}{l}\text { disp } \\
(\text { in) }\end{array}$ & $\begin{array}{c}\mathrm{P} \\
(1 \mathrm{bf})\end{array}$ & $\begin{array}{c}\text { GIC } \\
(p s i-i n)\end{array}$ & $\begin{array}{c}\text { MBT } \\
()\end{array}$ & $\begin{array}{l}\mathrm{CC} \\
()\end{array}$ & $\begin{array}{c}\mathrm{MCC} \\
()\end{array}$ & $\begin{array}{c}\text { EQS } \\
(\text { ) }\end{array}$ \\
\hline 2.579 & 0.546 & 6.882 & NT & 2134 & 1950 & 1985 & 1 \\
\hline 2.579 & 0.568 & 7.101 & vis & 2.288 & 2.090 & 2.061 & 1.638 \\
\hline 2.579 & NaN & $\mathrm{NaN}$ & $5 \%$ & $\mathrm{NaN}$ & $\mathrm{NaN}$ & NaN & $\mathrm{N}$ \\
\hline
\end{tabular}

\begin{tabular}{|c|c|c|c|}
\hline & GICNL & GICVis & GIC $5 \%$ \\
\hline 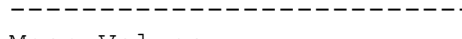 & 1 & מ ח ח & NT \\
\hline Mean Values & 1.914 & 2.020 & $\mathrm{NaN}$ \\
\hline Standard Deviations & 0.233 & 0.273 & $\mathrm{NaN}$ \\
\hline Coefficients of Variation & $12.18 \%$ & $13.53 \%$ & $\mathrm{NaN} \%$ \\
\hline
\end{tabular}

Crack Propagation Values:

\begin{tabular}{|c|c|c|c|c|c|c|c|}
\hline $\begin{array}{l}\mathrm{ap} \\
(\mathrm{in})\end{array}$ & $\begin{array}{l}\text { disp } \\
(i n)\end{array}$ & $\begin{array}{c}P \\
(1 \mathrm{bf})\end{array}$ & $\begin{array}{c}\text { GIC } \\
(\mathrm{psi}-i n)\end{array}$ & $\begin{array}{c}\text { MBT } \\
()\end{array}$ & $\begin{array}{l}\mathrm{CC} \\
()\end{array}$ & $\begin{array}{c}\mathrm{MCC} \\
()\end{array}$ & $\begin{array}{c}\text { EQS } \\
()\end{array}$ \\
\hline 3.051 & 0.851 & 6.516 & Prop & 2.659 & 2.429 & 2.480 & 1.989 \\
\hline
\end{tabular}

$\begin{array}{lrrrr} & \text { GIC-MBT } & \text { GIC-CC } & \text { GIC-MCC } & \text { GIC-EQS } \\ --------------------------------------------- \\ \text { Mean Values } & 2.659 & 2.429 & 2.480 & 1.989 \\ \text { Standard Deviations } & 0 & 0 & 0 & 0 \\ \text { Coefficients of Variation } & 0 \% & 0 \% & 0 \% & 0 \%\end{array}$




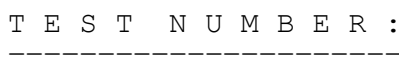

$6-5$

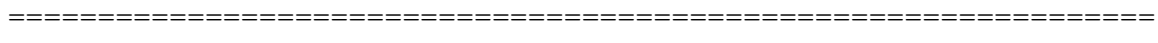

Crack Initiation at Insert:

\begin{tabular}{|c|c|c|c|c|c|c|c|}
\hline $\begin{array}{c}\mathrm{a} 0 \\
(\mathrm{in})\end{array}$ & $\begin{array}{l}\text { disp } \\
(\text { in) }\end{array}$ & $\begin{array}{c}P \\
(1 b f)\end{array}$ & $\begin{array}{c}\text { GIC } \\
(p s i-i n)\end{array}$ & $\begin{array}{r}\mathrm{MBT} \\
()\end{array}$ & $\begin{array}{l}\text { CC } \\
()\end{array}$ & $\begin{array}{c}\mathrm{MCC} \\
()\end{array}$ & $\begin{array}{c}\text { EQS } \\
()\end{array}$ \\
\hline 1.909 & $\mathrm{NaN}$ & $\mathrm{NaN}$ & $\begin{array}{c}\mathrm{NL} \\
\mathrm{NL}\end{array}$ & $\mathrm{NaN}$ & $\mathrm{NaN}$ & $\mathrm{NaN}$ & $\mathrm{NaN}$ \\
\hline 1.909 & 0.291 & 8.252 & vis & 1.828 & 1.788 & 1.781 & 1.172 \\
\hline 1.909 & $\mathrm{NaN}$ & $\mathrm{NaN}$ & $5 \%$ & $\mathrm{NaN}$ & $\mathrm{NaN}$ & $\mathrm{NaN}$ & NaN \\
\hline \multirow{2}{*}{\multicolumn{7}{|c|}{$\begin{array}{l}\mathrm{GICNL} \\
-------\end{array}$}} & GIC $5 \%$ \\
\hline----- & & -- & ------ & ------ & ------- & ------ & ------ \\
\hline \multicolumn{7}{|c|}{ Mean Values } & $\mathrm{NaN}$ \\
\hline \multirow{2}{*}{\multicolumn{4}{|c|}{ Standard Deviations }} & $\mathrm{NaN}$ & 0.314 & & $\mathrm{NaN}$ \\
\hline & & & & $\mathrm{NaN} \%$ & $19.12 \%$ & & $\mathrm{NaN} \circ$ \\
\hline
\end{tabular}

Crack Initiation at Precrack:

\begin{tabular}{|c|c|c|c|c|c|c|c|}
\hline $\begin{array}{l}\text { ap } \\
(i n)\end{array}$ & $\begin{array}{l}\text { disp } \\
(\text { in) }\end{array}$ & $\begin{array}{c}\mathrm{P} \\
(1 \mathrm{bf})\end{array}$ & $\begin{array}{c}\text { GIC } \\
(p s i-i n)\end{array}$ & $\begin{array}{c}\mathrm{MBT} \\
()\end{array}$ & $\begin{array}{l}\mathrm{CC} \\
()\end{array}$ & $\begin{array}{c}\mathrm{MCC} \\
()\end{array}$ & $\begin{array}{c}\text { EQS } \\
()\end{array}$ \\
\hline \multicolumn{8}{|c|}{ 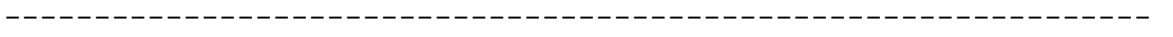 } \\
\hline 2.146 & 0.414 & 8.2 & NL & 2.299 & 2.249 & 2.233 & 1.462 \\
\hline 2.146 & 0.434 & 8.545 & vis & 2.512 & 2.456 & 2.365 & 1.542 \\
\hline 2.146 & $\mathrm{NaN}$ & $\mathrm{NaN}$ & $5 \%$ & $\mathrm{NaN}$ & $\mathrm{NaN}$ & $\mathrm{NaN}$ & $\mathrm{NaN}$ \\
\hline \multicolumn{7}{|c|}{ GICNL } & GIC $5 \%$ \\
\hline & & & 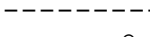 & & ------ & & 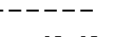 \\
\hline \multicolumn{5}{|c|}{ Mean Values } & \multicolumn{2}{|l|}{2.219} & $\mathrm{NaN}$ \\
\hline \multicolumn{3}{|c|}{ Standard Deviations } & & 00 & \multicolumn{2}{|l|}{0.455} & $\mathrm{NaN}$ \\
\hline \multicolumn{3}{|c|}{ Coefficients of Variat } & on & $42 \%$ & \multicolumn{2}{|l|}{$20.52 \%$} & $\mathrm{NaN} \%$ \\
\hline
\end{tabular}

Crack Propagation Values:

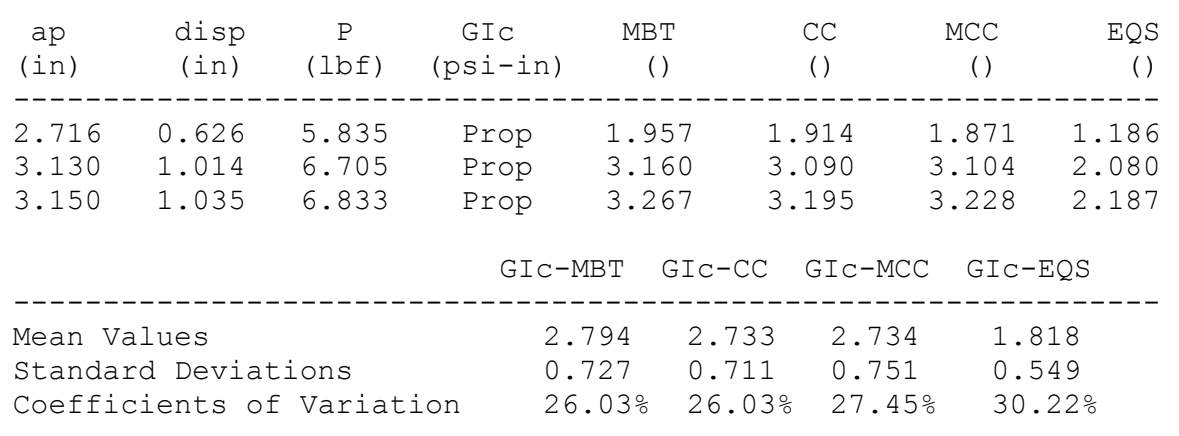


T E S T $\mathrm{S} \quad \mathrm{N}$ U M B E R : 6-6

$==\mathrm{=}=\mathrm{=}=\mathrm{=}=\mathrm{=}=\mathrm{=}=\mathrm{=}=\mathrm{=}=\mathrm{=}=\mathrm{=}=\mathrm{=}=\mathrm{=}=\mathrm{=}=\mathrm{=}=\mathrm{=}=\mathrm{=}=\mathrm{=}=\mathrm{=}=\mathrm{=}=\mathrm{=}=\mathrm{=}=\mathrm{=}=\mathrm{=}=\mathrm{=}=\mathrm{=}$

Crack Initiation at Insert:

\begin{tabular}{|c|c|c|c|c|c|c|c|}
\hline $\begin{array}{c}\mathrm{a} 0 \\
(\mathrm{in})\end{array}$ & $\begin{array}{l}\text { disp } \\
(\text { in) }\end{array}$ & $\begin{array}{c}P \\
(1 \mathrm{~b} f)\end{array}$ & $\begin{array}{c}\text { GIC } \\
(p s i-i n)\end{array}$ & $\begin{array}{c}\mathrm{MBT} \\
(\text { ) }\end{array}$ & $\begin{array}{l}\mathrm{CC} \\
()\end{array}$ & $\begin{array}{c}\mathrm{MCC} \\
()\end{array}$ & \\
\hline 09 & 0.389 & 9.232 & $\mathrm{NL}$ & 2.734 & 2.683 & 2.705 & \\
\hline 09 & 0.408 & 9.329 & vis & 2.902 & 2.848 & 2.834 & \\
\hline 909 & NaN & $\mathrm{NaN}$ & $5 \%$ & $\mathrm{NaN}$ & $\mathrm{NaN}$ & $\mathrm{NaN}$ & \\
\hline
\end{tabular}

1.909 $\quad \mathrm{NaN} \quad \mathrm{NaN}$ \%

GICNL GICVis GIC5응

\begin{tabular}{|c|c|c|c|}
\hline Mean Values & 2.397 & 2.521 & $\mathrm{NaN}$ \\
\hline Standard Deviations & 0.620 & 0.682 & $\mathrm{NaN}$ \\
\hline Coefficients of Variation & $25.88 \%$ & $27.06 \%$ & $\mathrm{NaN} \%$ \\
\hline
\end{tabular}

Crack Initiation at Precrack:

\begin{tabular}{|c|c|c|c|c|c|c|c|}
\hline $\begin{array}{l}\mathrm{ap} \\
(\mathrm{in})\end{array}$ & $\begin{array}{l}\text { disp } \\
(i n)\end{array}$ & $\begin{array}{c}\mathrm{P} \\
(1 \mathrm{bf})\end{array}$ & $\begin{array}{c}\text { GIC } \\
(p s i-i n)\end{array}$ & $\begin{array}{c}\mathrm{MBT} \\
()\end{array}$ & $\begin{array}{l}\mathrm{CC} \\
()\end{array}$ & $\begin{array}{c}\mathrm{MCC} \\
()\end{array}$ & \\
\hline & & & & --- & ------ & ----- & \\
\hline .461 & 0.680 & 8.127 & NL & 3.269 & 3.208 & 3.314 & \\
\hline 2.461 & 0.701 & 8.347 & vis & 3.458 & 3.393 & 3.347 & \\
\hline 2.461 & $\mathrm{NaN}$ & NaN & $5 \%$ & NaN & $\mathrm{NaN}$ & NaN & \\
\hline
\end{tabular}

\begin{tabular}{|c|c|c|c|}
\hline & GICNL & GICVis & GIC $5 \%$ \\
\hline Mean Values & 2920 & 3025 & $\mathrm{~N}$ \\
\hline Standard Deviations & 0.689 & 0.749 & NaN \\
\hline Coefficients of Variation & $23.6 \%$ & $24.77 \%$ & \\
\hline
\end{tabular}

Crack Propagation Values:

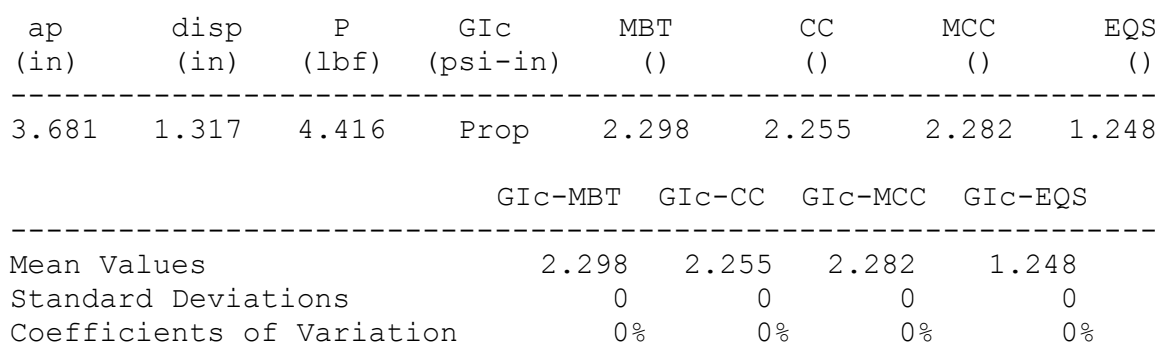


P L A T E

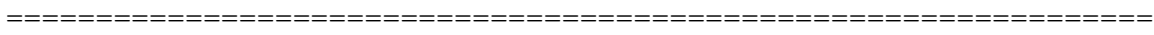

Crack Initiation at Insert

\begin{tabular}{|c|c|c|c|c|c|c|c|}
\hline $\begin{array}{c}\mathrm{a} 0 \\
(\mathrm{in})\end{array}$ & $\begin{array}{l}\text { disp } \\
\text { (in) }\end{array}$ & $\begin{array}{c}\mathrm{P} \\
(1 \mathrm{bf})\end{array}$ & $\begin{array}{c}\text { GIC } \\
(p s i-i n)\end{array}$ & $\begin{array}{c}\mathrm{MBT} \\
()\end{array}$ & $\begin{array}{l}\mathrm{CC} \\
()\end{array}$ & $\begin{array}{c}\mathrm{MCC} \\
()\end{array}$ & $\begin{array}{c}E Q S \\
(\text { ) }\end{array}$ \\
\hline \multicolumn{8}{|c|}{ Mean Values------------------------------------------------------- } \\
\hline 1.923 & 0.373 & 9.850 & NL & 2.826 & 2.688 & 2.677 & 2.138 \\
\hline 1.923 & 0.375 & 9.844 & vis & 2.855 & 2.724 & 2.699 & 2.098 \\
\hline 1.923 & $\mathrm{NaN}$ & $\mathrm{NaN}$ & $5 \%$ & NaN & $\mathrm{NaN}$ & $\mathrm{NaN}$ & $\mathrm{NaN}$ \\
\hline \multicolumn{8}{|c|}{ Standard Deviations---------------------------------------------} \\
\hline $2.38 e-02$ & 0.024 & 0.655 & $\mathrm{NL}$ & 0.320 & 0.248 & 0.244 & 0.665 \\
\hline $8 e-02$ & 0.048 & 1.030 & vis & 0.602 & 0.530 & 0.519 & 0.802 \\
\hline $2.38 e-02$ & 0.024 & NaN & $5 \%$ & $\mathrm{NaN}$ & $\mathrm{NaN}$ & $\mathrm{NaN}$ & $\mathrm{NaN}$ \\
\hline \multicolumn{8}{|c|}{ Coefficients of Variation $\left(\frac{\circ}{\circ}\right)-------------------------------------$} \\
\hline $1.24 e+00$ & 6.522 & 6.651 & NL & 11.34 & 9.236 & 9.100 & 31.08 \\
\hline $4 e+00$ & 12.91 & 10.43 & vis & 21.08 & 19.47 & 19.21 & 38.25 \\
\hline $4 e+00$ & $\mathrm{NaN}$ & NaN & $5 \%$ & NaN & $\mathrm{NaN}$ & $\mathrm{NaN}$ & $\mathrm{NaN}$ \\
\hline
\end{tabular}

Crack Initiation at Precrack

\begin{tabular}{|c|c|c|c|c|c|c|c|}
\hline $\begin{array}{l}\operatorname{ap} \\
(i n)\end{array}$ & $\begin{array}{c}\text { disp } \\
(i n)\end{array}$ & $\begin{array}{c}\mathrm{P} \\
(1 \mathrm{bf})\end{array}$ & $\begin{array}{c}\text { GIC } \\
(p s i-i n)\end{array}$ & $\begin{array}{c}\mathrm{MBT} \\
(\text { ) }\end{array}$ & $\begin{array}{l}\mathrm{CC} \\
()\end{array}$ & $\begin{array}{c}\mathrm{MCC} \\
()\end{array}$ & $\begin{array}{c}\text { EQS } \\
()\end{array}$ \\
\hline \multicolumn{8}{|c|}{ Mean Values----------------------------------------------------- } \\
\hline 2.493 & 0.604 & 7.785 & $\mathrm{NL}$ & 2.759 & 2.623 & 2.644 & 2.016 \\
\hline 2.493 & 0.617 & 7.778 & vis & 2.819 & 2.697 & 2.621 & 2.06 \\
\hline 2.493 & $\mathrm{NaN}$ & $\mathrm{NaN}$ & $5 \%$ & NaN & $\mathrm{NaN}$ & $\mathrm{NaN}$ & $\mathrm{N}$ \\
\hline Standard & Deviatic & $n s-----$ & & & & ------ & ---- \\
\hline $2.54 e-01$ & 0.164 & 0.5327 & $\mathrm{NL}$ & 0.564 & 0.538 & 0.573 & 0.803 \\
\hline $2.54 e-01$ & 0.144 & 0.7153 & vis & 0.526 & 0.495 & 0.489 & 0.670 \\
\hline $2.54 e-01$ & 0.164 & $\mathrm{NaN}$ & $5 \%$ & $\mathrm{NaN}$ & $\mathrm{NaN}$ & $\mathrm{NaN}$ & $\mathrm{NaN}$ \\
\hline \multicolumn{8}{|c|}{ Coefficients of Variation $\left(\frac{\circ}{0}\right)------------------------------------$} \\
\hline $1.02 e+01$ & 27.06 & 6.842 & $\mathrm{NL}$ & 20.44 & 20.50 & 21.68 & 39.83 \\
\hline $1.02 e+01$ & 23.26 & 9.197 & vis & 18.67 & 18.36 & 18.64 & 32.51 \\
\hline $1.02 e+01$ & $\mathrm{NaN}$ & $\mathrm{NaN}$ & $5 \div$ & $\mathrm{NaN}$ & $\mathrm{NaN}$ & $\mathrm{NaN}$ & $\mathrm{NaN}$ \\
\hline
\end{tabular}

Crack Propagation Values

$\begin{array}{lcccc} & \text { GIC-MBT } & \text { GIC-CC } & \text { GIC-MCC } & \text { GIC-EQS } \\ -----------------1 & 2.811 & 2.828 & 2.269 \\ \text { Mean Values } & 2.944 & 2.86 & 0.254 \\ \text { Standard Deviations } & 0.267 & 0.262 & 0.283 & 0.254 \% \\ \text { Coefficients of Variation } & 9.198 \% & 9.198 \% & 9.831 \% & 11.14 \%\end{array}$

Slopes and Intercepts

$\begin{array}{lccc} & \text { Delta } & \text { n } & \text { A1 } \\ ------------------------------------------- \\ \text { Mean Values } & -7.487 e-017 & 2.872 & 47.44 \\ \text { Standard Deviations } & 1.83 e-016 & 0.107 & 4.33 \\ \text { Coefficients of Variation } & -244.9 \% & 3.736 \% & 9.124 \%\end{array}$




\section{H.2 Plate}

T $\begin{array}{llllllllll} & \mathrm{S} & \mathrm{T} & \mathrm{N} & \mathrm{U} & \mathrm{M} & \mathrm{B} & \mathrm{E} & \mathrm{R} & \text { : }\end{array}$

7-1

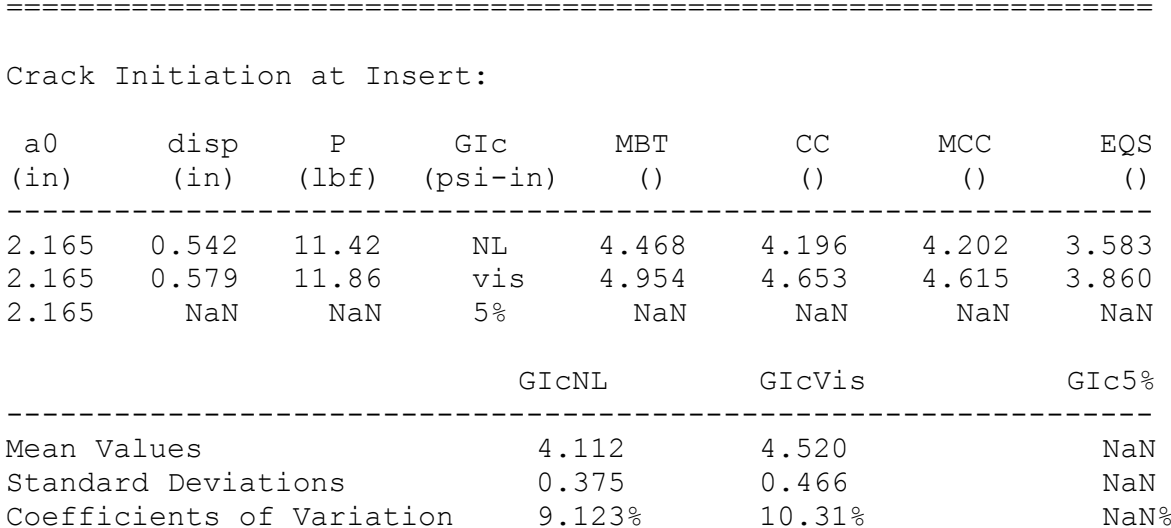

Crack Initiation at Precrack:

\begin{tabular}{|c|c|c|c|c|c|c|c|}
\hline $\begin{array}{c}\text { ap } \\
(\mathrm{in})\end{array}$ & $\begin{array}{l}\text { disp } \\
\text { (in) }\end{array}$ & $\begin{array}{c}\mathrm{P} \\
(1 \mathrm{bf})\end{array}$ & $\begin{array}{c}\text { GIC } \\
(p s i-i n)\end{array}$ & $\begin{array}{c}\text { MBT } \\
()\end{array}$ & $\begin{array}{l}\mathrm{CC} \\
()\end{array}$ & $\begin{array}{c}\mathrm{MCC} \\
()\end{array}$ & \\
\hline .559 & 0.746 & 8.685 & $\mathrm{NL}$ & 3.956 & 3.715 & 3.608 & \\
\hline .559 & 0.816 & 9.326 & vis & 4.647 & 4.364 & 3.993 & \\
\hline 2.559 & NaN & NaN & $5 \%$ & NaN & NaN & $\mathrm{NaN}$ & \\
\hline
\end{tabular}

\begin{tabular}{|c|c|c|c|}
\hline & GICNL & GICVis & GIC 5\% \\
\hline----------- & -------- & ------ & ------ \\
\hline Mean Values & 3.543 & 4.041 & $\mathrm{NaN}$ \\
\hline Standard Deviations & 0.457 & 0.644 & $\mathrm{NaN}$ \\
\hline Coefficients of Variation & $12.9 \%$ & $15.94 \%$ & $\mathrm{NaN} \%$ \\
\hline
\end{tabular}

Crack Propagation Values:

\begin{tabular}{|c|c|c|c|c|c|c|c|}
\hline $\begin{array}{l}\mathrm{ap} \\
(\mathrm{in})\end{array}$ & $\begin{array}{l}\text { disp } \\
(i n)\end{array}$ & $\begin{array}{c}P \\
(1 \mathrm{bf})\end{array}$ & $\begin{array}{c}\text { GIC } \\
(p s i-i n)\end{array}$ & $\begin{array}{c}\mathrm{MBT} \\
()\end{array}$ & $\begin{array}{l}\mathrm{CC} \\
()\end{array}$ & $\begin{array}{c}\mathrm{MCC} \\
()\end{array}$ & $\begin{array}{r}E Q S \\
(\text { ) }\end{array}$ \\
\hline & & & & & & & \\
\hline 2.638 & 0.889 & 9.595 & Prop & 5.052 & 4.745 & 4.632 & 3.753 \\
\hline 3.228 & 1.276 & 8.072 & Prop & 4.986 & 4.682 & 4.681 & 3.977 \\
\hline 3.819 & 1.631 & 6.635 & Prop & 4.428 & 4.158 & 4.245 & 3.761 \\
\hline 4. 291 & 2.141 & 6.052 & Prop & 4.718 & 4.431 & 4.502 & 3.95 \\
\hline 4.350 & 2.352 & 6.296 & Prop & 5.319 & 4.995 & 5.053 & 4.394 \\
\hline
\end{tabular}

\begin{tabular}{|c|c|c|c|c|}
\hline & GIC-MBT & $\mathrm{IC}-\mathrm{CC}$ & GIC-MCC & GIC-EQS \\
\hline Mean Values & 4.901 & 4.602 & 4.623 & 3.967 \\
\hline Standard Deviations & 0.340 & 0.319 & 0.294 & 0.260 \\
\hline Coefficients of Variation & $6.938 \%$ & $6.938 \%$ & $6.357 \%$ & $6.558 \%$ \\
\hline
\end{tabular}




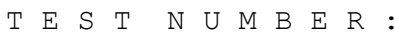

$7-2$

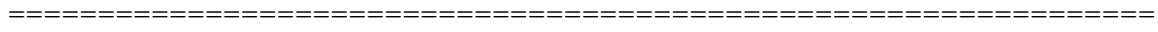

Crack Initiation at Insert:

\begin{tabular}{|c|c|c|c|c|c|c|c|}
\hline $\begin{array}{c}\mathrm{a} 0 \\
(\mathrm{in})\end{array}$ & $\begin{array}{l}\text { disp } \\
(\text { in) }\end{array}$ & $\begin{array}{c}P \\
(1 \mathrm{bf})\end{array}$ & $\begin{array}{c}\text { GIC } \\
(p s i-i n)\end{array}$ & $\begin{array}{c}\mathrm{MBT} \\
()\end{array}$ & $\begin{array}{l}\mathrm{CC} \\
()\end{array}$ & $\begin{array}{c}\mathrm{MCC} \\
()\end{array}$ & $\begin{array}{c}E Q S \\
()\end{array}$ \\
\hline & -----1 & ------ & -------- & ------ & ------- & ------ & ------ \\
\hline 2.165 & 0.515 & 13.21 & NL & 4.734 & 4.298 & 4.148 & 3.293 \\
\hline 2.165 & 0.562 & 14.22 & vis & 5.558 & 5.046 & 4.847 & 3.812 \\
\hline 2.165 & NaN & NaN & $5 \%$ & $\mathrm{NaN}$ & $\mathrm{NaN}$ & $\mathrm{NaN}$ & $\mathrm{NaN}$ \\
\hline \multicolumn{7}{|c|}{ GICNL } & GIC $5 \%$ \\
\hline 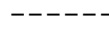 & -- & 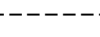 & ------- & 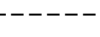 & ------- & 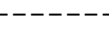 & ------ \\
\hline \multicolumn{5}{|c|}{ Mean Values } & \multicolumn{2}{|l|}{4.816} & $\mathrm{NaN}$ \\
\hline \multicolumn{3}{|c|}{ Standard Deviations } & \multicolumn{2}{|c|}{0.604} & \multicolumn{2}{|l|}{0.733} & $\mathrm{NaN}$ \\
\hline \multicolumn{3}{|c|}{ Coefficients of Var } & on & $66 \%$ & $15.22 \%$ & & $\mathrm{NaN} \stackrel{\circ}{2}$ \\
\hline
\end{tabular}

Crack Initiation at Precrack:

\begin{tabular}{|c|c|c|c|c|c|c|c|}
\hline $\begin{array}{c}\text { ap } \\
(i n)\end{array}$ & $\begin{array}{l}\text { disp } \\
\text { (in) }\end{array}$ & $\begin{array}{c}P \\
(1 \mathrm{bf})\end{array}$ & $\begin{array}{c}\text { GIC } \\
(p s i-i n)\end{array}$ & $\begin{array}{c}\mathrm{MBT} \\
()\end{array}$ & $\begin{array}{l}\mathrm{CC} \\
()\end{array}$ & $\begin{array}{c}\mathrm{MCC} \\
()\end{array}$ & $\begin{array}{r}E Q S \\
(\text { ) }\end{array}$ \\
\hline & & & & ----- & ----- & ----- & \\
\hline 3.110 & 0.902 & 8.673 & $\mathrm{NL}$ & 3.788 & 3.439 & 3.438 & 2.927 \\
\hline 3.110 & 0.963 & 9.134 & vis & 4.260 & 3.867 & 3.669 & 3.096 \\
\hline 3.110 & $\mathrm{NaN}$ & $\mathrm{NaN}$ & $5 \%$ & NaN & $\mathrm{NaN}$ & $\mathrm{NaN}$ & $\mathrm{NaN}$ \\
\hline
\end{tabular}

\begin{tabular}{|c|c|c|c|}
\hline & GICNL & GICVis & GIC5\% \\
\hline Mean Values & 3.398 & 3.723 & $\mathrm{NaN}$ \\
\hline Standard Deviations & 0.355 & 0.485 & $\mathrm{NaN}$ \\
\hline Coefficients of Variation & $10.43 \%$ & $13.02 \%$ & $\mathrm{NaN} \circ$ \\
\hline
\end{tabular}

Crack Propagation Values:

\begin{tabular}{|c|c|c|c|c|c|c|c|}
\hline $\begin{array}{l}\mathrm{ap} \\
(\mathrm{in})\end{array}$ & $\begin{array}{l}\text { disp } \\
(\text { in) }\end{array}$ & $\begin{array}{c}P \\
(1 \mathrm{bf})\end{array}$ & $\begin{array}{c}\text { GIC } \\
(p s i-i n)\end{array}$ & $\begin{array}{c}\text { MBT } \\
()\end{array}$ & $\begin{array}{l}\mathrm{CC} \\
()\end{array}$ & $\begin{array}{c}\mathrm{MCC} \\
()\end{array}$ & $\begin{array}{r}E Q S \\
(\text { ) }\end{array}$ \\
\hline 3.898 & 1.468 & 7.617 & Prop & 4.320 & 3.922 & 4.000 & 3.54 \\
\hline 4.843 & 2.114 & 5.933 & Prop & 3.900 & 3.541 & 3.656 & 3.322 \\
\hline
\end{tabular}

\begin{tabular}{|c|c|c|c|c|}
\hline & GIC-MBT & $\mathrm{GIC}-\mathrm{CC}$ & GIC-MCC & GIC-EQS \\
\hline & & & ------ & o 0 \\
\hline ean Values & 4.110 & 3.732 & 3.828 & 3.434 \\
\hline tandard Deviations & 0.297 & 0.270 & 0.244 & 0.159 \\
\hline Coefficients of Variation & $7.223 \%$ & $7.223 \%$ & $6.361 \%$ & $4.634 \%$ \\
\hline
\end{tabular}


T E $\quad S \quad T \quad N \quad U$ M $B$ E R :

$7-3$

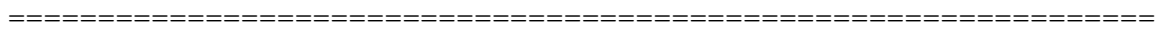

Crack Initiation at Insert:

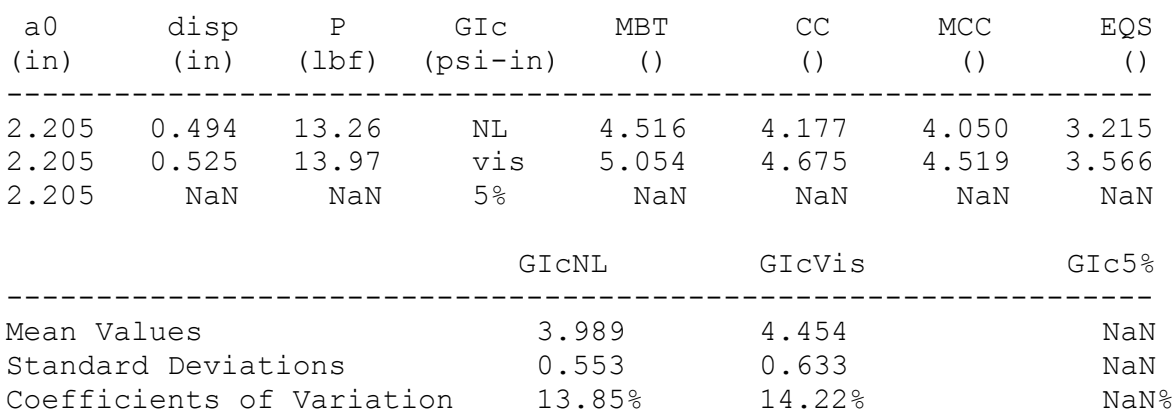

Crack Initiation at Precrack:

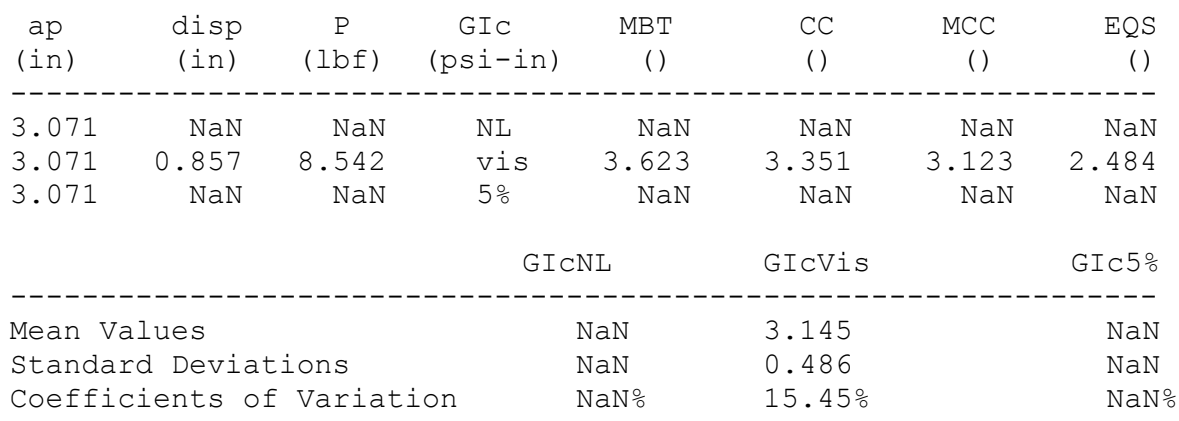

Crack Propagation Values:

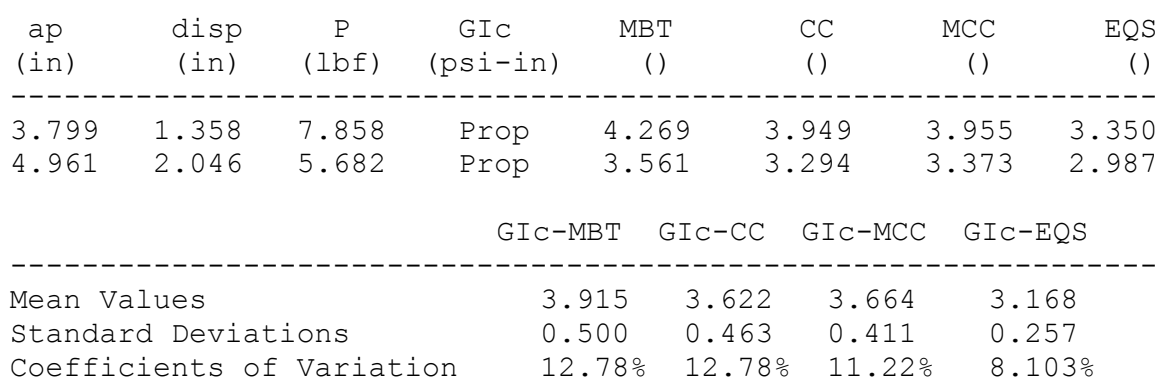


T E $\quad S \quad T \quad N \quad U$ M $B$ E R :

$7-4$

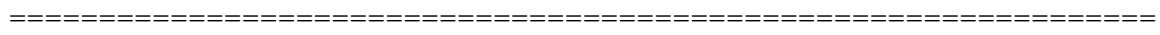

Crack Initiation at Insert:

\begin{tabular}{|c|c|c|c|c|c|c|c|}
\hline $\begin{array}{c}\mathrm{a} 0 \\
(\mathrm{in})\end{array}$ & $\begin{array}{l}\text { disp } \\
(i n)\end{array}$ & $\begin{array}{c}\mathrm{P} \\
(1 \mathrm{bf})\end{array}$ & $\begin{array}{c}\text { GIC } \\
(p s i-i n)\end{array}$ & $\begin{array}{r}\mathrm{MBT} \\
()\end{array}$ & $\begin{array}{l}\text { CC } \\
()\end{array}$ & $\begin{array}{c}\mathrm{MCC} \\
()\end{array}$ & $\begin{array}{c}\text { EQS } \\
()\end{array}$ \\
\hline \multicolumn{8}{|c|}{ 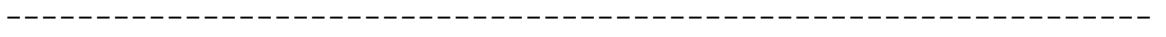 } \\
\hline 2.165 & 0.470 & 12.01 & vis & 4.062 & 3.632 & 3.537 & 2.857 \\
\hline 2.165 & $\mathrm{NaN}$ & $\mathrm{NaN}$ & $5 \%$ & $\mathrm{NaN}$ & $\mathrm{NaN}$ & $\mathrm{NaN}$ & $\mathrm{NaN}$ \\
\hline \multicolumn{5}{|c|}{ GICNL } & GIcVis & & GIC5\% \\
\hline \multicolumn{8}{|c|}{ 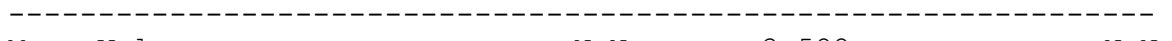 } \\
\hline \multicolumn{4}{|c|}{ Mean Values } & \multicolumn{3}{|c|}{$\mathrm{NaN} \quad 3.522$} & \\
\hline \multicolumn{4}{|c|}{ Standard Deviations } & $\mathrm{NaN}$ & 0.499 & & $\mathrm{NaN}$ \\
\hline \multicolumn{4}{|c|}{ Coefficients of Variation } & $\mathrm{NaN} \%$ & $14.16 \%$ & & $\mathrm{aN} ㅇ$ \\
\hline
\end{tabular}

Crack Initiation at Precrack:

\begin{tabular}{|c|c|c|c|c|c|c|c|}
\hline $\begin{array}{c}\text { ap } \\
(i n)\end{array}$ & $\begin{array}{l}\text { disp } \\
(\text { in) }\end{array}$ & $\begin{array}{c}P \\
(1 \mathrm{bf})\end{array}$ & $\begin{array}{c}\text { GIC } \\
(p s i-i n)\end{array}$ & $\begin{array}{c}\mathrm{MBT} \\
(\mathbf{)}\end{array}$ & $\begin{array}{l}\mathrm{CC} \\
()\end{array}$ & $\begin{array}{c}\mathrm{MCC} \\
()\end{array}$ & \\
\hline 2.441 & 0.630 & 10.08 & $\mathrm{NL}$ & 4.052 & 3.623 & 3.404 & 2 \\
\hline 2.441 & 0.676 & 10.82 & vis & 4.665 & 4.171 & 3.749 & 2.8 \\
\hline 2.441 & $\mathrm{NaN}$ & $\mathrm{NaN}$ & $5 \%$ & $\mathrm{NaN}$ & NaN & $\mathrm{NaN}$ & \\
\hline
\end{tabular}

\begin{tabular}{|c|c|c|c|}
\hline & GICNL & GICVis & GIC5\% \\
\hline Mean Values & 3.409 & 3.850 & $\mathrm{NaN}$ \\
\hline Standard Deviations & 0.628 & 0.784 & $\mathrm{NaN}$ \\
\hline Coefficients of Variation & $18.43 \%$ & $20.37 \%$ & $\mathrm{NaN} \circ$ \\
\hline
\end{tabular}

Crack Propagation Values:

\begin{tabular}{|c|c|c|c|c|c|c|c|}
\hline $\begin{array}{l}\mathrm{ap} \\
(\mathrm{in})\end{array}$ & $\begin{array}{l}\text { disp } \\
(\text { in) }\end{array}$ & $\begin{array}{c}\mathrm{P} \\
(1 \mathrm{bf})\end{array}$ & $\begin{array}{c}\text { GIC } \\
(p s i-i n)\end{array}$ & $\begin{array}{c}\mathrm{MBT} \\
()\end{array}$ & $\begin{array}{l}\mathrm{CC} \\
()\end{array}$ & $\begin{array}{c}\mathrm{MCC} \\
()\end{array}$ & $\begin{array}{c}\text { EQS } \\
(\text { ) }\end{array}$ \\
\hline 3.209 & 1.090 & 9.003 & Prop & 4.763 & 4.259 & 4.219 & 3.52 \\
\hline 4.409 & 1.794 & 6.464 & Prop & 4.097 & 3.663 & 3.782 & 3.431 \\
\hline
\end{tabular}

\begin{tabular}{|c|c|c|c|c|}
\hline & GIC-MBT & $\mathrm{GIC}-\mathrm{CC}$ & GIC-MCC & GIC-EQS \\
\hline & & & & \\
\hline Mean Val & 4.430 & 3.961 & 4.000 & 3.479 \\
\hline Standard Deviations & 0.471 & 0.421 & 0.310 & 0.067 \\
\hline Coefficients of Variation & $10.64 \%$ & $10.64 \%$ & $7.74 \%$ & $1.926 \%$ \\
\hline
\end{tabular}


T E $\quad S \quad T \quad N \quad U$ M $B$ E R :

$7-5$

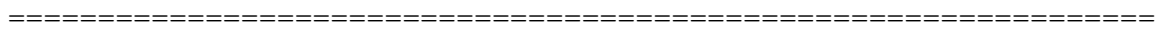

Crack Initiation at Insert:

\begin{tabular}{|c|c|c|c|c|c|c|c|}
\hline $\begin{array}{c}\mathrm{a} 0 \\
(\mathrm{in})\end{array}$ & $\begin{array}{l}\text { disp } \\
(i n)\end{array}$ & $\begin{array}{c}\mathrm{P} \\
(\mathrm{lbf})\end{array}$ & $\begin{array}{c}\text { GIC } \\
(p s i-i n)\end{array}$ & $\begin{array}{c}\text { MBT } \\
(\text { ) }\end{array}$ & $\begin{array}{l}\text { CC } \\
()\end{array}$ & $\begin{array}{c}\mathrm{MCC} \\
()\end{array}$ & $\begin{array}{c}\text { EQS } \\
()\end{array}$ \\
\hline \multicolumn{8}{|c|}{ 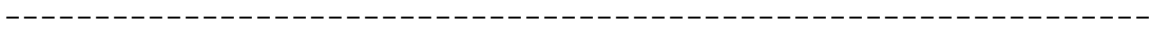 } \\
\hline 2.165 & 0.511 & 12.04 & NL & 4.211 & 3.861 & 3.761 & 3.321 \\
\hline 2.165 & 0.540 & 12.58 & vis & 4.649 & 4.263 & 4.138 & 3.625 \\
\hline 2.165 & $\mathrm{NaN}$ & $\mathrm{NaN}$ & $5 \%$ & $\mathrm{NaN}$ & $\mathrm{NaN}$ & $\mathrm{NaN}$ & $\mathrm{NaN}$ \\
\hline & & & GIC & & & & GIC $5 \%$ \\
\hline & & & -1 & . & ------ & & \\
\hline \multicolumn{5}{|c|}{ Mean Values } & \multicolumn{2}{|l|}{4.169} & $\mathrm{NaN}$ \\
\hline \multicolumn{3}{|c|}{ Standard Deviations } & & 67 & \multicolumn{2}{|l|}{0.423} & $\mathrm{NaN}$ \\
\hline \multicolumn{3}{|c|}{ Coefficients of Varia } & on & $76 \%$ & \multicolumn{2}{|l|}{$10.14 \%$} & $\mathrm{NaN} \stackrel{\circ}{2}$ \\
\hline
\end{tabular}

Crack Initiation at Precrack:

\begin{tabular}{|c|c|c|c|c|c|c|c|}
\hline $\begin{array}{l}\text { ap } \\
(i n)\end{array}$ & $\begin{array}{l}\text { disp } \\
(\text { in) }\end{array}$ & $\begin{array}{c}P \\
(1 \mathrm{bf})\end{array}$ & $\begin{array}{c}\text { GIC } \\
(p s i-i n)\end{array}$ & $\begin{array}{c}\mathrm{MBT} \\
(\mathrm{)}\end{array}$ & $\begin{array}{l}\mathrm{CC} \\
()\end{array}$ & $\begin{array}{c}\mathrm{MCC} \\
()\end{array}$ & \\
\hline 2.559 & 0.665 & 9.882 & $\mathrm{NL}$ & 3.806 & 3.490 & 3.447 & 3. \\
\hline 2.559 & 0.728 & 10.42 & vis & 4.395 & 4.029 & 3.759 & 3.3 \\
\hline 2.559 & $\mathrm{NaN}$ & $\mathrm{NaN}$ & $5 \%$ & $\mathrm{NaN}$ & NaN & NaN & \\
\hline
\end{tabular}

\begin{tabular}{|c|c|c|c|}
\hline & GICNL & GICVis & GIC $5 \%$ \\
\hline & & & \\
\hline Mean Values & 3.468 & 3.877 & $\mathrm{NaN}$ \\
\hline Standard Deviations & 0.278 & 0.450 & $\mathrm{NaN}$ \\
\hline Coefficients of Variation & $8.01 \%$ & $11.61 \%$ & $\mathrm{NaN} \%$ \\
\hline
\end{tabular}

Crack Propagation Values:

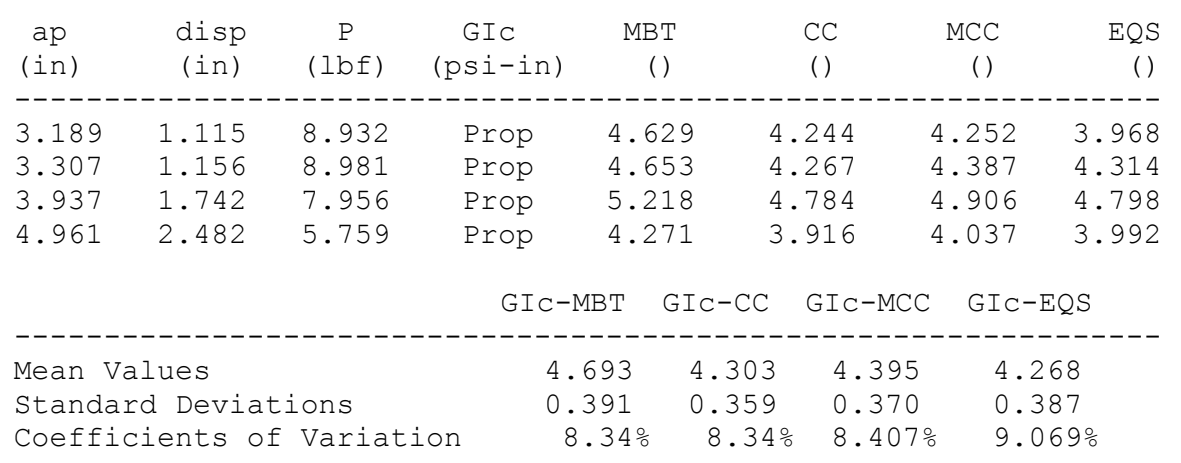


P L A T E

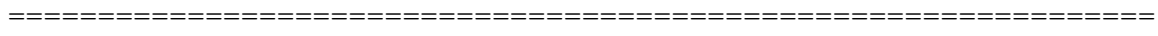

Crack Initiation at Insert

\begin{tabular}{|c|c|c|c|c|c|c|c|}
\hline $\begin{array}{c}\mathrm{a} 0 \\
(\mathrm{in})\end{array}$ & $\begin{array}{l}\text { disp } \\
(\text { in) }\end{array}$ & $\begin{array}{c}\mathrm{P} \\
(1 \mathrm{bf})\end{array}$ & $\begin{array}{c}\text { GIC } \\
(\operatorname{psi-in)}\end{array}$ & $\begin{array}{c}\mathrm{MBT} \\
()\end{array}$ & $\begin{array}{l}\mathrm{CC} \\
()\end{array}$ & $\begin{array}{c}\mathrm{MCC} \\
()\end{array}$ & $\begin{array}{c}E Q S \\
(\text { ) }\end{array}$ \\
\hline \multicolumn{8}{|c|}{ Mean Values------------------------------------------------------- } \\
\hline 2.173 & 0.516 & 12.48 & NL & 4.482 & 4.133 & 4.040 & 3.353 \\
\hline 2.173 & 0.535 & 12.93 & vis & 4.856 & 4.454 & 4.331 & 3.544 \\
\hline 2.173 & $\mathrm{NaN}$ & $\mathrm{NaN}$ & $5 \%$ & $\mathrm{NaN}$ & $\mathrm{NaN}$ & $\mathrm{NaN}$ & $\mathrm{NaN}$ \\
\hline Standard & \multicolumn{7}{|c|}{ Deviations ---------------------------------------------} \\
\hline $1.79 e-02$ & 0.020 & 0.907 & $\mathrm{NL}$ & 0.215 & 0.189 & 0.196 & 0.160 \\
\hline $1.79 e-02$ & 0.042 & 1.100 & vis & 0.551 & 0.537 & 0.512 & 0.403 \\
\hline $1.79 e-02$ & 0.020 & $\mathrm{NaN}$ & $5 \%$ & $\mathrm{NaN}$ & $\mathrm{NaN}$ & $\mathrm{NaN}$ & $\mathrm{NaN}$ \\
\hline \multicolumn{8}{|c|}{ Coefficients of Variation $\left(\frac{\circ}{\circ}\right)-------------------------------------$} \\
\hline $8.23 e-01$ & 3.855 & 7.263 & $\mathrm{NL}$ & 4.791 & 4.575 & 4.861 & 4.764 \\
\hline $3 e-01$ & 7.825 & 8.526 & Vis & 11.35 & 12.05 & 11.83 & 11.38 \\
\hline $3 e-01$ & $\mathrm{NaN}$ & $\mathrm{NaN}$ & $5 \%$ & $\mathrm{NaN}$ & $\mathrm{NaN}$ & $\mathrm{NaN}$ & \\
\hline
\end{tabular}

Crack Initiation at Precrack

\begin{tabular}{|c|c|c|c|c|c|c|c|}
\hline $\begin{array}{l}\operatorname{ap} \\
(i n)\end{array}$ & $\begin{array}{c}\text { disp } \\
(i n)\end{array}$ & $\begin{array}{c}\mathrm{P} \\
(1 \mathrm{bf})\end{array}$ & $\begin{array}{c}\text { GIC } \\
(p s i-i n)\end{array}$ & $\begin{array}{c}\text { MBT } \\
()\end{array}$ & $\begin{array}{l}\mathrm{CC} \\
()\end{array}$ & $\begin{array}{c}\mathrm{MCC} \\
()\end{array}$ & $\begin{array}{r}\text { EQS } \\
()\end{array}$ \\
\hline \multicolumn{8}{|c|}{ Mean Values----------------------------------------------------- } \\
\hline 2.748 & 0.736 & 9.330 & NL & 3.901 & 3.567 & 3.474 & 2.876 \\
\hline 2.748 & 0.808 & 9.648 & vis & 4.318 & 3.957 & 3.658 & 2.977 \\
\hline 2.748 & NaN & $\mathrm{NaN}$ & $5 \%$ & NaN & NaN & NaN & $\mathrm{NaN}$ \\
\hline Standard & Deviati & $n S----$ & & 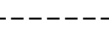 & $x^{-1-----1}$ & ------ & ------ \\
\hline $3.17 e-01$ & 0.121 & 0.756 & $\mathrm{NL}$ & 0.126 & 0.1256 & 0.091 & 0.236 \\
\hline $3.17 e-01$ & 0.112 & 0.942 & vis & 0.425 & 0.3846 & 0.323 & 0.332 \\
\hline $3.17 e-01$ & 0.121 & $\mathrm{NaN}$ & $5 \%$ & $\mathrm{NaN}$ & $\mathrm{NaN}$ & $\mathrm{NaN}$ & $\mathrm{NaN}$ \\
\hline \multicolumn{8}{|c|}{ Coefficients of Variation $\left(\frac{\circ}{\circ}\right)----------------------------------1$} \\
\hline $1.15 e+01$ & 16.45 & 8.104 & NL & 3.228 & 3.522 & 2.630 & 8.215 \\
\hline $1.15 e+01$ & 13.88 & 9.767 & vis & 9.835 & 9.722 & 8.824 & 11. \\
\hline $1.15 e+01$ & $\mathrm{NaN}$ & $\mathrm{NaN}$ & $5 \%$ & $\mathrm{NaN}$ & NaN & $\mathrm{NaN}$ & $\mathrm{NaN}$ \\
\hline
\end{tabular}

Crack Propagation Values

\begin{tabular}{|c|c|c|c|c|}
\hline------------------------- & $\begin{array}{l}\text { GIC-MBT } \\
---------\end{array}$ & $\begin{array}{l}\mathrm{GIC}-\mathrm{CC} \\
--------\end{array}$ & $\begin{array}{l}\text { GIC }-\mathrm{MCC} \\
--------\end{array}$ & $\begin{array}{l}\mathrm{GIC}-\mathrm{EQS} \\
-----------\end{array}$ \\
\hline Mean Values & 4.410 & 4.044 & 4.102 & 3.663 \\
\hline Standard Deviations & 0.400 & 0.366 & 0.326 & 0.226 \\
\hline Coefficients of Variation & $9.184 \%$ & $9.184 \%$ & $8.018 \%$ & $6.058 \%$ \\
\hline
\end{tabular}

Slopes and Intercepts

$\begin{array}{lccc} & \text { Delta } & \text { n } & \text { A1 } \\ ---------1 & & \\ \text { Mean Values } & -1.765 e-016 & 2.75 & 50.3 \\ \text { Standard Deviations } & 2.78 e-016 & 0.051 & 1.19 \\ \text { Coefficients of Variation } & -157.6 \% & 1.859 \% & 2.371 \%\end{array}$




\section{H.3 Plate}

T

$8-1$

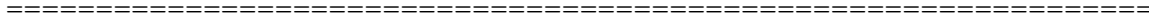

Crack Initiation at Insert:

$\begin{array}{lllllll}\text { a0 disp } & P & \text { GIC } & \text { MBT } & \text { CC } & \text { MCC } & \text { EQS }\end{array}$

(in) (in) (lbf) (psi-in) $\quad() \quad$ ()

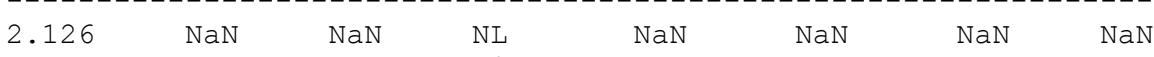

$\begin{array}{lllllllll}2.126 & 0.470 & 9.616 & \text { vis } & 3.304 & 3.121 & 3.071 & 2.792\end{array}$

$2.126 \mathrm{NaN} \quad \mathrm{NaN} \quad 5 \%$ NaN $\quad$ NaN $\quad$ NaN

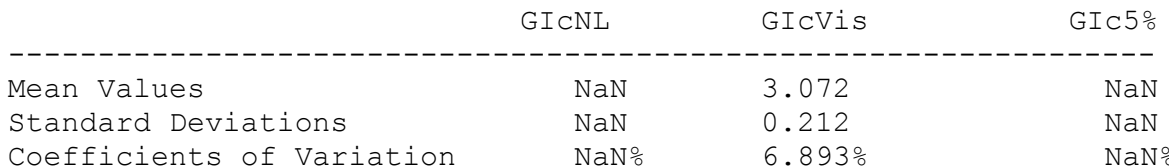

Crack Initiation at Precrack:

\begin{tabular}{|c|c|c|c|c|c|c|c|}
\hline $\begin{array}{c}\mathrm{ap} \\
(\mathrm{in})\end{array}$ & $\begin{array}{l}\text { disp } \\
(i n)\end{array}$ & $\begin{array}{c}\mathrm{P} \\
(\mathrm{lbf})\end{array}$ & $\begin{array}{c}\text { GIC } \\
(\mathrm{psi}-i n)\end{array}$ & $\begin{array}{c}\mathrm{MBT} \\
(\text { ) }\end{array}$ & $\begin{array}{l}\mathrm{CC} \\
()\end{array}$ & $\begin{array}{c}\mathrm{MCC} \\
()\end{array}$ & \\
\hline 618 & 0.753 & 8.225 & $\mathrm{NL}$ & 3.674 & 3.471 & 3.412 & \\
\hline 2.618 & 0.778 & 8.444 & vis & 3.898 & 3.683 & 3.447 & \\
\hline 2.618 & $\mathrm{NaN}$ & $\mathrm{NaN}$ & $5 \%$ & $\mathrm{NaN}$ & NaN & $\mathrm{NaN}$ & \\
\hline
\end{tabular}

\begin{tabular}{|c|c|c|c|}
\hline & GICNL & GIcVis & GIC $5 \%$ \\
\hline & -------- & -------- & ------ \\
\hline Mean Values & 3.413 & 3.536 & $\mathrm{NaN}$ \\
\hline Standard Deviations & 0.239 & 0.335 & $\mathrm{NaN}$ \\
\hline Coefficients of Variation & $6.991 \%$ & $9.488 \%$ & $\mathrm{NaN} \circ$ \\
\hline
\end{tabular}

Crack Propagation Values:

\begin{tabular}{|c|c|c|c|c|c|c|c|}
\hline $\begin{array}{c}\text { ap } \\
(i n)\end{array}$ & $\begin{array}{l}\text { disp } \\
\text { (in) }\end{array}$ & $\begin{array}{c}P \\
(1 \mathrm{bf})\end{array}$ & $\begin{array}{c}\text { GIC } \\
(\mathrm{psi}-i n)\end{array}$ & $\begin{array}{c}\mathrm{MBT} \\
()\end{array}$ & $\begin{array}{c}\mathrm{CC} \\
()\end{array}$ & $\begin{array}{c}\mathrm{MCC} \\
(\text { ) }\end{array}$ & $\begin{array}{c}E Q S \\
\text { () }\end{array}$ \\
\hline 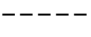 & --- & -- & ----- & ----- & ------ & ----- & \\
\hline 3.602 & 1.284 & 5.853 & Prop & 3.239 & 3.060 & 3.094 & \\
\hline
\end{tabular}

\begin{tabular}{|c|c|c|c|c|}
\hline & GIC-MBT & $\mathrm{GIC}-\mathrm{CC}$ & GIC-MCC & GIC-EQS \\
\hline & & & ------ & ------ \\
\hline lean Values & 3.239 & 3.060 & 3.094 & 2.970 \\
\hline Standard Deviations & 0 & 0 & 0 & 0 \\
\hline Coefficients of Variation & $0 \%$ & $0 \%$ & $0 \%$ & $0 \%$ \\
\hline
\end{tabular}




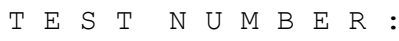

$8-2$

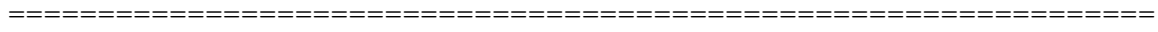

Crack Initiation at Insert:

\begin{tabular}{|c|c|c|c|c|c|c|c|}
\hline $\begin{array}{c}\mathrm{a} 0 \\
(\mathrm{in})\end{array}$ & $\begin{array}{l}\text { disp } \\
(i n)\end{array}$ & $\begin{array}{c}\mathrm{P} \\
(1 \mathrm{bf})\end{array}$ & $\begin{array}{c}\text { GIC } \\
(p s i-i n)\end{array}$ & $\begin{array}{r}\text { MBT } \\
()\end{array}$ & $\begin{array}{l}\text { CC } \\
()\end{array}$ & $\begin{array}{c}\mathrm{MCC} \\
()\end{array}$ & $\begin{array}{c}\text { EQS } \\
()\end{array}$ \\
\hline \multicolumn{8}{|c|}{ 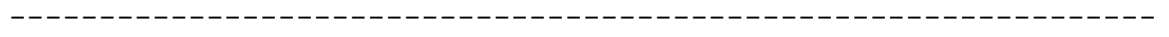 } \\
\hline 2.087 & 0.372 & 9.634 & $\mathrm{NL}$ & 2.544 & 2.232 & 2.130 & 2.167 \\
\hline 2.087 & 0.385 & 9.934 & vis & 2.708 & 2.376 & 2.266 & 2.303 \\
\hline 2.087 & $\mathrm{NaN}$ & $\mathrm{NaN}$ & $5 \%$ & $\mathrm{NaN}$ & $\mathrm{NaN}$ & $\mathrm{NaN}$ & $\mathrm{NaN}$ \\
\hline & & & GIC & & & & GIC $5 \%$ \\
\hline & & & $1-1$ & & ------ & & \\
\hline \multicolumn{5}{|c|}{ Mean Values } & \multicolumn{2}{|l|}{2.413} & \\
\hline \multicolumn{3}{|c|}{ Standard Deviations } & & 89 & \multicolumn{2}{|l|}{0.202} & $\mathrm{NaN}$ \\
\hline \multicolumn{3}{|c|}{ Coefficients of Vari } & on & $12 \%$ & \multicolumn{2}{|l|}{$8.351 \%$} & \\
\hline
\end{tabular}

Crack Initiation at Precrack:

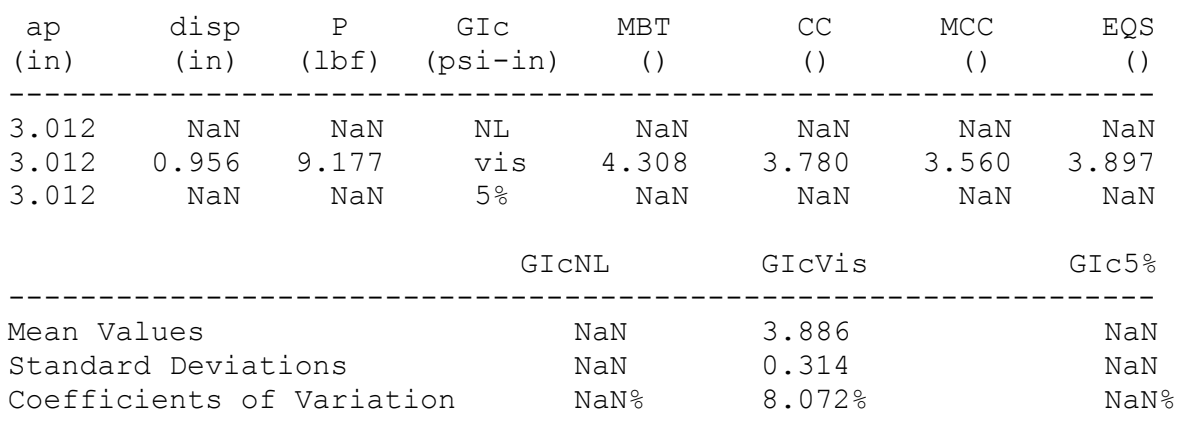

Crack Propagation Values:

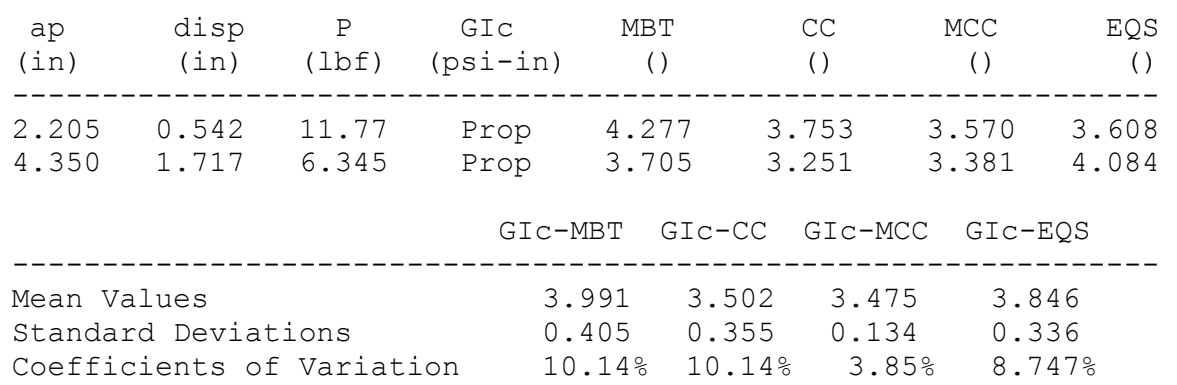




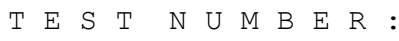

$8-3$

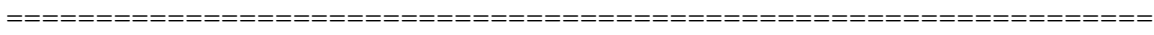

Crack Initiation at Insert:

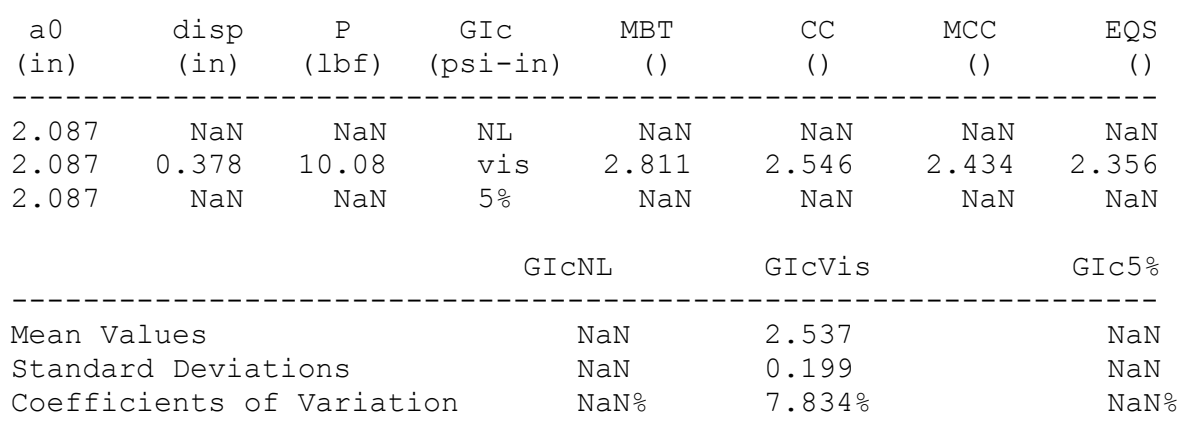

Crack Initiation at Precrack:

\begin{tabular}{|c|c|c|c|c|c|c|c|}
\hline $\begin{array}{l}\text { ap } \\
(i n)\end{array}$ & $\begin{array}{l}\text { disp } \\
(\text { in) }\end{array}$ & $\begin{array}{c}P \\
(1 \mathrm{bf})\end{array}$ & $\begin{array}{c}\text { GIC } \\
(p s i-i n)\end{array}$ & $\begin{array}{c}\mathrm{MBT} \\
()\end{array}$ & $\begin{array}{l}\mathrm{CC} \\
()\end{array}$ & $\begin{array}{c}\mathrm{MCC} \\
()\end{array}$ & EQS \\
\hline 3.189 & 1.092 & 8.887 & $\mathrm{NL}$ & 4.682 & 4.242 & 4.174 & 4.27 \\
\hline 3.189 & 1.156 & 9.299 & vis & 5.184 & 4.697 & 4.331 & 4.4 \\
\hline 3.189 & $\mathrm{NaN}$ & $\mathrm{NaN}$ & $5 \%$ & NaN & NaN & $\mathrm{NaN}$ & \\
\hline
\end{tabular}

\begin{tabular}{|c|c|c|c|}
\hline & GICNL & GICVis & GIC5\% \\
\hline Mean Values & 4.344 & 4.655 & $\mathrm{NaN}$ \\
\hline Standard Deviations & 0.230 & 0.386 & $\mathrm{NaN}$ \\
\hline Coefficients of Variation & $5.284 \%$ & $8.297 \%$ & $\mathrm{NaN} \%$ \\
\hline
\end{tabular}

Crack Propagation Values:

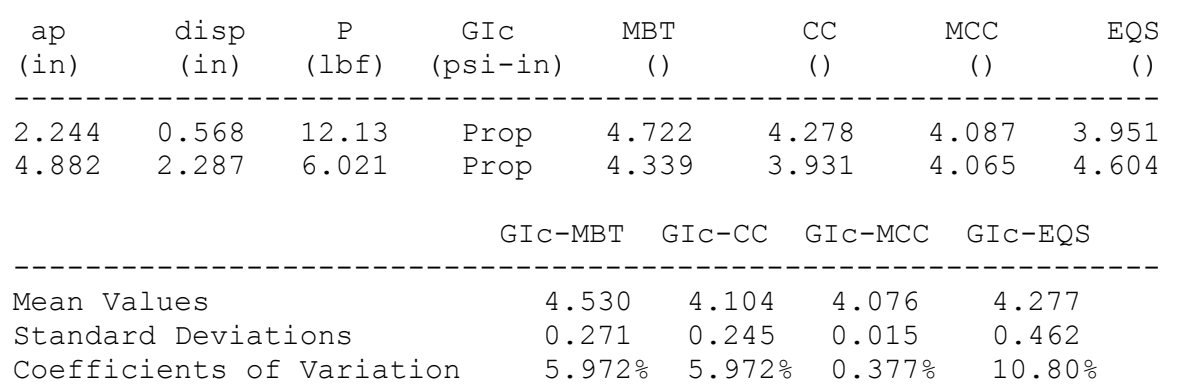


T E S T $\mathrm{S} \quad \mathrm{N}$ U $\mathrm{M}$ B E R : 8-4

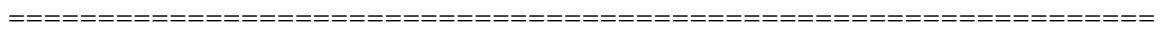

Crack Initiation at Insert:

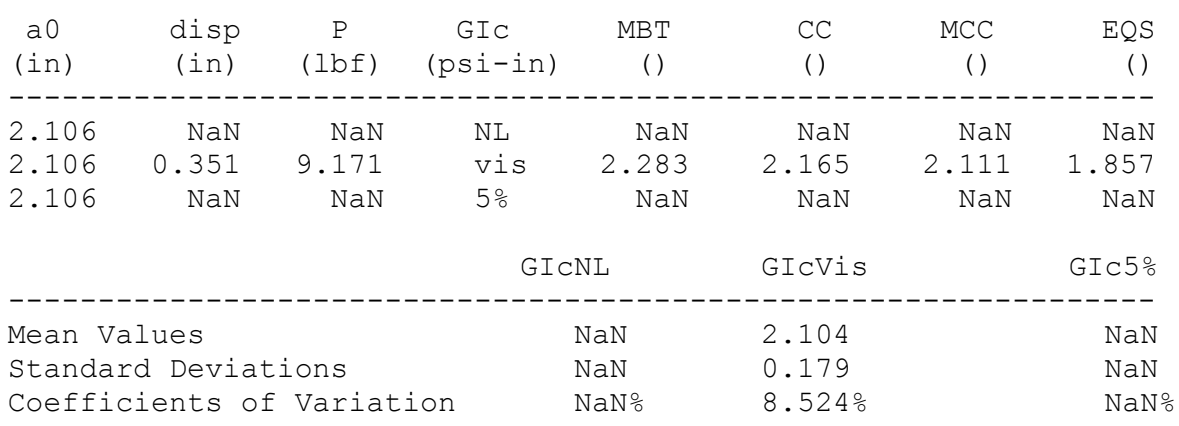

Crack Initiation at Precrack:

\begin{tabular}{|c|c|c|c|c|c|c|c|}
\hline $\begin{array}{c}\text { ap } \\
(i n)\end{array}$ & $\begin{array}{l}\text { disp } \\
(i n)\end{array}$ & $\begin{array}{c}P \\
(1 \mathrm{bf})\end{array}$ & $\begin{array}{c}\text { GIC } \\
(p s i-i n)\end{array}$ & $\begin{array}{c}\mathrm{MBT} \\
(\mathbf{)}\end{array}$ & $\begin{array}{l}\mathrm{CC} \\
()\end{array}$ & $\begin{array}{c}\mathrm{MCC} \\
()\end{array}$ & \\
\hline 2.815 & 0.775 & 8.588 & $\mathrm{NL}$ & 3.534 & 3.351 & 3.281 & 2.9 \\
\hline 2.815 & 0.807 & 8.884 & vis & 3.809 & 3.612 & 3.378 & 2. \\
\hline 2.815 & NaN & $\mathrm{NaN}$ & $5 \%$ & $\mathrm{NaN}$ & NaN & NaN & \\
\hline
\end{tabular}

\begin{tabular}{|c|c|c|c|}
\hline & GICNL & GICVis & GIC $5 \%$ \\
\hline Mean Values & 3269 & 3445 & \\
\hline Standard Deviations & 0.262 & 0.356 & $\mathrm{NaN}$ \\
\hline Coefficients of Variation & $8.019 \%$ & $10.33 \%$ & \\
\hline
\end{tabular}

Crack Propagation Values:

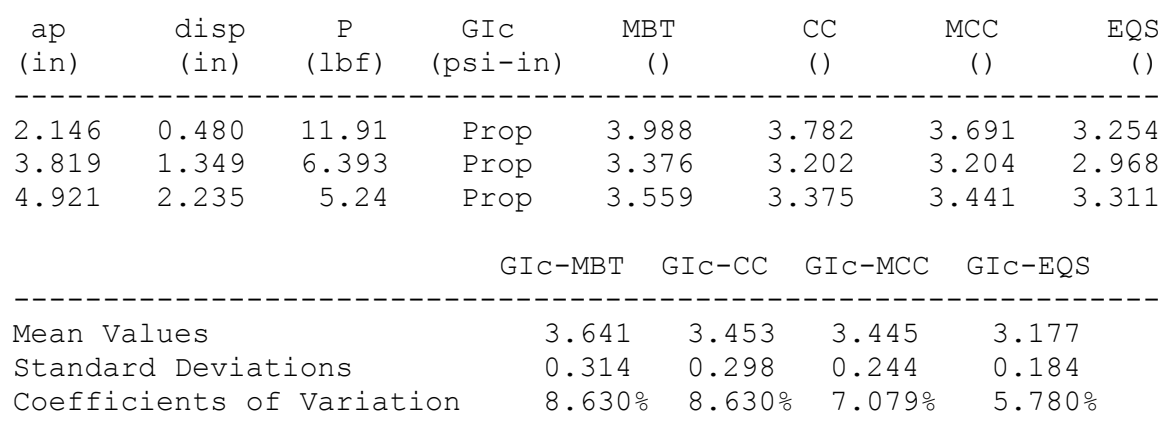


T E $\quad S \quad T \quad N \quad U$ M $B$ E R :

$8-5$

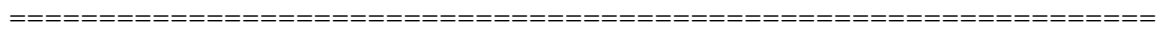

Crack Initiation at Insert:

\begin{tabular}{|c|c|c|c|c|c|c|c|}
\hline $\begin{array}{c}\mathrm{a} 0 \\
(\mathrm{in})\end{array}$ & $\begin{array}{l}\text { disp } \\
(\text { in) }\end{array}$ & $\begin{array}{c}P \\
(1 \mathrm{~b} f)\end{array}$ & $\begin{array}{c}\text { GIC } \\
(p s i-i n)\end{array}$ & $\begin{array}{c}\mathrm{MBT} \\
(\text { ) }\end{array}$ & $\begin{array}{l}\mathrm{CC} \\
()\end{array}$ & $\begin{array}{c}\mathrm{MCC} \\
()\end{array}$ & \\
\hline 26 & 0.469 & 11.09 & $\mathrm{NL}$ & 3.638 & 3.393 & 3.315 & \\
\hline 26 & 0.477 & 11.26 & vis & 3.753 & 3.499 & 3.419 & 2 \\
\hline 26 & NaN & NaN & $5 \%$ & $\mathrm{NaN}$ & $\mathrm{NaN}$ & $\mathrm{NaN}$ & \\
\hline
\end{tabular}

$2.126 \quad \mathrm{NaN} \quad \mathrm{NaN}$ 5\%

GICNL GICVis GIC5\%

\begin{tabular}{|c|c|c|c|}
\hline Mean Values & 3.311 & 3.415 & $\mathrm{NaN}$ \\
\hline Standard Deviations & 0.307 & 0.317 & $\mathrm{NaN}$ \\
\hline Coefficients of Variation & $9.282 \%$ & $9.296 \%$ & $\mathrm{NaN} \circ$ \\
\hline
\end{tabular}

Crack Initiation at Precrack:

\begin{tabular}{|c|c|c|c|c|c|c|c|}
\hline $\begin{array}{c}\mathrm{ap} \\
(\mathrm{in})\end{array}$ & $\begin{array}{l}\text { disp } \\
(\text { in) }\end{array}$ & $\begin{array}{c}P \\
(1 \mathrm{bf})\end{array}$ & $\begin{array}{c}\text { GIC } \\
(\mathrm{psi}-i n)\end{array}$ & $\begin{array}{c}\mathrm{MBT} \\
()\end{array}$ & $\begin{array}{l}\mathrm{CC} \\
()\end{array}$ & $\begin{array}{r}\mathrm{MCC} \\
()\end{array}$ & \\
\hline & -----1 & --- & ---- & ----- & ---- & ---- & \\
\hline 776 & 0.793 & 8.960 & $\mathrm{NL}$ & 3.807 & 3.55 & 3.541 & \\
\hline .776 & 0.833 & 9.354 & vis & 4.172 & 3.891 & 3.697 & \\
\hline .776 & $\mathrm{NaN}$ & $\mathrm{NaN}$ & $5 \%$ & NaN & NaN & $\mathrm{NaN}$ & \\
\hline
\end{tabular}

\begin{tabular}{|c|c|c|c|}
\hline & GICNL & GICVis & GIC $5 \%$ \\
\hline & & & \\
\hline Mean Values & 3.531 & 3.779 & $\mathrm{NaN}$ \\
\hline Standard Deviations & 0.237 & 0.343 & $\mathrm{NaN}$ \\
\hline Coefficients of Variation & $6.712 \%$ & $9.072 \%$ & $\mathrm{NaN} \%$ \\
\hline
\end{tabular}

Crack Propagation Values:

\begin{tabular}{|c|c|c|c|c|c|c|c|}
\hline $\begin{array}{c}a p \\
(i n)\end{array}$ & $\begin{array}{l}\operatorname{disp} \\
(\text { in) }\end{array}$ & $\begin{array}{c}\mathrm{P} \\
(1 \mathrm{~b} f)\end{array}$ & $\begin{array}{c}\text { GIC } \\
(p s i-i n)\end{array}$ & $\begin{array}{c}\text { MBT } \\
()\end{array}$ & $\begin{array}{l}\mathrm{CC} \\
()\end{array}$ & $\begin{array}{c}\mathrm{MCC} \\
()\end{array}$ & $\begin{array}{r}E Q S \\
()\end{array}$ \\
\hline 3.898 & 1.695 & 7.346 & Prop & 4.748 & 4.428 & 4.507 & $4.27^{\prime}$ \\
\hline
\end{tabular}

$\begin{array}{lrrrr} & \text { GIC-MBT } & \text { GIC-CC } & \text { GIC-MCC } & \text { GIC-EQS } \\ --------------------------------------------- \\ \text { Mean Values } & 4.748 & 4.428 & 4.507 & 4.277 \\ \text { Standard Deviations } & 0 & 0 & 0 & 0 \\ \text { Coefficients of Variation } & 0 \% & 0 \% & 0 \% & 0 \%\end{array}$


T E S T N U M B E R : 8-6

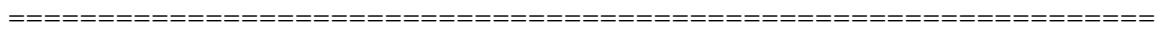

Crack Initiation at Insert:

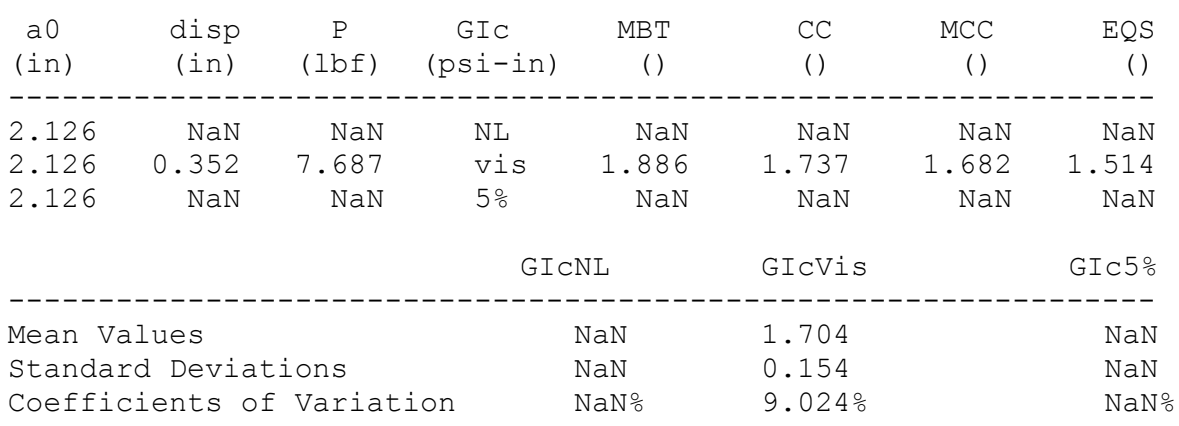

Crack Initiation at Precrack:

\begin{tabular}{|c|c|c|c|c|c|c|c|}
\hline $\begin{array}{c}\mathrm{ap} \\
(\mathrm{in})\end{array}$ & $\begin{array}{l}\text { disp } \\
(\text { in) }\end{array}$ & $\begin{array}{c}\mathrm{P} \\
(1 \mathrm{bf})\end{array}$ & $\begin{array}{c}\text { GIC } \\
(p s i-i n)\end{array}$ & $\begin{array}{c}\text { MBT } \\
()\end{array}$ & $\begin{array}{l}\mathrm{CC} \\
()\end{array}$ & $\begin{array}{c}\mathrm{MCC} \\
()\end{array}$ & $\begin{array}{c}\text { EQS } \\
(\text { ) }\end{array}$ \\
\hline & & & & & & & \\
\hline $\begin{array}{l}3.031 \\
3.031\end{array}$ & $\begin{array}{l}0.964 \\
1.037\end{array}$ & $\begin{array}{l}7.562 \\
8.005\end{array}$ & $\begin{array}{l}\text { NL } \\
\text { vis }\end{array}$ & $\begin{array}{l}3.564 \\
4.060\end{array}$ & $\begin{array}{l}3.282 \\
3.739\end{array}$ & $\begin{array}{l}3.221 \\
3.450\end{array}$ & $\begin{array}{l}2.978 \\
3.155\end{array}$ \\
\hline 3.031 & NaN & $\mathrm{NaN}$ & $5 \%$ & $\mathrm{NaN}$ & $\mathrm{NaN}$ & $\mathrm{NaN}$ & \\
\hline
\end{tabular}

\begin{tabular}{|c|c|c|c|}
\hline & GICNL & GICVis & GIC $5 \%$ \\
\hline 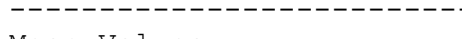 & 3261 & $3 \quad 601$ & NT \\
\hline Mean Values & 3.261 & 3.601 & $\mathrm{NaN}$ \\
\hline Standard Deviations & 0.241 & 0.388 & $\mathrm{NaN}$ \\
\hline Coefficients of Variation & $7.383 \%$ & $10.78 \%$ & $\mathrm{NaN} \%$ \\
\hline
\end{tabular}

Crack Propagation Values:

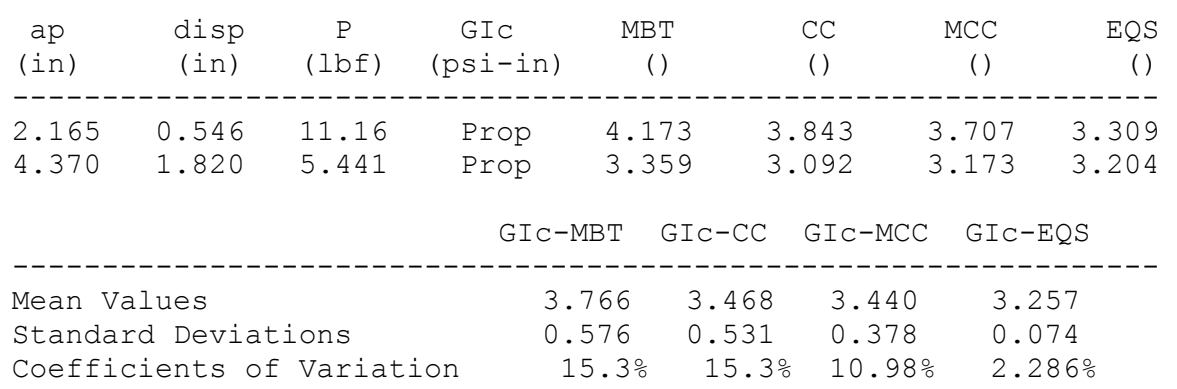


P L A T E

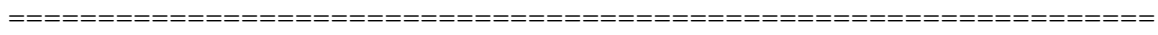

Crack Initiation at Insert

\begin{tabular}{|c|c|c|c|c|c|c|c|}
\hline $\begin{array}{c}\mathrm{a} 0 \\
(\mathrm{in})\end{array}$ & $\begin{array}{l}\text { disp } \\
\text { (in) }\end{array}$ & $\begin{array}{c}\mathrm{P} \\
(1 \mathrm{~b} f)\end{array}$ & $\begin{array}{c}\text { GIC } \\
(\operatorname{psi-in)}\end{array}$ & $\begin{array}{c}\mathrm{MBT} \\
(\mathrm{l}\end{array}$ & $\begin{array}{l}\mathrm{CC} \\
()\end{array}$ & $\begin{array}{c}\mathrm{MCC} \\
(\text { ) }\end{array}$ & $\begin{array}{c}\text { EQS } \\
(\text { ) }\end{array}$ \\
\hline \multicolumn{8}{|c|}{ Mean Values----------------------------------------------------- } \\
\hline 2.110 & 0.421 & 10.36 & $\mathrm{NL}$ & 3.091 & 2.812 & 2.723 & 2.533 \\
\hline 2.110 & 0.402 & 9.624 & vis & 2.791 & 2.574 & 2.497 & 2.302 \\
\hline 2.110 & $\mathrm{NaN}$ & $\mathrm{NaN}$ & $5 \circ$ & $\mathrm{NaN}$ & $\mathrm{NaN}$ & $\mathrm{NaN}$ & $\mathrm{NaN}$ \\
\hline Standard & \multicolumn{7}{|c|}{ Deviations--------------------------------------------- } \\
\hline $1.94 e-02$ & 0.069 & 1.030 & NL & 0.774 & 0.821 & 0.838 & 0.518 \\
\hline 1. $94 e-02$ & 0.057 & 1.180 & vis & 0.674 & 0.643 & 0.640 & 0.55 \\
\hline $1.94 e-02$ & 0.069 & $\mathrm{NaN}$ & $5 \%$ & $\mathrm{NaN}$ & $\mathrm{NaN}$ & $\mathrm{NaN}$ & $\mathrm{NaN}$ \\
\hline \multicolumn{8}{|c|}{ Coefficients of Variation $\left(\frac{\circ}{\circ}\right)------------------------------------$} \\
\hline $9.18 e-01$ & 16.28 & 9.914 & NL & 25.04 & 29.19 & 30.79 & 20.45 \\
\hline $8 e-01$ & 14.19 & 12.23 & vis & 24.16 & 24.96 & 25.65 & 24.07 \\
\hline $18 e-01$ & NaN & $\mathrm{NaN}$ & $5 \%$ & $\mathrm{NaN}$ & $\mathrm{NaN}$ & $\mathrm{NaN}$ & $\mathrm{NaN}$ \\
\hline
\end{tabular}

Crack Initiation at Precrack

\begin{tabular}{|c|c|c|c|c|c|c|c|}
\hline $\begin{array}{l}\operatorname{ap} \\
(i n)\end{array}$ & $\begin{array}{c}\text { disp } \\
(i n)\end{array}$ & $\begin{array}{c}\mathrm{P} \\
(1 \mathrm{bf})\end{array}$ & $\begin{array}{c}\text { GIC } \\
(p s i-i n)\end{array}$ & $\begin{array}{c}\mathrm{MBT} \\
()\end{array}$ & $\begin{array}{l}\mathrm{CC} \\
()\end{array}$ & $\begin{array}{c}\mathrm{MCC} \\
()\end{array}$ & $\begin{array}{r}\text { EQS } \\
()\end{array}$ \\
\hline \multicolumn{8}{|c|}{ Mean Values----------------------------------------------------- } \\
\hline 2.907 & 0.875 & 8.444 & NL & 3.852 & 3.579 & 3.526 & 3.298 \\
\hline 2.907 & 0.928 & 8.86 & vis & 4.239 & 3.9 & 3.644 & \\
\hline 2.907 & NaN & NaN & $5 \%$ & NaN & $\mathrm{NaN}$ & NaN & $\mathrm{Nal}$ \\
\hline Standard & Deviati & $1 S-----$ & & & ------1 & ------- & ----- \\
\hline $2.07 e-01$ & 0.147 & 0.572 & NL & 0.476 & 0.385 & 0.383 & \\
\hline $2.07 e-01$ & 0.149 & 0.537 & vis & 0.497 & 0.4013 & 0.355 & \\
\hline $2.07 e-01$ & 0.147 & NaN & $5 \%$ & $\mathrm{NaN}$ & $\mathrm{NaN}$ & $\mathrm{NaN}$ & \\
\hline \multicolumn{8}{|c|}{ Coefficients of Variation $\left(\frac{\circ}{0}\right)------------------------------------$} \\
\hline $7.14 e+00$ & 16.82 & 6.773 & NL & 12.37 & 10.76 & 10.86 & 17.03 \\
\hline $7.14 e+00$ & 16.03 & 6.059 & vis & 11.73 & 10.29 & 9.734 & 15 . \\
\hline $7.14 e+00$ & NaN & NaN & $5 \%$ & NaN & $\mathrm{NaN}$ & NaN & \\
\hline
\end{tabular}

Crack Propagation Values

\begin{tabular}{|c|c|c|c|c|}
\hline------------------------- & $\begin{array}{l}\text { GIC-MBT } \\
---------\end{array}$ & $\begin{array}{l}\mathrm{GIC}-\mathrm{CC} \\
--------\end{array}$ & $\begin{array}{l}\text { GIC }-\mathrm{MCC} \\
--------\end{array}$ & $\begin{array}{l}\mathrm{GIC}-\mathrm{EQS} \\
-----------\end{array}$ \\
\hline Mean Values & 3.986 & 3.669 & 3.673 & 3.634 \\
\hline Standard Deviations & 0.261 & 0.238 & 0.128 & 0.176 \\
\hline Coefficients of Variation & $6.673 \%$ & $6.673 \%$ & $3.714 \%$ & $4.602 \%$ \\
\hline
\end{tabular}

Slopes and Intercepts

\begin{tabular}{|c|c|c|c|}
\hline & lta & $\mathrm{n}$ & A1 \\
\hline Mean Values & 0 & 2.765 & 52.58 \\
\hline Standard Deviations & 0 & 0.080 & 2.55 \\
\hline Coefficients of Variation & $\mathrm{NaN} \div$ & $2.896 \%$ & $4.843 \%$ \\
\hline
\end{tabular}




\section{H.4 Plate}

T E S T $N$ U $M$ B E R :

$9-1$

Crack Initiation at Insert:

\begin{tabular}{|c|c|c|c|c|c|c|c|}
\hline $\begin{array}{c}\mathrm{a} 0 \\
(\mathrm{in})\end{array}$ & $\begin{array}{l}\text { disp } \\
\text { (in) }\end{array}$ & $\begin{array}{c}P \\
(1 \mathrm{bf})\end{array}$ & $\begin{array}{c}\text { GIC } \\
(p s i-i n)\end{array}$ & $\begin{array}{c}\mathrm{MBT} \\
()\end{array}$ & $\begin{array}{l}\mathrm{CC} \\
()\end{array}$ & $\begin{array}{c}\mathrm{MCC} \\
()\end{array}$ & \\
\hline & 0.369 & 11.28 & NL & 3.057 & 2.680 & 2.570 & \\
\hline 68 & 0.374 & 11.38 & vis & 3.127 & 2.742 & 2.625 & 2.2 \\
\hline 968 & $\mathrm{NaN}$ & $\mathrm{NaN}$ & $5 \%$ & $\mathrm{NaN}$ & $\mathrm{NaN}$ & $\mathrm{NaN}$ & \\
\hline
\end{tabular}

\begin{tabular}{|c|c|c|c|}
\hline & GICNL & GICVis & GIC $5 \%$ \\
\hline ean Values & 2.617 & 2.674 & $\mathrm{NaN}$ \\
\hline tandard Deviations & 0.368 & 0.381 & $\mathrm{NaN}$ \\
\hline Coefficients of Variation & $14.05 \%$ & $14.25 \%$ & $\mathrm{NaN} \circ$ \\
\hline
\end{tabular}

Crack Initiation at Precrack:

\begin{tabular}{|c|c|c|c|c|c|c|c|}
\hline $\begin{array}{l}\mathrm{ap} \\
(\mathrm{in})\end{array}$ & $\begin{array}{l}\text { disp } \\
(i n)\end{array}$ & $\begin{array}{c}\mathrm{P} \\
(1 \mathrm{bf})\end{array}$ & $\begin{array}{c}\text { GIC } \\
(p s i-i n)\end{array}$ & $\begin{array}{c}\mathrm{MBT} \\
()\end{array}$ & $\begin{array}{l}\mathrm{CC} \\
()\end{array}$ & $\begin{array}{c}\mathrm{MCC} \\
()\end{array}$ & \\
\hline 32 & 0.899 & 17.68 & $\mathrm{NL}$ & 9.420 & 8.259 & 8.069 & \\
\hline 2.323 & 1.000 & 19.22 & vis & 12.30 & 10.78 & 9.536 & \\
\hline 2.323 & NaN & $\mathrm{NaN}$ & $5 \%$ & $\mathrm{NaN}$ & NaN & $\mathrm{NaN}$ & \\
\hline
\end{tabular}

\begin{tabular}{|c|c|c|c|}
\hline & GICNL & GICVis & GIC5\% \\
\hline Mea & 8190 & 1020 & \\
\hline Standard Deviations & 0.190 & 1.750 & Nan \\
\hline Coefficients of Variation & $11.88 \%$ & $17.13 \%$ & \\
\hline
\end{tabular}

Crack Propagation Values:

\begin{tabular}{|c|c|c|c|c|c|c|c|}
\hline $\begin{array}{c}a p \\
(i n)\end{array}$ & $\begin{array}{l}\text { disp } \\
(\text { in) }\end{array}$ & $\begin{array}{c}\mathrm{P} \\
(1 \mathrm{bf})\end{array}$ & $\begin{array}{c}\text { GIC } \\
(p s i-i n)\end{array}$ & $\begin{array}{c}\mathrm{MBT} \\
(\text { ) }\end{array}$ & $\begin{array}{l}\mathrm{CC} \\
()\end{array}$ & $\begin{array}{c}\mathrm{MCC} \\
()\end{array}$ & $\begin{array}{c}\text { EQS } \\
()\end{array}$ \\
\hline \multicolumn{8}{|c|}{ 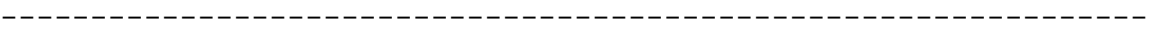 } \\
\hline 2.087 & 0.637 & 16.04 & Prop & 6.890 & 6.041 & 5.758 & 4.785 \\
\hline 2.106 & 0.773 & 18.61 & Prop & 9.436 & 8.273 & 7.840 & 6.440 \\
\hline 2.402 & 1.071 & 19.64 & Prop & 11.82 & 10.36 & 10.23 & 9.111 \\
\hline 2.874 & 1.439 & 16.05 & Prop & 10.68 & 9.360 & 9.368 & 8.582 \\
\hline 2.913 & 1.497 & 16.22 & Prop & 11.02 & 9.664 & 9.711 & 8.966 \\
\hline 3.406 & 1.915 & 13.26 & Prop & 9.710 & 8.513 & 8.611 & 8.057 \\
\hline 3.425 & 1.972 & 13.33 & Prop & 9.935 & 8.711 & 8.793 & 8.194 \\
\hline 3.465 & 1.991 & 13.01 & Prop & 9.692 & 8.498 & 8.579 & 7.995 \\
\hline 3.504 & 2.249 & 14.41 & Prop & 11.61 & 10.18 & 10.32 & 9.713 \\
\hline 3.622 & 2.251 & 14.24 & Prop & 11.23 & 9.849 & 10.28 & 10.25 \\
\hline 4.095 & 2.725 & 11.94 & Prop & 9.907 & 8.686 & 9.070 & 9.046 \\
\hline 4.114 & 3.057 & 13.06 & Prop & 11.58 & 10.16 & 10.57 & 10.46 \\
\hline 4.134 & 3.065 & 12.99 & Prop & 11.51 & 10.09 & 10.52 & 10.46 \\
\hline
\end{tabular}

\begin{tabular}{|c|c|c|c|c|}
\hline & GIC-MBT & $\mathrm{GIC}-\mathrm{CC}$ & GIC-MCC & GIC-EQS \\
\hline Mean Values & 10.39 & 9.106 & 9.203 & 8.620 \\
\hline Standard Deviations & 1.350 & 1.190 & 1.350 & 1.620 \\
\hline Coefficients of Variation & $13.02 \%$ & $13.02 \%$ & $14.71 \%$ & $18.80 \%$ \\
\hline
\end{tabular}


T E S T N U M B E R :

$9-2$

Crack Initiation at Insert:

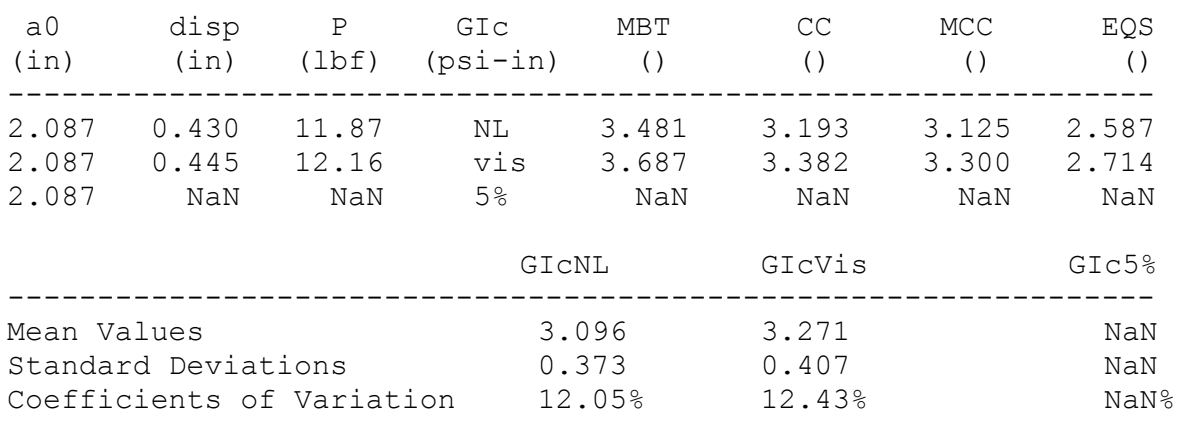

Crack Initiation at Precrack:

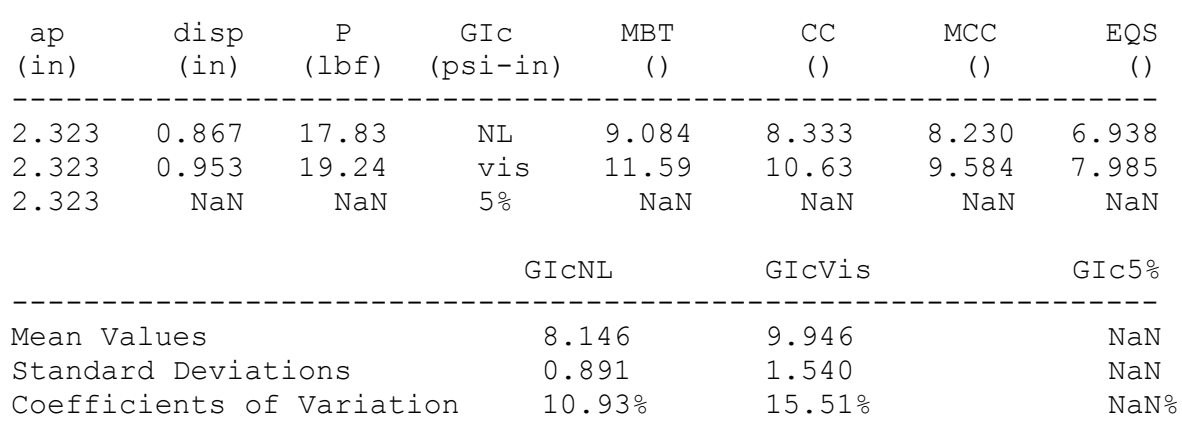

Crack Propagation Values:

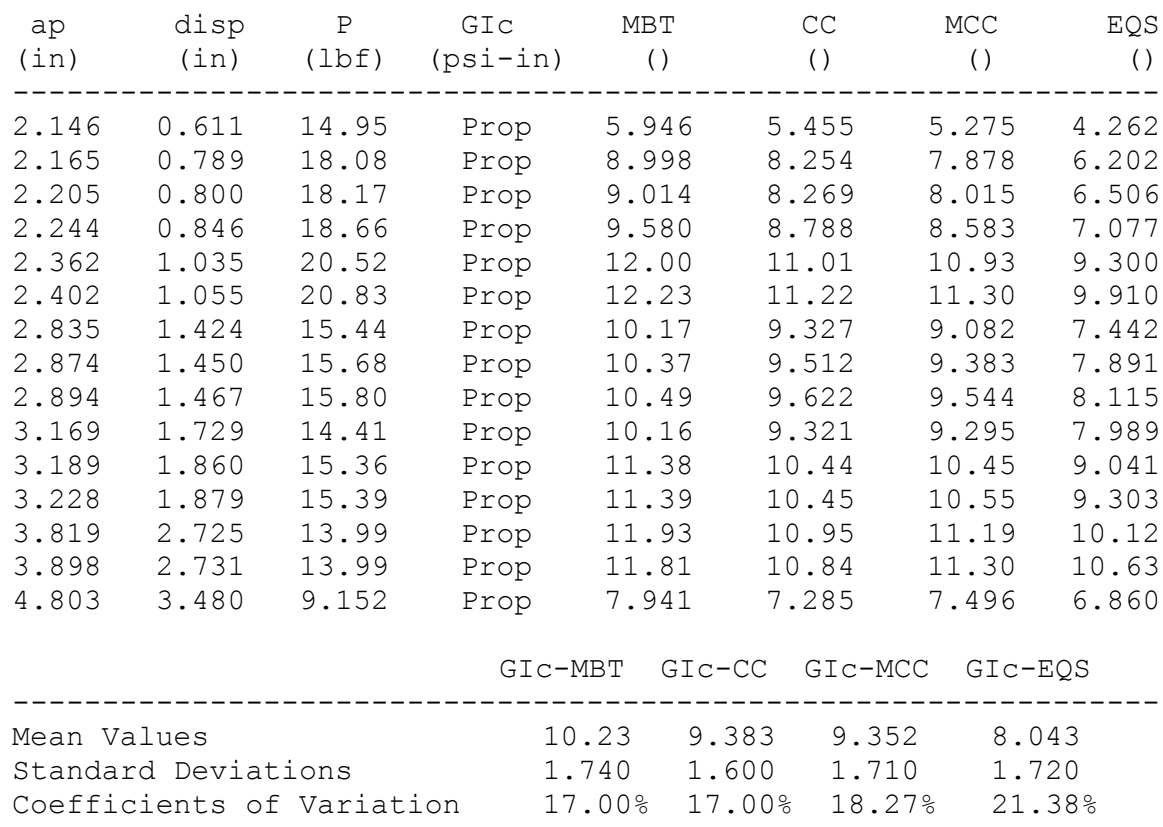


T E S T N U M B E R :

$9-3$

Crack Initiation at Insert:

\begin{tabular}{|c|c|c|c|c|c|c|c|}
\hline $\begin{array}{c}\mathrm{a} 0 \\
(\mathrm{in})\end{array}$ & $\begin{array}{l}\text { disp } \\
(\text { in) }\end{array}$ & $\begin{array}{c}\mathrm{P} \\
(1 \mathrm{bf})\end{array}$ & $\begin{array}{c}\text { GIC } \\
(p s i-i n)\end{array}$ & $\begin{array}{c}\mathrm{MBT} \\
()\end{array}$ & $\begin{array}{l}\mathrm{CC} \\
()\end{array}$ & $\begin{array}{c}\mathrm{MCC} \\
()\end{array}$ & $\begin{array}{c}\text { EQS } \\
()\end{array}$ \\
\hline---- & & ----- & 10 & --- & ------- & ----- & ----- \\
\hline 2.008 & 0.392 & 12.96 & $\mathrm{NL}$ & 3.639 & 3.452 & 3.452 & 2.894 \\
\hline 2.008 & 0.410 & 13.21 & vis & 3.876 & 3.677 & 3.645 & 3.001 \\
\hline 2.008 & $\mathrm{NaN}$ & $\mathrm{NaN}$ & $5 \%$ & $\mathrm{NaN}$ & $\mathrm{NaN}$ & $\mathrm{NaN}$ & $\mathrm{NaN}$ \\
\hline \multicolumn{5}{|c|}{ GICNL } & GICVis & & GIC 5\% \\
\hline \multicolumn{5}{|c|}{ Mean Values } & 3.550 & & \\
\hline \multicolumn{3}{|c|}{ Standard Deviations } & & 22 & 0.380 & & $\mathrm{aN}$ \\
\hline Coeffi & ents & Variat & on & $96 \%$ & $10.7 \%$ & & $\mathrm{aN} \div$ \\
\hline
\end{tabular}

Crack Initiation at Precrack:

\begin{tabular}{|c|c|c|c|c|c|c|c|}
\hline $\begin{array}{l}\mathrm{ap} \\
(\mathrm{in})\end{array}$ & $\begin{array}{c}\text { disp } \\
\text { (in) }\end{array}$ & $\begin{array}{c}\mathrm{P} \\
(1 \mathrm{bf})\end{array}$ & $\begin{array}{c}\text { GIC } \\
(p s i-i n)\end{array}$ & $\begin{array}{c}\text { MBT } \\
()\end{array}$ & $\begin{array}{l}\mathrm{CC} \\
()\end{array}$ & $\begin{array}{c}\mathrm{MCC} \\
()\end{array}$ & $\begin{array}{c}\text { EQS } \\
()\end{array}$ \\
\hline \multicolumn{8}{|c|}{ 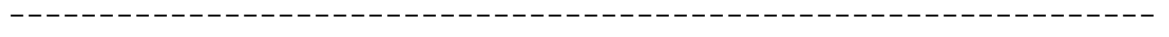 } \\
\hline 2.461 & $\mathrm{NaN}$ & $\mathrm{NaN}$ & NL & $\mathrm{NaN}$ & $\mathrm{NaN}$ & $\mathrm{NaN}$ & $\mathrm{NaN}$ \\
\hline 2.461 & 1.150 & 21.72 & vis & 15.04 & 14.27 & 13.07 & 11.32 \\
\hline \multirow[t]{2}{*}{2.461} & $\mathrm{NaN}$ & $\mathrm{NaN}$ & $5 \%$ & $\mathrm{NaN}$ & $\mathrm{NaN}$ & $\mathrm{NaN}$ & $\mathrm{NaN}$ \\
\hline & & & \multicolumn{2}{|c|}{ GICNL } & GICVis & & GIC $5 \%$ \\
\hline \multirow{2}{*}{\multicolumn{5}{|c|}{ Mean Values }} & & & \\
\hline \multirow{2}{*}{\multicolumn{3}{|c|}{ Standard Deviations }} & & $\mathrm{Nan}$ & $\begin{array}{ll}13.42 \\
1\end{array}$ & & \\
\hline & & & Coefficients of Variation & $\begin{array}{l}\mathrm{NaN} \\
\mathrm{NaN} \%\end{array}$ & $\begin{array}{l}1.620 \\
12.09 \%\end{array}$ & & \\
\hline
\end{tabular}

Crack Propagation Values:

\begin{tabular}{|c|c|c|c|c|c|c|c|}
\hline $\begin{array}{l}\mathrm{ap} \\
(\mathrm{in})\end{array}$ & $\begin{array}{l}\text { disp } \\
(i n)\end{array}$ & $\begin{array}{c}\mathrm{P} \\
(1 \mathrm{bf})\end{array}$ & $\begin{array}{c}\text { GIC } \\
(\mathrm{psi-in)}\end{array}$ & $\begin{array}{c}\mathrm{MBT} \\
()\end{array}$ & $\begin{array}{l}\mathrm{CC} \\
()\end{array}$ & $\begin{array}{c}\mathrm{MCC} \\
()\end{array}$ & $\begin{array}{r}E Q S \\
()\end{array}$ \\
\hline \multicolumn{8}{|l|}{------ } \\
\hline 2.067 & 0.602 & 16.95 & Prop & 6.952 & 6.596 & 6.431 & 5.125 \\
\hline 2.106 & 0.663 & 18.39 & Prop & 8.097 & 7.683 & 7.597 & 6.228 \\
\hline 2.146 & 0.688 & 18.03 & Prop & 8.077 & 7.664 & 7.575 & 6.205 \\
\hline 2.165 & 0.744 & 19.07 & Prop & 9.100 & 8.634 & 8.549 & 7.028 \\
\hline 2.185 & 0.825 & 20.44 & Prop & 10.60 & 10.06 & 9.938 & 8.135 \\
\hline 3.071 & 1.354 & 13.02 & Prop & 7.795 & 7.396 & 7.490 & 6.440 \\
\hline 3.11 & 1.575 & 14.31 & Prop & 9.607 & 9.115 & 9.176 & 7.796 \\
\hline 3.15 & 1.639 & 14.87 & Prop & 10.20 & 9.680 & 9.864 & 8.586 \\
\hline 3.189 & 1.724 & 15.24 & Prop & 10.78 & 10.23 & 10.46 & 9.169 \\
\hline 3.563 & 1.908 & 11.3 & Prop & 7.961 & 7.553 & 7.552 & 6.329 \\
\hline 3.622 & 2.247 & 13.11 & Prop & 10.30 & 9.773 & 9.885 & 8.479 \\
\hline 3.661 & 2.316 & 13.43 & Prop & 10.70 & 10.15 & 10.36 & 9.03 \\
\hline 3.701 & 2.320 & 13.41 & Prop & 10.62 & 10.07 & 10.38 & 9.23 \\
\hline 4.055 & 2.824 & 11.87 & Prop & 10.10 & 9.580 & 9.724 & 8.398 \\
\hline
\end{tabular}

\begin{tabular}{|c|c|c|c|c|}
\hline & GIC-MBT & $\mathrm{GIC}-\mathrm{CC}$ & GIC-MCC & GIC-EQS \\
\hline & & & & \\
\hline ean Values & & 8.870 & 8.927 & 7.585 \\
\hline tandard Deviations & 1.320 & 1.250 & 1.360 & 1.330 \\
\hline Coefficients of Variation & $14.11 \%$ & $14.11 \%$ & $15.2 \%$ & $17.59 \%$ \\
\hline
\end{tabular}


T E S T $\mathrm{N}$ U $\mathrm{M}$ B E $\mathrm{R}$ :

$9-4$

Crack Initiation at Insert:

\begin{tabular}{|c|c|c|c|c|c|c|c|}
\hline $\begin{array}{c}\mathrm{a} 0 \\
(\mathrm{in})\end{array}$ & $\begin{array}{l}\text { disp } \\
(\text { in) }\end{array}$ & $\begin{array}{c}\mathrm{P} \\
(1 \mathrm{bf})\end{array}$ & $\begin{array}{c}\text { GIC } \\
(p s i-i n)\end{array}$ & $\begin{array}{c}\mathrm{MBT} \\
()\end{array}$ & $\begin{array}{l}\mathrm{CC} \\
()\end{array}$ & $\begin{array}{c}\mathrm{MCC} \\
()\end{array}$ & $\begin{array}{c}\text { EQS } \\
()\end{array}$ \\
\hline---- & & ----- & -------- & 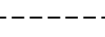 & -------1 & ----- & ----- \\
\hline 2.087 & 0.442 & 12.65 & $\mathrm{NL}$ & 3.804 & 3.742 & 3.927 & 2.917 \\
\hline 2.087 & 0.470 & 13.30 & vis & 4.235 & 4.166 & 4.359 & 3.218 \\
\hline 2.087 & $\mathrm{NaN}$ & $\mathrm{NaN}$ & $5 \div$ & $\mathrm{NaN}$ & $\mathrm{NaN}$ & $\mathrm{NaN}$ & $\mathrm{NaN}$ \\
\hline \multicolumn{5}{|c|}{ GICNL } & GICVis & & GIC 5\% \\
\hline \multicolumn{5}{|c|}{ Mean Values } & 3.994 & & \\
\hline \multicolumn{3}{|c|}{ Standard Deviations } & & 60 & 0.524 & & $\mathrm{NaN}$ \\
\hline Coeffi & ents o & Variat & on & $79 \%$ & $13.11 \%$ & & $\mathrm{NaN} \%$ \\
\hline
\end{tabular}

Crack Initiation at Precrack:

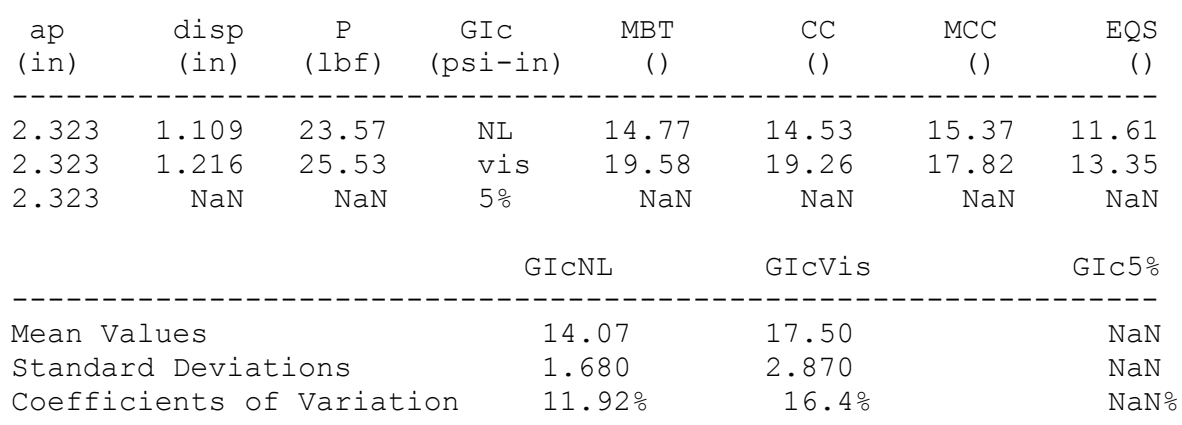

Crack Propagation Values:

\begin{tabular}{|c|c|c|c|c|c|c|c|}
\hline $\begin{array}{c}\text { ap } \\
(i n)\end{array}$ & $\begin{array}{l}\text { disp } \\
\text { (in) }\end{array}$ & $\begin{array}{c}\mathrm{P} \\
(1 \mathrm{bf})\end{array}$ & $\begin{array}{c}\text { GIC } \\
(\mathrm{psi}-i n)\end{array}$ & $\begin{array}{c}\mathrm{MBT} \\
()\end{array}$ & $\begin{array}{l}\mathrm{CC} \\
()\end{array}$ & $\begin{array}{c}\mathrm{MCC} \\
()\end{array}$ & $\begin{array}{c}E Q S \\
()\end{array}$ \\
\hline 2.165 & 0.674 & 16.93 & Prop & 7.296 & 7.178 & 7.487 & 5.495 \\
\hline 2.205 & 0.731 & 17.93 & Prop & 8.189 & 8.057 & 8.489 & 6.35 \\
\hline 3.307 & 1.902 & 13.99 & Prop & 10.26 & 10.09 & 10.67 & 8.05 \\
\hline 3.661 & 2.260 & 13.45 & Prop & 10.41 & 10.24 & 11.18 & 8.98 \\
\hline 3.681 & 2.446 & 14.46 & Prop & 11.76 & 11.57 & 12.67 & 10.2 \\
\hline 3.74 & 2.508 & 14.61 & Prop & 11.96 & 11.76 & 13.02 & 10.7 \\
\hline 4.665 & 3.637 & 11.21 & Prop & 10.09 & 9.926 & 11.08 & 9.32 \\
\hline 4.705 & 3.649 & 7.074 & Prop & 6.353 & 6.250 & 6.030 & 3.786 \\
\hline
\end{tabular}

\begin{tabular}{|c|c|c|c|c|}
\hline & GIC-MBT & $\mathrm{IC}-\mathrm{CC}$ & GIC-MCC & GIC-EQS \\
\hline $\mathrm{Va}$ & 9540 & 9385 & $1 \cap 08$ & 7874 \\
\hline Standard Deviations & 2.050 & 2.020 & 2.490 & 2450 \\
\hline Coefficients of Variation & $21.47 \%$ & $21.47 \%$ & $24.75 \%$ & $31.08 \%$ \\
\hline
\end{tabular}


T E S T N U M B E R :

$9-5$

Crack Initiation at Insert:

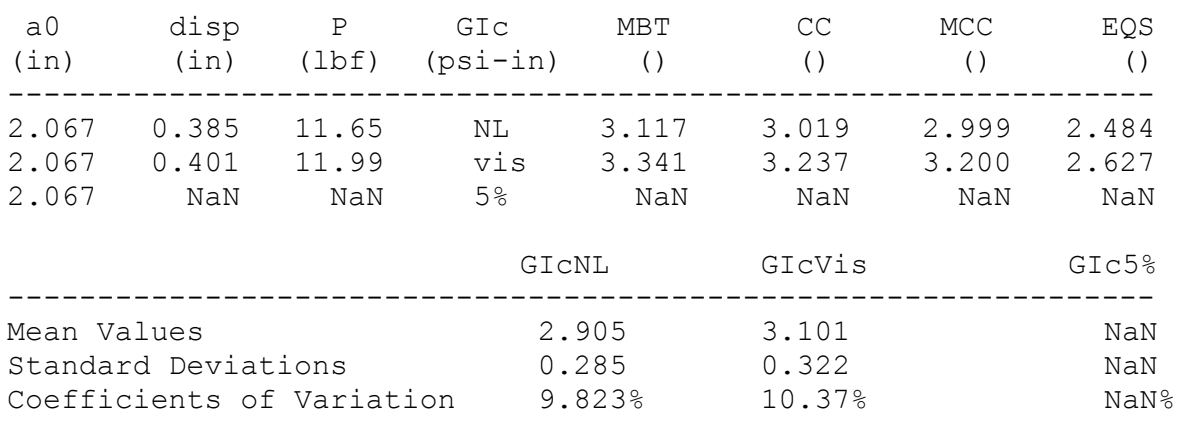

Crack Initiation at Precrack:

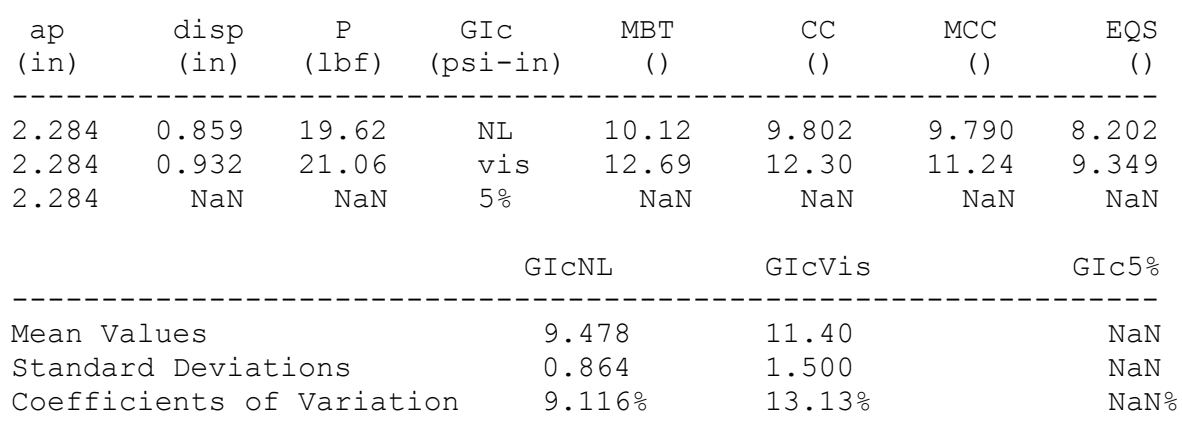

Crack Propagation Values:

\begin{tabular}{|c|c|c|c|c|c|c|c|}
\hline $\begin{array}{l}\mathrm{ap} \\
(\mathrm{in})\end{array}$ & $\begin{array}{l}\text { disp } \\
(\text { in) }\end{array}$ & $\begin{array}{c}P \\
(1 \mathrm{bf})\end{array}$ & $\begin{array}{c}\text { GIC } \\
(p s i-i n)\end{array}$ & $\begin{array}{c}\mathrm{MBT} \\
()\end{array}$ & $\begin{array}{l}\mathrm{CC} \\
()\end{array}$ & $\begin{array}{c}\mathrm{MCC} \\
()\end{array}$ & $\begin{array}{r}E Q S \\
()\end{array}$ \\
\hline----- & ------ & ----- & 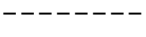 & ----- & ----- & ----- & ----- \\
\hline 2.106 & 0.554 & 14.94 & Prop & 5.558 & 5.384 & 5.243 & 4.175 \\
\hline 2.146 & 0.593 & 15.79 & Prop & 6.149 & 5.957 & 5.886 & 4.825 \\
\hline 2.244 & 0.736 & 17.54 & Prop & 7.999 & 7.749 & 7.715 & 6.422 \\
\hline 2.776 & 1.221 & 16.29 & Prop & 9.673 & 9.371 & 9.510 & 8.226 \\
\hline 3.110 & 1.613 & 14.41 & Prop & 9.819 & 9.512 & 9.465 & 7.870 \\
\hline 3.484 & 1.970 & 12.29 & Prop & 8.978 & 8.697 & 8.601 & 7.064 \\
\hline 3.524 & 2.007 & 12.45 & Prop & 9.153 & 8.867 & 8.852 & 7.408 \\
\hline 3.543 & 2.099 & 12.75 & Prop & 9.649 & 9.348 & 9.318 & 7.775 \\
\hline 3.583 & 2.290 & 13.70 & Prop & 10.94 & 10.60 & 10.63 & 8.976 \\
\hline 4.311 & 2.837 & 10.03 & Prop & 8.219 & 7.962 & 8.064 & 6.946 \\
\hline 4.331 & 3.138 & 11.21 & Prop & 9.749 & 9.444 & 9.640 & 8.434 \\
\hline
\end{tabular}

\begin{tabular}{|c|c|c|c|c|}
\hline & GIC-MBT & $\mathrm{GIC}-\mathrm{CC}$ & GIC-MCC & GIC-EQS \\
\hline Mean Values & 8.717 & 8.444 & 8.447 & 7.102 \\
\hline Standard Deviations & 1.630 & 1.580 & 1.640 & 1.480 \\
\hline Coefficients of Variation & $18.71 \%$ & $18.71 \%$ & $19.38 \%$ & $20.85 \%$ \\
\hline
\end{tabular}


P L A T E S T A T I S T I C S : P L A T E 9

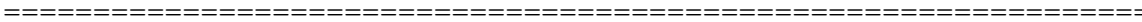

Crack Initiation at Insert

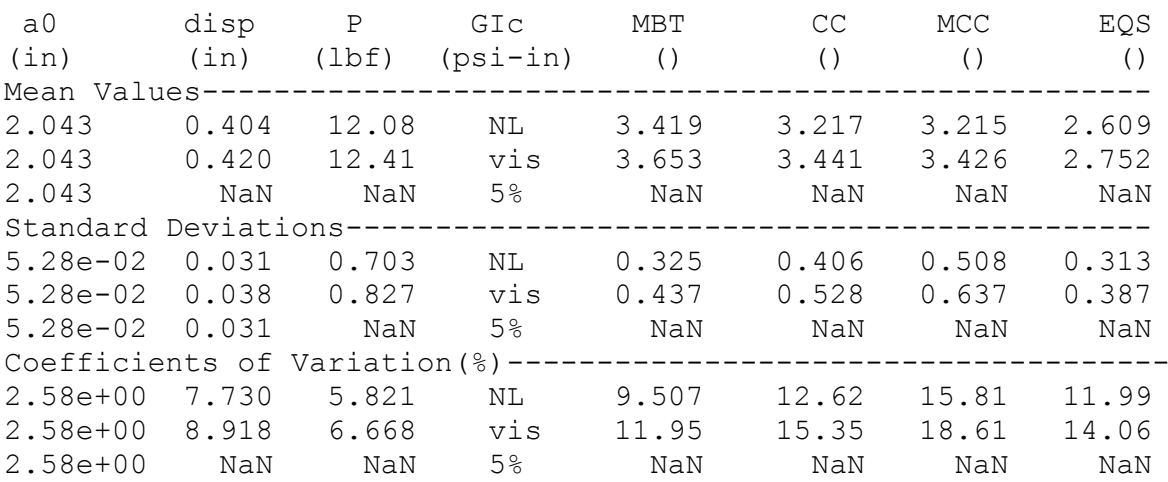

Crack Initiation at Precrack

\begin{tabular}{|c|c|c|c|c|c|c|c|}
\hline $\begin{array}{l}\operatorname{ap} \\
(\mathrm{in})\end{array}$ & $\begin{array}{l}\text { disp } \\
(\text { in) }\end{array}$ & $\begin{array}{c}P \\
l b f)\end{array}$ & $\begin{array}{c}\text { GIC } \\
(p s i-i n)\end{array}$ & $\begin{array}{c}\text { MBT } \\
()\end{array}$ & $\begin{array}{l}\mathrm{CC} \\
()\end{array}$ & $\begin{array}{c}\mathrm{MCC} \\
()\end{array}$ & $\begin{array}{c}\text { EQS } \\
()\end{array}$ \\
\hline \multicolumn{8}{|c|}{ Mean Values------------------------------------------------------ } \\
\hline 2.343 & 0.934 & 19.67 & NL & 10.85 & 10.23 & 10.37 & 8.449 \\
\hline 2.343 & 1.050 & 21.35 & vis & 14.24 & 13.45 & 12.25 & 10.04 \\
\hline 2.343 & $\mathrm{NaN}$ & $\mathrm{NaN}$ & $5 \%$ & $\mathrm{NaN}$ & $\mathrm{NaN}$ & $\mathrm{NaN}$ & NaN \\
\hline Standard & \multicolumn{7}{|c|}{ Deviations ---------------------------------------------} \\
\hline $2 e-02$ & 0.118 & 2.745 & NL & 2.648 & 2.951 & 3.427 & \\
\hline $6.82 e-02$ & 0.126 & 2.582 & vis & 3.254 & 3.565 & 3.436 & 2.274 \\
\hline $6.82 e-02$ & 0.118 & $\mathrm{NaN}$ & $5 \%$ & $\mathrm{NaN}$ & $\mathrm{NaN}$ & $\mathrm{NaN}$ & NaN \\
\hline \multicolumn{8}{|c|}{ Coefficients of Variation $\left(\frac{\circ}{\circ}\right)-------------------------------------$} \\
\hline $2.91 e+00$ & 12.67 & 13.95 & NL & 24.41 & 28.85 & 33.06 & 25.85 \\
\hline $2.91 e+00$ & 11.98 & 12.09 & vis & 22.85 & 26.51 & 28.05 & 22.65 \\
\hline $2.91 e+00$ & $\mathrm{NaN}$ & NaN & $5 \%$ & $\mathrm{NaN}$ & $\mathrm{NaN}$ & $\mathrm{NaN}$ & $\mathrm{NaN}$ \\
\hline
\end{tabular}

Crack Propagation Values

\begin{tabular}{|c|c|c|c|c|}
\hline & GIC-MBT & $\mathrm{GIC}-\mathrm{CC}$ & GIC-MCC & GIC-EQS \\
\hline Mean Values & 9.644 & 9.038 & 9.202 & 7.845 \\
\hline Standard Deviations & 1.620 & 1.530 & 1.710 & 1.720 \\
\hline Coefficients of Variation & $16.86 \%$ & $16.86 \%$ & $18.46 \%$ & $21.94 \%$ \\
\hline
\end{tabular}

Slopes and Intercepts

\begin{tabular}{|c|c|c|c|}
\hline & Delta & $\mathrm{n}$ & A1 \\
\hline Mean Values & $-3.482 e-017$ & 2.817 & 49.30 \\
\hline Standard Deviations & $7.79 e-017$ & 0.128 & 3.130 \\
\hline Coefficients of Variation & $-223.6 \%$ & $4.558 \%$ & $6.356 \%$ \\
\hline
\end{tabular}




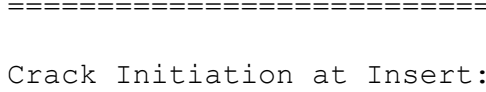

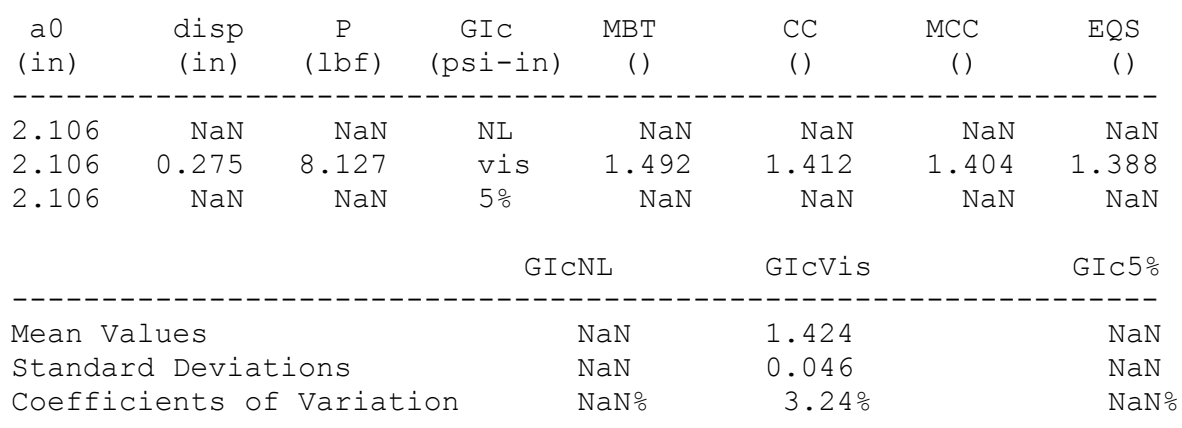

Crack Initiation at Precrack:

\begin{tabular}{|c|c|c|c|c|c|c|c|}
\hline $\begin{array}{l}\mathrm{ap} \\
(\mathrm{in})\end{array}$ & $\begin{array}{c}\text { disp } \\
(i n)\end{array}$ & $\begin{array}{c}P \\
(1 \mathrm{bf})\end{array}$ & $\begin{array}{c}\text { GIC } \\
(p s i-i n)\end{array}$ & $\begin{array}{r}\text { MBT } \\
(\text { ) }\end{array}$ & $\begin{array}{l}\mathrm{CC} \\
()\end{array}$ & $\begin{array}{c}\mathrm{MCC} \\
()\end{array}$ & $\begin{array}{c}\text { EQS } \\
()\end{array}$ \\
\hline \multicolumn{8}{|c|}{ 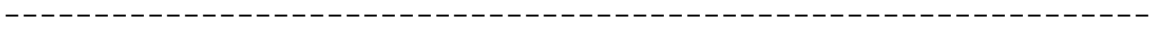 } \\
\hline 3.071 & 1.301 & 13.38 & NL & 7.482 & 7.084 & 7.218 & 7.495 \\
\hline 3.071 & 1.443 & 14.26 & vis & 9.589 & 9.078 & 8.283 & 8.378 \\
\hline 3.071 & $\mathrm{NaN}$ & $\mathrm{NaN}$ & $5 \%$ & $\mathrm{NaN}$ & $\mathrm{NaN}$ & $\mathrm{NaN}$ & $\mathrm{NaN}$ \\
\hline \multicolumn{7}{|c|}{ GICNL } & GIC $5 \%$ \\
\hline \multicolumn{5}{|c|}{ Mean Values } & 8832 & & \\
\hline \multicolumn{3}{|c|}{ Standard Deviations } & & 03 & 0.617 & & $\mathrm{NaN}$ \\
\hline Coeff & ients & Varia & on & $69 \%$ & $6.982 \%$ & & $\mathrm{NaN} \circ$ \\
\hline
\end{tabular}

Crack Propagation Values:

\begin{tabular}{|c|c|c|c|c|c|c|c|}
\hline $\begin{array}{l}\mathrm{ap} \\
(\mathrm{in})\end{array}$ & $\begin{array}{c}\text { disp } \\
(i n)\end{array}$ & $\begin{array}{c}\mathrm{P} \\
(1 \mathrm{bf})\end{array}$ & $\begin{array}{c}\text { GIC } \\
(p s i-i n)\end{array}$ & $\begin{array}{r}\mathrm{MBT} \\
()\end{array}$ & $\begin{array}{l}\text { CC } \\
()\end{array}$ & $\begin{array}{c}\mathrm{MCC} \\
()\end{array}$ & $\begin{array}{r}\text { EQS } \\
(\text { ) }\end{array}$ \\
\hline \multicolumn{8}{|c|}{ 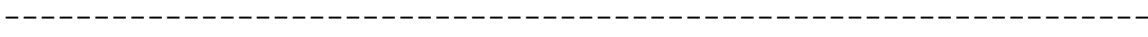 } \\
\hline 2.146 & 0.631 & 15.92 & Prop & 6.366 & 6.027 & 5.790 & 5.342 \\
\hline 2.224 & 0.709 & 17.32 & Prop & 7.465 & 7.067 & 6.959 & 6.748 \\
\hline 2.244 & 0.861 & 20.00 & Prop & 10.17 & 9.626 & 9.407 & 8.985 \\
\hline 2.284 & 0.975 & 22.06 & Prop & 12.30 & 11.65 & 11.48 & 11.15 \\
\hline 2.323 & 1.011 & 22.08 & Prop & 12.52 & 11.85 & 11.75 & 11.53 \\
\hline 3.110 & 1.543 & 15.09 & Prop & 9.629 & 9.116 & 9.253 & 9.534 \\
\hline 3.701 & 2.130 & 11.84 & Prop & 8.523 & 8.069 & 8.073 & 8.082 \\
\hline 3.740 & 2.243 & 12.28 & Prop & 9.113 & 8.628 & 8.679 & 8.783 \\
\hline 3.780 & 2.330 & 12.77 & Prop & 9.666 & 9.151 & 9.305 & 9.622 \\
\hline 3.819 & 2.390 & 12.92 & Prop & 9.881 & 9.355 & 9.568 & 10.01 \\
\hline 4.468 & 2.491 & 7.931 & Prop & 5.602 & 5.303 & 5.320 & 5.353 \\
\hline 4.508 & 2.903 & 9.448 & Prop & 7.409 & 7.014 & 7.150 & 7.431 \\
\hline 4.528 & 2.987 & 9.692 & Prop & 7.726 & 7.314 & 7.481 & 7.827 \\
\hline 4.567 & 3.008 & 9.741 & Prop & 7.758 & 7.345 & 7.573 & 8.051 \\
\hline
\end{tabular}

\begin{tabular}{|c|c|c|c|c|}
\hline & GIC-MBT & $\mathrm{SIC}-\mathrm{CC}$ & $\mathrm{GIC}-\mathrm{MCC}$ & GIC-EQS \\
\hline Mean Values & 8.866 & 8.394 & 8.413 & 8.46 \\
\hline Standard Deviations & 2.020 & 1.910 & 1.890 & 1.880 \\
\hline Coefficients of Variation & $22.73 \%$ & $22.73 \%$ & $22.42 \%$ & $22.25 \%$ \\
\hline
\end{tabular}


T E S T N U M B E R :

$10-2$

Crack Initiation at Insert:

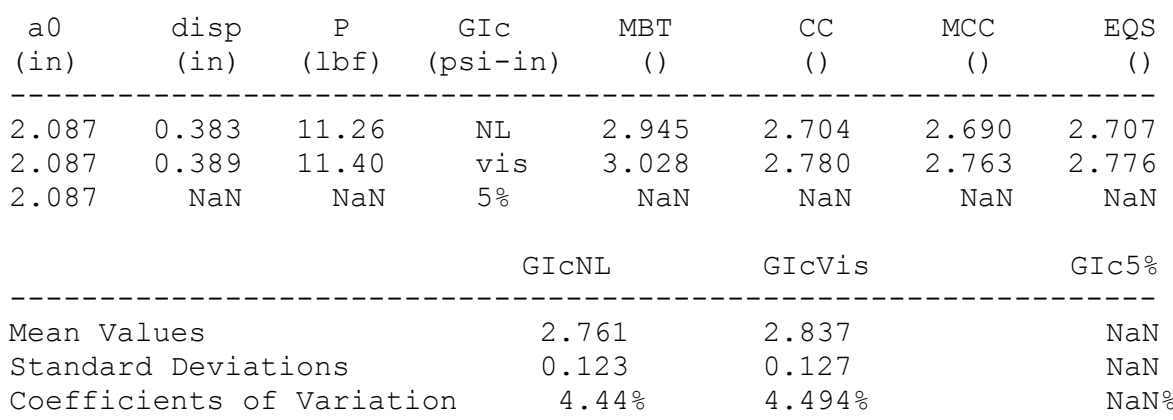

Crack Initiation at Precrack:

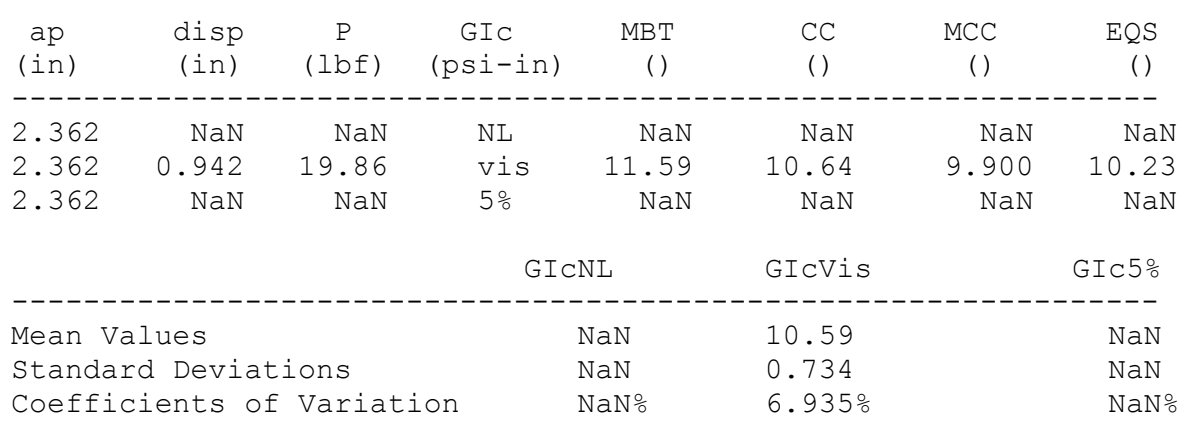

Crack Propagation Values:

\begin{tabular}{|c|c|c|c|c|c|c|c|}
\hline $\begin{array}{l}\mathrm{ap} \\
(\mathrm{in})\end{array}$ & $\begin{array}{l}\text { disp } \\
(\text { in) }\end{array}$ & $\begin{array}{c}P \\
(1 \mathrm{bf})\end{array}$ & $\begin{array}{c}\text { GIC } \\
(p s i-i n)\end{array}$ & $\begin{array}{c}\mathrm{MBT} \\
()\end{array}$ & $\begin{array}{l}\mathrm{CC} \\
()\end{array}$ & $\begin{array}{c}\mathrm{MCC} \\
()\end{array}$ & $\begin{array}{r}E Q S \\
(\text { ) }\end{array}$ \\
\hline & & & & & & & $?$ \\
\hline 2.106 & 0.509 & 13.58 & Prop & 4.624 & 4.246 & $4 \cdot 126$ & $\begin{array}{l}3.962 \\
7\end{array}$ \\
\hline 2.146 & 0.743 & 18.18 & Prop & 8.623 & 7.917 & 7.616 & 7.169 \\
\hline 2.165 & 0.787 & 18.64 & Prop & 9.231 & 8.475 & 8.140 & 7.637 \\
\hline 2.264 & 0.831 & 19.03 & Prop & 9.518 & 8.739 & 8.677 & 8.702 \\
\hline 2.402 & 1.020 & 21.21 & Prop & 12.06 & 11.07 & 11.30 & 11.95 \\
\hline 2.913 & 1.358 & 15.22 & Prop & 9.415 & 8.644 & 8.704 & 8.976 \\
\hline 2.953 & 1.406 & 15.66 & Prop & 9.862 & 9.055 & 9.222 & 9.728 \\
\hline 2.992 & 1.426 & 15.80 & Prop & 9.962 & 9.147 & 9.424 & 10.18 \\
\hline 3.327 & 1.810 & 14.24 & Prop & 10.01 & 9.191 & 9.393 & 9.976 \\
\hline 3.366 & 1.876 & 14.68 & Prop & 10.51 & 9.651 & 9.962 & 10.8 \\
\hline 3.917 & 2.245 & 10.99 & Prop & 8.073 & 7.412 & 7.613 & 8.167 \\
\hline 3.937 & 2.279 & 10.99 & Prop & 8.136 & 7.470 & 7.673 & 8.23 \\
\hline 4.095 & 2.876 & 12.34 & Prop & 10.42 & 9.570 & 9.833 & 10.56 \\
\hline
\end{tabular}

\begin{tabular}{|c|c|c|c|c|}
\hline & GIC-MBT & $\mathrm{GIC}-\mathrm{CC}$ & GIC-MCC & GIC-EQS \\
\hline & ---7 , & 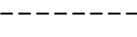 & & ------ \\
\hline Mean Val & 9.266 & 8.507 & 8.591 & 8.925 \\
\hline Standard Deviations & 1.750 & 1.610 & 1.720 & 2.020 \\
\hline Coefficients of Variation & $18.91 \%$ & $18.91 \%$ & $19.99 \%$ & $22.66 \%$ \\
\hline
\end{tabular}


T E S T $\mathrm{N}$ U $\mathrm{M}$ B E $\mathrm{R}$ :

10-3

Crack Initiation at Insert:

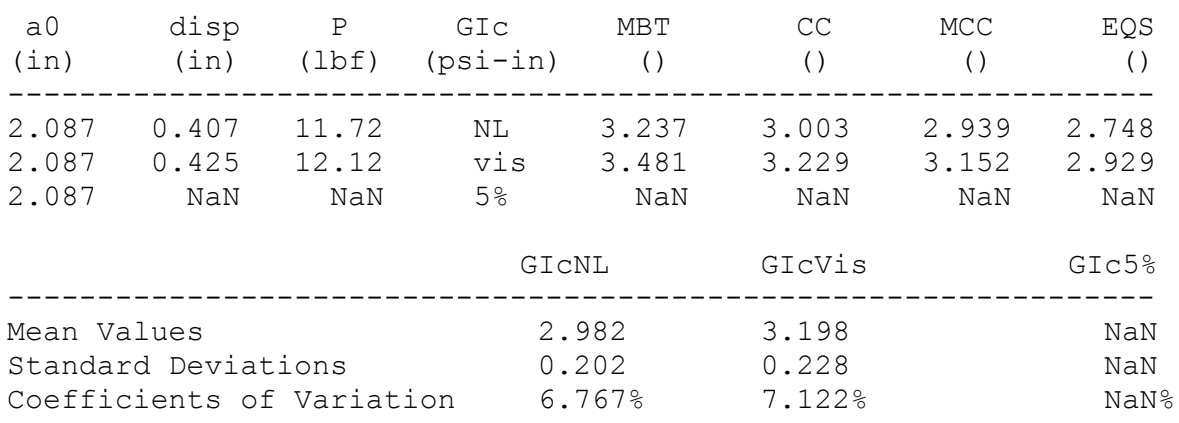

Crack Initiation at Precrack:

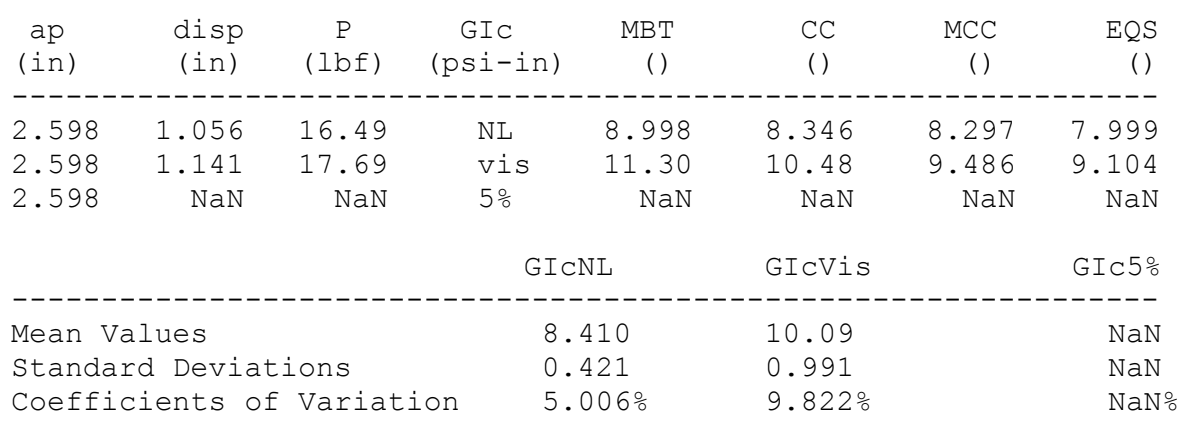

Crack Propagation Values:

\begin{tabular}{|c|c|c|c|c|c|c|c|}
\hline $\begin{array}{l}\mathrm{ap} \\
(\mathrm{in})\end{array}$ & $\begin{array}{l}\text { disp } \\
(i n)\end{array}$ & $\begin{array}{c}P \\
(1 \mathrm{bf})\end{array}$ & $\begin{array}{c}\text { GIC } \\
(p s i-i n)\end{array}$ & $\begin{array}{c}\mathrm{MBT} \\
()\end{array}$ & $\begin{array}{l}\mathrm{CC} \\
()\end{array}$ & $\begin{array}{c}\mathrm{MCC} \\
()\end{array}$ & $\begin{array}{r}E Q S \\
(\text { ) }\end{array}$ \\
\hline & & م & ------- & & & ( & \\
\hline 2.146 & 0.685 & 17.40 & Prop & 7.624 & 7.071 & 6.826 & 6.207 \\
\hline 2.185 & 0.755 & 18.10 & Prop & 8.523 & 7.905 & 7.623 & 6.915 \\
\hline 2.205 & 0.794 & 18.76 & Prop & 9.166 & 8.502 & 8.234 & 7.536 \\
\hline 2.244 & 0.846 & 19.49 & Prop & 9.922 & 9.203 & 8.996 & 8.385 \\
\hline 2.579 & 0.849 & 16.85 & Prop & 7.626 & 7.074 & 7.562 & 8.429 \\
\hline 3.012 & 1.582 & 15.93 & Prop & 10.80 & 10.01 & 9.970 & 9.640 \\
\hline 3.031 & 1.589 & 15.93 & Prop & 10.78 & 9.995 & 10.00 & 9.770 \\
\hline 3.583 & 1.959 & 12.19 & Prop & 8.569 & 7.948 & 8.017 & 7.958 \\
\hline 3.602 & 2.283 & 13.82 & Prop & 10.82 & 10.04 & 10.09 & 9.941 \\
\hline 3.622 & 2.290 & 13.88 & Prop & 10.85 & 10.06 & 10.17 & 10.13 \\
\hline 4.606 & 3.188 & 9.958 & Prop & 8.324 & 7.721 & 7.957 & 8.247 \\
\hline 4.646 & 3.283 & 10.28 & Prop & 8.707 & 8.076 & 8.402 & 8.873 \\
\hline
\end{tabular}

\begin{tabular}{|c|c|c|c|c|}
\hline & GIC-MBT & $\mathrm{GIC}-\mathrm{CC}$ & $\mathrm{GIC}-\mathrm{MCC}$ & GIC-EQS \\
\hline Mean Values & 9.308 & 8.634 & 8.654 & 8.503 \\
\hline Standard Deviations & 1.260 & 1.170 & 1.160 & 1.240 \\
\hline Coefficients of Variation & $13.58 \%$ & $13.58 \%$ & $13.38 \%$ & $14.57 \%$ \\
\hline
\end{tabular}


T E S T N U M B E R :

$10-4$

Crack Initiation at Insert:

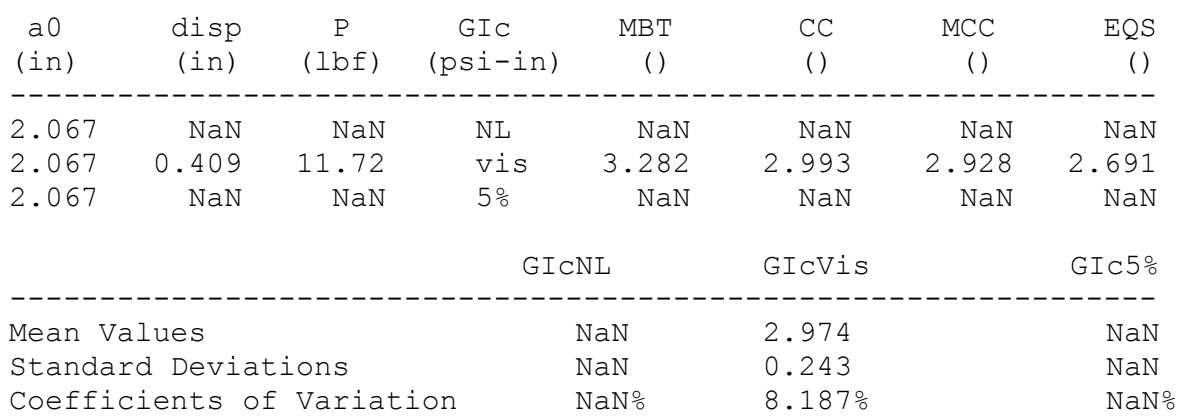

Crack Initiation at Precrack:

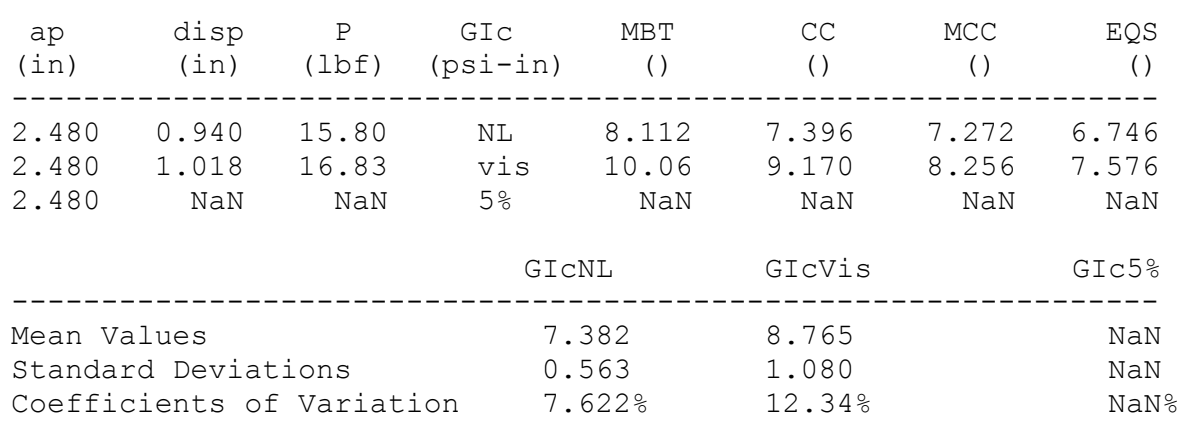

Crack Propagation Values:

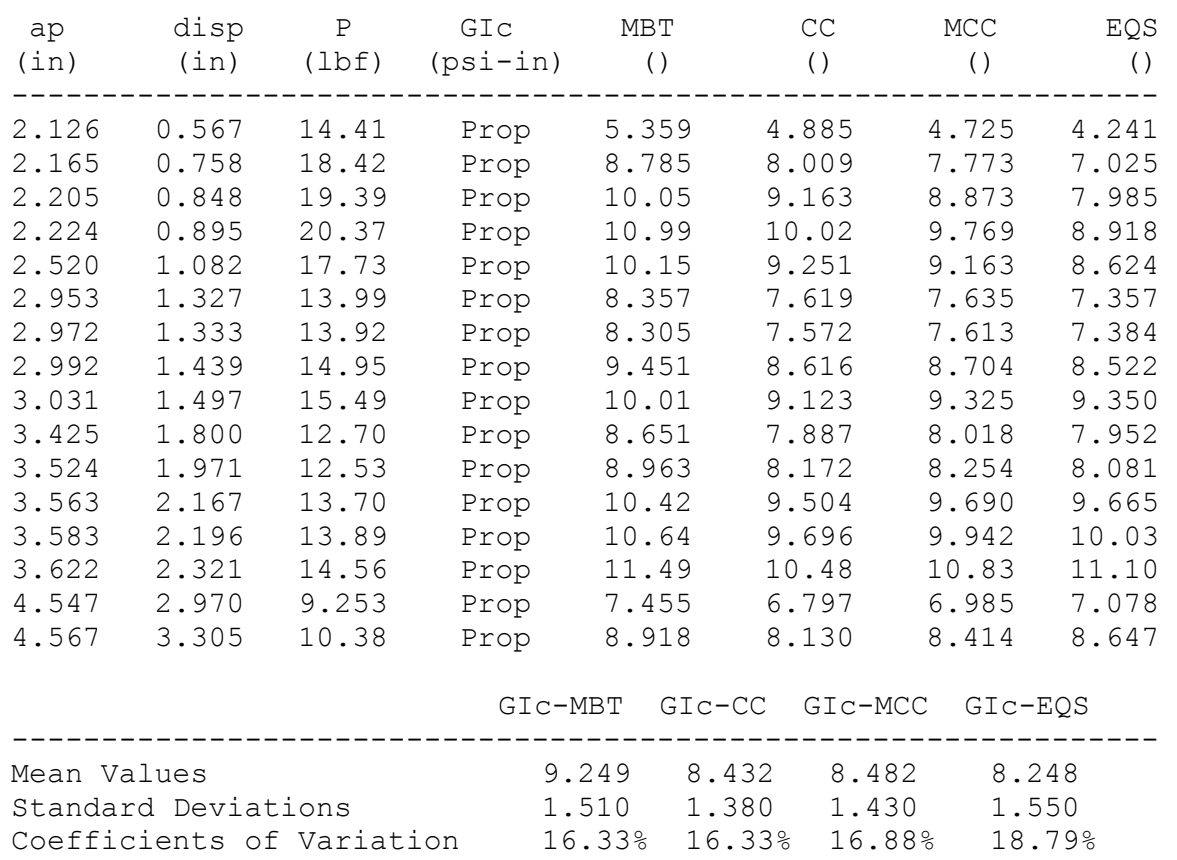


T E S T $N$ U M B E R :

$10-5$

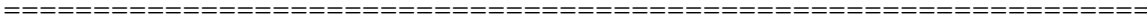

Crack Initiation at Insert:

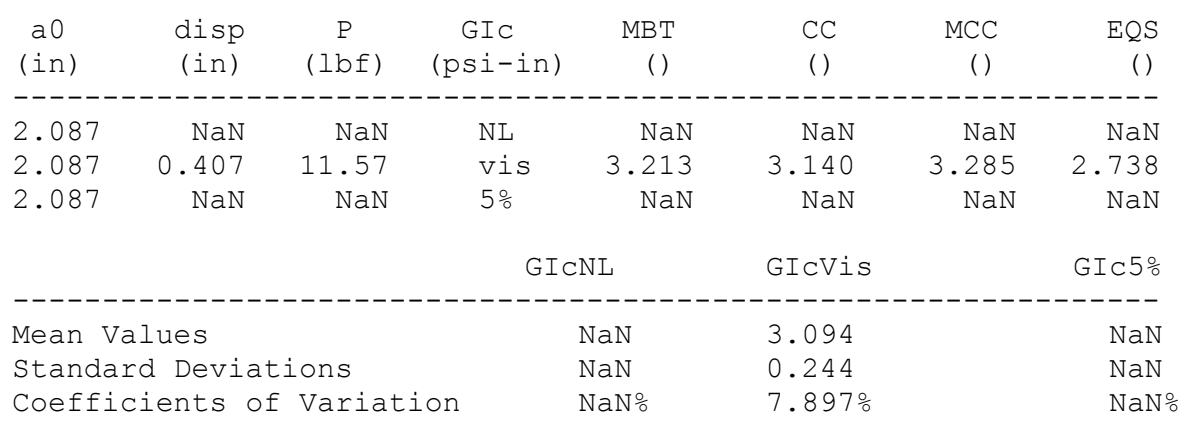

Crack Initiation at Precrack:

\begin{tabular}{|c|c|c|c|c|c|c|c|}
\hline $\begin{array}{l}\mathrm{ap} \\
(\mathrm{in})\end{array}$ & $\begin{array}{l}\text { disp } \\
(i n)\end{array}$ & $\begin{array}{c}\mathrm{P} \\
(1 \mathrm{~b} f)\end{array}$ & $\begin{array}{c}\text { GIC } \\
(p s i-i n)\end{array}$ & $\begin{array}{c}\mathrm{MBT} \\
()\end{array}$ & $\begin{array}{l}\mathrm{CC} \\
()\end{array}$ & $\begin{array}{c}\mathrm{MCC} \\
()\end{array}$ & $\begin{array}{c}\text { EQS } \\
()\end{array}$ \\
\hline & & ------ & & & ------ & ------ & \\
\hline 3.268 & $\mathrm{NaN}$ & $\mathrm{NaN}$ & $\mathrm{NL}$ & $\mathrm{NaN}$ & $\mathrm{NaN}$ & $\mathrm{NaN}$ & $\mathrm{NaN}$ \\
\hline 3.268 & 1.289 & 9.622 & vis & 5.560 & 5.434 & 5.312 & 4.452 \\
\hline 3.268 & $\mathrm{NaN}$ & $\mathrm{NaN}$ & $5 \%$ & $\mathrm{NaN}$ & $\mathrm{NaN}$ & $\mathrm{NaN}$ & $\mathrm{NaN}$ \\
\hline \multicolumn{7}{|c|}{ GICNL } & GIC 5\% \\
\hline & & & ------- & -------- & ------ & ------ & ----- \\
\hline \multicolumn{7}{|c|}{ Mean Values } & $\mathrm{NaN}$ \\
\hline \multicolumn{4}{|c|}{ Standard Deviations } & $\mathrm{NaN}$ & 0.502 & & $\mathrm{NaN}$ \\
\hline \multicolumn{4}{|c|}{ Coefficients of Variation } & $\mathrm{NaN} \%$ & $9.676 \%$ & & \\
\hline
\end{tabular}

Crack Propagation Values:

\begin{tabular}{|c|c|c|c|c|c|c|c|}
\hline $\begin{array}{c}\text { ap } \\
(i n)\end{array}$ & $\begin{array}{l}\text { disp } \\
(\text { in) }\end{array}$ & $\begin{array}{c}\mathrm{P} \\
(\mathrm{lbf})\end{array}$ & $\begin{array}{c}\text { GIC } \\
(p s i-i n)\end{array}$ & $\begin{array}{c}\text { MBT } \\
()\end{array}$ & $\begin{array}{l}\mathrm{CC} \\
()\end{array}$ & $\begin{array}{c}\mathrm{MCC} \\
()\end{array}$ & $\begin{array}{r}E Q S \\
(\text { ) }\end{array}$ \\
\hline & & & & & & -7 & \\
\hline 2.165 & 0.649 & 16.39 & Prop & 6.825 & 6.670 & 6.959 & 5.775 \\
\hline 2.244 & 0.924 & 21.69 & Prop & 12.00 & 11.73 & 12.37 & 10.49 \\
\hline 2.264 & 0.955 & 22.21 & Prop & 12.54 & 12.26 & 13.00 & 11.16 \\
\hline 2.303 & 1.070 & 24.38 & Prop & 14.94 & 14.60 & 15.65 & 13.71 \\
\hline 3.307 & 1.721 & 12.92 & Prop & 8.777 & 8.578 & 9.120 & 7.858 \\
\hline 3.346 & 1.802 & 13.53 & Prop & 9.445 & 9.231 & 9.933 & 8.766 \\
\hline 3.543 & 2.055 & 12.65 & Prop & 9.358 & 9.145 & 9.752 & 8.452 \\
\hline 3.563 & 2.397 & 14.83 & Prop & 12.15 & 11.87 & 12.75 & 11.21 \\
\hline 3.583 & 3.398 & 10.06 & Prop & 9.561 & 9.344 & 7.894 & 4.293 \\
\hline 4.665 & 3.117 & 9.061 & Prop & 7.459 & 7.289 & 7.969 & 7.260 \\
\hline 4.685 & 3.642 & 10.72 & Prop & 9.641 & 9.423 & 10.39 & 9.624 \\
\hline 4.705 & 3.998 & 11.65 & Prop & 10.90 & 10.65 & 11.76 & 10.91 \\
\hline
\end{tabular}

\begin{tabular}{|c|c|c|c|c|}
\hline & GIC-MBT & $\mathrm{GIC}-\mathrm{CC}$ & GIC-MCC & GIC-EQS \\
\hline & & & & \\
\hline Mean Values & 10.30 & 10.07 & 10.63 & 9.1 \\
\hline Standard Deviations & 2.300 & 2.250 & 2.550 & 2.610 \\
\hline Coefficients of Variation & $22.35 \%$ & $22.35 \%$ & $23.95 \%$ & $28.62 \%$ \\
\hline
\end{tabular}


T E S T N U M B E R :

$10-6$

Crack Initiation at Insert:

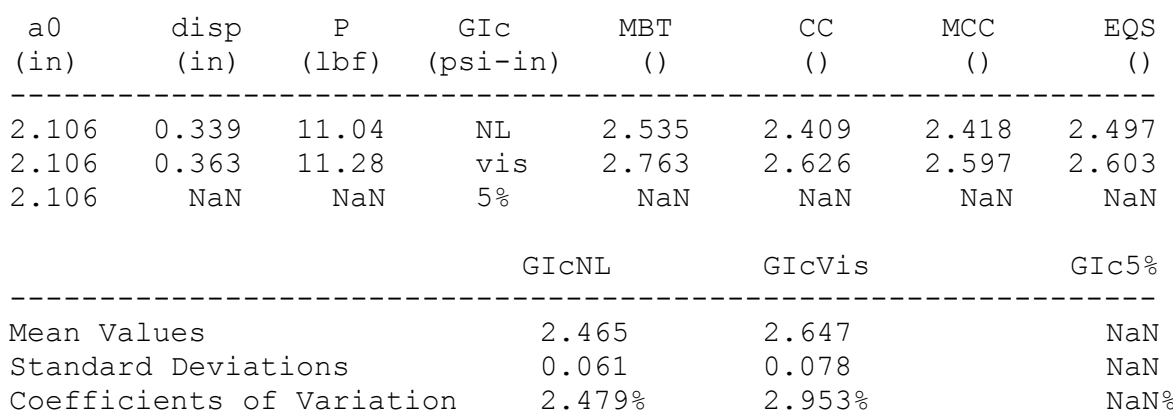

Crack Initiation at Precrack:

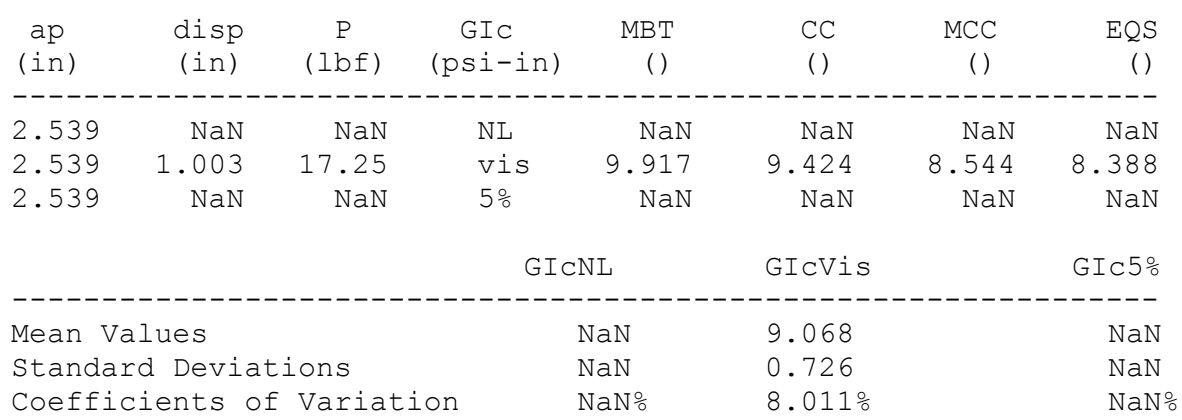

Crack Propagation Values:

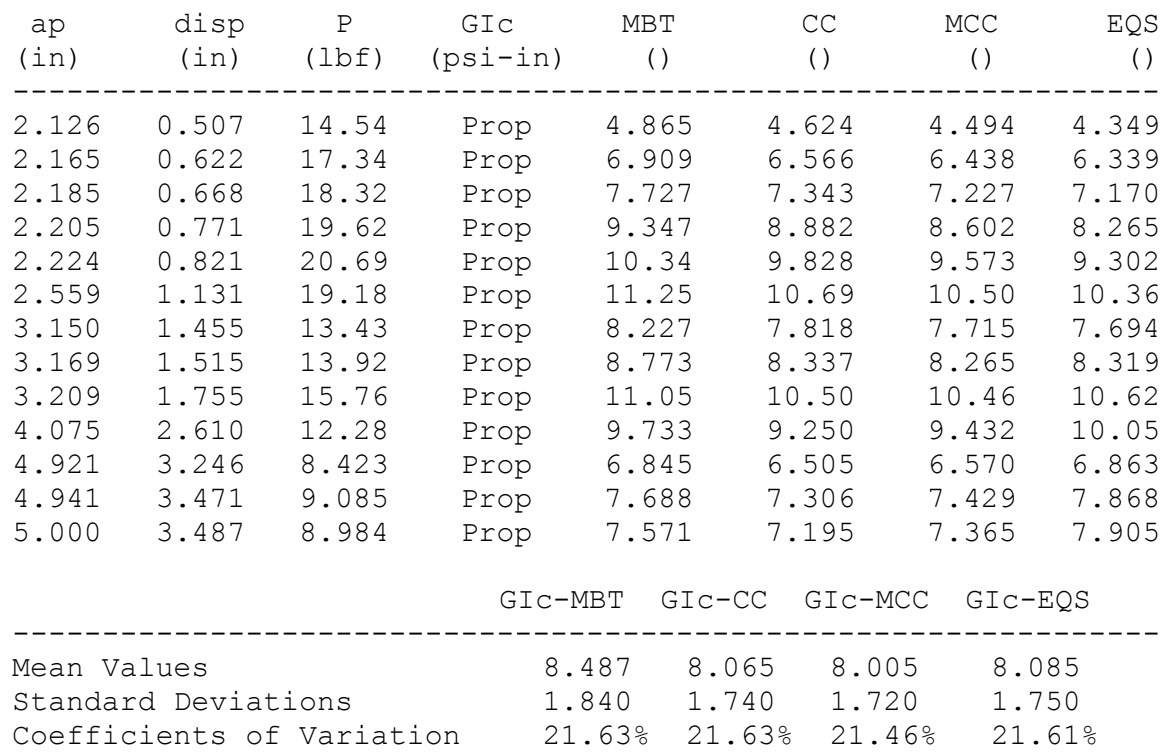


P L A T E S T A T I S T I C S : P L A T E 10

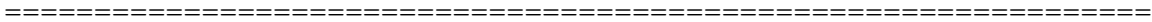

Crack Initiation at Insert

\begin{tabular}{|c|c|c|c|c|c|c|c|}
\hline $\begin{array}{c}\mathrm{a} 0 \\
(\mathrm{in})\end{array}$ & $\begin{array}{l}\text { disp } \\
(\text { in) }\end{array}$ & $\begin{array}{c}\mathrm{P} \\
(1 \mathrm{bf})\end{array}$ & $\begin{array}{c}\text { GIC } \\
(\mathrm{psi}-i n)\end{array}$ & $\begin{array}{c}\mathrm{MBT} \\
()\end{array}$ & $\begin{array}{l}\mathrm{CC} \\
()\end{array}$ & $\begin{array}{c}\mathrm{MCC} \\
()\end{array}$ & $\begin{array}{c}\text { EQS } \\
(\text { ) }\end{array}$ \\
\hline \multicolumn{8}{|c|}{ Mean Values----------------------------------------------------- } \\
\hline & 0.377 & 11.34 & $\mathrm{NL}$ & 2.906 & 2.705 & 2.683 & 2.651 \\
\hline 90 & 0.378 & 11.04 & vis & 2.877 & 2.697 & 2.688 & 2.5 \\
\hline 090 & NaN & $\mathrm{NaN}$ & $5 \%$ & $\mathrm{NaN}$ & NaN & $\mathrm{NaN}$ & $\mathrm{NaN}$ \\
\hline \multicolumn{8}{|c|}{ Standard Deviations--------------------------------------------- } \\
\hline $.48 e-02$ & 0.034 & 0.352 & $\mathrm{NL}$ & 0.353 & 0.297 & 0.261 & 0.135 \\
\hline $8 e-02$ & 0.055 & 1.450 & vis & 0.721 & 0.668 & 0.677 & 0.56 \\
\hline $48 e-02$ & 0.034 & $\mathrm{NaN}$ & $5 \%$ & $\mathrm{NaN}$ & $\mathrm{NaN}$ & $\mathrm{NaN}$ & $\mathrm{NaN}$ \\
\hline \multicolumn{8}{|c|}{ Coefficients of Variation (응) ------------------------------------ } \\
\hline $10 e-01$ & 9.131 & 3.104 & NL & 12.15 & 10.98 & 9.715 & \\
\hline $0 e-01$ & 14.52 & 13.18 & vis & 25.05 & 24.76 & 25.18 & 22. \\
\hline $7.10 e-01$ & NaN & $\mathrm{NaN}$ & $5 \%$ & $\mathrm{NaN}$ & $\mathrm{NaN}$ & $\mathrm{NaN}$ & \\
\hline
\end{tabular}

Crack Initiation at Precrack

\begin{tabular}{|c|c|c|c|c|c|c|c|}
\hline $\begin{array}{l}\operatorname{ap} \\
(\mathrm{in})\end{array}$ & $\begin{array}{l}\text { disp } \\
(\text { in) }\end{array}$ & $\begin{array}{c}\mathrm{P} \\
(1 \mathrm{bf})\end{array}$ & $\begin{array}{c}\text { GIC } \\
(p s i-i n)\end{array}$ & $\begin{array}{c}\text { MBT } \\
()\end{array}$ & $\begin{array}{l}\mathrm{CC} \\
()\end{array}$ & $\begin{array}{c}\mathrm{MCC} \\
()\end{array}$ & $\begin{array}{c}\text { EQS } \\
()\end{array}$ \\
\hline \multicolumn{8}{|c|}{ Mean Values------------------------------------------------------ } \\
\hline 2.720 & 1.099 & 15.22 & $\mathrm{NL}$ & 8.198 & 7.609 & 7.596 & 7.414 \\
\hline 2.720 & 1.139 & 15.92 & vis & 9.669 & 9.038 & 8.297 & 8.021 \\
\hline 2.720 & NaN & $\mathrm{NaN}$ & $5 \%$ & $\mathrm{NaN}$ & $\mathrm{NaN}$ & $\mathrm{NaN}$ & NaN \\
\hline Standard & \multicolumn{7}{|c|}{ Deviations ---------------------------------------------} \\
\hline $3.62 e-01$ & 0.185 & 1.632 & $\mathrm{NL}$ & 0.762 & 0.658 & 0.608 & \\
\hline $3.62 e-01$ & 0.193 & 3.568 & vis & 2.166 & 1.887 & 1.610 & 1.9 \\
\hline $3.62 e-01$ & 0.185 & $\mathrm{NaN}$ & $5 \%$ & $\mathrm{NaN}$ & $\mathrm{NaN}$ & $\mathrm{NaN}$ & $\mathrm{NaN}$ \\
\hline \multicolumn{8}{|c|}{ Coefficients of Variation $\left(\frac{\circ}{\circ}\right)------------------------------------1$} \\
\hline 1. $33 e+01$ & 16.78 & 10.72 & $\mathrm{NL}$ & 9.291 & 8.644 & 8.003 & 8.505 \\
\hline 1. $33 e+01$ & 16.97 & 22.42 & vis & 22.40 & 20.88 & 19.40 & 24.45 \\
\hline 1.33e+01 & $\mathrm{NaN}$ & $\mathrm{NaN}$ & $5 \%$ & $\mathrm{NaN}$ & $\mathrm{NaN}$ & $\mathrm{NaN}$ & NaN \\
\hline
\end{tabular}

Crack Propagation Values

\begin{tabular}{|c|c|c|c|c|}
\hline & GIC-MBT & $\mathrm{IC}-\mathrm{CC}$ & GIC-MCC & GIC-EQS \\
\hline Mean Values & 9.246 & 8.683 & 8.796 & 8.558 \\
\hline Standard Deviations & 1.780 & 1.680 & 1.740 & 1.840 \\
\hline Coefficients of Variation & $19.26 \%$ & $19.26 \%$ & $19.68 \%$ & $21.42 \%$ \\
\hline \multirow[t]{2}{*}{ Slopes and Intercepts } & \multirow{2}{*}{\multicolumn{2}{|c|}{ Delta }} & & \multirow[b]{2}{*}{ A1 } \\
\hline & & & $\mathrm{n}$ & \\
\hline Mean Values & \multirow{3}{*}{\multicolumn{2}{|c|}{$\begin{array}{c}-2.414 e-017 \\
5.91 e-017 \\
-244.9 \%\end{array}$}} & 2.816 & 51.98 \\
\hline Standard Deviations & & & 0.073 & 2.560 \\
\hline Coefficients of Variation & & & $2.595 \%$ & $4.922 \%$ \\
\hline
\end{tabular}




\section{H.6 Plate}

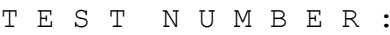

11-1

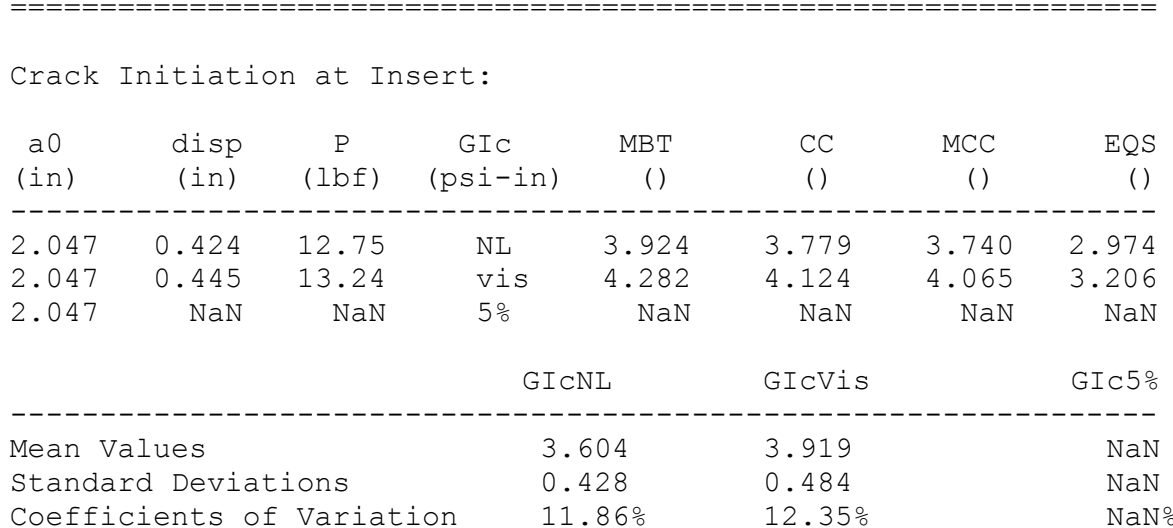

Crack Initiation at Precrack:

\begin{tabular}{|c|c|c|c|c|c|c|c|}
\hline $\begin{array}{l}\mathrm{ap} \\
(\mathrm{in})\end{array}$ & $\begin{array}{l}\text { disp } \\
\text { (in) }\end{array}$ & $\begin{array}{c}\mathrm{P} \\
(1 \mathrm{~b} f)\end{array}$ & $\begin{array}{c}\text { GIC } \\
(p s i-i n)\end{array}$ & $\begin{array}{c}\mathrm{MBT} \\
()\end{array}$ & $\begin{array}{l}\mathrm{CC} \\
()\end{array}$ & $\begin{array}{c}\mathrm{MCC} \\
()\end{array}$ & EQS \\
\hline 071 & 0.917 & 8.270 & $\mathrm{NL}$ & 3.674 & 3.539 & 3.515 & \\
\hline .071 & 1.007 & 8.856 & vis & 4.323 & 4.164 & 3.891 & \\
\hline 3.071 & $\mathrm{NaN}$ & $\mathrm{NaN}$ & $5 \%$ & $\mathrm{NaN}$ & $\mathrm{NaN}$ & $\mathrm{NaN}$ & \\
\hline
\end{tabular}

\begin{tabular}{|c|c|c|c|}
\hline & GICNL & GICVis & GIC $5 \%$ \\
\hline Mean Values & 3.386 & 3.861 & $\mathrm{NaN}$ \\
\hline Standard Deviations & 0.386 & 0.560 & $\mathrm{NaN}$ \\
\hline Coefficients of Variation & $11.4 \%$ & $14.5 \%$ & $\mathrm{NaN} \circ$ \\
\hline
\end{tabular}

Crack Propagation Values:

\begin{tabular}{|c|c|c|c|c|c|c|c|}
\hline $\begin{array}{l}\mathrm{ap} \\
(\mathrm{in})\end{array}$ & $\begin{array}{l}\text { disp } \\
(i n)\end{array}$ & $\begin{array}{c}P \\
(I b f)\end{array}$ & $\begin{array}{c}\text { GIC } \\
(p s i-i n)\end{array}$ & $\begin{array}{c}\mathrm{MBT} \\
(\mathbf{)}\end{array}$ & $\begin{array}{l}\mathrm{CC} \\
()\end{array}$ & $\begin{array}{c}\mathrm{MCC} \\
()\end{array}$ & $\begin{array}{c}E Q S \\
(\text { ) }\end{array}$ \\
\hline 2.500 & 0.696 & 11.21 & Prop & 4.641 & 4.470 & 4. 386 & \\
\hline 3.661 & 1.402 & 7.663 & Prop & 4.365 & 4.204 & 4.214 & 3.438 \\
\hline 4.409 & 1.952 & 6.265 & Prop & 4.128 & 3.975 & 4.018 & 3.333 \\
\hline
\end{tabular}

\begin{tabular}{|c|c|c|c|c|}
\hline & GIC-MBT & $\mathrm{GIC}-\mathrm{CC}$ & GIC-MCC & GIC-EQS \\
\hline lean Values & 4.378 & 4.216 & 4.206 & 3.400 \\
\hline Standard Deviations & 0.257 & 0.248 & 0.184 & 0.058 \\
\hline Coefficients of Variation & $5.872 \%$ & $5.872 \%$ & $4.372 \%$ & $1.700 \%$ \\
\hline
\end{tabular}




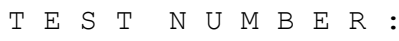

$11-2$

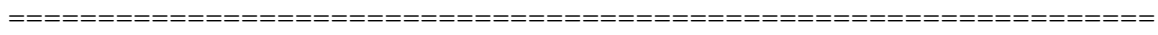

Crack Initiation at Insert:

\begin{tabular}{|c|c|c|c|c|c|c|c|}
\hline $\begin{array}{c}\mathrm{a} 0 \\
(\mathrm{in})\end{array}$ & $\begin{array}{l}\text { disp } \\
(i n)\end{array}$ & $\begin{array}{c}\mathrm{P} \\
(1 \mathrm{bf})\end{array}$ & $\begin{array}{c}\text { GIC } \\
(p s i-i n)\end{array}$ & $\begin{array}{r}\text { MBT } \\
()\end{array}$ & $\begin{array}{l}\text { CC } \\
()\end{array}$ & $\begin{array}{c}\mathrm{MCC} \\
()\end{array}$ & $\begin{array}{c}\text { EQS } \\
()\end{array}$ \\
\hline \multicolumn{8}{|c|}{ 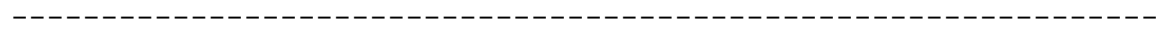 } \\
\hline 2.047 & 0.406 & 13.25 & $\mathrm{NL}$ & 3.793 & 3.727 & 3.728 & 2.963 \\
\hline 2.047 & 0.427 & 13.80 & vis & 4.151 & 4.079 & 4.066 & 3.211 \\
\hline \multirow[t]{2}{*}{2.047} & $\mathrm{NaN}$ & $\mathrm{NaN}$ & $5 \%$ & $\mathrm{NaN}$ & $\mathrm{NaN}$ & $\mathrm{NaN}$ & $\mathrm{NaN}$ \\
\hline & & & \multicolumn{2}{|c|}{ GICNL } & GIcVis & & GIC $5 \%$ \\
\hline \multicolumn{8}{|c|}{----------------------------------------------------------------} \\
\hline \multicolumn{5}{|c|}{ Mean Values $\quad 3.552$} & \multicolumn{2}{|c|}{3.877} & \\
\hline \multicolumn{3}{|c|}{ Standard Deviations } & & 94 & \multicolumn{2}{|l|}{0.446} & \\
\hline \multicolumn{3}{|c|}{ Coefficients of Vari } & & $10 \%$ & \multicolumn{2}{|l|}{$11.50 \%$} & \\
\hline
\end{tabular}

Crack Initiation at Precrack:

\begin{tabular}{|c|c|c|c|c|c|c|c|}
\hline $\begin{array}{l}\text { ap } \\
(i n)\end{array}$ & $\begin{array}{l}\text { disp } \\
(i n)\end{array}$ & $\begin{array}{c}P \\
(1 \mathrm{bf})\end{array}$ & $\begin{array}{c}\text { GIC } \\
(p s i-i n)\end{array}$ & $\begin{array}{c}\mathrm{MBT} \\
(\text { ) }\end{array}$ & $\begin{array}{l}\mathrm{CC} \\
()\end{array}$ & $\begin{array}{c}\mathrm{MCC} \\
()\end{array}$ & \\
\hline 2.421 & 0.555 & 10.67 & $\mathrm{NL}$ & 3.528 & 3.466 & 3.437 & 2.6 \\
\hline 2.421 & 0.603 & 11.38 & vis & 4.088 & 4.016 & 3.813 & 2. \\
\hline 2.421 & $\mathrm{NaN}$ & $\mathrm{NaN}$ & $5 \%$ & $\mathrm{NaN}$ & $\mathrm{NaN}$ & $\mathrm{NaN}$ & \\
\hline
\end{tabular}

\begin{tabular}{|c|c|c|c|}
\hline & GICNL & GICVis & GIC $5 \%$ \\
\hline Mean Values & 3.279 & 3.715 & $\mathrm{NaN}$ \\
\hline Standard Deviations & 0.398 & 0.528 & $\mathrm{NaN}$ \\
\hline Coefficients of Variation & $12.15 \%$ & $14.21 \%$ & $\mathrm{NaN} \%$ \\
\hline
\end{tabular}

Crack Propagation Values:

\begin{tabular}{|c|c|c|c|c|c|c|c|}
\hline $\begin{array}{c}\mathrm{ap} \\
(\mathrm{in})\end{array}$ & $\begin{array}{l}\text { disp } \\
(i n)\end{array}$ & $\begin{array}{c}P \\
(1 \mathrm{bf})\end{array}$ & $\begin{array}{c}\text { GIC } \\
(p s i-i n)\end{array}$ & $\begin{array}{c}\mathrm{MBT} \\
(\mathbf{)}\end{array}$ & $\begin{array}{l}\mathrm{CC} \\
()\end{array}$ & $\begin{array}{c}\mathrm{MCC} \\
()\end{array}$ & $\begin{array}{r}E Q S \\
(\text { ) }\end{array}$ \\
\hline---- & --- & & -------- & ----- & ------ & ----- & ---- \\
\hline 2.874 & 0.858 & 10.06 & Prop & 4.328 & 4.253 & 4.245 & 3.361 \\
\hline 3.504 & 1.246 & 8.170 & Prop & 4.190 & 4.117 & 4.128 & 3.298 \\
\hline
\end{tabular}

\begin{tabular}{|c|c|c|c|c|}
\hline & GIC-MBT & $\mathrm{GIC}-\mathrm{CC}$ & $\mathrm{GIC}-\mathrm{MCC}$ & GIC-EQS \\
\hline & & & & ค \\
\hline Mean Values & 4.259 & 4.185 & 4.187 & 3.329 \\
\hline Standard Deviations & 0.098 & 0.0962 & 0.083 & 0.045 \\
\hline Coefficients of Variation & $2.299 \%$ & $2.299 \%$ & $1.982 \%$ & $1.347 \%$ \\
\hline
\end{tabular}




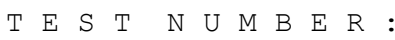

$11-3$

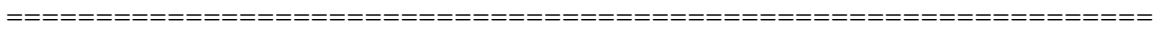

Crack Initiation at Insert:

\begin{tabular}{|c|c|c|c|c|c|c|c|}
\hline $\begin{array}{c}\mathrm{a} 0 \\
(\mathrm{in})\end{array}$ & $\begin{array}{l}\text { disp } \\
(i n)\end{array}$ & $\begin{array}{c}\mathrm{P} \\
(1 \mathrm{bf})\end{array}$ & $\begin{array}{c}\text { GIC } \\
(p s i-i n)\end{array}$ & $\begin{array}{r}\text { MBT } \\
()\end{array}$ & $\begin{array}{l}\text { CC } \\
()\end{array}$ & $\begin{array}{c}\mathrm{MCC} \\
()\end{array}$ & $\begin{array}{c}\text { EQS } \\
()\end{array}$ \\
\hline \multicolumn{8}{|c|}{ 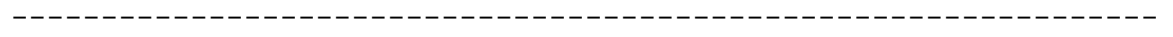 } \\
\hline 2.047 & 0.397 & 12.30 & $\mathrm{NL}$ & 3.454 & 3.202 & 3.136 & 2.573 \\
\hline 2.047 & 0.436 & 13.31 & vis & 4.098 & 3.800 & 3.703 & 3.0 \\
\hline \multirow[t]{2}{*}{2.047} & $\mathrm{NaN}$ & $\mathrm{NaN}$ & $5 \%$ & $\mathrm{NaN}$ & $\mathrm{NaN}$ & $\mathrm{NaN}$ & $\mathrm{NaN}$ \\
\hline & & & \multicolumn{2}{|c|}{ GICNL } & GIcVis & & GIC $5 \%$ \\
\hline \multicolumn{8}{|c|}{----------------------------------------------------------------} \\
\hline \multicolumn{5}{|c|}{ Mean Values $\quad 3.091$} & \multicolumn{2}{|c|}{3.652} & \\
\hline \multicolumn{3}{|c|}{ Standard Deviations } & \multicolumn{2}{|c|}{0.372} & \multicolumn{2}{|l|}{0.461} & \\
\hline \multicolumn{3}{|c|}{ Coefficients of Vari } & & $02 \%$ & \multicolumn{2}{|l|}{$12.62 \%$} & \\
\hline
\end{tabular}

Crack Initiation at Precrack:

\begin{tabular}{|c|c|c|c|c|c|c|c|}
\hline $\begin{array}{c}\text { ap } \\
(i n)\end{array}$ & $\begin{array}{l}\text { disp } \\
(\text { in) }\end{array}$ & $\begin{array}{c}\mathrm{P} \\
(1 \mathrm{bf})\end{array}$ & $\begin{array}{c}\text { GIC } \\
(p s i-i n)\end{array}$ & $\begin{array}{c}\mathrm{MBT} \\
(\mathbf{)}\end{array}$ & $\begin{array}{l}\mathrm{CC} \\
()\end{array}$ & $\begin{array}{c}\mathrm{MCC} \\
()\end{array}$ & \\
\hline 2.539 & 0.618 & 10.10 & $\mathrm{NL}$ & 3.559 & 3.300 & 3.239 & 2.6 \\
\hline 2.539 & 0.646 & 10.50 & vis & 3.865 & 3.583 & 3.381 & 2.7 \\
\hline 2.539 & $\mathrm{NaN}$ & $\mathrm{NaN}$ & $5 \%$ & $\mathrm{NaN}$ & $\mathrm{NaN}$ & NaN & \\
\hline
\end{tabular}

\begin{tabular}{|c|c|c|c|}
\hline & GICNL & GICVis & GIC $5 \%$ \\
\hline Mean Values & 3192 & 3401 & NTaN \\
\hline Standard Deviations & 0.375 & 0.461 & $\mathrm{NaN}$ \\
\hline Coefficients of Variation & $11.74 \%$ & $13.56 \%$ & \\
\hline
\end{tabular}

Crack Propagation Values:

\begin{tabular}{|c|c|c|c|c|c|c|c|}
\hline $\begin{array}{l}\mathrm{ap} \\
(\mathrm{in})\end{array}$ & $\begin{array}{l}\text { disp } \\
(\text { in) }\end{array}$ & $\begin{array}{c}P \\
(1 \mathrm{bf})\end{array}$ & $\begin{array}{c}\text { GIC } \\
(\mathrm{psi-in)}\end{array}$ & $\begin{array}{c}\mathrm{MBT} \\
()\end{array}$ & $\begin{array}{l}\mathrm{CC} \\
()\end{array}$ & $\begin{array}{c}\mathrm{MCC} \\
()\end{array}$ & $\begin{array}{r}E Q S \\
()\end{array}$ \\
\hline 2.894 & 0.893 & 9.250 & Prop & 4.134 & 3.833 & 3.681 & 2.905 \\
\hline 3.346 & 1.176 & 8.493 & Prop & 4.320 & 4.005 & 3.946 & 3.276 \\
\hline 3.858 & 1.457 & 7.370 & Prop & 4.031 & 3.737 & 3.769 & 3.279 \\
\hline 3.976 & 1.479 & 6.955 & Prop & 3.745 & 3.472 & 3.522 & 3.102 \\
\hline 4.016 & 1.642 & 7.465 & Prop & 4.418 & 4.096 & 4.150 & 3.644 \\
\hline 4.232 & 2.095 & 8.371 & Prop & 6.000 & 5.563 & 5.689 & 5.090 \\
\hline
\end{tabular}

\begin{tabular}{|c|c|c|c|c|}
\hline & GIC-MBT & $\mathrm{GIC}-\mathrm{CC}$ & GIC-MCC & GIC-EQS \\
\hline Mean Values & 4.441 & 4.118 & 4.126 & 3.549 \\
\hline Standard Deviations & 0.799 & 0.741 & 0.796 & 0.793 \\
\hline Coefficients of Variation & $17.99 \%$ & $17.99 \%$ & $19.28 \%$ & $22.35 \%$ \\
\hline
\end{tabular}


T E $\quad S \quad T \quad N \quad U$ M $B$ E R :

$11-4$

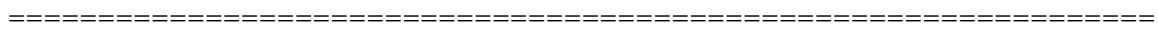

Crack Initiation at Insert:

\begin{tabular}{|c|c|c|c|c|c|c|c|}
\hline $\begin{array}{c}\mathrm{a} 0 \\
(\mathrm{in})\end{array}$ & $\begin{array}{l}\text { disp } \\
(i n)\end{array}$ & $\begin{array}{c}\mathrm{P} \\
(1 \mathrm{bf})\end{array}$ & $\begin{array}{c}\text { GIC } \\
(p s i-i n)\end{array}$ & $\begin{array}{r}\text { MBT } \\
()\end{array}$ & $\begin{array}{l}\text { CC } \\
()\end{array}$ & $\begin{array}{c}\mathrm{MCC} \\
()\end{array}$ & $\begin{array}{c}\text { EQS } \\
()\end{array}$ \\
\hline \multicolumn{8}{|c|}{ 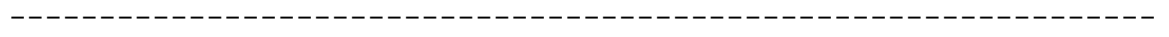 } \\
\hline 2.047 & 0.403 & 12.20 & $\mathrm{NL}$ & 3.561 & 3.370 & 3.327 & 2.758 \\
\hline 2.047 & 0.432 & 12.97 & vis & 4.056 & 3.839 & 3.778 & 3.113 \\
\hline 2.047 & $\mathrm{NaN}$ & $\mathrm{NaN}$ & $5 \%$ & $\mathrm{NaN}$ & $\mathrm{NaN}$ & $\mathrm{NaN}$ & $\mathrm{NaN}$ \\
\hline & & & GIC & & & & GIC $5 \%$ \\
\hline & & & 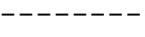 & & & & \\
\hline \multicolumn{5}{|c|}{ Mean Values } & \multicolumn{2}{|l|}{3.696} & \\
\hline \multicolumn{3}{|c|}{ Standard Deviations } & & 46 & \multicolumn{2}{|l|}{0.407} & $\mathrm{NaN}$ \\
\hline \multicolumn{3}{|c|}{ Coefficients of Vari } & on & $63 \%$ & \multicolumn{2}{|l|}{$11.0 \%$} & \\
\hline
\end{tabular}

Crack Initiation at Precrack:

\begin{tabular}{|c|c|c|c|c|c|c|c|}
\hline $\begin{array}{l}\text { ap } \\
(i n)\end{array}$ & $\begin{array}{l}\text { disp } \\
(i n)\end{array}$ & $\begin{array}{c}P \\
(1 \mathrm{bf})\end{array}$ & $\begin{array}{c}\text { GIC } \\
(p s i-i n)\end{array}$ & $\begin{array}{c}\text { MBT } \\
()\end{array}$ & $\begin{array}{l}\mathrm{CC} \\
()\end{array}$ & $\begin{array}{c}\mathrm{MCC} \\
()\end{array}$ & \\
\hline 2.480 & $\mathrm{NaN}$ & $\mathrm{NaN}$ & $\mathrm{NL}$ & $\mathrm{NaN}$ & $\mathrm{NaN}$ & $\mathrm{NaN}$ & \\
\hline 2.480 & 0.6271 & 10.23 & vis & 3.832 & 3.627 & 3.398 & 2. \\
\hline 2.480 & $\mathrm{NaN}$ & $\mathrm{NaN}$ & $5 \%$ & $\mathrm{NaN}$ & NaN & NaN & \\
\hline
\end{tabular}

\begin{tabular}{|c|c|c|c|}
\hline & GICNL & GICVis & GIC $5 \%$ \\
\hline 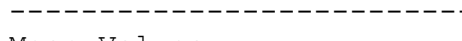 & --- & 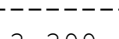 & --- \\
\hline Mean Values & $\mathrm{NaN}$ & 3.399 & $\mathrm{NaN}$ \\
\hline Standard Deviations & $\mathrm{NaN}$ & 0.476 & $\mathrm{NaN}$ \\
\hline Coefficients of Variation & $\mathrm{NaN} \%$ & $13.99 \%$ & $\mathrm{NaN} \circ$ \\
\hline
\end{tabular}

Crack Propagation Values:

\begin{tabular}{|c|c|c|c|c|c|c|c|}
\hline $\begin{array}{l}\mathrm{ap} \\
(\mathrm{in})\end{array}$ & $\begin{array}{l}\text { disp } \\
\text { (in) }\end{array}$ & $\begin{array}{c}P \\
(1 \mathrm{~b} f)\end{array}$ & $\begin{array}{c}\text { GIC } \\
(p s i-i n)\end{array}$ & $\begin{array}{c}\mathrm{MBT} \\
()\end{array}$ & $\begin{array}{l}\mathrm{CC} \\
()\end{array}$ & $\begin{array}{c}\mathrm{MCC} \\
()\end{array}$ & $\begin{array}{c}\text { EQS } \\
()\end{array}$ \\
\hline 2.953 & 0.904 & 9.445 & Prop & 4.284 & 4.055 & 4.049 & 3.437 \\
\hline 3.622 & 1.290 & 7.492 & Prop & 3.954 & 3.742 & 3.769 & 3.253 \\
\hline 4.173 & 1.912 & 7.44 & Prop & 5.052 & 4.782 & 4.855 & 4.259 \\
\hline
\end{tabular}

\begin{tabular}{|c|c|c|c|c|}
\hline & GIC-MBT & $\mathrm{GIC}-\mathrm{CC}$ & GIC-MCC & GIC-EQS \\
\hline Mean Values & 4.430 & 4.193 & 4.224 & 3.650 \\
\hline Standard Deviations & 0.564 & 0.533 & 0.564 & 0.536 \\
\hline Coefficients of Variation & $12.72 \%$ & $12.72 \%$ & $13.35 \%$ & $14.68 \%$ \\
\hline
\end{tabular}




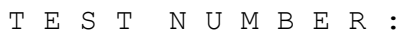

$11-5$

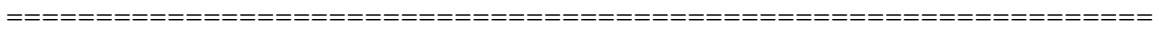

Crack Initiation at Insert:

\begin{tabular}{|c|c|c|c|c|c|c|c|}
\hline $\begin{array}{c}\mathrm{a} 0 \\
(\mathrm{in})\end{array}$ & $\begin{array}{l}\text { disp } \\
(i n)\end{array}$ & $\begin{array}{c}\mathrm{P} \\
(1 \mathrm{bf})\end{array}$ & $\begin{array}{c}\text { GIC } \\
(p s i-i n)\end{array}$ & $\begin{array}{r}\text { MBT } \\
()\end{array}$ & $\begin{array}{l}\text { CC } \\
()\end{array}$ & $\begin{array}{c}\mathrm{MCC} \\
()\end{array}$ & $\begin{array}{c}\text { EQS } \\
()\end{array}$ \\
\hline \multicolumn{8}{|c|}{ 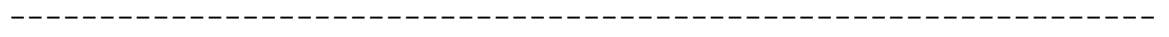 } \\
\hline 2.047 & 0.418 & 12.88 & $\mathrm{NL}$ & 3.905 & 3.914 & 3.984 & 3.040 \\
\hline 2.047 & 0.446 & 13.48 & vis & 4.362 & 4.372 & 4.420 & 3.329 \\
\hline \multirow[t]{2}{*}{2.047} & $\mathrm{NaN}$ & $\mathrm{NaN}$ & $5 \%$ & $\mathrm{NaN}$ & $\mathrm{NaN}$ & $\mathrm{NaN}$ & $\mathrm{NaN}$ \\
\hline & & & \multicolumn{2}{|c|}{ GICNL } & GIcVis & & GIC $5 \%$ \\
\hline \multicolumn{8}{|c|}{----------------------------------------------------------------} \\
\hline \multicolumn{5}{|c|}{ Mean Values $\quad 3.711$} & \multicolumn{2}{|c|}{4.121} & \\
\hline \multicolumn{3}{|c|}{ Standard Deviations } & & 48 & \multicolumn{2}{|l|}{0.528} & \\
\hline \multicolumn{3}{|c|}{ Coefficients of Vari } & & $08 \%$ & \multicolumn{2}{|l|}{$12.82 \%$} & \\
\hline
\end{tabular}

Crack Initiation at Precrack:

\begin{tabular}{|c|c|c|c|c|c|c|c|}
\hline $\begin{array}{c}\text { ap } \\
(i n)\end{array}$ & $\begin{array}{l}\text { disp } \\
(\text { in) }\end{array}$ & $\begin{array}{c}\mathrm{P} \\
(1 \mathrm{bf})\end{array}$ & $\begin{array}{c}\text { GIC } \\
(p s i-i n)\end{array}$ & $\begin{array}{c}\mathrm{MBT} \\
(\mathbf{)}\end{array}$ & $\begin{array}{l}\mathrm{CC} \\
()\end{array}$ & $\begin{array}{c}\mathrm{MCC} \\
()\end{array}$ & \\
\hline 2.441 & 0.585 & 9.613 & $\mathrm{NL}$ & 3.419 & 3.428 & 3.372 & 2. \\
\hline 2.441 & 0.644 & 10.42 & vis & 4.080 & 4.090 & 3.845 & 2. \\
\hline 2.441 & $\mathrm{NaN}$ & $\mathrm{NaN}$ & $5 \%$ & $\mathrm{NaN}$ & NaN & NaN & \\
\hline
\end{tabular}

\begin{tabular}{|c|c|c|c|}
\hline & GICNL & GICVis & GIC $5 \%$ \\
\hline 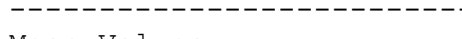 & 3.156 & 3602 & NT \\
\hline Mean Values & 3.156 & 3.683 & $\mathrm{NaN}$ \\
\hline Standard Deviations & 0.501 & 0.654 & $\mathrm{NaN}$ \\
\hline Coefficients of Variation & $15.87 \%$ & $17.76 \%$ & $\mathrm{NaN} \%$ \\
\hline
\end{tabular}

Crack Propagation Values:

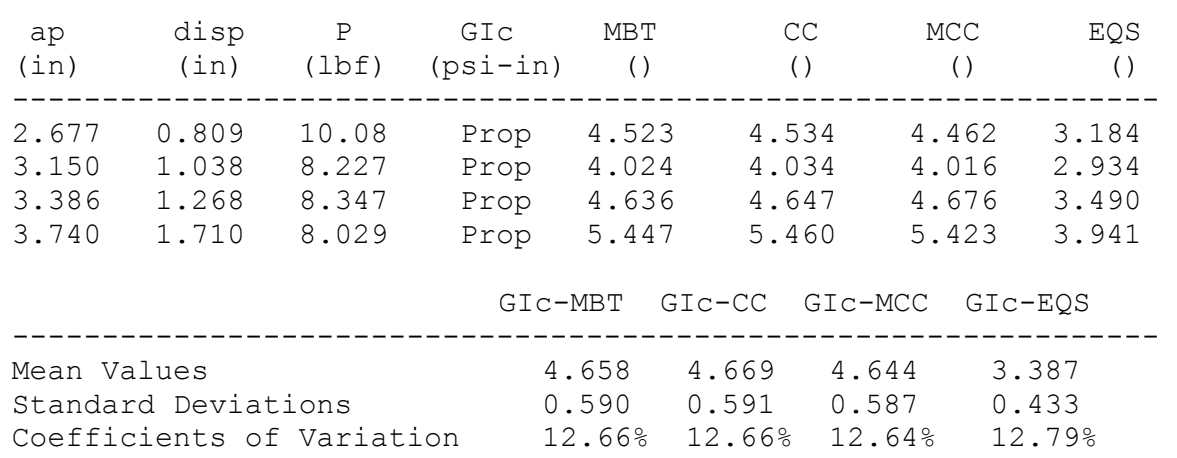


T E S T N U M B E R : 11-6

$==\mathrm{=}=\mathrm{=}=\mathrm{=}=\mathrm{=}=\mathrm{=}=\mathrm{=}=\mathrm{=}=\mathrm{=}=\mathrm{=}=\mathrm{=}=\mathrm{=}=\mathrm{=}=\mathrm{=}=\mathrm{=}=\mathrm{=}=\mathrm{=}=\mathrm{=}=\mathrm{=}=\mathrm{=}=\mathrm{=}=\mathrm{=}=\mathrm{=}=\mathrm{=}=\mathrm{C}$

Crack Initiation at Insert:

\begin{tabular}{|c|c|c|c|c|c|c|c|}
\hline $\begin{array}{l}\mathrm{a} 0 \\
(\mathrm{in})\end{array}$ & $\begin{array}{c}\text { disp } \\
(\text { in) }\end{array}$ & $\begin{array}{c}\mathrm{P} \\
(1 \mathrm{bf})\end{array}$ & $\begin{array}{c}\text { GIC } \\
(\mathrm{psi}-\mathrm{in})\end{array}$ & $\begin{array}{r}\mathrm{MBT} \\
()\end{array}$ & $\begin{array}{l}\mathrm{CC} \\
()\end{array}$ & $\begin{array}{r}\mathrm{MCC} \\
()\end{array}$ & $\begin{array}{c}\text { EQS } \\
()\end{array}$ \\
\hline \multicolumn{8}{|c|}{ 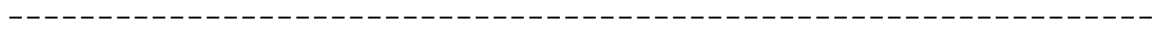 } \\
\hline 2.047 & 0.405 & 12.06 & NL & 3.523 & 3.318 & 3.276 & 2.654 \\
\hline 2.047 & 0.421 & 12.47 & vis & 3.781 & 3.561 & 3.512 & 2.838 \\
\hline 2.047 & $\mathrm{NaN}$ & $\mathrm{NaN}$ & $5 \%$ & $\mathrm{NaN}$ & $\mathrm{NaN}$ & $\mathrm{NaN}$ & $\mathrm{NaN}$ \\
\hline \multicolumn{5}{|c|}{ GICNL } & GICVis & & GIC5\% \\
\hline \multicolumn{5}{|c|}{ Mean Values } & 3.423 & & $\mathbb{N}$ \\
\hline \multicolumn{3}{|c|}{ Standard Deviations } & & 75 & 0.407 & & $\mathrm{NaN}$ \\
\hline \multicolumn{3}{|c|}{ Coefficients of Variation } & on & $76 \%$ & $11.9 \%$ & & $\mathrm{aN} \circ$ \\
\hline
\end{tabular}

Crack Initiation at Precrack:

\begin{tabular}{|c|c|c|c|c|c|c|c|}
\hline $\begin{array}{l}\text { ap } \\
(i n)\end{array}$ & $\begin{array}{l}\text { disp } \\
(\text { in) }\end{array}$ & $\begin{array}{c}P \\
(1 \mathrm{bf})\end{array}$ & $\begin{array}{c}\text { GIC } \\
(p s i-i n)\end{array}$ & $\begin{array}{c}\mathrm{MBT} \\
(\mathrm{)}\end{array}$ & $\begin{array}{l}\mathrm{CC} \\
()\end{array}$ & $\begin{array}{c}\mathrm{MCC} \\
()\end{array}$ & \\
\hline 2.342 & 0.549 & 10.32 & $\mathrm{NL}$ & 3.572 & 3.363 & 3.261 & 2.5 \\
\hline 2.342 & 0.591 & 10.94 & vis & 4.075 & 3.837 & 3.559 & 2.7 \\
\hline 2.342 & $\mathrm{NaN}$ & $\mathrm{NaN}$ & $5 \%$ & $\mathrm{NaN}$ & $\mathrm{NaN}$ & NaN & \\
\hline
\end{tabular}

\begin{tabular}{|c|c|c|c|}
\hline & GICNL & GICVis & GIC $5 \%$ \\
\hline & & & \\
\hline Mean Values & 3.186 & 3.556 & $\mathrm{NaN}$ \\
\hline Standard Deviations & 0.445 & 0.577 & $\mathrm{NaN}$ \\
\hline Coefficients of Variation & $13.97 \%$ & $16.23 \%$ & $\mathrm{NaN} \div$ \\
\hline
\end{tabular}

Crack Propagation Values:

\begin{tabular}{|c|c|c|c|c|c|c|c|}
\hline $\begin{array}{c}\mathrm{ap} \\
(\mathrm{in})\end{array}$ & $\begin{array}{l}\text { disp } \\
(\text { in) }\end{array}$ & $\begin{array}{c}P \\
(1 \mathrm{bf})\end{array}$ & $\begin{array}{c}\text { GIC } \\
(p s i-i n)\end{array}$ & $\begin{array}{c}\mathrm{MBT} \\
(\text { ) }\end{array}$ & $\begin{array}{l}\mathrm{CC} \\
()\end{array}$ & $\begin{array}{c}\mathrm{MCC} \\
()\end{array}$ & $\begin{array}{r}E Q S \\
()\end{array}$ \\
\hline & & & -------- & --- & ------ & ----- & ---- \\
\hline 2.835 & 0.951 & 10.10 & Prop & 5.003 & 4.711 & 4.569 & 3.570 \\
\hline 3.701 & 1.406 & 7.712 & Prop & 4. 326 & 4.074 & 4.138 & 3.547 \\
\hline
\end{tabular}

\begin{tabular}{|c|c|c|c|c|}
\hline & GIC-MBT & $\mathrm{GIC}-\mathrm{CC}$ & $\mathrm{GIC}-\mathrm{MCC}$ & GIC-EQS \\
\hline & & & & \\
\hline Mean Values & 4.665 & 4.392 & 4.353 & 3.559 \\
\hline Standard Deviations & 0.478 & 0.451 & 0.305 & 0.016 \\
\hline Coefficients of Variation & $10.26 \%$ & $10.26 \%$ & $6.999 \%$ & $0.462 \%$ \\
\hline
\end{tabular}




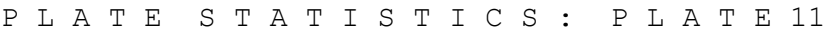

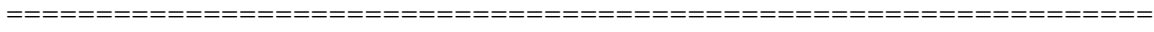

Crack Initiation at Insert

\begin{tabular}{|c|c|c|c|c|c|c|c|}
\hline $\begin{array}{l}\mathrm{a} 0 \\
(\mathrm{in})\end{array}$ & $\begin{array}{l}\text { disp } \\
\text { (in) }\end{array}$ & $\begin{array}{c}P \\
(1 \mathrm{bf})\end{array}$ & $\begin{array}{c}\text { GIC } \\
(p s i-i n)\end{array}$ & $\begin{array}{c}\mathrm{MBT} \\
(\text { ) }\end{array}$ & $\begin{array}{l}\mathrm{CC} \\
()\end{array}$ & $\begin{array}{c}\mathrm{MCC} \\
()\end{array}$ & $\begin{array}{c}\text { EQS } \\
(\text { ) }\end{array}$ \\
\hline \multicolumn{8}{|c|}{ Mean Values----------------------------------------------------- } \\
\hline 2.047 & 0.409 & 12.58 & NL & 3.693 & 3.552 & 3.532 & 2.827 \\
\hline 2.047 & 0.434 & 13.21 & vis & 4.122 & 3.962 & 3.924 & 3.11 \\
\hline 2.047 & $\mathrm{NaN}$ & $\mathrm{NaN}$ & $5 \%$ & $\mathrm{NaN}$ & $\mathrm{NaN}$ & $\mathrm{NaN}$ & \\
\hline Standard & \multicolumn{7}{|c|}{ Deviations--------------------------------------------- } \\
\hline $1.80 e-05$ & 0.010 & 0.461 & $\mathrm{NL}$ & 0.206 & 0.291 & 0.332 & 0.192 \\
\hline $1.80 e-05$ & 0.010 & 0.455 & vis & 0.203 & 0.287 & 0.325 & 0.174 \\
\hline $1.80 e-05$ & 0.010 & $\mathrm{NaN}$ & $5 \%$ & $\mathrm{NaN}$ & $\mathrm{NaN}$ & $\mathrm{NaN}$ & $\mathrm{NaN}$ \\
\hline \multicolumn{8}{|c|}{ Coefficients of Variation $\left(\frac{\circ}{\circ}\right)-----------------------------------1$} \\
\hline $8.79 e-04$ & 2.445 & 3.670 & NL & 5.571 & 8.195 & & 6.801 \\
\hline $9 e-04$ & 2.329 & 3.446 & vis & 4.913 & 7.236 & 8.271 & 5.579 \\
\hline $79 e-04$ & NaN & $\mathrm{NaN}$ & $5 \%$ & $\mathrm{NaN}$ & $\mathrm{NaN}$ & $\mathrm{NaN}$ & \\
\hline
\end{tabular}

Crack Initiation at Precrack

\begin{tabular}{|c|c|c|c|c|c|c|c|}
\hline $\begin{array}{l}\operatorname{ap} \\
(i n)\end{array}$ & $\begin{array}{l}\text { disp } \\
(i n)\end{array}$ & $\begin{array}{c}\mathrm{P} \\
(1 \mathrm{bf})\end{array}$ & $\begin{array}{c}\text { GIC } \\
(p s i-i n)\end{array}$ & $\begin{array}{c}\mathrm{MBT} \\
()\end{array}$ & $\begin{array}{l}\mathrm{CC} \\
()\end{array}$ & $\begin{array}{c}\mathrm{MCC} \\
()\end{array}$ & $\begin{array}{c}\text { EQS } \\
()\end{array}$ \\
\hline \multicolumn{8}{|c|}{ 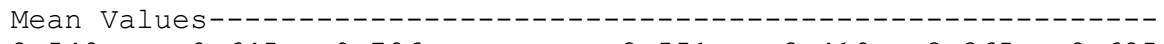 } \\
\hline 2.549 & 0.645 & 9.796 & $\mathrm{NL}$ & 3.551 & 3.419 & 3.365 & 2.625 \\
\hline 2.549 & 0.686 & 10.39 & vis & 4.044 & 3.886 & 3.648 & \\
\hline 2.549 & NaN & $\mathrm{NaN}$ & $5 \%$ & NaN & NaN & NaN & $\mathrm{NaN}$ \\
\hline \multicolumn{8}{|c|}{ Standard Deviations--------------------------------------------- } \\
\hline $2.64 e-01$ & 0.155 & 0.934 & NL & 0.092 & 0.092 & 0.117 & \\
\hline $2.64 e-01$ & 0.159 & 0.857 & vis & 0.178 & 0.244 & 0.231 & \\
\hline $2.64 e-01$ & 0.155 & $\mathrm{NaN}$ & $5 \%$ & $\mathrm{NaN}$ & $\mathrm{NaN}$ & $\mathrm{NaN}$ & \\
\hline \multicolumn{8}{|c|}{ Coefficients of Variation (응)---------------------------------- } \\
\hline $1.03 e+01$ & 23.96 & 9.538 & NL & 2.578 & 2.695 & 3.469 & \\
\hline $1.03 e+01$ & 23.13 & 8.251 & vis & 4.411 & 6.267 & 6.334 & \\
\hline $1.03 e+01$ & NaN & $\mathrm{NaN}$ & $5 \%$ & $\mathrm{NaN}$ & $\mathrm{NaN}$ & $\mathrm{NaN}$ & \\
\hline
\end{tabular}

Crack Propagation Values

\begin{tabular}{|c|c|c|c|c|}
\hline & GIC-MBT & $\mathrm{GIC}-\mathrm{CC}$ & $\mathrm{GIC}-\mathrm{MCC}$ & GIC-EQS \\
\hline Mean Values & 4.472 & 4.295 & 4.290 & 3.479 \\
\hline Standard Deviations & 0.464 & 0.443 & 0.420 & 0.314 \\
\hline Coefficients of Variation & $10.30 \%$ & $10.30 \%$ & $9.772 \%$ & $8.888 \%$ \\
\hline
\end{tabular}

Slopes and Intercepts

$\begin{array}{lccc} & \text { Delta } & \text { n } & \text { A1 } \\ -----------1 & & 48.36 \\ \text { Mean Values } & -3.095 e-017 & 2.882 & 1.840 \\ \text { Standard Deviations } & 7.58 e-017 & 0.084 & 3.802 \% \\ \text { Coefficients of Variation } & -244.9 \% & 2.912 \% & \end{array}$

